\title{
Portfolio Strategies with Classical and Alternative Benchmarks
}

\author{
Dissertation
}

zur Erlangung des Doktorgrades

der Wirtschaftswissenschaftlichen Fakultät

der Georg-August-Universität Göttingen

vorgelegt von

Laura-Chloé Kuntz, M.Sc.

aus Koblenz

Göttingen, 2018 


\section{Thesis Committee:}

Prof. Dr. Olaf Korn

Prof. Dr. Jörg-Markus Hitz

Prof. Dr. Kilian Bizer 


\section{Contents}

List of Tables $\quad$ iii

List of Figures $\quad$ v

1 Introduction 1

2 Low-beta Strategies $\quad 12$

2.1 Introduction . . . . . . . . . . . . . . . . . . . . . 13

2.2 Characterizing Low-beta Strategies . . . . . . . . . . . . . . . . . 16

2.2.1 Definition of Low-beta Strategies . . . . . . . . . . . . . . 16

2.2.2 Basic Low-beta Strategies . . . . . . . . . . . . . . . 18

2.2.3 Additional Design Elements of Low-beta Strategies . . . . . . . . 20

2.3 Data and Design of the Empirical Study . . . . . . . . . . . . . . 22

2.4 Empirical Results . . . . . . . . . . . . . . . . . . . 25

2.4.1 Return and Risk Characteristics for the Base Case . . . . . . . . . . 25

2.4.2 Effects of Additional Design Elements . . . . . . . . . . . . . 31

2.4 .3 Using a Smaller Investible Universe . . . . . . . . . . . . . . . 50

2.5 Conclusion . . . . . . . . . . . . . . . . . . . 53

3 Markowitz with Regret $\quad 55$

3.1 Introduction . . . . . . . . . . . . . . . . . . 56

3.2 The Portfolio Problem . . . . . . . . . . . . . . . . . . . . . 59

3.3 Optimal Portfolios . . . . . . . . . . . . . . . . . . 67

3.3 .1 Two-asset Case . . . . . . . . . . . . . . . . . 67

3.3.2 Multi-asset Case . . . . . . . . . . . . . . 76

3.4 Regret Effects for Real Portfolios . . . . . . . . . . . . . . . . . . . 82

3.4 .1 Data and Empirical Setup . . . . . . . . . . . . . . . . 82

3.4.2 Results......................... . . 84 
3.5 Conclusion . . . . . . . . . . . . . . . . . . . . 93

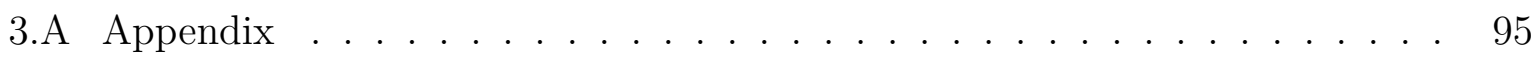

3.A.1 Optimal Portfolio Weights . . . . . . . . . . . . . . 95

3.A.2 Impact of Expected Return Changes on Optimal Portfolio Weights 96

4 Beta Dispersion and Market Timing $\quad 98$

4.1 Introduction . . . . . . . . . . . . . . . . . . . . . . . . . . 99

4.2 Systematic Risk and Market Return . . . . . . . . . . . . . . . . . 103

4.2.1 Stochastic Beta and Market Timing . . . . . . . . . . . . . . 103

4.2.2 Beta Dispersion as a Measure of Market Vulnerability . . . . . . . . 105

4.3 Data and Methodology . . . . . . . . . . . . . . . 108

4.4 Empirical Implementation and Results . . . . . . . . . . . . . . . . 109

4.4.1 Empirical Decomposition of Returns . . . . . . . . . . . . . . . 109

4.4.2 Empirical Description of the Beta Dispersion . . . . . . . . . . . . 111

4.4.3 Predicting Market Returns - In-sample Evaluation . . . . . . . . . 115

4.4.4 Predicting Market Returns - Out-of-sample Evaluation . . . . . . . 127

4.4.5 Market Timing Strategies Using Beta Dispersion . . . . . . . . . . . 132

4.5 Conclusion . . . . . . . . . . . . . . . . . . . . . . . 142

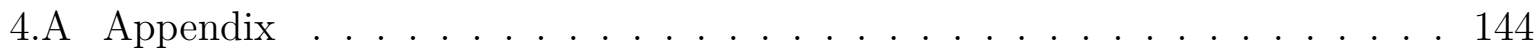

4.A.1 Calculated Variables . . . . . . . . . . . . . . . . . . 144

4.A.2 Linear Predictive Regressions with $B D_{V W}$ and $Q B D_{10 \%} \ldots \ldots$

4.A.3 Distributional Regressions . . . . . . . . . . . . . . . 147

$\begin{array}{llr}5 & \text { Conclusion } & 149\end{array}$

$\begin{array}{lr}\text { Bibliography } & 154\end{array}$

$\begin{array}{lr}\text { Declaration } & 167\end{array}$ 


\section{List of Tables}

2.1 \$-Amount Invested in Various Instruments per Low-beta Strategy . . . . . 19

2.2 Overview of Additional Design Elements of Low-beta Strategies . . . . . . 24

2.3 Base Case - Return and Risk . . . . . . . . . . . . . . . . . . . 28

2.4 Estimation Method: Frazzini/Pedersen - Return and Risk . . . . . . . . 33

2.5 Estimation Method: Dimson - Return and Risk . . . . . . . . . . 35

2.6 Estimation Period: 1 Month - Return and Risk . . . . . . . . . . . . 37

2.7 Estimation Period: 12 Months - Return and Risk . . . . . . . . . . . . . . 39

2.8 Portfolio Coverage: 2\% (30 Stocks) - Return and Risk . . . . . . . . . 41

2.9 Portfolio Coverage: 20\% (300 Stocks) - Return and Risk . . . . . . . . . . 43

2.10 Weighting within the Portfolios: Equal Weighting - Return and Risk . . . 45

2.11 Weighting within the Portfolios: Value Weighting - Return and Risk . . . 47

2.12 Rebalancing: Monthly - Return and Risk . . . . . . . . . . . . . . . 49

2.13 Investible Universe: Smaller Universe - Return and Risk . . . . . . . . . . 51

3.1 Description of the Investible Universes . . . . . . . . . . . . . . . 83

3.2 Results for the Stock-Bond Universe . . . . . . . . . . . . . . . . . . 86

3.3 Results for the Country Universe . . . . . . . . . . . . . . . . 88

3.4 Results for the Investment Style Universe . . . . . . . . . . . . . . . . . . 90

3.5 Results for the Continent Universe . . . . . . . . . . . . . . . . . . 92

4.1 Decomposition of Returns: Market Timing Component . . . . . . . . . . . 110

4.2 Linear Predictive Regressions with $B D_{E W} \ldots \ldots \ldots \ldots$. . . . . . 117

4.3 Predictive Regressions with Non-overlapping Periods . . . . . . . . . . . 119

4.4 Predictive Regressions with Dummy for Negative Market Returns . . . . . 121

4.5 Predictive Regressions with Additional Explanatory Variables . . . . . . . 123

4.6 Results of the Out-of-sample $R_{O S}^{2}$ for $B D_{E W} \ldots \ldots \ldots$. . . . . . . . 129

4.7 Performance of Market Timing Strategies . . . . . . . . . . . . . . . 139 
4.A1 Linear Predictive Regressions with $B D_{V W} \ldots \ldots \ldots$. . . . . . . . . . 145

4.A2 Linear Predictive Regressions with $Q B D_{10 \%} \ldots \ldots \ldots$ 


\section{List of Figures}

1.1 Elements of Benchmark Specification . . . . . . . . . . . . 4

2.1 Betas of the Low-beta Portfolio and the High-beta Portfolio . . . . . . . 25

2.2 Increase in Total Wealth Resulting from Low-beta Strategies . . . . . . . . 26

2.3 Beta of the Low-beta Portfolio for Different Estimation Methods . . . . . . 31

3.1 Effects of a Shift in Expected Return . . . . . . . . . . . . . . 69

3.2 Effects of a Shift in Standard Deviation . . . . . . . . . . . . . . 71

3.3 Effects of a Shift in Standard Deviation with Different Expected Returns . 73

3.4 Effects of a Shift in Skewness _ . . . . . . . . . . . . . . . 75

3.5 Effects of a Shift in Standard Deviation with Multiple Assets . . . . . . . . 79

3.6 Effects of a Shift in Correlation . . . . . . . . . . . . . . . 81

4.1 Time Series of Beta Dispersion and S\&P 500 Index Level . . . . . . . . . . 112

4.2 Beta Dispersion and the Concentration of Stocks in One Sector . . . . . . 114

4.3 Cumulative Sum of Differences in the Squared Forecast Errors . . . . . . . 131

4.4 Weights of Market Timing Strategy . . . . . . . . . . . . . . 135

4.5 Increase in Total Wealth Resulting from Timing Strategies . . . . . . . . . 137 


\section{Introduction}

Portfolio strategies have the objective to add value for the investor. The number of design possibilities of a portfolio is nearly unlimited, so a reasonable portfolio strategy should be based on an economic idea to determine in which assets to invest. This makes the performance of the portfolio assessable and helps to attribute the added value to expertise or luck. In general, portfolio strategies can be divided into passively and actively managed strategies. Passive strategies emulate broad asset indexes with no or only minimal adjustments in, for example, the weights of the assets. Active strategies, in contrast, try to outperform broad indexes by selecting specific assets or timing the investment. Commonly applied approaches to set up such portfolio strategies are, for instance, based on exploiting market anomalies. As essential elements of active portfolio management, designing and implementing investment strategies represent an already large and still constantly growing business sector. Nevertheless, there is still the need for further research to improve the understanding of the performance generation of portfolio strategies and influences on it. Moreover, further contributions can be made by advancing the design and implementation of strategies with innovative and newly developed methods.

To invest reasonably and to evaluate a portfolio strategy properly, a reference measure, or a benchmark, is needed. A benchmark can be understood as a reference portfolio that depicts all investment opportunities as well as the risk perception of the investor. The precise composition of such a benchmark is usually based on financial models, which imply a distinctive risk conception. A portfolio strategy is implemented within the investment opportunities comprised in the benchmark. Consequently, the role of the benchmark in finance is twofold. On the one hand, the benchmark is needed to determine a portfolio strategy, since the benchmark combines all investible assets. On the other hand, the benchmark is needed to evaluate the performance of the portfolio strategies afterwards in order to distinguish between compensation for taken risk and actual outperformance. For both, the benchmark has to reflect the investible universe and risk conception properly and, 
in addition, should match the chosen portfolio strategy to be able to serve as a reasonable reference measure and to evaluate the portfolio performance accurately. Benchmarks are needed in research and portfolio management alike; without benchmarks no reasonable development or evaluation of portfolio strategies is possible. Designing portfolio strategies and analyzing their performance is explicitly studied in literature, but the specification of benchmarks is usually not at the center of attention. Although the importance of a benchmark is undisputed, a systematic classification and impact analysis is still missing. It is in question whether different specifications of a benchmark have an influence on the exact structure of the chosen portfolio strategy and its performance. Moreover, an analysis of the implications regarding the inherent risk conception in benchmarks is often neglected. All in all, there is undeniably the need for research to shed light on the composition and use of benchmarks and stress the importance of a reasonable specification.

This dissertation addresses different key elements in portfolio management. It intends to improve and analyze influences on portfolio strategies and their performance. Likewise, it aims at the systematization and extension of benchmark specifications as well as their effect on portfolio strategies. Each chapter focuses on a different aspect of developing and implementing portfolio strategies. The dissertation seeks to contribute to the advancement of portfolio strategies by making the performance generating process and influences on it more comprehensible and transparent. In doing so, it attempts to strengthen the awareness of the impact of the exact design of portfolio strategies and benchmarks on the resulting portfolio and its performance.

As with the objective, the contribution of this thesis to portfolio strategies and benchmarks is multifaceted. Each chapter contributes on a specific issue. The following comprehensive systematization of benchmark specifications highlights the importance and complexity of an appropriate benchmark choice, demonstrates how diverse benchmark specifications can be and reveals the relation between benchmark and underlying risk conception. In Chapter 2, the first study that systematically analyzes the effect of numerous design varieties on the risk and return characteristics of portfolio strategies which are based on the same economic idea is presented. Thereby, major implications of specific portfolio choices on the risk and return characteristics are made clear. The importance and feasibility of the 
use of alternative risk conceptions is explored in Chapter 3, which make it possible to better tailor portfolio strategies to the investor needs. It is also shown that commonly applied benchmarks can be extended to more closely conform with reality. In addition, Chapter 4 develops an innovative method to design portfolio timing strategies based on recent statistical modeling techniques and introduces a new measure of market vulnerability that can be also of interest for research about financial stability and systemic risk.

The key findings of this dissertation can be summarized as follows: The benchmark specification, especially in terms of the investible universe and the inherent risk conception, has substantial influence on the explicit design and performance of portfolio strategies. In general, the specification of the benchmark and design of portfolio strategies should be carefully considered and the implementation should be well thought out. Alternative risk conceptions, such as regret risk, can be applied to portfolio selection and lead to clearly different portfolio compositions. Moreover, timing strategies can be improved by choosing a careful investment approach on the basis of distributional regressions. All empirical work of this thesis has in common that it pursues different ideas to set up portfolio strategies while explicitly addressing the benchmark specification used for the implementation and evaluation of said strategies.

Within this introduction, a comprehensive systematization for benchmark specification is derived for the first time. This is followed by a more detailed summary of the contributions and the key findings of each chapter.

\section{Conceptual Framework of Benchmark Design}

To provide a basis for the chapters of this thesis and illustrate as well as classify different benchmark specifications, a general systematization of benchmarks is developed. A comprehensive benchmark definition consists of different elements that have to be specified. ${ }^{1}$ Commonly, the concretization of these elements happens in passing when setting up port-

\footnotetext{
${ }^{1}$ In general, other approaches to systematize benchmarks are conceivable, but the here introduced scheme covers commonly applied benchmarks in finance. A different approach, for example, is a peer benchmark (Dor et al. 2008; Hunter et al. 2014): The return of a portfolio is evaluated in relation to the return of a group of portfolio, for instance investment funds, that implement comparable strategies. This peer group is regularly set by the asset manager and coincides with the group of funds the manager competes with.
} 
folio strategies and without deeper considerations of the consequences on the specification and performance of the portfolio strategy.

Figure 1.1 summarizes the elements of a comprehensive benchmark specification. To determine a benchmark, a definition of the investible universe is needed first. Within this the further dimensions, portfolio strategy and risk conception, are set to result in a suitable benchmark.

\section{Figure 1.1: Elements of Benchmark Specification}

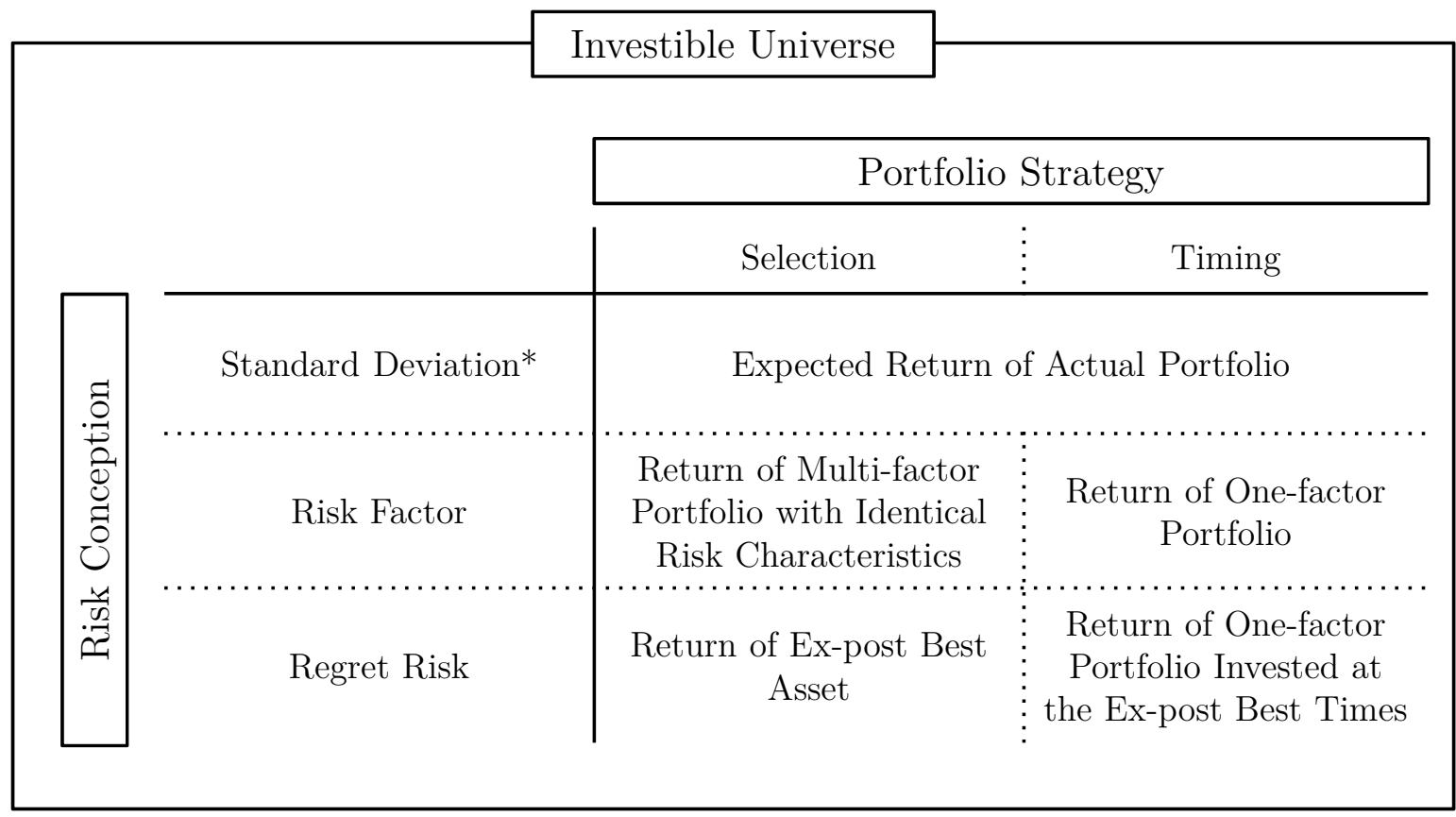

* The risk conception standard deviation can be exchanged by other risk measures, for example downside risk or expected shortfall. Nevertheless, the appropriate benchmark would still be the expected return of the actual portfolio.

Note: Figure 1.1 displays the elements of a comprehensive benchmark specification. The superordinate element is the investible universe. Within this the portfolio strategy consisting of two elements and the risk conception consisting of three elements have to be determined to generate a reasonable benchmark.

The investible universe comprises all assets in which the investor can invest. Within this framework, the investor sets up her portfolio strategy. The exact specification can have a major impact on the portfolio strategies the investor is able to implement as well as on their performances. Especially, when the portfolio strategy is based on exploiting a market anomaly, the restriction of the investment opportunities can affect the non-existence of such systematically mispriced assets. It can be shown that restricting the investible universe 
can lead to entirely different performances of otherwise equal portfolio strategies regarding the risk characteristics of the strategies as well as the outperformance of the benchmark (Korn and Kuntz $\left.(2018)^{2}\right)$.

Within the investible universe, the first dimension of the benchmark is defined by the portfolio strategy. On an efficient market in equilibrium the investor has no possibility to earn more than the risk-appropriate return as all assets are priced fairly according to their intrinsic risk. Loosening the assumption of correctly priced assets gives the investor the opportunity to earn returns without adding risk. Such portfolio strategies, which are actively managed, can be classified into two approaches: selection and timing (Sharpe 1975). Selection refers to picking single assets and generating a portfolio that is supposed to outperform the benchmark. Within this approach the investor tries to identify mispriced assets and expects to earn higher returns without additional risk. ${ }^{3}$ In contrast, when a timing approach is chosen, the investor only decides when to invest in an asset. Likewise, timing can be done for portfolios, where broad factor portfolios ${ }^{4}$, such as the market, are commonly used. This approach tries to be invested in the asset or portfolio during upward trends and not to be invested during downward trends. As such, timing tries to reduce the volatility and increase the overall return of a portfolio strategy by excluding negative returns. Therefore, the investor needs an economically reliable idea of how to distinguish between upcoming up- and downturns of the asset or portfolio. The economic applicability of such an idea should be comprehensively studied beforehand (e.g. see Kuntz $\left.(2018)^{5}\right)$.

The second dimension of benchmark design is the risk conception. Predefining a specific risk conception leads to an unambiguous benchmark specification. Vice versa, determining a benchmark implies a specific underlying risk conception. The most commonly applied risk conceptions in finance are the standard deviation of returns and theoretically and empirically derived risk factors. Regret risk is a newly introduced alternative that enlarges

\footnotetext{
${ }^{2}$ This paper is included as Chapter 2 of this thesis.

${ }^{3}$ Identifying mispriced stocks implies that one knows the fair price of an asset. Usually this is derived from a financial model which leads to the joint hypothesis problem (Fama 1970, 1991): Mispricing can be due to an actual mispricing or to a misspecification of the model ignoring certain sources of risk. Consequently, depending on the underlying model, a selection strategy's alleged outperformance may be due to the anomaly or to compensation for taken risk.

${ }^{4}$ Factor portfolios are portfolios, that depict a risk factor as sources of potential risk, for example the market. The idea of risk factors will be described in more detail below.

${ }^{5}$ This paper is included as Chapter 4 of this thesis.
} 
the set of risk conceptions and, hence, the set of benchmarks. The benchmark should be customized to the portfolio strategy as well as to the risk conception to serve as a reliable reference measure. Different benchmarks emerge from configurating the three elements which will be described below and can be seen in Figure 1.1.

The expected return of the actual portfolio as a benchmark corresponds to the standard deviation as the risk conception and is based on the seminal work of Markowitz $(1952,1987)$. He introduced the idea that the investor's objectives can be aggregated to expected return and standard deviation, so decisions can be traceably derived in this setting. The benchmark influences the portfolio strategies as it presents the investible universe; investments beyond the benchmark are not possible. Within this framework only the chosen portfolio strategy and the expectation about its return are of interest. Therefore, there is no distinction between benchmark for selection and timing. Portfolios are chosen based on their expected return and incorporated risk. The realized return of the portfolio is evaluated by comparing it to its expected value. Risk is the deviation from this benchmark, so the standard deviation. A reasonable performance measure is the well-known Sharpe ratio (Sharpe 1966, 1994), which puts the earned excess return of a portfolio into relation with incurred risk. In this setting the conception of risk can be modified and still be subsumed under the same benchmark specification. Different approaches have been studied and all have in common that the standard deviation is exchanged for another risk measure, for example downside risk or the expected shortfall (Klebaner et al. 2017; Quaranta and Zaffaroni 2008). Nevertheless, the expected return of the actual portfolio strategy still represents the benchmark for all of these risk approaches, so that they are subsumed to the standard deviation as risk conception.

The second commonly applied risk conception is summarized as risk factors. Here the benchmark is the return of a portfolio that has similar risk characteristics and whose return is known. For such a reference portfolio the underlying risk factors have to be identified as well as the returns of these factors. With the help of financial models the risk factors and the weight of each in the reference portfolio are determined. The benchmark deviates for selection and timing strategies. Regarding selection strategies, the most famous approach is the capital asset pricing model (CAPM), where the only risk factor is the 
systematic risk represented by the value-weighted market portfolio (Sharpe 1964; Lintner 1965; Mossin 1966). Subsequently, the return of an asset depends on its sensitivity towards systematic risk. The higher the risk sensitivity, the higher the expected return of the asset. Also, models based on the arbitrage pricing theory, such as the Fama and French (1993) three-factor model or its extensions (e.g. by Carhart (1997)) can be used to derive a risk factor benchmark. Here, more than one source of risk is identified and the expected return of an asset is potentially driven by all these factors. Irrespective of the exact model, the benchmark is the return of a (multi-)factor portfolio that has similar risk characteristics as the chosen portfolio strategy. A common performance measure is Jensen's alpha, which indicates risk-adjusted excess returns of the portfolio strategies (Jensen 1969) and reveals the outperformance of a strategy in relation to the benchmark. For timing strategies, the identification of the reference portfolio is remarkably easy. Generally, timing strategies invest in a one-factor portfolio, so that all the risk is covered by a reference portfolio of this risk factor. The only difference between benchmark and timing portfolio is that the former is held without further transactions, while the latter, is not held over the complete investment period.

The third, newly introduced risk conception is regret risk which has recently received increasing attention in finance. The feeling of regret and regret aversion are an emotion long studied in social science and decision making while using it as a risk conception in finance is rather novel. The benchmark corresponding to regret risk for selection strategies is the ex-post best asset that could have been chosen by the investor. In general, this can only be determined afterwards and is strictly dependent on the investible universe. Nevertheless, this risk conception can be integrated into portfolio selection by using the expected regret of an asset as decision criterion (Baule et al. $\left.(2018)^{6}\right)$. Regarding timing strategies, the benchmark is again a one-factor portfolio, equal to the previously explained risk factor benchmark for timing. This one-factor portfolio invests at the ex-post best points in time and avoids all negative returns. An applicable performance measure is the drawdown, which measures the cumulative loss since the start of the investment. The closer it is to zero, the better the strategy timed the factor investment. The benchmark is

\footnotetext{
${ }^{6}$ This paper is included as Chapter 3 of this thesis.
} 
expected to have a drawdown of zero as it should never be held, when the return of the factor portfolio is negative.

The dimension risk conception is not limited to the introduced conceptions, but commonly used benchmarks can be fitted into the introduced concepts. Nevertheless, an extension is possible at any time, but it should be ensured that the risk conception cannot be subsumed into the three above-mentioned categories. With this systematization, the reciprocal relation between benchmark and risk conception becomes apparent. In addition, the analyses of impacts and enlargements of benchmark specification on portfolio strategies should become more traceable with this scheme. Benchmarks outside the presented scheme are conceivable, for example peer benchmarks (Dor et al. 2008; Hunter et al. 2014), yet are not common. They represent an alternative way of attributing and evaluating the performance of portfolio strategies, but applying them to set up portfolio strategies is challenging, because the expected return of the benchmark is difficult to determine.

\section{Structure and Objective of the Thesis}

This dissertation aims to contribute to furthering the analysis and development of portfolio strategies as well as to contribute to considerations about classical and alternative benchmarks. As stated above, a benchmark is, on the one hand, used to derive portfolio strategies and serves, on the other hand, as a reference measure for the evaluation of the performance of these strategies. Thereby, the benchmark indicates the risk conception and sources of risk of the portfolio strategy. The findings of the dissertation are not only of interest for financial research, but are also highly relevant for the practice of asset management. The chapters of this dissertation show different facets of designing, improving and implementing portfolio strategies and benchmarks. The existence of the interrelation between benchmark specification and portfolio strategy can be demonstrated. Each chapter focuses on a different portfolio strategy and, consequently, on a different specification of the benchmark in terms of risk conception and purpose. All portfolio strategies are applied to settings as realistic as possible. Among others, liquidity issues and transaction costs are taken into account as well as restrictions to short-sales. Chapter 2 and 3 address selection strategies, whereas Chapter 4 develops and improves timing strategies. All introduced 
benchmark specifications are used to set up and evaluate the portfolio strategies. The key findings and contributions of each chapter are summarized in the following. ${ }^{7}$

\section{Chapter 2: Low-beta Strategies}

This chapter compares different approaches to implement a portfolio strategy exploiting the low-beta anomaly. The purpose of this chapter is to highlight the influence of the exact specification and different design elements on a portfolio strategy. This is the first study that investigates this impact systematically. It can be shown that the different elements have substantial impact on the performance and the risk characteristics of the resulting portfolios. The benchmark occurs in this chapter in its two functions, for forming the strategies by identifying the stock to invest in and for evaluating the resulting strategies. The influence of the investible universe is explicitly studied, as the portfolio strategies are applied in two different stock universes. The specification of the benchmark has a strong effect on the performance and the risk properties of the resulting portfolio strategies. In the smaller investible universe, the performance of the strategies is not due to outperformance because all assets are apparently priced correctly. Instead the performance is only driven by risk factors and is therefore appropriate for the risk inherent in the low-beta strategy. By analyzing the portfolio performance, it is revealed that even when exploiting the same anomaly the specific design of the portfolio strategy leads to clearly different drivers of the performances in terms of risk factors. Overweighting high-beta stocks leads to considerably different risk-adjusted excess returns and factor sensitivities than overweighting low-beta stocks. The study exhibits how important it is to not only detect and describe an anomaly, but also to devise concrete strategies based on such anomalies to show whether investors can actually profit from them. In addition, these finding emphasize the importance of a reasonable portfolio strategy and the sensible choice of design elements. These findings are important, for managers of delegated portfolios, who normally rely on stock universe restrictions.

\footnotetext{
${ }^{7}$ Each of the three chapters is based on a standalone empirical study.
} 


\section{Chapter 3: Markowitz with Regret}

The chapter demonstrates the successful integration of regret risk as an alternative risk conception in the portfolio selection following Markowitz $(1952,1987)$. With regret risk, the appropriate benchmark is the ex-post best asset the investor could have chosen. This study is the first that integrates regret risk and thereby extends the common benchmark specifications. It is an innovative and novel approach to include two separate investor needs (final wealth and avoidance of regret) into the portfolio optimization. Considering risk and regret risk in the portfolio composition leads to different implications for the resulting portfolio than usually supposed. The integration of regret risk should lead to more suitable portfolio strategies for investors because of the alternative and additional risk conception. This approach clearly deviates from those mentioned earlier, because another risk, beside the standard deviation, is considered instead of only exchanging one measure of risk for another. It is shown that the determination of asset weights and implementation as portfolio strategies with regret risk is as easy as in the usual framework. Regret-averse investors weight assets clearly differently than risk-averse investors. Considering regret risk can lead to more or less equal-weighted portfolios depending on the characteristics of the investible universe. A simulation as well as the empirical study show that the expected return and the skewness of the return distribution influence the preferability of an asset. Further results show that a portfolio optimized for a regret-averse investor leads to less regret compared to a conventionally designed portfolio. ${ }^{8}$ The findings are highly relevant for further research as this new approach can be extended to advanced portfolio optimization with higher moments as well as be translated onto other risk conceptions. In addition, the findings demonstrate the opportunity for investors to better customize their portfolio to their needs.

\section{Chapter 4: Beta Dispersion and Market Timing}

This chapter develops a portfolio strategy based on market timing. The innovation lies in the translation of the timing indicator into a probability for a positive future market return with the help of distributional regressions. This timing indicator, the beta dis-

\footnotetext{
${ }^{8}$ These results are not displayed in the chapter, but are available upon request.
} 
persion, is based on the conditional CAPM, serves as a measure of market vulnerability and contributes in this respect to systemic risk measures and financial stability research. Before being used to set up market timing strategies, this measure is extensively studied with a comprehensive in-sample and out-of-sample analysis of the predictive relationship of beta dispersion and market return. The vulnerability measure adds to the predictive accuracy of market return prediction and can thusly be used to construct promising timing strategies. These strategies enable the investor to avoid being invested in the market during in downturns. The evaluation of the strategies is conducted with appropriate benchmarks for all three different risk conceptions. It can be demonstrated that the risk is substantially reduced - no matter the benchmark. The novel approach of implementing timing strategies based on the conditional probability is promising and could be translated to other timing indicators to enhance these kind of portfolio strategies. For finance research, distributional regressions offer a new tool to model dependent distributions of variables with distinct advantages. Furthermore, the development of the vulnerability measure is of interest for supervisors as it captures a not yet considered dimension of market stability.

Chapter 5 concludes the thesis with a summary of the key findings, major contributions and outlook for potential further research. 


\title{
2 Low-beta Strategies
}

\author{
with Olaf Korn
}

\begin{abstract}
This paper compares several trading strategies designed to exploit the low-beta anomaly. Although the notion of buying low-beta stocks and selling high-beta stocks is natural, a choice is necessary with respect to the relative weighting of high-beta stocks and low-beta stocks in the portfolio. Our empirical results for US stocks show that this choice is very important for the risk-return characteristics of the resulting portfolios and their sensitivities to common risk factors. The weighting of stocks within the low-beta and high-beta portfolios and the chosen investible universe are essential design elements of low-beta strategies too. If smaller firms are excluded, risk-adjusted returns of low-beta strategies can even become insignificant.
\end{abstract}

\footnotetext{
Acknowledgment: Earlier versions of this article have been presented at the 2015 Australasian Finance \& Banking Conference, Sydney, the BVI-CFR Event 2015, Frankfurt, the 2016 Applied Financial Modeling Conference, Melbourne, the 2016 SGF Conference, Zürich, the 2016 Smart Beta Summit, Frankfurt, the 2016 EFMA Meeting, Basel, the 2016 FMA Meetings, Las Vegas, and the research colloquium of Georg-August-Universität Göttingen 2016. We are grateful to the discussants and participants of each seminar for their helpful comments and suggestions.
} 


\subsection{Introduction}

The observation that returns of low-beta stocks are too high and returns of high-beta stocks are too low as compared to the predictions of the standard CAPM has long been documented in the literature (Black et al. 1972; Haugen and Heins 1975; Fama and French 1992). This phenomenon, commonly referred to as the low-beta anomaly, also extends to the most recent period and is found in many different markets (Rouwenhorst 1999; Baker and Haugen 2012; Blitz et al. 2013; Frazzini and Pedersen 2014). From an investment perspective, the question arises how the low-beta anomaly could best be exploited via trading strategies, ${ }^{1}$ and from an asset pricing perspective, one would like to know how the specific construction of a corresponding risk factor affects its return, risk and co-variation with other factors. It seems intuitive to exploit the low-beta anomaly by buying low-beta stocks, selling high-beta stocks, or both, however, there are many ways to do so. What is the universe of different stocks that should be considered from the outset? How should betas be estimated? How should low-beta stocks and high-beta stocks be weighted in a portfolio? How often should these portfolios be rebalanced? The question of how these design elements of low-beta strategies affect the properties of the resulting returns, for example, alpha or sensitivities to other risk factors, is important for investors and portfolio managers alike, because the idea of exploiting the low-beta anomaly must be made concrete and requires an understanding of the implications of specific choices. This paper is the first to study the effects of all these design elements systematically.

As the starting point of our analysis, we formally define low-beta strategies as zero-cost strategies, with zero ex-ante market exposure, that are long in low-beta stocks and short in high-beta stocks. If investments in the market index and a risk-free asset are also available, this definition is fulfilled by a continuum of low-beta strategies that assign different weights to low-beta stocks and high-beta stocks. From this set of strategies, we select four basic ones covering the entire range of feasible weights. Implementation of these strategies

\footnotetext{
${ }^{1}$ Jank and Smajlbegovic (2016) show that trading based on the "betting against beta" factor proposed by Frazzini and Pedersen (2014) is common practice for institutional investors; that is, such strategies are widespread in practice. To what extent some low-beta strategies are more successful than others is an open issue, however.
} 
requires specification of several additional design elements. We consider the choice of investible universe, the method of estimating beta, the length of the time period used for beta estimation, the percentage of stocks included in the low-beta and high-beta portfolios, the weighting of individual stocks within these portfolios, and how often the portfolios are rebalanced.

Our empirical study focuses on the US stock market and shows that all design elements of low-beta strategies that we consider have an important impact on the return characteristics of the strategies. The specific design of low-beta strategies matters a great deal. This general finding is the main contribution of our study. In particular, we find that low-beta strategies that overweight (buy) low-beta stocks differ substantially from strategies that overweight (sell) high-beta stocks. Further, the method of estimating betas influences the return characteristics of all strategies. Estimating betas following the approach of Frazzini and Pedersen (2014) results in clearly higher and more aligned average returns of the strategies. This also applies for beta estimation as proposed by Dimson (1979). In addition, the weighting of single stocks within the low-beta and high-beta portfolios has a large impact on average returns and sensitivities to standard risk factors. When beta weighting and equal weighting of stocks is applied, portfolios that buy low-beta stocks perform very well. Value weighting, in contrast, leads to higher average returns for portfolios that sell high-beta stocks short. Another very important issue is the choice of investible universe. Within the universe of S\&P 1500 stocks, we find strong low-beta effects, whereas within the S\&P 500 universe, these effects disappear, showing the importance of the strategic decision to select a specific universe or benchmark.

Our paper relates to different strands of literature. First, it is naturally connected to work on the low-beta anomaly. Several analyses document the anomaly for varying time periods and markets (Rouwenhorst 1999; Baker and Haugen 2012; Blitz et al. 2013; Frazzini and Pedersen 2014; Auer and Schuhmacher 2015), and several explanations for the appearance of the phenomenon and the related low-volatility anomaly have been suggested (Baker et al. 2011; Berrada and Hugonnier 2013; Dutt and Humphery-Jenner 2013; Blitz 2014; Blitz et al. 2014; Frazzini and Pedersen 2014; Jacobs 2015; Cederburg and O'Doherty 2016; Hong and Sraer 2016; Jacobs 2016; Schneider et al. 2016; Bali et al. 2017; Christoffersen 
and Simutin 2017; Jylhä et al. 2017). Our paper has a different focus, however, because we concentrate on the comparison of different strategies that try to exploit the low-beta anomaly. Most closely related to our paper is work that investigates zero-cost strategies using short positions in high-beta portfolios and long positions in low-beta portfolios. Black (1993) analyzes such a strategy, which he calls the beta factor. ${ }^{2}$ Alternative strategies with different weighting schemes for low-beta stocks and high-beta stocks are used by Frazzini and Pedersen (2014) and Li et al. (2014). However, none of these papers analyzes the effects of varying relative weights of high- and low-beta portfolios and how theses effects interact with a wide range of additional design specifications, the focus of our work.

Current work on factor investing and smart beta strategies is also related to our study. Important contributions to this literature investigate if and how the performance of valueweighted indexes can be improved via alternative weighting schemes, for example, by size, value, volatility, beta, dividend, or past return (Amenc et al. 2012; Chow et al. 2014; Hsu 2014; Jacobs and Levy 2014; Malkiel 2014; Amenc et al. 2016). Our study is in the same spirit, as we investigate how different design elements, including the weighting within a portfolio, affect performance. In particular, we show that the weighting within a low-beta portfolio, for example, equal weighting versus value weighting, can make a large difference. In addition, our results provide information on other issues discussed in the literature on smart beta and factor investing. Amenc et al. (2016) conclude that it is important to analyze the properties of different smart beta strategies individually, and one must be careful with general statements. We come to the same conclusion for low-beta strategies. Blitz et al. (2014) discuss whether a long-only approach for factor investing is more efficient than a long-short approach. Likewise, we compare strategies based on buying low-beta stocks with strategies selling high-beta stocks and document important differences. In particular, we find that a long-only approach is able to capture the main effects of the low-beta anomaly. Amenc et al. (2016) conduct a comparison of performance and risk of concentrated and diversified factor-tilted indexes for six factor tilts. In our study, focusing on the "low-beta factor", we also analyze the effect of using more concentrated or more diversified low-beta and high-beta portfolios and find an important effect on both mean

\footnotetext{
${ }^{2}$ The original idea goes back even to Black et al. (1972).
} 
return and risk. Finally, factor investing is much concerned with the identification of factor exposures and factor attribution, ${ }^{3}$ which motivates the design of our study. The low-beta strategies that we use have zero ex-ante beta by construction and therefore no ex-ante correlation with the market factor, meaning that we disentangle the low-beta premium from the market risk premium. Such a property can not be easily achieved for the low-volatility factor. ${ }^{4}$ We therefore concentrate on low-beta strategies and do not consider low-volatility strategies.

This paper proceeds as follows. Section 2.2 provides a formal definition of low-beta strategies, introduces four basic strategies, and discusses additional design elements that need to be specified before implementation. The following Section 2.3 introduces the data and design of our study. Section 2.4 presents our empirical results on the return characteristics of the specified low-beta strategies. Section 2.5 sets forth our conclusions.

\subsection{Characterizing Low-beta Strategies}

\subsubsection{Definition of Low-beta Strategies}

For our investigation on the impact of different choices a portfolio manager can make when implementing a low-beta strategy we must state precisely what a low-beta strategy is. To define such a strategy, we suggest the fulfillment of four conditions. These conditions ensure that a strategy is in line with the intuitive notion of low-beta investing and that it puts different strategies on an equal footing, to make comparison meaningful.

In our setting, investors can form portfolios from a universe of $N$ stocks. These $N$ stocks constitute the "market", and betas of individual stocks are defined in relation to this market portfolio. We assume that an investment in the market portfolio is possible, via ETFs, futures, or by buying stocks directly. Moreover, there is also a risk-free investment (and financing) available. By definition, the beta of the market portfolio equals 1 . It is

\footnotetext{
${ }^{3}$ For example, Blitz (2016) investigates the relation between the low-volatility factor and the value factor.

${ }^{4}$ Seminal papers on the low-volatility anomaly are Ang et al. (2006) and Ang et al. (2009).
} 
therefore a natural requirement for a low-beta portfolio to have a beta below 1 and for a high-beta portfolio to have a beta above 1 . Based on this notion of low-beta and high-beta portfolios, we define a low-beta strategy via the following conditions:

Condition (i): Denote the amount invested in a selected low-beta portfolio $L$ by $X_{L}$ and the amount invested in a selected high-beta portfolio $H$ by $X_{H}$. Then, a low-beta strategy requires $X_{L} \geq 0$ and $X_{H} \leq 0$, with at least one of the conditions holding as a strict inequality.

Condition (i) states that a low-beta strategy is a long-short strategy that goes long a low-beta portfolio and short a high-beta portfolio. However, as it is a goal of this paper to investigate the roles of low-beta and high-beta portfolios in low-beta strategies, we also allow for the extreme cases that take only long positions in low-beta portfolios or only short positions in high-beta portfolios.

Condition (ii): The (ex-ante) beta of a low-beta strategy is zero. Formally, this condition can be expressed as $X_{L} \beta_{L}+X_{H} \beta_{H}+X_{M}=0$, where $X_{M}$ denotes the amount invested in the market portfolio and $\beta_{L}$ and $\beta_{H}$ are the betas of the low-beta and high-beta portfolios, respectively.

The goal of low-beta strategies is to exploit the differential performance of high-beta and low-beta stocks. To concentrate on this differential, (i.e., the "betting-against-beta factor") the returns of these strategies should be isolated as far as possible from the market factor. To achieve this, at least on an ex-ante basis using estimated betas, the beta of the strategy should be zero, which is what condition (ii) states.

The next two conditions facilitate comparison between different low-beta strategies by ensuring homogeneity in specific aspects.

Condition (iii): A low-beta strategy has an initial value of zero. Formally, this condition reads $X_{L}+X_{H}+X_{M}+X_{R}=0$, where $X_{R}$ denotes the amount invested in the risk-free asset. 
Condition (iv): The sum of the absolute dollar amounts invested in the low-beta portfolio and the high-beta portfolio is the same for different low-beta strategies, that is, $\left|X_{L, i}\right|+$ $\left|X_{H, i}\right|=\left|X_{L, j}\right|+\left|X_{H, j}\right|$, where $i$ and $j$ denote different low-beta strategies.

Condition (iii) states that any low-beta strategy has the same initial amount invested, with zero as a natural choice. ${ }^{5}$ Condition (iv) states that all low-beta strategies generate the same amount of total (dollar) trading volume (either long or short) in the low-beta and high-beta portfolios. We concentrate on the trading volume in the high-beta and low-beta portfolios because these portfolios usually consist of many different stocks and trading can generate significant transaction costs. In contrast, trading in the risk-free instrument and the market is much cheaper if appropriate derivatives (interest rate futures, index futures, ETFs) are available.

\subsubsection{Basic Low-beta Strategies}

Our definition of low-beta strategies leaves substantial flexibility with respect to the portfolio weights assigned to the low-beta portfolio, the high-beta portfolio, the market index, and the risk-free instrument. For our empirical investigation, we consider a range of four basic low-beta strategies that cover natural reference points and extreme cases. In particular, they provide evidence on the performance contribution of the high-beta portfolio and the low-beta portfolio. The basic low-beta strategies are defined as follows:

Balanced ( $B L)$ : A natural starting point is a strategy that invests 1 dollar in the low-beta portfolio and is short 1 dollar in the high-beta portfolio, that is, $X_{L}=1$ and $X_{H}=-1$. It follows from condition (ii) in the previous section that $X_{M}=\beta_{H}-\beta_{L}$, and condition (iii) finally implies that $X_{R}=\beta_{L}-\beta_{H}$.

Extreme Low (EL): A first extreme case takes a long position in the low-beta portfolio but no position in the high-beta portfolio. To fulfill condition (iv), in relation to the balanced strategy, we obtain $X_{L}=2$ and $X_{H}=0$. From conditions (ii) and (iii), the investments in the index and the risk-free instrument become $X_{M}=-2 \beta_{L}$ and $X_{R}=2 \beta_{L}-2$, respectively.

\footnotetext{
${ }^{5}$ Of course, other choices for the initial value, like a value of 1 dollar, could be considered. However, given that a strategy already fulfills conditions (i), (ii), and (iv), we could simply change the position in the risk-free asset to fulfill such a modified condition (iii).
} 
Extreme High (EH): The extreme high strategy is the mirror image of the extreme low strategy. It takes a short position in the high-beta portfolio and no position in the low-beta portfolio, that is, $X_{H}=-2$ and $X_{L}=0$. From conditions (ii) and (iii), the investments in the index and the risk-free instrument become $X_{M}=2 \beta_{H}$ and $X_{R}=-2 \beta_{H}+2$, respectively. No Market Investment (NM): The weighting used in the fourth strategy is based on the idea described in Frazzini and Pedersen (2014) that no investment in the index is required, that is, $X_{M}=0$. The fulfillment of conditions (i), (ii), and (iv) then implies that $X_{H}=-2 \beta_{L} /\left(\beta_{H}+\beta_{L}\right)$ and $X_{L}=2 \beta_{H} /\left(\beta_{H}+\beta_{L}\right)$. From condition (iii), we finally obtain $X_{R}=2\left(\beta_{L}-\beta_{H}\right) /\left(\beta_{H}+\beta_{L}\right)$. For this strategy, the amounts invested in the low-beta and high-beta portfolios therefore depend on the magnitudes of the corresponding betas.

Table 2.1 provides an overview of the dollar amounts invested in various instruments according to the four basic strategies. Clearly, the four strategies give different weights to high-beta and low-beta portfolios. The low-beta portfolio is most important, in terms of absolute weights, for the EL strategy, followed by NM, BL, and EH. Also note that one has to take a long position in the market for both the EH and BL strategies, whereas the NM strategy uses a zero position in the market by construction and the EL strategy takes a short market position. All four low-beta strategies require risk-free borrowing.

Table 2.1: \$-Amount Invested in Various Instruments per Low-beta Strategy

\begin{tabular}{|c|c|c|c|c|c|}
\hline & & \multicolumn{4}{|c|}{ Strategy } \\
\hline & & Extreme Low & No Market & Balanced & Extreme High \\
\hline \multirow{4}{*}{ 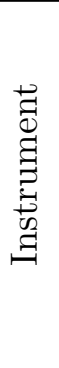 } & Low-beta & 2 & $\frac{2 \beta_{H}}{\beta_{H}+\beta_{L}}$ & 1 & - \\
\hline & High-beta & - & $\frac{-2 \beta_{L}}{\beta_{H}+\beta_{L}}$ & -1 & -2 \\
\hline & Market & $-2 \beta_{L}$ & - & $\beta_{H}-\beta_{L}$ & $2 \beta_{H}$ \\
\hline & Risk-free & $2 \beta_{L}-2$ & $\frac{2\left(\beta_{L}-\beta_{H}\right)}{\beta_{H}+\beta_{L}}$ & $\beta_{L}-\beta_{H}$ & $-2 \beta_{H}+2$ \\
\hline
\end{tabular}

Note: Table 2.1 shows the dollar amounts invested in the low-beta portfolio (Low-beta), the high-beta portfolio (High-beta), the market index (Market), and the risk-free asset (Risk-free) for all four basic low-beta strategies (Extreme Low, No Market Investment, Balanced, Extreme High).

Concluding our introduction of the four basic strategies, we highlight how the NM strategy is related to the other strategies. For the NM strategy, the investments in the low-beta 
and high-beta portfolios depend on the relation of the corresponding betas, and it is instructive to examine some extreme parameter constellations. If $\beta_{L}$ goes to zero or $\beta_{H}$ goes to infinity, the NM strategy converges to the EL strategy. ${ }^{6}$ As another extreme case, consider $\beta_{L}=\beta_{H}$. Under this parameter constellation, the NM strategy coincides with the BL strategy. We can therefore conclude that the NM strategy generally falls between the EL and BL strategies. The larger the deviation between $\beta_{L}$ and $\beta_{H}$, the more the NM strategy behaves like the EL strategy, and the smaller the deviation, the more it behaves like the BL strategy.

\subsubsection{Additional Design Elements of Low-beta Strategies}

In addition to the relative weighting of the low-beta and high-beta portfolios, choices on several other design elements affecting the actual set up of a strategy are required, for example, selection of the market, estimation methods, estimation periods, weighting schemes within the low-beta and high-beta portfolios, and the rebalancing frequency. Whereas selection of the market or investible universe is typically a strategic choice of the asset management firm or a particular client, the other choices mentioned above are usually made by the portfolio manager.

On the most general level, the market (investible universe) must be specified. In the literature, the low-beta anomaly is studied mainly for an investible universe that is as broad as possible; that is, studies for the US stock market use the CRSP universe. ${ }^{7}$ Such a choice is reasonable if the anomaly itself is the focus of the investigation. However, our focus is on low-beta strategies. In this context, it makes sense to examine smaller universes consisting of more liquid stocks and with active markets for derivatives written on the universe. The benefits are lower transaction costs and a facilitated implementation of strategies. However, the anomaly may not be as strong in the smaller universe, potentially leading to smaller benefits from exploiting it.

\footnotetext{
${ }^{6}$ If $\beta_{L}$ even becomes negative, a strict implementation of the NM strategy would imply buying the high-beta portfolio, which is counter-intuitive and leads to a violation of condition (i). In our empirical analysis, we therefore use the EL strategy as an implementation of the NM strategy whenever $\beta_{L}$ becomes negative.

${ }^{7}$ A notable exception is Auer and Schuhmacher (2015), who study the low-beta effect for the 30 stocks included in Dow Jones Industrial Average (DJIA).
} 
To build low-beta and high-beta portfolios, all stocks must be ranked by their betas. This sorting is dependent on the estimated betas, and therefore on the estimation procedure. The key elements of the estimation are the specific method and the period over which the betas are determined.

The most intuitive estimation method of beta is following the CAPM, which defines beta as the covariance of the stock return and the market return divided by the variance of the market return, both computed for the same estimation window. This method is referred to as the basic approach. Another possibility included in the analysis is based on Frazzini and Pedersen (2014). Their method stresses different characteristics of correlation and standard deviation. The correlation changes more slowly than the volatility. Therefore, this approach uses different estimation windows for the standard deviation and the correlation. The former is estimated for a shorter period and describes the short-term market environment as well as the idiosyncratic risk of the stock, whereas the correlation represents the long-term systematic behavior of the stock. In addition, betas are estimated following the approach of Dimson (1979), which accounts for problems arising from infrequent trading and eliminates most of this bias. To estimate beta, the stock returns are regressed on the preceding, synchronous and subsequent market return. The stock beta is represented by the sum of the regression coefficients.

Regarding the estimation period, betas are typically estimated from monthly data over the previous five years (60 observations), because the whole CRSP universe is used and more frequent data is not available for every included stock. ${ }^{8}$ If weekly or daily data are available, already shorter estimation windows could lead to equivalent estimates.

The coverage of the low-beta and high-beta portfolios, meaning the percentage of all stocks included in these two portfolios, must be determined next. This choice influences the betas and the diversification of low-beta and high-beta portfolios. Small coverage leads to more extreme betas and less diversified portfolios. Coverage also has an impact on trading costs, which are higher the more stocks are included in the portfolios. Usually decile portfolios are formed to exploit the low-beta anomaly.

\footnotetext{
${ }^{8}$ Of course, there are also other estimation windows used in previous studies.
} 
For the weighting of single stocks within the low-beta and high-beta portfolios, an equal weighting of each stock within the corresponding portfolio would be a first, easily implementable choice. A natural alternative is value weighting, as single stocks are value-weighted in the market portfolio according to the CAPM. A third idea would be to weight individual stocks relative to their betas. This alternative emphasizes the anomaly that should be exploited, giving more weight to stocks with very low betas in the low-beta portfolio and stocks with very high betas in the high-beta portfolio. Value weighting of portfolios is often chosen to investigate the low-beta anomaly, and equal weighting and beta weighting are rather unusual. As we know from the smart beta literature, however, special attention should be paid to the weighting scheme, as it can significantly change the risk-return trade-off of a strategy.

Commonly, monthly rebalancing of portfolios is applied in the literature. For the purpose of showing the existence of the low-beta anomaly, the rebalancing frequency is of minor concern. But from an investment perspective, it could be very important, because there is again a potential trade-off. More frequent rebalancing could be beneficial to exploit the anomaly using the most recent information; however, it would also lead to higher transaction costs.

\subsection{Data and Design of the Empirical Study}

Our empirical study uses the S\&P 1500 Index and the S\&P 500 Index as the investible universe. Concentrating on the 1500 (500) most significant US stocks has the advantage that prices are available on a daily frequency and investment strategies have relatively low transaction costs. Moreover, liquid derivatives contracts on the indexes are available, which ensures that the index investments required by our strategies are cheap and easy to implement. We use daily data for the period December 1991 to April 2016. The data source for the stock price data is Thomson Reuters Datastream. As the risk-free interest rate, we use the 1-month T-bill rate from Kenneth French's website. For performance analysis of the low-beta strategies, we additionally need the factors from the Carhart (1997) four-factor model. For evaluation of our strategies, we calculate factor returns that exactly 
match our holding periods, namely, monthly, and yearly, using the monthly data provided on Kenneth French's website.

Betas are estimated with the basic method, the Frazzini/Pedersen method and the Dimson $\operatorname{method}^{9}$. For all the stocks considered in our study, daily returns are available. Betas are therefore estimated from daily returns and corresponding market returns over rolling estimation windows of 1,3 , and 12 months, respectively. ${ }^{10}$ The correlation for the Frazzini/Pedersen approach is always estimated for a rolling window of 36 months. Beta estimates are obtained for each month in the investigation period between December 1994 and April 2015. The estimates refer to the last trading day of the particular month, which is also the day on which the strategies are set up or rebalanced. The first year of the data period is required to obtain the initial beta estimates and the last year of the data period is needed to obtain the strategies' realized returns after rebalancing for the last time. Coverage of the high-beta and low-beta portfolios is set at $2 \%, 10 \%$, or $20 \%$ of the entire market. For the S\&P 1500 Index, the number of stocks in a portfolio is therefore 30, 150, or 300, respectively. For the S\&P 500, we have either 10, 50, or 100 stocks. As described, equal weighting, value weighting, and beta weighting are worthwhile alternatives for weighting within the low-beta and high-beta portfolios, so we use all three in our following investigation. Finally, we consider monthly and yearly rebalancing for the strategies.

Our study of different low-beta strategies starts with an analysis of a base case in which we examine the performance of the four basic low-beta strategies. We then observe the influence of several specifications of the additional design elements on the performance of the strategies in comparison with the base case. We first consider the design elements usually determined by the investment manager (estimation method, estimation period, portfolio coverage, weighting within portfolios, rebalancing frequency), then examine the impact of the investible universe.

\footnotetext{
${ }^{9}$ The original beta estimation method following Dimson (1979) described in the previous section, includes the subsequent market return in the regression. As we want to highlight the implementability with realistic assumptions, we also calculated the beta using only available information (i.e., the preceding and synchronous return). Since the effect is negligible, we retain with the original Dimson approach.

${ }^{10} \mathrm{~A}$ longer estimation window of 36 months leads to very similar results as the 12 -month window. Results are therefore not reported and are available upon request.
} 
The base case is defined as follows: We use the S\&P 1500 as the investible universe, because we want to start with a universe that is closer to the one usually used in previous work in terms of the number of stocks included. Beta is estimated with the basic approach over a period of three months with daily data, so that the number of observations (about 60) is comparable with previous studies. Portfolio coverage is $10 \%$ of the investible universe, that is, 150 stocks per portfolio; this is the widely used standard approach. We beta-weight the single stocks within the low-beta and high-beta portfolios to stress exploitation of the low-beta anomaly. Finally, we use yearly rebalancing to keep transaction costs as low as possible. Table 2.2 gives an overview of the various design elements and the choices made for the base case.

Table 2.2: Overview of Additional Design Elements of Low-beta Strategies

\begin{tabular}{|c|c|c|c|}
\hline Design Element & & Considered Specifications & \\
\hline Investible Universe & S\&P 1500 & & S\&P 500 \\
\hline Estimation Method & $\beta_{i}=\frac{\underset{\rho_{R_{i}, R_{M}} \sigma_{R_{i}}}{\sigma_{R_{M}}} *}{\sigma_{R^{\prime}}} *$ & $\begin{array}{l}\text { Frazzini/Pedersen } \\
\beta_{i}=\frac{\rho_{R_{i}, R_{M}} \sigma_{R_{i}}}{\sigma_{R_{M}}} * *\end{array}$ & $\begin{array}{c}\text { Dimson } \\
\beta_{i}=\sum_{j=1}^{3} \beta_{j, i} * * *\end{array}$ \\
\hline Estimation Period & 1 month & 3 months & 12 months \\
\hline Portfolio Coverage & lowest (highest) $2 \%$ & lowest (highest) $10 \%$ & lowest (highest) $20 \%$ \\
\hline Weighting within Portfolios & beta weighting & equal weighting & value weighting \\
\hline Rebalancing Frequency & monthly & & yearly \\
\hline
\end{tabular}

Note: Table 2.2 gives an overview of all additional design elements of low-beta strategies that we consider in our empirical study. The highlighted bold specification is used as the base case. $R_{i}$ is the return of stock i, $R_{M}$ is the return of the market portfolio, $\sigma_{R_{i}}$ and $\sigma_{R_{M}}$ are the standard deviation of the stock return and the market return, respectively. Lastly, $\rho_{R_{i}, R_{M}}$ is the correlation between the stock and the market return. For the Dimson estimation $R_{M, t-1}$ is the preceding market return, $R_{M, t}$ is the synchronous market return and $R_{M, t+1}$ is the subsequent market return 


\subsection{Empirical Results}

\subsubsection{Return and Risk Characteristics for the Base Case}

Since the weights assigned to the instruments by the various strategies depend on the (estimated) ex-ante betas of the low-beta and high-beta portfolios, we begin the discussion of our results with an indication of how these betas evolve over time. Figure 2.1 shows the corresponding values for the base case. Obviously, the beta of the high-beta portfolio is much more volatile over time than the beta of the low-beta portfolio. Early in the period, the beta of the low-beta portfolio is often close to zero or even negative, meaning that the NM and EL strategies coincide.

Figure 2.1: Betas of the Low-beta Portfolio and the High-beta Portfolio

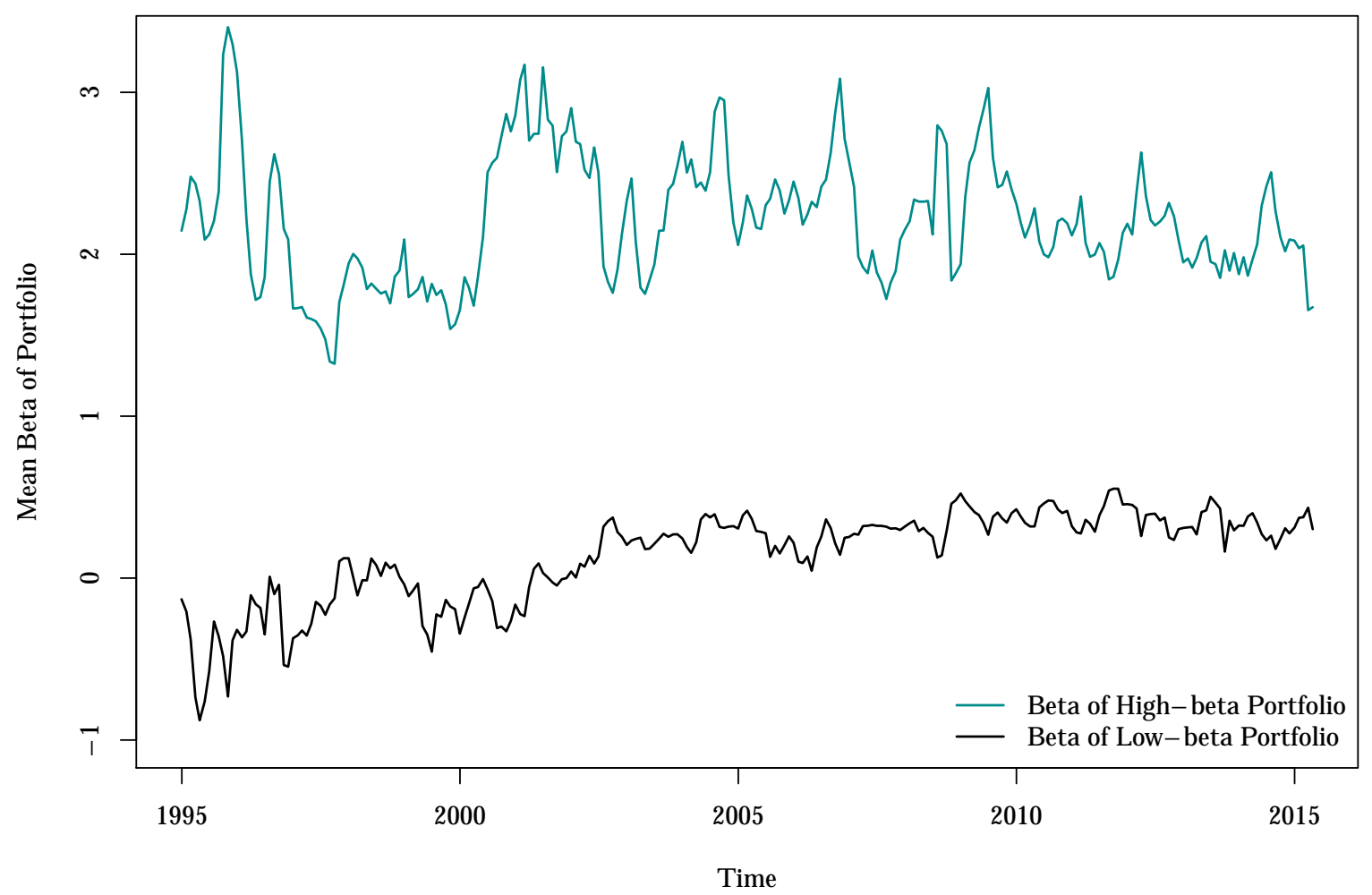

Note: Figure 2.1 shows the time series of betas of the high-beta portfolio and the low-beta portfolio in the base case scenario ( $\& \& P$ 1500, basic estimation method, beta weighting within the portfolios, $10 \%$ coverage, 3-month estimation window) over the period December 1994 to April 2015. 
Figure 2.2 shows the performance, in terms of increase in total wealth for an investor, of the four basic low-beta strategies. The increase in total wealth is measured under the base-case specification of the strategies. At the end of each month of the investigation period, an investor sets up the respective low-beta strategy, which then runs for the following 12 months. At the end of the holding period, the returns of the strategy are transferred to a money market account, which earns the risk-free rate, or, in case of a negative balance, allows for risk-free borrowing. The figure shows how the balance of this account evolves over time. For comparison, the performance of a corresponding self-financing strategy that invests in the S\&P 1500 Index and borrows at the risk-free rate is presented.

Figure 2.2: Increase in Total Wealth Resulting from Low-beta Strategies

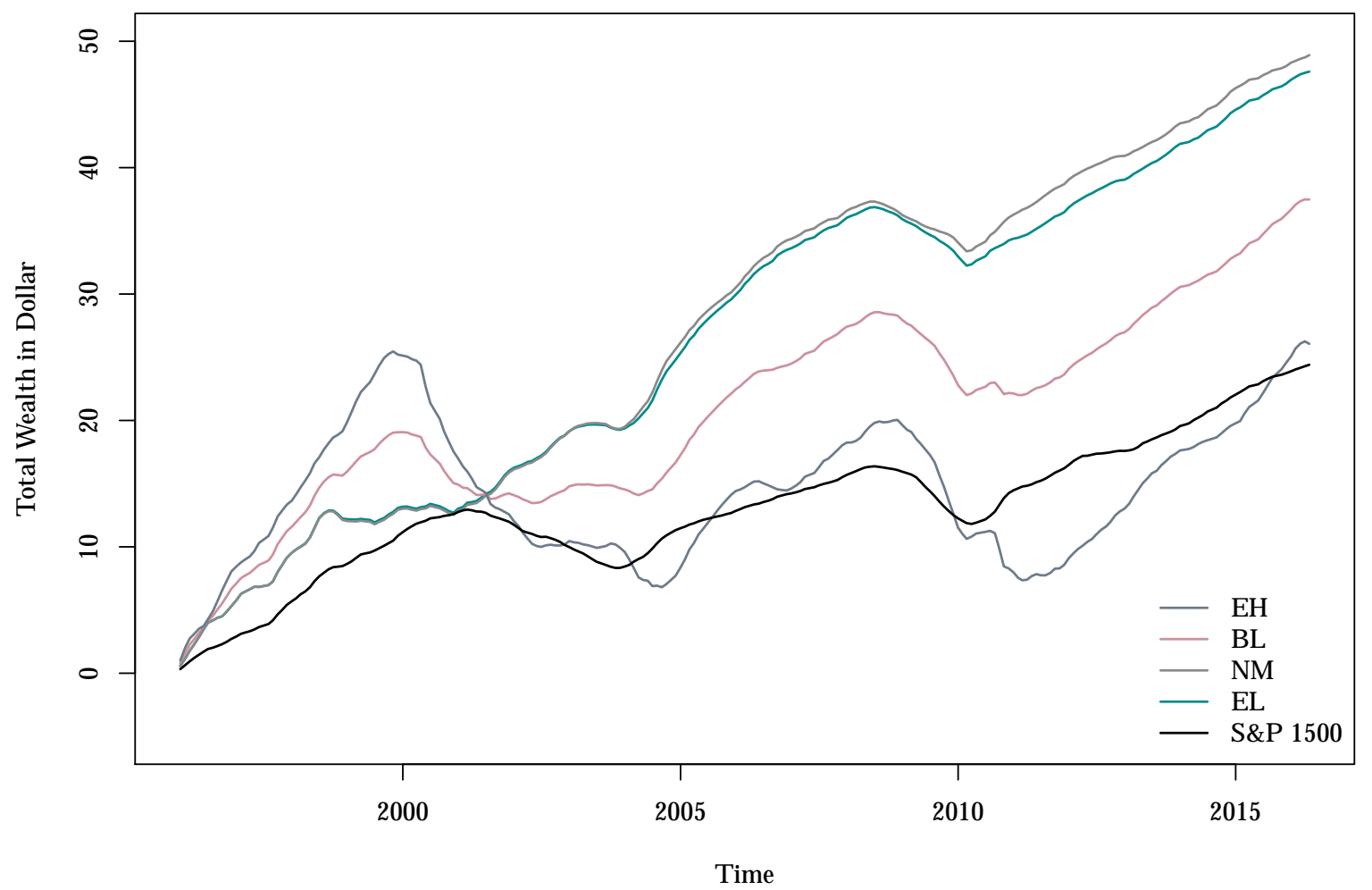

Note: Figure 2.2 shows the increase in total wealth resulting from the four low-beta strategies (extreme high (EH), balanced (BL), no market investment (NM), extreme low (EL)) and a corresponding index strategy that goes long in the S\&P 1500 Index and short in the risk-free asset. The increase in total wealth is measured under the base-case specification of the strategies ( $\& \& \mathrm{P} 1500$, basic estimation method, beta weighting within the portfolios, $10 \%$ coverage, 3-month estimation window, 12-month holding period). Investments are made every month over the investigation period, from December 1994 to April 2015, and proceeds are put into a money market account. The money market account pays the risk-free rate, or, in case of a negative balance, allows for risk-free borrowing. The figure shows the balance of the money market account over time. 
As Figure 2.2 shows, all (zero cost) low-beta strategies lead to a positive total wealth at the end of the sample period, which suggests the existence of a low-beta premium that can be exploited at least to some degree. This premium is also quantitatively quite substantial. The strategy based on the index investment essentially captures the market risk premium. Because all low-beta strategies lead to a higher total wealth at the end of the period, the low-beta premium seems to be at least as important as the market risk premium. Comparing the four basic low-beta strategies, we observe that the EL and NM strategies yield almost the same total wealth and their performance is closely related, as expected from the very small beta coefficients of the low-beta portfolio shown in Figure 2.1. Moreover, Figure 2.2 suggests that the EH strategy is more volatile than the EL and NM strategies, performing somehow better in up markets but much worse in down markets. The higher volatility is in line with the higher beta volatility of the high-beta portfolio, as compared to the low-beta portfolio. Over the entire data period, the performance of the EH strategy is clearly worse than the performance of the other three strategies.

Table 2.3 presents average yearly returns, standard deviations, Sharpe ratios, and certainty equivalent returns $(\mathrm{CEV})$ of the four basic low-beta strategies (Panel A) and the riskadjusted returns and the factor sensitivities of the strategies (Panel B). The certainty equivalent return is calculated for an investor with constant absolute risk aversion (CARA) preferences and an absolute risk aversion of $1 .{ }^{11}$ We include this measure because it depends on all moments of the return distribution, including higher order moments like skewness and kurtosis in addition to mean and standard deviation. ${ }^{12}$ Because of the zero initial investment required by our low-beta strategies, the Sharpe ratio is simply the ratio of average return and standard deviation, and the reference point for the certainty equivalent return is zero.

\footnotetext{
${ }^{11}$ Constant relative risk aversion (CRRA) preferences seem to be another natural candidate for such an analysis. However, because our low-beta strategies have discrete holding periods and contain short positions in stocks, terminal wealth cannot be guaranteed to be positive. CRRA investors would not invest in strategies that do not guarantee positive terminal wealth.

${ }^{12}$ The analysis in Schneider et al. (2016) shows that return skewness is a possible explanation for the low-beta anomaly.
} 
Table 2.3: Base Case - Return and Risk

\begin{tabular}{lcccc} 
Panel A: & \multicolumn{4}{c}{$\begin{array}{l}\text { Average Returns, Standard Deviations, Sharpe Ratios } \\
\text { and Certainty }\end{array}$} \\
& Equivalent Returns & \\
\hline Strategy & AvRet & SD & SR & CEV \\
\hline EH & 0.0861 & 0.4938 & 0.1744 & -0.0536 \\
BL & 0.1090 & 0.3049 & 0.3576 & 0.0599 \\
NM & 0.1320 & 0.2355 & 0.5617 & 0.1046 \\
EL & 0.1319 & 0.2449 & 0.5386 & 0.1025 \\
\hline
\end{tabular}

Panel B: Risk-adjusted Returns and Factor Sensitivities

\begin{tabular}{|c|c|c|c|c|c|}
\hline Strategy & $\alpha$ & $\beta_{\text {Market }}$ & $\beta_{S M B}$ & $\beta_{H M L}$ & $\beta_{M O M}$ \\
\hline $\mathrm{EH}$ & $\begin{array}{c}0.0291 \\
(0.8157)\end{array}$ & $\begin{array}{l}\mathbf{1 . 0 3 7 9} \\
(0.0008)\end{array}$ & $\begin{array}{c}0.6848 \\
(0.5112)\end{array}$ & $\begin{array}{c}0.4206 \\
(0.4678)\end{array}$ & $\begin{array}{r}-\mathbf{0 . 5 8 0 8} \\
(0.0855)\end{array}$ \\
\hline BL & $\begin{array}{c}0.0841 \\
(0.2767)\end{array}$ & $\begin{array}{l}\mathbf{0 . 4 5 4 8} \\
(0.0164)\end{array}$ & $\begin{array}{c}0.5468 \\
(0.3890)\end{array}$ & $\begin{array}{c}0.2244 \\
(0.4984)\end{array}$ & $\begin{array}{r}-\mathbf{0 . 3 8 6 2} \\
(0.0396)\end{array}$ \\
\hline NM & $\begin{array}{l}\mathbf{0 . 1 3 5 2} \\
(0.0364)\end{array}$ & $\begin{array}{c}-0.0205 \\
(0.9234)\end{array}$ & $\begin{array}{c}0.3868 \\
(0.2532)\end{array}$ & $\begin{array}{c}0.0423 \\
(0.8162)\end{array}$ & $\begin{array}{r}-\mathbf{0 . 2 2 7 0} \\
(0.0629)\end{array}$ \\
\hline EL & $\begin{array}{l}\mathbf{0 . 1 3 9 0} \\
(0.0444)\end{array}$ & $\begin{array}{c}-0.1283 \\
(0.5982)\end{array}$ & $\begin{array}{c}0.4089 \\
(0.2212)\end{array}$ & $\begin{array}{c}0.0281 \\
(0.8781)\end{array}$ & $\begin{array}{r}\mathbf{- 0 . 1 9 1 6} \\
(0.0915)\end{array}$ \\
\hline
\end{tabular}

Note: Table 2.3 shows, in Panel A, the annualized average returns (AvRet), standard deviations (SD), Sharpe ratios (SR), and certainty equivalent returns (CEV), and in Panel B, the annualized risk-adjusted returns (alphas) and factor sensitivities in a Carhart (1997) four-factor model for each of the four low-beta strategies (extreme high $(\mathrm{EH})$, balanced (BL), no market investment (NM), extreme low (EL)). The base case uses the S\&P 1500 stocks as the investible universe, the basic estimation method, an estimation period of 3 months, and a 12-month holding period. The strategies are set up at the end of each month for the period December 1994 to April 2015. Each low-beta and high-beta portfolio consists of 150 stocks, which are beta-weighted within the portfolios. The average return is the yearly return earned by each strategy, and the standard deviation is calculated from the returns for the whole investigation period. The Sharpe ratio is calculated by dividing the average return by the standard deviation, and the certainty equivalent return is calculated for an investor with CARA utility function and absolute risk aversion of 1 . The multiple linear regressions underlying the results of Panel B use four independent variables (market excess return, SMB, HML, MOM) and read $R_{t}=\alpha+\beta_{\text {Market }} \cdot\left(R_{M, t}-R_{f, t}\right)+\beta_{S M B} \cdot S M B_{t}+\beta_{H M L} \cdot H M L_{t}+\beta_{M O M} \cdot M O M_{t}+\epsilon_{t}$, where $\left(R_{M, t}-R_{f, t}\right)$ is the excess return of the market proxy at time $t$ and $S M B_{t}, H M L_{t}$ and $M O M_{t}$ are the returns of the factor-mimicking portfolios for size, value, and momentum effects, respectively. The calculations of the p-values (in parentheses) use the Newey-West estimator with 11 lags to account for the overlapping periods. Coefficients that are significant at least at a $10 \%$ level are printed in boldface. 
The average returns in Panel A show a clear pattern. When moving from the EH strategy to the strategies that give a higher (absolute) weight to the low-beta portfolio (NM, EL), the average return clearly increases. This finding shows that which weighting scheme is employed can make a difference. The standard deviations of the four strategies also show a clear pattern; that is, the more high-beta stocks are overweighted, the higher is the standard deviation. If we consider the Sharpe ratio and the certainty equivalent return as measures of investment performance, the best-performing strategies are those with a high weight in the low-beta portfolio, where it is remarkable that the NM strategy provides the best risk-return trade-off. Both measures lead to the same ranking of different strategies. Since it is an important question how the observed patterns of average returns for different low-beta strategies can be explained, we address this question in Panel B. One idea is to ask to what extent the returns of individual low-beta strategies show different sensitivities to common risk factors. To investigate this issue, we use the Carhart (1997) four-factor model that augments the Fama and French (1993) three-factor model with a momentum factor. We regress the returns of the low-beta strategies on the returns of the four-factor portfolios (market, size, value, momentum) and obtain factor loadings for each of the four factors and each of the four low-beta strategies. ${ }^{13}$

Panel B of Table 2.3 shows the alphas ${ }^{14}$ and the factor loadings for all four strategies. The observed pattern in average returns can be found again for the alphas. The more the low-beta stocks are overweighted the higher is the alpha. Moreover, the alphas of the NM and the EL strategies are statistically significant, as it is evident from the p-values below $5 \%$, whereas the EH and BL strategies yield no significant alphas. It is remarkable that the average risk-adjusted returns of the NM and EL strategies are slightly higher than the average raw return. For the EH and BL strategies, however, risk-adjusted returns are much lower. An explanation for this can be found in the market exposure, which is positive and highly significant for the EH and BL strategies. Market exposure decreases with the weight shifted to the low-beta stocks; for the NM and the EL strategies, it is negative but insignificant. A reason for the higher market exposure of the EH strategy could be a higher

\footnotetext{
${ }^{13}$ This analysis corresponds to an evaluation with an appropriate risk-factor benchmark, as introduced in Chapter 1.

${ }^{14}$ These alphas correspond to the described Jensen's alpha in Chapter 1.
} 
estimation risk for high-beta stocks, which is in line with the strong variation of betas for the high-beta portfolio shown in Figure 2.1. If beta estimates contain a lot of noise, the beta of the high-beta portfolio is likely to be upward biased, due to a misclassification of stocks in the top beta decile. Due to such a bias, the long position in the market index would be too high and would cause an overall positive market exposure. The momentum exposure is negative and significant for all four strategies, with an increasing coefficient from the EH to the EL strategies. The negative exposure of the NM and the EL strategy with respect to the momentum factor could explain why the average risk-adjusted returns of these two strategies are slightly higher than the average raw returns.

From an investment perspective, it is important to know whether the alphas of the NM and EL strategies remain statistically and economically significant under transaction costs. To analyze this issue, we follow the approach of Grundy and Martin (2001) and calculate the implicit transaction costs that would still support significant positive alphas. Assuming an investment horizon of one year, which would require no rebalancing in our base case, we use a turnover of $200 \%$, that is, all stocks are bought at the beginning of a 12-month period and sold at the end. For a 5\%-significance level, the EL and NM strategies still yield significant alphas with very substantial transaction cost of up to 190 bps. The corresponding alphas are $10.04 \%$ and $9.88 \%$, respectively. For a $10 \%$-significance level, transaction costs can be as high as 270 bps. The corresponding alphas would be $8.44 \%$ for the EL strategy and $8.28 \%$ for the NM strategy.

From the analysis of the average returns, Sharpe ratios, alphas, and factor sensitivities, it is obvious that it makes a huge difference how the low-beta anomaly is exploited. Shifting weights from high-beta stocks to low-beta stocks leads to higher average returns, lower standard deviations, statistically and economically more significant alphas, and lower (absolute) factor sensitivities with respect to all four factors of the Carhart model, making a strong point for the use of the low-beta portfolio in an investment strategy. In particular, alpha seems to be generated predominantly by the long positions of low-beta stocks. This result is also good news for investors who are reluctant to follow long-short strategies, because the low-beta premium (alpha) can be very well exploited by a strategy (EL) that does not require any short sales of high-beta stocks. 


\subsubsection{Effects of Additional Design Elements}

\subsubsection{Alternative Estimation Method}

The first variation from the base case is the estimation method of beta. Figure 2.3 shows the mean beta of the low-beta portfolio (three month estimation period, 150 stocks, beta weighting), based on the three different estimation methods for beta. ${ }^{15}$ The portfolio beta estimated with the basic and the Dimson method are similar, although the later varies much more and is generally lower. The portfolio beta based on Frazzini/Pedersen method is much more stable and always produces positive portfolio betas.

\section{Figure 2.3: Beta of the Low-beta Portfolio for Different Estimation Methods}

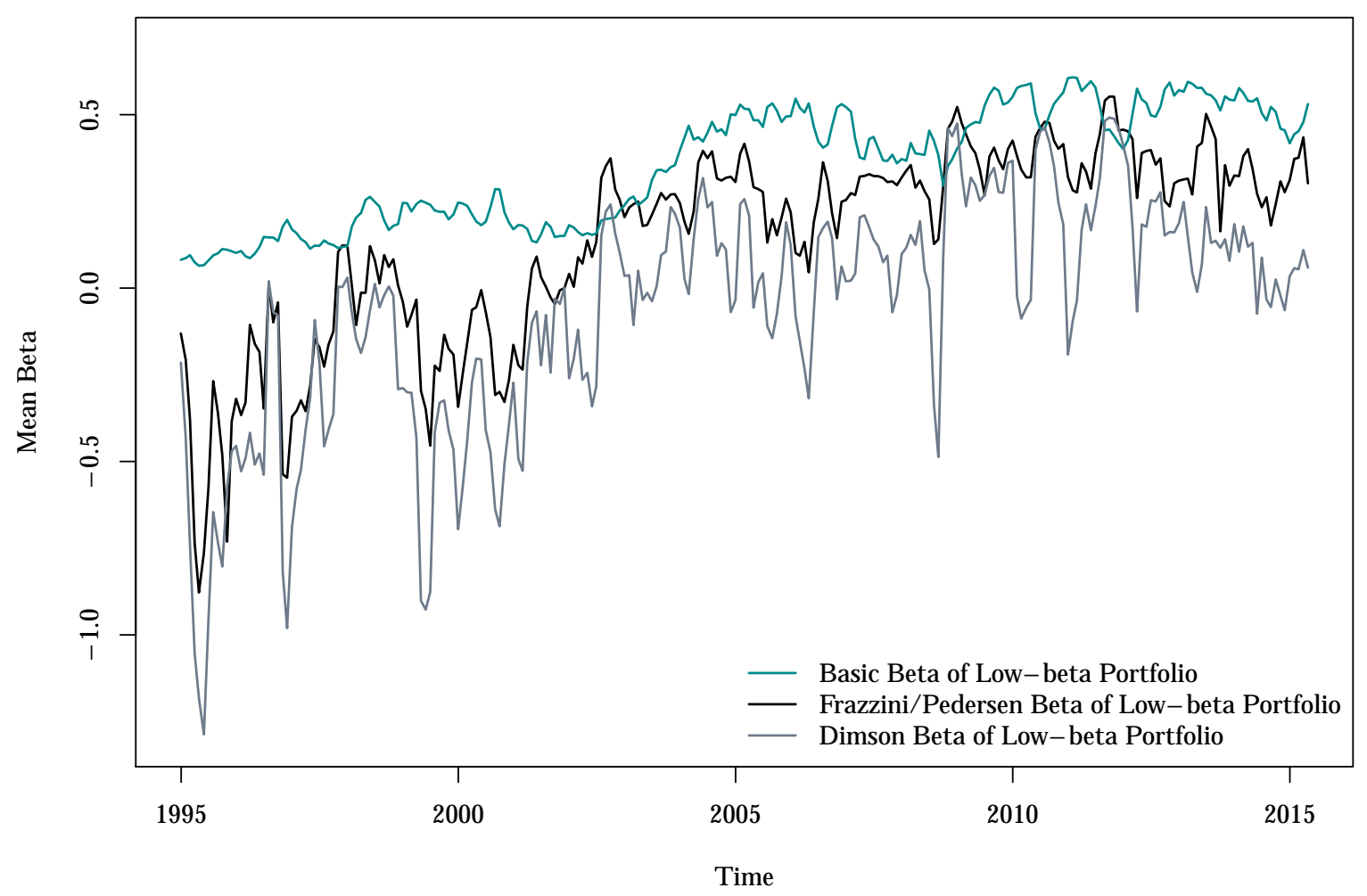

Note: Figure 2.3 shows the effects of the different estimation methods of beta. The displayed graphs are the beta-weighted beta of the low-beta portfolio in the base case scenario for the three different estimation methods. The figure shows substantially different outcomes for the three estimation methods, which also influence the weights of the other instruments invested in.

\footnotetext{
${ }^{15}$ The conclusions from the figure of the high-beta portfolio do not differ, so they are not displayed in this chapter, but are available upon request.
} 
Figure 2.3 indicates, that the estimation method of beta may have substantial influence on the performance, as the portfolio weights of all strategies are based on these mean betas. Table 2.4 shows the performance of the four basic low-beta strategies with the Frazzini/Pedersen estimation method. The average return is higher for the EH strategy and lower for the EL strategy compared to the base case. Because of that, the return of the strategies are much more aligned. The standard deviation of all four strategies is lower, so that the Sharpe ratio for the strategies is more favorable compared to the base case. The same can be concluded from the CEV.

In contrast to the base case, the risk-adjusted returns are significant and positive for all strategies. The EH strategy yields the highest risk-adjusted return, followed by BL, NM and EL. The ranking of the four low-beta strategies is completely reversed compared to the base case. In addition, the EH and BL strategies have a clear negative exposure towards the size factor and the NM and EL strategies have a positive exposure towards the value factor. The EH strategy is the only one that has no significant market exposure. These results differ substantially from the base case in various ways. The strategies with basic beta estimation have an exposure towards the momentum factor; only the NM and EL strategies produce significant positive risk-adjusted returns; and the EH and BL strategies have a significant positive market exposure. The more stable beta estimation with the Frazzini/Pedersen method is reflected in more steady returns of the resulting strategies. As shown, the average returns are more aligned, the standard deviation is lower and the risk-adjusted returns are significant and in reverse ordering for the four low-beta strategies. This might be due to the less varying mean beta of the low-beta and high-beta portfolio which has a direct influence on the weights of all instruments. 
Table 2.4: Estimation Method: Frazzini/Pedersen - Return and Risk

\begin{tabular}{lcccc} 
Panel A: & \multicolumn{4}{c}{$\begin{array}{c}\text { Average Returns, Standard Deviations, Sharpe Ratios } \\
\text { and Certainty }\end{array}$} \\
\hline Equivalent & Returns & \\
\hline Strategy & AvRet & SD & SR & CEV \\
\hline EH & 0.1074 & 0.4300 & 0.2498 & -0.0017 \\
BL & 0.1148 & 0.2495 & 0.4600 & 0.0810 \\
NM & 0.1308 & 0.1959 & 0.6673 & 0.1108 \\
EL & 0.1221 & 0.2016 & 0.6059 & 0.1017
\end{tabular}

Panel B: Risk-adjusted Returns and Factor Sensitivities

\begin{tabular}{lccccc}
\hline Strategy & $\alpha$ & $\beta_{\text {Market }}$ & $\beta_{S M B}$ & $\beta_{H M L}$ & $\beta_{M O M}$ \\
\hline EH & $\mathbf{0 . 1 3 8 4}$ & 0.1919 & $\mathbf{- 2 . 6 9 1 5}$ & 0.2002 & 0.0344 \\
& $(0.0888)$ & $(0.4820)$ & $(0.0003)$ & $(0.7162)$ & $(0.9284)$ \\
BL & $\mathbf{0 . 1 0 8 1}$ & $\mathbf{0 . 3 6 5 4}$ & $\mathbf{- 1 . 1 3 1 7}$ & 0.5757 & -0.0778 \\
& $(0.0649)$ & $(0.0342)$ & $(0.0212)$ & $(0.1307)$ & $(0.7709)$ \\
NM & $\mathbf{0 . 0 8 9 7}$ & $\mathbf{0 . 4 7 5 1}$ & 0.0348 & $\mathbf{0 . 8 5 2 5}$ & -0.1531 \\
& $(0.0228)$ & $(0.0001)$ & $(0.9175)$ & $(0.0002)$ & $(0.3656)$ \\
& & & & & \\
EL & $\mathbf{0 . 0 7 7 8}$ & $\mathbf{0 . 5 3 8 9}$ & 0.4280 & $\mathbf{0 . 9 5 1 2}$ & -0.1900 \\
& $(0.0049)$ & $(0.0000)$ & $(0.1074)$ & $(0.0000)$ & $(0.1107)$ \\
\hline
\end{tabular}

Note: Table 2.4 shows, in Panel A, the annualized average returns (AvRet), standard deviations (SD), Sharpe ratios (SR), and certainty equivalent returns (CEV), and in Panel B, the annualized risk-adjusted returns (alphas) and factor sensitivities in a Carhart (1997) four-factor model for each of the four lowbeta strategies (extreme high (EH), balanced (BL), no market investment (NM), extreme low (EL)). The base case uses the S\&P 1500 stocks as the investible universe, the Frazzini/Pedersen estimation, an estimation period of 3 months for the volatilities, and a 12-month holding period. The strategies are set up at the end of each month for the period December 1994 to April 2015. Each low-beta and high-beta portfolio consists of 150 stocks, which are beta-weighted within the portfolios. The average return is the yearly return earned by each strategy, and the standard deviation is calculated from the returns for the whole investigation period. The Sharpe ratio is calculated by dividing the average return by the standard deviation, and the certainty equivalent return is calculated for an investor with CARA utility function and absolute risk aversion of 1 . The multiple linear regressions underlying the results of Panel B use four independent variables (market excess return, SMB, HML, MOM) and read $R_{t}=\alpha+\beta_{\text {Market }} \cdot\left(R_{M, t}-R_{f, t}\right)+\beta_{S M B} \cdot S M B_{t}+\beta_{H M L} \cdot H M L_{t}+\beta_{M O M} \cdot M O M_{t}+\epsilon_{t}$, where $\left(R_{M, t}-R_{f, t}\right)$ is the excess return of the market proxy at time $t$ and $S M B_{t}, H M L_{t}$ and $M O M_{t}$ are the returns of the factor-mimicking portfolios for size, value, and momentum effects, respectively. The calculations of the p-values (in parentheses) use the Newey-West estimator with 11 lags to account for the overlapping periods. Coefficients that are significant at least at a $10 \%$ level are printed in boldface. 
The average returns of the strategies based on the Dimson method ${ }^{16}$ presented in Table 2.5 are the highest and most aligned of all three estimation methods. Taking the standard deviations into account and comparing the Sharpe ratios of the strategies, the NM and the EL strategy are robust towards the estimation method. The performances of the EH and BL strategy improve even more when the Dimson estimation is used and the NM and EL strategies slightly fall back compared with the Frazzini/Pedersen estimation and the base case.

In contrast to the base case, the EH and the BL strategies obtain positive and significant risk-adjusted returns. Again, the relative advantageousness of the strategies is the exact opposite compared to the base case. All four basic low-beta strategies have a positive market exposure and experience a size exposure that is negative for $\mathrm{EH}$ and $\mathrm{BL}$, but positive for NM and EL. In addition, the NM and EL strategies have an significant value exposure. The returns of the low-beta strategies based on the Dimson method are the ones that are the most exposed to common risk factors compared to all other variations of design elements, but still the strategies overweighting high-beta stocks attain risk-adjusted returns. It is remarkable that the zero ex-ante beta strategies all experience a distinct market exposure. For the Dimson method, the ex-ante beta seem to differ substantially from the ex-post beta in the holding period of the portfolio, which clearly influences the performance of the low-beta strategies. A possible explanation is the more pronounced low and high beta as seen in Figure 2.3. The weights of the different instruments in each strategy depend on the estimated beta and if the betas are more severe, it is not surprising that the performance of the strategy differs markedly.

\footnotetext{
${ }^{16}$ The results and their qualitative interpretation hold if the estimation method is adjusted, so that only backward-looking information is included as described in the previous section.
} 
Table 2.5: Estimation Method: Dimson - Return and Risk

\begin{tabular}{lcccc} 
Panel A: & \multicolumn{4}{c}{$\begin{array}{c}\text { Average Returns, Standard Deviations, Sharpe Ratios } \\
\text { and Certainty }\end{array}$} \\
\hline Equivalent & Returns & \\
\hline Strategy & AvRet & SD & SR & CEV \\
\hline EH & 0.1813 & 0.5593 & 0.3242 & 0.0178 \\
BL & 0.1785 & 0.3823 & 0.4670 & 0.1033 \\
NM & 0.1846 & 0.3284 & 0.5622 & 0.1317 \\
EL & 0.1757 & 0.3265 & 0.5382 & 0.1241 \\
\hline
\end{tabular}

Panel B: Risk-adjusted Returns and Factor Sensitivities

\begin{tabular}{lccccc}
\hline Strategy & $\alpha$ & $\beta_{\text {Market }}$ & $\beta_{S M B}$ & $\beta_{H M L}$ & $\beta_{M O M}$ \\
\hline EH & $\mathbf{0 . 1 3 9 8}$ & $\mathbf{1 . 7 6 8 6}$ & $\mathbf{- 3 . 2 4 9 0}$ & -0.0075 & 0.3056 \\
& $(0.0354)$ & $(0.0000)$ & $(0.0000)$ & $(0.9866)$ & $(0.4859)$ \\
BL & & & & & \\
& $\mathbf{0 . 1 0 6 4}$ & $\mathbf{1 . 6 0 7 8}$ & $\mathbf{- 1 . 3 2 5 7}$ & 0.3696 & 0.1698 \\
& $(0.0533)$ & $(0.0000)$ & $(0.0011)$ & $(0.2338)$ & $(0.6043)$ \\
NM & 0.0729 & $\mathbf{1 . 4 3 2 8}$ & $\mathbf{0 . 5 2 3 2}$ & $\mathbf{0 . 7 4 3 1}$ & 0.0846 \\
& $(0.1088)$ & $(0.0000)$ & $(0.0510)$ & $(0.0001)$ & $(0.7239)$ \\
EL & 0.0729 & $\mathbf{1 . 4 4 7 0}$ & $\mathbf{0 . 5 9 7 5}$ & $\mathbf{0 . 7 4 6 7}$ & 0.0339 \\
& $(0.1032)$ & $(0.0000)$ & $(0.0175)$ & $(0.0000)$ & $(0.8828)$ \\
\hline
\end{tabular}

Note: Table 2.5 shows, in Panel A, the annualized average returns (AvRet), standard deviations (SD), Sharpe ratios (SR), and certainty equivalent returns (CEV), and in Panel B, the annualized risk-adjusted returns (alphas) and factor sensitivities in a Carhart (1997) four-factor model for each of the four low-beta strategies (extreme high (EH), balanced (BL), no market investment (NM), extreme low (EL)). The base case uses the S\&P 1500 stocks as the investible universe, the Dimson estimation, an estimation period of 3 months, and a 12-month holding period. The strategies are set up at the end of each month for the period December 1994 to April 2015. Each low-beta and high-beta portfolio consists of 150 stocks, which are beta-weighted within the portfolios. The average return is the yearly return earned by each strategy, and the standard deviation is calculated from the returns for the whole investigation period. The Sharpe ratio is calculated by dividing the average return by the standard deviation, and the certainty equivalent return is calculated for an investor with CARA utility function and absolute risk aversion of 1 . The multiple linear regressions underlying the results of Panel B use four independent variables (market excess return, SMB, $\mathrm{HML}, \mathrm{MOM})$ and read $R_{t}=\alpha+\beta_{\text {Market }} \cdot\left(R_{M, t}-R_{f, t}\right)+\beta_{S M B} \cdot S M B_{t}+\beta_{H M L} \cdot H M_{t}+\beta_{M O M} \cdot M O M_{t}+\epsilon_{t}$, where $\left(R_{M, t}-R_{f, t}\right)$ is the excess return of the market proxy at time $t$ and $S M B_{t}, H M L_{t}$ and $M O M_{t}$ are the returns of the factor-mimicking portfolios for size, value, and momentum effects, respectively. The calculations of the p-values (in parentheses) use the Newey-West estimator with 11 lags to account for the overlapping periods. Coefficients that are significant at least at a $10 \%$ level are printed in boldface. 
The favorability of the four basic low-beta strategies based on alternative beta estimation methods clearly differs from the base case when comparing average returns as well as risk-adjusted returns. The risk-adjusted returns of the strategies with the alternative estimation methods, seem to be more driven by common risk factors, except momentum. Remarkably, the average returns are more aligned and all four strategies based on the Frazzini/Pedersen estimation are able to generate significant risk-adjusted returns. Summarizing, the estimation method has a substantial impact on the risk and return characteristics of the low-beta strategies. Although significant risk-adjusted returns can be achieved, the performance is much more driven by risk factors.

\subsubsection{Alternative Estimation Periods}

As a second additional design element, we examine the period used to estimate beta. Table 2.6 shows that a shorter estimation period (1 month) results in both higher average returns, higher standard deviations and only small changes in Sharpe ratios and CEVs in comparison with the base case.

Alphas show the same patterns as in the base case, meaning significantly positive alphas when overweighting low-beta stocks in a strategy. The values are generally higher than in the base case. Remarkably, the BL strategy has a significant alpha, which is even higher than the average return, probably due to a strong negative momentum exposure that overcompensates the positive market exposure. Market exposure is again very large for the EH strategy, and momentum exposure is generally higher (more negative) than in the base case. 
Table 2.6: Estimation Period: 1 Month - Return and Risk

\begin{tabular}{lcccc} 
Panel A: & \multicolumn{4}{c}{$\begin{array}{c}\text { Average Returns, Standard Deviations, Sharpe Ratios } \\
\text { and Certainty }\end{array}$} \\
& AvRuivalent & Returns & \\
\hline Strategy & 0.1453 & 0.5887 & 0.2469 & -0.0366 \\
\hline EH & 0.1751 & 0.4347 & 0.4029 & 0.0762 \\
BL & 0.2045 & 0.4087 & 0.5004 & 0.1216 \\
NM & 0.2050 & 0.4114 & 0.4981 & 0.1213 \\
EL & & &
\end{tabular}

Panel B: Risk-adjusted Returns and Factor Sensitivities

\begin{tabular}{lccccc}
\hline Strategy & $\alpha$ & $\beta_{\text {Market }}$ & $\beta_{S M B}$ & $\beta_{H M L}$ & $\beta_{M O M}$ \\
\hline EH & 0.1182 & $\mathbf{1 . 1 3 3 5}$ & -0.0189 & 0.3244 & $\mathbf{- 0 . 8 8 5 4}$ \\
& $(0.3612)$ & $(0.0012)$ & $(0.9857)$ & $(0.6193)$ & $(0.0431)$ \\
BL & & & & \\
& $\mathbf{0 . 1 7 7 9}$ & $\mathbf{0 . 5 2 7 0}$ & -0.1021 & 0.1115 & $\mathbf{- 0 . 6 3 9 7}$ \\
& $(0.0710)$ & $(0.0581)$ & $(0.8852)$ & $(0.8056)$ & $(0.0248)$ \\
NM & & & & -0.0892 & $\mathbf{- 0 . 3 9 8 4}$ \\
& $\mathbf{0 . 2 3 4 9}$ & -0.0373 & -0.1980 & $-0.0591)$ \\
EL & $(0.0100)$ & $(0.9087)$ & $(0.6976)$ & $(0.7790)$ & $(0.059)$ \\
& & & & & -0.3940 \\
\end{tabular}

Note: Table 2.6 shows, in Panel A, the annualized average returns (AvRet), standard deviations (SD), Sharpe ratios (SR), and certainty equivalent returns (CEV), and in Panel B, the annualized risk-adjusted returns (alphas) and factor sensitivities in a Carhart (1997) four-factor model for each of the four low-beta strategies (extreme high (EH), balanced (BL), no market investment (NM), extreme low (EL)). This case uses the S\&P 1500 stocks as the investible universe, the basic estimation method, an estimation period of 1 month, and a 12-month holding period. The strategies are set up at the end of each month for the period December 1994 to April 2015. Each low-beta and high-beta portfolio consists of 150 stocks, which are betaweighted within the portfolios. Average return is the yearly return earned by each strategy and standard deviation is calculated from the returns for the whole investigation period. The Sharpe ratio is calculated by dividing average return by the standard deviation, and the certainty equivalent return is calculated for an investor with CARA utility function and absolute risk aversion of 1. The multiple linear regressions underlying the results of Panel B use four independent variables (market excess return, SMB, HML, $\mathrm{MOM})$ and read $R_{t}=\alpha+\beta_{\text {Market }} \cdot\left(R_{M, t}-R_{f, t}\right)+\beta_{S M B} \cdot S M B_{t}+\beta_{H M L} \cdot H M L_{t}+\beta_{M O M} \cdot M O M_{t}+\epsilon_{t}$, where $\left(R_{M, t}-R_{f, t}\right)$ is the excess return of the market proxy at time $t$ and $S M B_{t}, H M L_{t}$ and $M O M_{t}$ are the returns of the factor-mimicking portfolios for size, value, and momentum effects, respectively. The calculations of the p-values (in parentheses) use the Newey-West estimator with 11 lags to account for the overlapping periods. Coefficients that are significant at least at a $10 \%$ level are printed in boldface. 
Enlarging the estimation period to 12 months leads to a decrease in average returns and standard deviations in line with lower Sharpe ratios and CEVs, as shown in Table 2.7. Hence, this variation in the design of a low-beta strategy does not seem to be favorable. Unreported results (available on request) show that this conclusion still holds true for an even longer estimation period of 36 months. The effects are qualitatively the same as for the 12-month period.

According to Table 2.7, the alphas of the NM and EL strategies are still positive and significant. For the BL and EH strategies, the alphas are much lower, and in the extreme can even be negative. The market exposure is slightly below the level of the base case. A significant size exposure is observable for the EL and NM strategies with positive values. Momentum exposure vanishes gradually when the estimation period is enlarged.

In summary, we find that a shortening of the estimation period results in higher alphas. An enlargement of the period leads to less momentum exposure and significant size exposure for the NM and EL strategies, suggesting that overweighting low-beta stocks while simultaneously enlarging the estimation period leads to increased investment in small stocks. 
Table 2.7: Estimation Period: 12 Months - Return and Risk

\begin{tabular}{lcccc} 
Panel A: & \multicolumn{4}{c}{$\begin{array}{c}\text { Average Returns, Standard Deviations, Sharpe Ratios } \\
\text { and Certainty }\end{array}$} \\
& AvRuivalent & Returns & \\
\hline Strategy & 0.0590 & 0.4480 & 0.1318 & -0.0618 \\
\hline EH & 0.0784 & 0.2713 & 0.2891 & 0.0382 \\
BL & 0.0945 & 0.1949 & 0.4848 & 0.0752 \\
NM & 0.0978 & 0.1979 & 0.4940 & 0.0783 \\
EL & & & &
\end{tabular}

Panel B: Risk-adjusted Returns and Factor Sensitivities

\begin{tabular}{lccccc}
\hline Strategy & $\alpha$ & $\beta_{\text {Market }}$ & $\beta_{S M B}$ & $\beta_{H M L}$ & $\beta_{M O M}$ \\
\hline EH & -0.0140 & $\mathbf{1 . 0 2 4 9}$ & 1.1043 & 0.5125 & $\mathbf{- 0 . 4 9 9 3}$ \\
& $(0.9074)$ & $(0.0003)$ & $(0.2576)$ & $(0.3275)$ & $(0.0370)$ \\
BL & 0.0448 & $\mathbf{0 . 3 9 9 8}$ & 0.9399 & 0.2163 & $\mathbf{- 0 . 3 3 3 6}$ \\
& $(0.5437)$ & $(0.0166)$ & $(0.1288)$ & $(0.5161)$ & $(0.0228)$ \\
NM & $\mathbf{0 . 0 8 7 8}$ & -0.0305 & $\mathbf{0 . 8 0 8 5}$ & 0.0215 & $\mathbf{- 0 . 2 1 1 0}$ \\
& $(0.0518)$ & $(0.8398)$ & $(0.0328)$ & $(0.9291)$ & $(0.0710)$ \\
EL & & & & & \\
& $\mathbf{0 . 1 0 3 6}$ & -0.2253 & $\mathbf{0 . 7 7 5 5}$ & -0.0799 & -0.1680 \\
& $(0.0266)$ & $(0.2403)$ & $(0.0364)$ & $(0.7369)$ & $(0.1052)$ \\
\hline
\end{tabular}

Note: Table 2.7 shows, in Panel A, the annualized average returns (AvRet), standard deviations (SD), Sharpe ratios (SR), and certainty equivalent returns (CEV), and in Panel B, the annualized risk-adjusted returns (alphas) and factor sensitivities in a Carhart (1997) four-factor model for each of the four low-beta strategies (extreme high (EH), balanced (BL), no market investment (NM), extreme low (EL)). This case uses the S\&P 1500 stocks as the investible universe, the basic estimation method, an estimation period of 12 months, and a 12-month holding period. The strategies are set up at the end of each month for the period December 1994 to April 2015. Each low-beta and high-beta portfolio consists of 150 stocks, which are beta-weighted within the portfolios. Average return is the yearly return earned by each strategy and the standard deviation is calculated from the returns for the whole investigation period. The Sharpe ratio is calculated by dividing average return by the standard deviation, and the certainty equivalent return is calculated for an investor with CARA utility function and absolute risk aversion of 1 . The multiple linear regressions underlying the results of Panel B use four independent variables (market excess return, SMB, HML, MOM) and read $R_{t}=\alpha+\beta_{\text {Market }} \cdot\left(R_{M, t}-R_{f, t}\right)+\beta_{S M B} \cdot S M B_{t}+\beta_{H M L} \cdot H M L_{t}+\beta_{M O M} \cdot M O M_{t}+\epsilon_{t}$, where $\left(R_{M, t}-R_{f, t}\right)$ is the excess return of the market proxy at time $t$ and $S M B_{t}, H M L_{t}$ and $M O M_{t}$ are the returns of the factor-mimicking portfolios for size, value, and momentum effects, respectively. The calculations of the p-values (in parentheses) use the Newey-West estimator with 11 lags to account for the overlapping periods. Coefficients that are significant at least at a $10 \%$ level are printed in boldface. 


\subsubsection{Varying Portfolio Coverage}

Our next analysis considers the effects of varying portfolio coverage. Smaller coverage (30 stocks) results in higher average returns than in the base case, but also higher standard deviations. In terms of the risk-return trade-off, the latter effect overcompensates the former, leading to lower Sharpe ratios and CEVs in all but one case. The only (slight) improvement refers to the Sharpe ratio of the EH strategy. However, the EH strategy is still the worst of all strategies. As Table 2.8 shows, the relative ranking of different strategies is unchanged compared to the base case when using smaller portfolio coverage. Very similar patterns as in the base case are found for the alphas and factor sensitivities. There is one interesting difference, however. On the one hand, the values of the alphas and factor sensitivities are generally higher (in absolute terms) than in the base case. On the other hand, statistical significance declines, because returns become more noisy over time. In particular, the alphas of the various strategies are no longer statistically significant. This is very much in line with the increased average returns but lower Sharpe ratios as compared with the base case. 
Table 2.8: Portfolio Coverage: 2\% (30 Stocks) - Return and Risk

\begin{tabular}{lcccc} 
Panel A: & \multicolumn{4}{c}{$\begin{array}{c}\text { Average Returns, Standard Deviations, Sharpe Ratios } \\
\text { and Certainty }\end{array}$} \\
& AvRuivalent & Returns & \\
\hline Strategy & 0.1521 & 0.7229 & 0.2105 & -0.1482 \\
\hline EH & 0.1580 & 0.4721 & 0.3347 & 0.0407 \\
BL & 0.1644 & 0.4018 & 0.4091 & 0.0889 \\
NM & 0.1638 & 0.4061 & 0.4035 & 0.0871 \\
EL & & & & \\
\end{tabular}

Panel B: Risk-adjusted Returns and Factor Sensitivities

\begin{tabular}{lccccc}
\hline Strategy & $\alpha$ & $\beta_{\text {Market }}$ & $\beta_{S M B}$ & $\beta_{H M L}$ & $\beta_{M O M}$ \\
\hline EH & 0.0885 & $\mathbf{1 . 4 7 2 2}$ & 0.5575 & 0.4302 & $\mathbf{- 0 . 8 7 5 6}$ \\
& $(0.6308)$ & $(0.0002)$ & $(0.7235)$ & $(0.5913)$ & $(0.0494)$ \\
BL & 0.1286 & $\mathbf{0 . 6 9 3 9}$ & 0.3426 & 0.3870 & $\mathbf{- 0 . 5 5 9 3}$ \\
& $(0.3346)$ & $(0.0239)$ & $(0.7513)$ & $(0.5104)$ & $(0.0307)$ \\
NM & 0.1680 & -0.0370 & -0.1044 & 0.3445 & -0.2598 \\
& $(0.1523)$ & $(0.9300)$ & $(0.8341)$ & $(0.3780)$ & $(0.1209)$ \\
EL & & & & & \\
& 0.1688 & -0.0844 & 0.1277 & 0.3437 & -0.2429 \\
& $(0.1586)$ & $(0.8510)$ & $(0.7976)$ & $(0.3970)$ & $(0.1537)$ \\
\hline
\end{tabular}

Note: Table 2.8 shows, in Panel A, the annualized average returns (AvRet), standard deviations (SD), Sharpe ratios (SR), and certainty equivalent returns (CEV), and in Panel B, the annualized risk-adjusted returns (alphas) and factor sensitivities in a Carhart (1997) four-factor model for each of the four low-beta strategies (extreme high (EH), balanced (BL), no market investment (NM), extreme low (EL)). This case uses the S\&P 1500 stocks as the investible universe, the basic estimation method, an estimation period of 3 months, and a 12-month holding period. The strategies are set up at the end of each month for the period December 1994 to April 2015. Each low-beta and high-beta portfolio consists of 30 stocks, which are beta-weighted within the portfolios. The average return is the yearly return earned by each strategy and the standard deviation is calculated from the returns for the whole investigation period. The Sharpe ratio is calculated by dividing average return by the standard deviation, and the certainty equivalent return is calculated for an investor with CARA utility function and absolute risk aversion of 1 . The multiple linear regressions underlying the results of Panel B use four independent variables (market excess return, SMB, HML, MOM) and read $R_{t}=\alpha+\beta_{\text {Market }} \cdot\left(R_{M, t}-R_{f, t}\right)+\beta_{S M B} \cdot S M B_{t}+\beta_{H M L} \cdot H M L_{t}+\beta_{M O M} \cdot M O M_{t}+\epsilon_{t}$, where $\left(R_{M, t}-R_{f, t}\right)$ is the excess return of the market proxy at time $t$ and $S M B_{t}, H M L_{t}$ and $M O M_{t}$ are the returns of the factor-mimicking portfolios for size, value, and momentum effects, respectively. The calculations of the p-values (in parentheses) use the Newey-West estimator with 11 lags to account for the overlapping periods. Coefficients that are significant at least at a $10 \%$ level are printed in boldface. 
An increase in portfolio coverage (300 stocks) leads to slightly lower average returns and standard deviations than in the base case, as shown in Table 2.9. The consequences for the risk-return trade-off depend on the strategy and the criterion (Sharpe ratio or CEV). With respect to the Sharpe ratio, we observe somewhat higher values for the NM and EL strategies.

The patterns in the alphas and the factor sensitivities are similar to the base case. One difference is that the momentum exposure is somewhat diminished and the EL strategy has a significant size exposure. With respect to alphas, we observe that their magnitude is going down, but the p-values for the NM and EL strategies are also lower. This finding suggests that there is a trade-off between realizing a higher alpha and being able to establish its statistical significance when changing the portfolio coverage. Smaller coverage, that is, concentrating on stocks with more extreme betas, seems to produce higher (raw and risk-adjusted) average returns, but also much higher (raw and idiosyncratic) return volatility. These general effects of portfolio coverage for the low-beta strategies are well in line with the results by Amenc et al. (2016), who study the tilting of an index towards six different factors. Their study also concludes that a too narrow coverage does not achieve a satisfactory degree of diversification, leading to unnecessarily high risk levels. 
Table 2.9: Portfolio Coverage: 20\% (300 Stocks) - Return and Risk

\begin{tabular}{lcccc} 
Panel A: & \multicolumn{4}{c}{$\begin{array}{l}\text { Average Returns, Standard Deviations, Sharpe Ratios } \\
\text { and Certainty }\end{array}$} \\
& Equivalent Returns & \\
\hline Strategy & AvRet & SD & SR & CEV \\
\hline EH & 0.0534 & 0.3916 & 0.1365 & -0.0300 \\
BL & 0.0893 & 0.2383 & 0.3748 & 0.0597 \\
NM & 0.1223 & 0.1987 & 0.6156 & 0.1025 \\
EL & 0.1252 & 0.2116 & 0.5914 & 0.1031 \\
\hline
\end{tabular}

Panel B: Risk-adjusted Returns and Factor Sensitivities

\begin{tabular}{lccccc}
\hline Strategy & $\alpha$ & $\beta_{\text {Market }}$ & $\beta_{S M B}$ & $\beta_{H M L}$ & $\beta_{M O M}$ \\
\hline EH & 0.0093 & $\mathbf{0 . 8 7 7 3}$ & 0.3545 & 0.3654 & -0.4730 \\
& $(0.9262)$ & $(0.0007)$ & $(0.6695)$ & $(0.4355)$ & $(0.1022)$ \\
BL & 0.0686 & $\mathbf{0 . 3 6 3 8}$ & 0.4383 & 0.1704 & $\mathbf{- 0 . 2 9 0 2}$ \\
& $(0.2760)$ & $(0.0165)$ & $(0.4007)$ & $(0.5218)$ & $(0.0491)$ \\
NM & $\mathbf{0 . 1 1 8 0}$ & 0.0014 & 0.4800 & -0.0050 & -0.1360 \\
& $(0.0197)$ & $(0.9937)$ & $(0.1207)$ & $(0.9779)$ & $(0.2437)$ \\
EL & & & & & \\
& $\mathbf{0 . 1 2 7 9}$ & -0.1497 & $\mathbf{0 . 5 2 2 0}$ & -0.0246 & -0.1073 \\
& $(0.0248)$ & $(0.4821)$ & $(0.0831)$ & $(0.8917)$ & $(0.3386)$ \\
\hline
\end{tabular}

Note: Table 2.9 shows, in Panel A, annualized average returns (AvRet), standard deviations (SD), Sharpe ratios (SR), and certainty equivalent returns (CEV), and in Panel B, annualized risk-adjusted returns (alphas) and factor sensitivities in a Carhart (1997) four-factor model for each of the four low-beta strategies (extreme high (EH), balanced (BL), no market investment (NM), extreme low (EL)). This case uses the S\&P 1500 stocks as the investible universe, the basic estimation method, an estimation period of 3 months, and a 12-month holding period. The strategies are set up at the end of each month for the period December 1994 to April 2015. Each low-beta and high-beta portfolio consists of 300 stocks, which are beta-weighted within the portfolios. The average return is the yearly return earned by each strategy and the standard deviation is calculated from the returns for the whole investigation period. The Sharpe ratio is calculated by dividing average return by the standard deviation, and the certainty equivalent return is calculated for an investor with CARA utility function and absolute risk aversion of 1 . The multiple linear regressions underlying the results of Panel B use four independent variables (market excess return, SMB, HML, $\mathrm{MOM})$ and read $R_{t}=\alpha+\beta_{\text {Market }} \cdot\left(R_{M, t}-R_{f, t}\right)+\beta_{S M B} \cdot S M B_{t}+\beta_{H M L} \cdot H M L_{t}+\beta_{M O M} \cdot M O M_{t}+\epsilon_{t}$, where $\left(R_{M, t}-R_{f, t}\right)$ is the excess return of the market proxy at time $t$ and $S M B_{t}, H M L_{t}$ and $M O M_{t}$ are the returns of the factor-mimicking portfolios for size, value and momentum effects, respectively. The calculations of the p-values (in parentheses) use the Newey-West estimator with 11 lags to account for the overlapping periods. Coefficients that are significant at least at a $10 \%$ level are printed in boldface. 


\subsubsection{Alternative Weighting within Portfolios}

As shown in Table 2.10, an equal weighting of the constituents of the low-beta and high-beta portfolios leads to similar results regarding risk and return characteristics as the beta weighting. Average returns are slightly lower and, at the same time, the standard deviations are slightly lower as well, leading to very similar Sharpe ratios and CEVs compared to Table 2.3.

The alphas are somewhat lower compared to the base case, but again the NM and the EL strategies lead to significant, positive alphas and have no significant market exposure, whereas the EH and BL strategies show insignificant alphas and a significant positive market exposure. Again, no significant size or value factor loadings are observed for any strategy, but there are still significant negative momentum factor exposures, which become stronger the more high-beta stocks are overweighted. 


\section{Table 2.10: Weighting within the Portfolios: Equal Weighting - Return and Risk}

\begin{tabular}{lcccc} 
Panel A: & \multicolumn{4}{c}{$\begin{array}{l}\text { Average Returns, Standard Deviations, Sharpe Ratios } \\
\text { and Certainty }\end{array}$} \\
& Equivalent Returns & \\
\hline Strategy & AvRet & SD & SR & CEV \\
\hline EH & 0.0808 & 0.4671 & 0.1730 & -0.0433 \\
BL & 0.1036 & 0.2844 & 0.3644 & 0.0610 \\
NM & 0.1282 & 0.2204 & 0.5815 & 0.1039 \\
EL & 0.1265 & 0.2290 & 0.5522 & 0.1007 \\
\hline
\end{tabular}

Panel B: Risk-adjusted Returns and Factor Sensitivities

\begin{tabular}{lccccc}
\hline Strategy & $\alpha$ & $\beta_{\text {Market }}$ & $\beta_{S M B}$ & $\beta_{H M L}$ & $\beta_{M O M}$ \\
\hline EH & 0.0260 & $\mathbf{0 . 9 8 2 3}$ & 0.6701 & 0.4031 & $\mathbf{- 0 . 5 4 5 0}$ \\
& $(0.8216)$ & $(0.0009)$ & $(0.4803)$ & $(0.4577)$ & $(0.0895)$ \\
BL & 0.0801 & $\mathbf{0 . 4 1 9 3}$ & 0.5656 & 0.1922 & $\mathbf{- 0 . 3 6 5 9}$ \\
& $(0.2383)$ & $(0.0144)$ & $(0.3080)$ & $(0.5061)$ & $(0.0393)$ \\
NM & $\mathbf{0 . 1 3 1 2}$ & -0.0251 & 0.4362 & -0.0026 & $\mathbf{- 0 . 2 2 3 0}$ \\
& $(0.0181)$ & $(0.8965)$ & $(0.1620)$ & $(0.9878)$ & $(0.0631)$ \\
EL & & & & & \\
& $\mathbf{0 . 1 3 4 3}$ & -0.1437 & 0.4611 & -0.0187 & $\mathbf{- 0 . 1 8 6 8}$ \\
& $(0.0260)$ & $(0.5171)$ & $(0.1320)$ & $(0.9099)$ & $(0.0861)$ \\
\hline
\end{tabular}

Note: Table 2.10 shows, in Panel A, the annualized average returns (AvRet), standard deviations (SD), Sharpe ratios (SR), and certainty equivalent returns (CEV), and in Panel B, the annualized risk-adjusted returns (alphas) and factor sensitivities in a Carhart (1997) four-factor model for each of the four low-beta strategies (extreme high (EH), balanced (BL), no market investment (NM), extreme low (EL)). This case uses the S\&P 1500 stocks as the investible universe, the basic estimation method, an estimation period of 3 months, and a 12-month holding period. The strategies are set up at the end of each month for the period December 1994 to April 2015. Each low-beta and high-beta portfolio consists of 150 stocks, which are equal-weighted within the portfolios. The average return is the yearly return earned by each strategy, and the standard deviation is calculated from the returns for the whole investigation period. The Sharpe ratio is calculated by dividing the average return by the standard deviation, and the certainty equivalent return is calculated for an investor with CARA utility function and absolute risk aversion of 1 . The multiple linear regressions underlying the results of Panel B use four independent variables (market excess return, SMB, HML, MOM) and read $R_{t}=\alpha+\beta_{\text {Market }} \cdot\left(R_{M, t}-R_{f, t}\right)+\beta_{S M B} \cdot S M B_{t}+\beta_{H M L} \cdot H M L_{t}+\beta_{M O M} \cdot M O M_{t}+\epsilon_{t}$, where $\left(R_{M, t}-R_{f, t}\right)$ is the excess return of the market proxy at time $t$ and $S M B_{t}, H M L_{t}$ and $M O M_{t}$ are the returns of the factor-mimicking portfolios for size, value and momentum effects, respectively. The calculations of the p-values (in parentheses) use the Newey-West estimator with 11 lags to account for the overlapping periods. Coefficients that are significant at least at a $10 \%$ level are printed in boldface. 
Value weighting within the portfolios leads to substantial differences in all observed patterns, as shown in Table 2.11. In this case, average returns are the highest for the EH strategy and the lowest for the EL strategy. The level of returns is comparable, but in reverse order. This finding suggests that larger stocks within the high-beta portfolio have higher returns than smaller stocks within this portfolio. For the low-beta portfolio, exactly the opposite is true. This result suggests that the good performance of the EL strategy in the base case relies on relatively small stocks within the low-beta portfolio. Standard deviations are in the same order as in the base case (lowest for the EL strategy), and generally even lower. Caused by this pattern, Sharpe ratios are more homogeneous among the different low-beta strategies. For the EH and BL strategies, the Sharpe ratio is clearly improved; for the NM and EL strategies, it is reduced. When judging the risk-return trade-off via the CEVs, we find the same ordering as for average returns.

With value weighting, alphas are positive and significant for all strategies in descending order from EH to EL. In contrast to the base case, no significant market exposure remains, but the EH strategy shows a significant positive size exposure, that is, overweighting high-beta stocks tends to select small stocks. Again the momentum factor exposure is negative for all four strategies but seems to be lower than in the base case for the EH and BL strategies. Whereas an equal weighting leads to only small deviations from the base case results regarding the risk and return characteristics, value weighting within portfolios causes substantial differences. With respect to risk-adjusted returns, beta weighting should be preferred when overweighting low-beta stocks. When overweighting high-beta stocks, value weighting leads to better results.

In summary, the weighting of stocks is very important for the performance of low-beta strategies. We show that not only does the relative weighting of low-beta and high-beta stocks lead to substantial differences in average returns and risk characteristics of the resulting strategies, the weighting within the low-beta and high-beta portfolios does as well. This finding complements results on smart beta strategies using different weighting schemes within an investible universe. With respect to the base case results, we have learned that relatively small stocks within the low-beta portfolio contribute a lot to the good performance of the EL and NM strategies. 


\section{Table 2.11: Weighting within the Portfolios: Value Weighting - Return and Risk}

\begin{tabular}{lcccc} 
Panel A: & \multicolumn{4}{c}{$\begin{array}{l}\text { Average Returns, Standard Deviations, Sharpe Ratios } \\
\text { and Certainty }\end{array}$} \\
& Equivalent Returns & \\
\hline Strategy & AvRet & SD & SR & CEV \\
\hline EH & 0.1316 & 0.3246 & 0.4055 & 0.0781 \\
BL & 0.1059 & 0.2404 & 0.4405 & 0.0765 \\
NM & 0.0913 & 0.2210 & 0.4130 & 0.0670 \\
EL & 0.0802 & 0.2169 & 0.3696 & 0.0571 \\
\hline
\end{tabular}

Panel B: Risk-adjusted Returns and Factor Sensitivities

\begin{tabular}{lccccc}
\hline Strategy & $\alpha$ & $\beta_{\text {Market }}$ & $\beta_{S M B}$ & $\beta_{H M L}$ & $\beta_{M O M}$ \\
\hline EH & $\mathbf{0 . 1 2 5 6}$ & 0.1422 & $\mathbf{1 . 1 0 2 7}$ & -0.2509 & $\mathbf{- 0 . 3 9 6 1}$ \\
& $(0.0334)$ & $(0.5270)$ & $(0.0513)$ & $(0.4642)$ & $(0.0624)$ \\
BL & & & & \\
& $\mathbf{0 . 1 0 5 1}$ & 0.1035 & 0.7240 & -0.2286 & $\mathbf{- 0 . 2 9 8 0}$ \\
& $(0.0297)$ & $(0.5377)$ & $(0.1168)$ & $(0.3156)$ & $(0.0592)$ \\
NM & & & & -0.2317 & -0.2403 \\
& $(0.0323)$ & $(0.6200)$ & $(0.2582)$ & $(0.1235)$ & $(0.1100)$ \\
EL & & & & -0.2063 & -0.1999 \\
& $\mathbf{0 . 0 8 4 7}$ & 0.0647 & 0.3454 & $(0.1540)$ & $(0.1390)$ \\
\hline
\end{tabular}

Note: Table 2.11 shows, in Panel A, the annualized average returns (AvRet), standard deviations (SD), Sharpe ratios (SR), and certainty equivalent returns (CEV), and in Panel B, the annualized risk-adjusted returns (alphas) and factor sensitivities in a Carhart (1997) four-factor model for each of the four low-beta strategies (extreme high (EH), balanced (BL), no market investment (NM), extreme low (EL)). This case uses the S\&P 1500 stocks as the investible universe, the basic estimation method, an estimation period of 3 months, and a 12-month holding period. The strategies are set up at the end of each month for the period December 1994 to April 2015. Each low-beta and high-beta portfolio consists of 150 stocks, which are value-weighted within the portfolios. The average return is the yearly return earned by each strategy, and the standard deviation is calculated from the returns for the whole investigation period. The Sharpe ratio is calculated by dividing average return by the standard deviation, and the certainty equivalent return is calculated for an investor with CARA utility function and absolute risk aversion of 1 . The multiple linear regressions underlying the results of Panel B use four independent variables (market excess return, SMB, $\mathrm{HML}, \mathrm{MOM})$ and read $R_{t}=\alpha+\beta_{\text {Market }} \cdot\left(R_{M, t}-R_{f, t}\right)+\beta_{S M B} \cdot S M B_{t}+\beta_{H M L} \cdot H M_{t}+\beta_{M O M} \cdot M O M_{t}+\epsilon_{t}$, where $\left(R_{M, t}-R_{f, t}\right)$ is the excess return of the market proxy at time $t$ and $S M B_{t}, H M L_{t}$ and $M O M_{t}$ are the returns of the factor-mimicking portfolios for size, value, and momentum effects, respectively. The calculations of the p-values (in parentheses) use the Newey-West estimator with 11 lags to account for the overlapping periods. Coefficients that are significant at least at a $10 \%$ level are printed in boldface. 


\subsubsection{Alternative Rebalancing Frequencies}

Another important aspect is whether more frequent rebalancing could further improve the low-beta strategies. This is generally not the case, as can be seen from Table 2.12, which show the results for monthly rebalancing. For the EH and BL strategies, Sharpe ratios and CEVs somehow improve, but are still lowest among the four strategies. For the NM and EL strategies, Sharpe ratios and CEVs even decrease.

The alphas show a similar picture to the Sharpe ratios and the CEVs. They somehow increase for the EH and BL strategies, although these alphas remain statistically insignificant. The significant positive alphas of the NM and EL strategies, however, become smaller as compared to the base case. With respect to the other factors, we find that all factor loadings are now insignificant for all strategies. Overall, taking into account that more frequent rebalancing leads to higher transaction costs, our analysis suggests that there is no good reason to rebalance the portfolios more frequently than once per year. ${ }^{17}$

Summarizing our findings on the design variations that a portfolio manager could make, we observe that the most far-reaching impact on the performance of low-beta strategies is a change in the estimation method away from the basic method and a change from a beta weighting or equal weighting of the stocks within the portfolios to a value weighting. All other design elements have an impact on the risk and return characteristics as well, but do not change the relative ranking of the basic strategies if we consider the NM and EL strategies as one group of strategies with very similar characteristics. Usually, these two strategies also have the best Sharpe ratios, CEVs, and alphas. It is nonetheless important to understand possible interdependencies between overweighting low-beta stocks or high-beta stocks and other design elements. In the end, portfolio managers must know what kind of portfolio characteristics they are creating via the choices they make.

\footnotetext{
${ }^{17}$ This statement also applies for a medium frequent rebalancing of 3 months, which leads to very similar results as the monthly rebalancing. Results are therefore not reported and are available upon request.
} 
Table 2.12: Rebalancing: Monthly - Return and Risk

\begin{tabular}{lcccc} 
Panel A: & \multicolumn{4}{c}{$\begin{array}{c}\text { Average Returns, Standard Deviations, Sharpe Ratios } \\
\text { and Certainty }\end{array}$} \\
& AvRuivalent & Returns & \\
\hline Strategy & 0.0859 & 0.3899 & 0.2204 & 0.0048 \\
\hline EH & 0.1068 & 0.2518 & 0.4240 & 0.0740 \\
BL & 0.1302 & 0.2458 & 0.5297 & 0.0996 \\
NM & 0.1276 & 0.2495 & 0.5115 & 0.0960 \\
EL & & & & \\
\end{tabular}

Panel B: Risk-adjusted Returns and Factor Sensitivities

\begin{tabular}{lccccc}
\hline Strategy & $\alpha$ & $\beta_{\text {Market }}$ & $\beta_{S M B}$ & $\beta_{H M L}$ & $\beta_{M O M}$ \\
\hline EH & 0.0672 & -0.0052 & 0.2538 & 0.3108 & 0.0576 \\
& $(0.4609)$ & $(0.9769)$ & $(0.2749)$ & $(0.2120)$ & $(0.6994)$ \\
BL & 0.0936 & 0.0013 & 0.1776 & 0.2567 & 0.0147 \\
& $(0.1138)$ & $(0.9908)$ & $(0.2361)$ & $(0.1102)$ & $(0.8787)$ \\
NM & $\mathbf{0 . 1 2 1 9}$ & 0.0053 & 0.0996 & 0.2043 & -0.0138 \\
& $(0.0360)$ & $(0.9629)$ & $(0.4968)$ & $(0.1935)$ & $(0.8834)$ \\
EL & $\mathbf{0 . 1 2 0 0}$ & 0.0079 & 0.1014 & 0.2026 & -0.0282 \\
& $(0.0422)$ & $(0.9455)$ & $(0.4955)$ & $(0.2037)$ & $(0.7674)$ \\
\hline
\end{tabular}

Note: Table 2.12 shows, in Panel A, the annualized average returns (AvRet), standard deviations (SD), Sharpe ratios (SR), and certainty equivalent returns (CEV), and in Panel B, the annualized risk-adjusted returns (alphas) and factor sensitivities in a Carhart (1997) four-factor model for each of the four low-beta strategies (extreme high (EH), balanced (BL), no market investment (NM), extreme low (EL)). This case uses the S\&P 1500 stocks as the investible universe, the basic estimation method, an estimation period of 3 months, and a 1-month holding period. The strategies are set up at the end of each month for the period December 1994 to April 2015. Each low-beta and high-beta portfolio consists of 150 stocks, which are betaweighted within the portfolios. The average return is the annualized monthly return earned by each strategy and the standard deviation is calculated from the returns for the whole investigation period. The Sharpe ratio is calculated by dividing average return by the standard deviation and the certainty equivalent return is calculated for an investor with CARA utility function and absolute risk aversion of 1 . The multiple linear regressions underlying the results of Panel B use four independent variables (market excess return, SMB, HML, MOM) and read $R_{t}=\alpha+\beta_{\text {Market }} \cdot\left(R_{M, t}-R_{f, t}\right)+\beta_{S M B} \cdot S M B_{t}+\beta_{H M L} \cdot H M L_{t}+\beta_{M O M} \cdot M O M_{t}+\epsilon_{t}$, where $\left(R_{M, t}-R_{f, t}\right)$ is the excess return of the market proxy at time $t$ and $S M B_{t}, H M L_{t}$ and $M O M_{t}$ are the returns of the factor-mimicking portfolios for size, value, and momentum effects, respectively. The calculations of the p-values (in parentheses) use the White estimator to account for heteroscedasticity. Coefficients that are significant at least at a $10 \%$ level are printed in boldface. 


\subsubsection{Using a Smaller Investible Universe}

After analyzing the impact of various design variations for the S\&P 1500 stocks, we repeat the whole analysis for a smaller investible universe, the S\&P 500 Index. As argued in Section 2.2.3, the decision on the investible universe or benchmark is typically made by the asset management firm or the client, not the portfolio manager. Our analysis has shown that for the S\&P 1500 universe, the portfolio manager's decisions are very important for the characteristics of a low-beta strategy. It is an interesting question whether this is still the case for the smaller universe of 500 stocks. An advantage of the smaller universe is that only the most liquid stocks are considered, and therefore transaction costs should be smaller. However, the low-beta effects might be smaller as well and the portfolio manager's choice variables might not suffice to create value via low-beta strategies.

Table 2.13 shows the base case results for the S\&P 500 universe. Average returns are generally smaller than for the larger stock universe, but there is no difference in the relative ranking of the four strategies. Still, NM is the most promising strategy, followed by EL, BL, and EH. The standard deviations are lower as well, but not enough to result in higher Sharpe ratios compared to the larger universe. The same statement applies to the CEV. Unreported results for variations in the various design elements show no advantage from more frequent rebalancing and decreasing average returns with the length of the estimation period.

Risk-adjusted average returns (alphas) are even smaller than the average raw returns, and it is remarkable that this result holds for all four strategies. Moreover, there is no clear ranking among the strategies, because alpha is not statistically significant for any of them. This is a very striking difference compared to the results for the S\&P 1500 universe. It seems that the opportunity to create excess returns through low-beta strategies depends on the availability of the 1000 smaller stocks within the S\&P 1500 index. $^{18}$

\footnotetext{
${ }^{18}$ At first sight this result seems to be at odds with Auer and Schuhmacher (2015), who find a low-beta effect even in the universe of DJIA stocks. However, Auer and Schuhmacher (2015) use a sample period from 1926 to 2013, but do not show alphas for the more recent sub-periods corresponding to our sample period.
} 
Table 2.13: Investible Universe: Smaller Universe - Return and Risk

\begin{tabular}{lcccc} 
Panel A: & \multicolumn{4}{c}{$\begin{array}{c}\text { Average Returns, Standard Deviations, Sharpe Ratios } \\
\text { and Certainty }\end{array}$} \\
\hline Equivalent & Returns & \\
\hline Strategy & AvRet & SD & SR & CEV \\
\hline EH & 0.0338 & 0.4093 & 0.0825 & -0.0622 \\
BL & 0.0622 & 0.2644 & 0.2352 & 0.0246 \\
NM & 0.0994 & 0.2178 & 0.4563 & 0.0756 \\
EL & 0.0906 & 0.2053 & 0.4414 & 0.0691 \\
\hline
\end{tabular}

Panel B: Risk-adjusted Returns and Factor Sensitivities

\begin{tabular}{lccccc}
\hline Strategy & $\alpha$ & $\beta_{\text {Market }}$ & $\beta_{S M B}$ & $\beta_{H M L}$ & $\beta_{M O M}$ \\
\hline EH & 0.0129 & $\mathbf{0 . 6 8 1 5}$ & $\mathbf{- 2 . 0 8 8 0}$ & 0.4042 & 0.1334 \\
& $(0.8395)$ & $(0.0005)$ & $(0.0014)$ & $(0.2890)$ & $(0.6964)$ \\
BL & & & & \\
& 0.0232 & $\mathbf{0 . 6 2 0 8}$ & $\mathbf{- 1 . 0 1 2 7}$ & $\mathbf{0 . 5 5 4 7}$ & 0.0252 \\
& $(0.6036)$ & $(0.0000)$ & $(0.0212)$ & $(0.0232)$ & $(0.9182)$ \\
NM & & & & & -0.0703 \\
& $(0.0206$ & $\mathbf{0 . 6 7 9 3}$ & -0.0836 & $\mathbf{0 . 7 7 0 3}$ & $(0.6508)$ \\
EL & $(0.0000)$ & $(0.7576)$ & $(0.0000)$ & -0.0831 \\
& $(0.0336)$ & $\mathbf{0 . 5 6 0 1}$ & 0.0625 & $\mathbf{0 . 7 0 5 2}$ & $(0.5939)$ \\
\hline
\end{tabular}

Note: Table 2.13 shows, in Panel A, the annualized average returns (AvRet), standard deviations (SD), Sharpe ratios (SR), and certainty equivalent returns (CEV), and in Panel B, the annualized risk-adjusted returns (alphas) and factor sensitivities in a Carhart (1997) four-factor model for each of the four low-beta strategies (extreme high $(\mathrm{EH})$, balanced $(\mathrm{BL})$, no market investment (NM), extreme low (EL)). This case uses the S\&P 500 stocks as the investible universe, the basic estimation method, an estimation period of 3 months, and a 12-month holding period. The strategies are set up at the end of each month for the period December 1994 to April 2015. Each low-beta and high-beta portfolio consists of 50 stocks, which are beta-weighted within the portfolios. The average return is the yearly return earned by each strategy, and the standard deviation is calculated from the returns for the whole investigation period. The Sharpe ratio is calculated by dividing average return by the standard deviation, and the certainty equivalent return is calculated for an investor with CARA utility function and absolute risk aversion of 1 . The multiple linear regressions underlying the results of Panel B use four independent variables (market excess return, SMB, $\mathrm{HML}, \mathrm{MOM})$ and read $R_{t}=\alpha+\beta_{\text {Market }} \cdot\left(R_{M, t}-R_{f, t}\right)+\beta_{S M B} \cdot S M B_{t}+\beta_{H M L} \cdot H M L_{t}+\beta_{M O M} \cdot M O M_{t}+\epsilon_{t}$, where $\left(R_{M, t}-R_{f, t}\right)$ is the excess return of the market proxy at time $t$ and $S M B_{t}, H M L_{t}$ and $M O M_{t}$ are the returns of the factor-mimicking portfolios for size, value and momentum effects, respectively. The calculations of the p-values (in parentheses) use the Newey-West estimator with 11 lags to account for the overlapping periods. Coefficients that are significant at least at a $10 \%$ level are printed in boldface. 
All strategies have a highly significant and positive market exposure. Despite setting up all portfolios as zero ex-ante beta portfolios, the zero-beta condition holds for the beta estimates obtained from the estimation period returns, not necessarily for the realized betas over the holding period. This result is also in contrast to the results for the larger index, except for variations in the estimation method, because a significant market exposure is observed only for the EH and BL strategies. For the S\&P 500 universe, we also find some significant size and value exposures. ${ }^{19}$ The size exposure is more negative and more significant the more high-beta stocks are overweighted. Unreported results show that this finding also holds for different estimation periods and rebalancing frequencies. For the value exposure, the exact opposite occurs: the more low-beta stocks are overweighted, the higher and more significant is the value factor loading of the corresponding strategies. Thus, high-beta stocks tend to be large stocks and low-beta stocks tend to be value stocks. Finally, the momentum factor loading is insignificant for all strategies. These findings are in clear contrast to the findings for the S\&P 1500 universe.

The performance of the low-beta strategies for the smaller investible universe is driven mainly by sensitivities to common risk factors, such that none of the strategies is able to yield significant risk-adjusted returns, and the positive average returns of the strategies can be explained mainly by the risk factors. Nevertheless, even in this case, the results emphasize the importance of how an investment strategy actually tries to exploit the low-beta anomaly. As observed in the factor loadings, overweighting high-beta stocks leads to a substantial size exposure, and overweighting low-beta stocks results in an extensive value exposure. All of our results for both investible universes highlight that there is no low-beta strategy per se, but different strategies exist with quite different properties, which must be taken into account when designing a low-beta strategy.

\footnotetext{
${ }^{19}$ Blitz (2016) investigates the relation between the low-volatility effect and the value effect and concludes that the low-volatility effect is the more robust anomaly. It might therefore be misleading to "explain" the returns of a low-beta strategy by a value factor, because causality might well be the other way round.
} 


\subsection{Conclusion}

This paper addresses the issue that different choices exist to exploit the low-beta anomaly via trading strategies. Our empirical results show that whether a low-beta strategy puts more weight on buying low-beta stocks or on selling high-beta stocks can make a significant difference. Likewise, it is important how the investible universe is defined. Only in a larger stock universe, such as the S\&P 1500 Index, significant alphas can be achieved. Among a number of additional design elements, the estimation method of beta and the weightings within the low-beta portfolio and the high-beta portfolio have the greatest impact on riskreturn characteristics. For the basic approach the highest average return and risk-adjusted return is earned by the strategies overweighting low-beta stocks. For Frazzini/Pedersen and Dimson estimation, the level of average returns is higher and overweighting high-beta stocks produces the highest risk-adjusted returns. A similar pattern can be found regarding the weighting. Under equal weighting and beta weighting, overweighting of low-beta stocks achieves higher average returns and lower risk. Under value weighting, however, putting more weight on selling high-beta stocks leads to higher average returns. We also find that a shorter estimation window leads to higher average returns, and a yearly rebalancing of the portfolios is sufficient.

In the smaller stock universe, consisting of the stocks included in the S\&P 500 Index, we observe no significant alphas, but we emphasize that the return characteristics strongly depend on the design elements. Strategies that overweight low-beta stocks deliver higher average returns and are very sensitive to the value factor, whereas strategies that overweight high-beta stocks have no value exposure, but a higher size exposure. These results stress the importance of selecting a low-beta strategy that is in line with the desired portfolio characteristics and that does not take the investor or portfolio manager by surprise.

Our findings support the view that the low-beta anomaly is due to small, more illiquid stocks. A first reason is that significant risk-adjusted returns exist only for the larger investible universe. A second indication is that giving higher weights to small stocks within the low-beta portfolio (equal weighting instead of value weighting) improves performance. Moreover, when the basic estimation approach is used, risk-adjusted returns result primarily 
from a positive premium earned by low-beta stocks relative to the whole market and not from a negative premium of high-beta stocks. This is good news for investors who are reluctant to follow strategies requiring short positions, because the premium can very well be exploited by just buying low-beta stocks. 


\title{
3 Markowitz with Regret
}

\author{
with Rainer Baule and Olaf Korn \\ under Review at the Journal of Economic Dynamics and Control
}

\begin{abstract}
We provide a framework to integrate regret as an additional decision criterion in Markowitz's model of portfolio selection. Regret can be captured by adjusting the vector of expected returns or alternatively by adjusting the return covariance matrix, retaining the tractability of the Markowitz model. The regret model, however, has very different implications for how asset characteristics affect optimal portfolio weights. While the impact of the expected return and the skewness of an asset is strengthened, the impact of the variance shrinks. Portfolio weights might even increase with increasing variance. Moreover, we show for a variety of real portfolios that the effects of regret on optimal portfolio weights are large.
\end{abstract}




\subsection{Introduction}

The portfolio selection approach by Markowitz $(1952,1987)$ is a cornerstone of modern finance. It provides a formal yet tractable procedure to find optimal portfolios. This tractability comes at a cost, however. The focus on the mean and variance of returns neglects higher-order return moments like skewness and kurtosis. Moreover, it ignores any aspects beyond final wealth that can be important for investors, in particular emotional and self-expressive benefits. Harry Markowitz himself acknowledges the importance of such benefits. When asked about his personal pension savings in an interview (Zweig 1998) he admitted, "My intention was to minimize my future regret. So I split my contribution fifty-fifty between bonds and equities." ${ }^{1}$ Markowitz's statement raises interesting questions. How can regret be considered in portfolio selection without losing the formal elegance and tractability of Markowitz's approach? Within such a new framework, is it really the best choice to split the investment evenly between stocks and bonds? What do optimal portfolios look like and what makes an asset attractive if regret is considered in addition to final wealth?

Answers to these questions are important because they can help investors to find better portfolios by allowing for a more realistic preference specification while maintaining the transparency and tractability of Markowitz's approach. The contribution of this paper is to provide such answers. It proposes a framework of portfolio selection with regret and analyzes the properties of optimal portfolios. Regret is understood as an emotional feeling caused by the ex-post knowledge that a different decision — other than the one actually taken — would have led to a better outcome. Decision-making under regret aversion has been developed formally in the literature (Bell 1982, 1983; Loomes and Sugden 1982) and can rest on an axiomatic foundation (Sugden 1993; Diecidue and Somasundaram 2017). In our specific framework an investor potentially cares about two attributes of a portfolio: its return and the regret resulting from holding it. With respect to the trade-off between the expected benefits of a portfolio and its risk we retain a simple mean-variance

\footnotetext{
${ }^{1}$ This confession is only one example for the importance of regret in investment decisions. In a recent study, Frydman and Camerer (2016) show that neural measures of regret are related to the decision-making of participants in an experimental asset market.
} 
criterion, leaving the Markowitz approach as a special case of our model. As our analysis shows, incorporating regret into portfolio selection has interesting implications for the optimization problem itself and the composition of the resulting portfolios. With respect to the optimization problem, we show that it can be formulated in different ways, offering different advantages in terms of implementation and interpretation. One alternative leaves the vector of expected returns unchanged and incorporates regret effects by adjusting the covariance matrix only. In an alternative, a regret-adjustment of the expected returns is required while the covariance matrix can be left unchanged. Essentially, portfolio optimization in our regret framework is not more complicated than in the Markowitz world. However, we show that regret can change optimal portfolios considerably. It makes assets with higher expected returns and higher skewness more attractive than in the Markowitz model. In contrast, the impact of the return standard deviation is diminished. It is even possible that the portfolio weight of an asset increases with standard deviation. Using real world data for different investible universes, we demonstrate that such regret effects on optimal portfolio weights can be large.

Although our model is primarily meant to be a useful tool to find optimal portfolios for individual investment decisions, the model's main implications are well in line with observed market characteristics. For example, it is a persistent empirical finding that low volatility stocks tend to earn higher average returns than high volatility stocks (Ang et al. 2006, 2009), which seems to contradict the trade-off between risk and return. However, such a pattern can well be in line with the risk-return trade-off in the regret model. Similarly, in our regret model a positive skewness makes an asset more attractive, which is not captured by the Markowitz model but is in line with the empirical observation of skewness premiums (Conrad et al. 2013).

Our work belongs to the literature on alternative portfolio theories. As we show, portfolio optimization with regret can be implemented by replacing variance with a different measure of risk, the regret-adjusted variance. In this respect our approach is similar to portfolio models that use alternative risk measures, like lower partial moments (Harlow 1991; Zhu et al. 2009; Klebaner et al. 2017) or conditional value-at-risk (Quaranta and Zaffaroni 2008). Moreover, our work belongs to the literature on behavioral portfolio theories, studying 
portfolio problems with non-standard preferences, like loss aversion and disappointment aversion (Ang et al. 2005; Driessen and Maenhout 2007; Shi et al. 2015) or different mental accounts (Shefrin and Statman 2000; Das et al. 2010; Das and Statman 2013). However, there is a fundamental difference between these approaches and the regret model. In the regret model, utility does not depend on the final outcome of the chosen portfolio alone, but also on the outcome of other feasible portfolios that were not chosen. In this sense the regret model is related to benchmarking (with the ex-post best portfolio as the benchmark) and the analysis of tracking errors (with respect to the ex-post best portfolio). Stated differently, the regret approach does not simply change the view on the risk of an investor's final wealth but adds a distinctively different investor need to the portfolio problem.

To our knowledge only three other papers provide theoretical results on the effects of investor regret on portfolio choice. Muermann et al. (2006) study the asset allocation between a risk-free asset and a risky asset in defined contribution pension schemes. Michenaud and Solnik (2008) study the currency hedging decision of investment managers, a setting that is structurally equivalent to an asset allocation problem with one risk-free and one risky asset. $^{2}$ The two papers provide important insights that are in line with our findings. Most importantly, when investors consider regret they tend to "hedge away from the extremes". The works by Muermann et al. (2006) and Michenaud and Solnik (2008), however, are confined to a specific two-asset problem. In contrast, our analysis studies the Markowitz problem of $N$ risky assets and our results equally apply to the case with $N-1$ risky and one risk-free asset. As we show, a setting with multiple risky assets has a much richer structure. In particular, the dependence structure between assets changes the problem because it has an impact on the regret risk of a portfolio. Gollier and Salanié (2006) also allow for multiple assets in their framework of a complete-market Arrow-Debreu economy. They study optimal asset allocation as well as equilibrium asset prices when investors are sensitive to regret. Some insights from their model also show up in our results, in particular the importance of skewness for the attractiveness of an asset when investors are regret-averse. The work by Gollier and Salanié focuses on structural properties and

\footnotetext{
${ }^{2}$ In Michenaud's and Solnik's model there is also another stochastic component beyond exchange-rate risk: the return of the foreign asset in local currency. This second stochastic component cannot be influenced by the investor, however. Therefore, the investment problem is to choose between a risk-free and a risky asset with additional background risk.
} 
equilibrium implications. In contrast, the main goal of this paper is to provide a tractable extension of the Markowitz model which requires few inputs, is easy to understand for investors, and is relatively easy to implement. In particular, in our setting, parameter estimation can proceed in the same way as in the Markowitz model and we are able to obtain optimal portfolio weights in closed form if short-sales constraints are not binding, that is, if all weights are strictly positive.

The remainder of the paper is structured as follows: Section 3.2 introduces our setting and delivers some results on the structure of the portfolio problem and the properties of optimal portfolio weights. In the following Section 3.3, we present a deeper analysis of the impact of regret on portfolio weights and ask what distributional characteristics make an asset more or less attractive than in the Markowitz model. A comparative static analysis based on analytical results and simulations is presented. Section 3.4 demonstrates the quantitative importance of regret effects for real portfolios. Some conclusions are provided in Section 3.5.

\subsection{The Portfolio Problem}

We study the one-period portfolio problem where an investor can choose among a set of risky assets or funds. The investor considers two criteria to assess a portfolio. The first is return, as in classical portfolio selection, representing the benefits of wealth and consumption opportunities. The second is the avoidance of regret, representing the emotional and self-expressive benefits of being not too far from the ex-post best choice. To formalize this idea, we have to define the investment opportunity set and identify the ex-post best asset within this set. Assume that $N$ risky assets are available at the beginning of the period and denote the random return vector by $R:=\left(R_{1}, R_{2}, \ldots, R_{N}\right)^{t r}$. In our setting, the investment opportunity set consists of all combinations of these assets that are attainable under the budget constraint and fulfill some restrictions on leverage. Restrictions on leverage are required because the ex-post best strategy would otherwise lead to infinite wealth, which is certainly unrealistic and not a meaningful benchmark for an investor's regret. The reason is that unlimited leverage would allow an infinite amount of money to be held in 
the ex-post best-performing asset, financed via short selling the worst-performing asset. The imposition of leverage constraints is thus crucial for our approach. We see this as an advantage of the regret model rather than a drawback, because being forced to be explicit about an investor's leverage constraints adds realism to the model. For ease of exposition and because of their practical importance ${ }^{3}$, we impose short-sales constraints on all assets, that is, we do not allow for any leverage. However, the analysis can easily be adapted to incorporate any investor-specific restrictions. With short-sales constraints, the ex-post best strategy is to hold the full budget in the asset with the highest realized return. This ex-post best asset yields a return of $R_{\max }:=\max \left(R_{1}, R_{2}, \ldots, R_{N}\right)$. For any attainable portfolio $P$, regret is then defined as the difference between this benchmark return and the portfolio return $R_{P}$, that is., regret is the under-performance in percentage points compared to the ex-post best-performing portfolio that had been feasible ex ante. This definition of regret is not the standard one used in the literature, which usually defines regret as a difference in utility, not return. Because we work on an extension of the Markowitz model in this paper, our framework is mean-variance analysis instead of expected utility maximization, ${ }^{4}$ and we are looking for an appropriate regret measure for the mean-variance investor. From an ex-post (end-of-period) perspective an expected utility maximizer could well calculate and compare the utility levels resulting from different portfolios. However, variance risk will have disappeared at the end of the period, leaving the mean-variance investor with the known returns of the different alternatives. In our view it is therefore a natural choice to define regret as a return difference in the mean-variance decision framework.

A crucial issue is to specify the (relative) importance of the two criteria of return and regret for an investor. To express relative importance, we use the regret-adjusted return

\footnotetext{
${ }^{3}$ In practice, many investors prefer long-only portfolios over long-short portfolios. Moreover, it has been shown that short-sales constraints help to improve the out-of-sample performance of portfolio strategies (Frost and Savarino 1988; Jagannathan and Ma 2003).

${ }^{4}$ Although the mean-variance approach and the expected-utility approach are different concepts, the literature has shown various linkages. For example, Meyer (1987) and Bigelow (1993) provide conditions under which the two approaches lead to the same ranking of decision alternatives. Even if these conditions are not met, restricting choices to the set of mean-variance efficient portfolios often leads to minor utility losses for investors (Levy and Markowitz 1979; Kroll et al. 1984).
} 
$Z_{P}$ of a portfolio $P$, which is defined as

$$
\begin{aligned}
Z_{P} & :=(1-\alpha) R_{P}-\alpha \text { Regret } \\
& =(1-\alpha) R_{P}-\alpha\left(R_{\max }-R_{P}\right) \\
& =R_{P}-\alpha R_{\max }
\end{aligned}
$$

with $\alpha \in[0,1]$. The regret-adjusted return naturally combines the two criteria. It increases with higher portfolio returns and decreases with higher regret. The parameter $\alpha$ governs the relative importance of the two criteria, thereby reflecting the corresponding investor preferences. With $\alpha=0$ the investor does not care about regret and we are back at the classical portfolio problem. In the case of the other extreme $(\alpha=1)$, the investor only considers regret. We will call the former the Markowitz case and the latter the pure regret case.

Because the focus of our paper is to extend Markowitz's portfolio selection by incorporating regret as an additional criterion, we follow the classical approach and assume that investors evaluate portfolios based on the first two return moments, in our case referring to regretadjusted returns. In terms of the two components of $Z_{P}$, such preferences imply that investors like higher expected portfolio returns, dislike higher expected regret and dislike higher risks (variances) both with respect to portfolio returns and regret. ${ }^{5}$ Under such $\mu-\sigma$ preferences, the investor's optimization problem in the regret model is finally given by

$$
\begin{aligned}
\max _{\omega} \pi\left(Z_{P}\right)= & E\left(Z_{P}\right)-\gamma \operatorname{Var}\left(Z_{P}\right), \\
\text { s.t. } & \sum_{i=1}^{N} \omega_{i}=1, \\
& \omega_{i} \geq 0 \forall i,
\end{aligned}
$$

where $\omega:=\left(\omega_{1}, \omega_{2}, \ldots, \omega_{N}\right)^{t r}$ is the vector of portfolio weights so that $R_{P}=\omega^{t r} R$, and the parameter $\gamma>0$ is a measure of risk aversion.

\footnotetext{
${ }^{5}$ Bleichrodt et al. (2010) provide empirical evidence that decision makers indeed are averse to regret risk.
} 
Having set up the portfolio problem, we will discuss some structural properties of this problem which have important practical implications and help us to understand the effects of regret on optimal portfolios.

Property 1 (Adjustment of Covariance Matrix): Optimal portfolio weights can be found as in standard $\mu-\sigma$ optimization, using the (unadjusted) vector of expected returns, $\mu=E[R]$, and an adjusted covariance matrix that contains the variances and covariances of the regret-adjusted returns of the $N$ assets, $Z_{1}, \ldots, Z_{N}$.

To show that Property 1 holds true, let us write the investor's preference function from Equation 3.2 as a function of the portfolio weights $\omega$ :

$$
\pi\left(Z_{P}\right)=\omega^{t r} \mu_{r a}-\gamma\left(\omega^{t r} \Sigma_{r a} \omega\right)
$$

where $\mu_{r a}=\mu-\alpha E\left[R_{\text {max }}\right]$ is the column vector of expected regret-adjusted returns $Z_{1}, \ldots, Z_{N}$ and $\Sigma_{r a}$ is the corresponding covariance matrix. Because portfolio weights add up to one, we can rewrite Equation 3.3 as

$$
\pi\left(Z_{P}\right)=\omega^{t r} \mu-\alpha E\left[R_{\max }\right]-\gamma\left(\omega^{t r} \Sigma_{r a} \omega\right)
$$

The term $\alpha E\left[R_{\max }\right]$ is independent of $\omega$, that is, it cannot be changed by choosing a different portfolio. Thus, for the determination of optimal portfolio weights we can ignore this term and work with $\mu$ instead of $\mu_{r a}$.

Property 1 is good news for the portfolio model with regret, because in a sense it is not more complicated to work with than with standard $\mu-\sigma$ analysis. The difference is that the risk measure is changed, from the variance of returns to the variance of regret-adjusted returns. This property makes our approach similar in structure (not necessarily in outcome) to alternative portfolio choice models that replace variance with alternative risk measures, for example lower partial moments. Moreover, once the investor has specified the parameter $\alpha$, the covariance matrix of regret-adjusted returns can be estimated from historical data 
in the same way as the covariance matrix of returns. ${ }^{6}$ Economically, the simple structure of the optimization problem is somewhat surprising, because our analysis did not just propagate an alternative risk measure, but started from the idea of integrating two separate investor needs, namely wealth and avoidance of regret, into portfolio choice. Two aspects are important for understanding why the resulting structure is so simple. First, in $\mu-\sigma$ analysis expected returns affect portfolio weights only via return differences between assets, not via the levels of expected returns. If we recall that the expected regret of investing in a single asset is just the expected return difference between the ex-post maximum-return asset and that specific asset, it becomes clear that the vector of individual assets' expected regret contains the same information on return differences as the vector of expected returns. The reason is simply that regret is measured according to the same benchmark for all assets, the return of the ex-post maximum-return asset. Because expected regret contains redundant information with respect to the determination of portfolio weights, it disappears from the optimization problem. However, it would be equally correct to say that expected returns disappear from the optimization problem as long as information on expected regret is incorporated. One can therefore conclude that both expected returns and expected regret are considered, but simply lead to the same portfolio weights. Second, why is it possible that the two different investor needs can be combined in a simple adjustment of the covariance matrix? The reason is that both attributes are measured on an equal footing, namely in terms of asset returns. Measuring regret, for example, in terms of utility differences resulting from return differences would destroy this simple structure. However, even in our simple setting, we need two preference parameters. The first, $\gamma$, determines the relative importance of expected return and risk, and the second, $\alpha$, determines the relative importance of return risk in comparison to regret risk.

Property 2 (Adjustment of Expected Returns): Alternatively, optimal portfolio weights can be found by using an adjusted vector of expected returns and the (unadjusted) return covariance matrix of the original returns $R_{1}, \ldots, R_{N}$. The adjustment of expected returns is made by replacing $E\left[R_{i}\right]$ with $E\left[R_{i}\right]+2 \alpha \gamma \operatorname{Cov}\left(R_{i}, R_{\max }\right) \forall i=1, \ldots, N$.

\footnotetext{
${ }^{6}$ Methods to improve estimation, like shrinkage (Ledoit and Wolf 2004) or more general norm constraints (DeMiguel et al. 2009), can be used for the covariance matrix of regret-adjusted returns in the same way as for the return covariance matrix.
} 
To show Property 2, we start by noting that the $(i, j)$ entry of the covariance matrix of regret-adjusted returns is

$$
\begin{aligned}
\left(\Sigma_{r a}\right)_{i, j} & =\operatorname{Cov}\left(R_{i}-\alpha R_{\text {max }}, R_{j}-\alpha R_{\text {max }}\right) \\
& =\operatorname{Cov}\left(R_{i}, R_{j}\right)-\alpha \operatorname{Cov}\left(R_{i}, R_{\text {max }}\right)-\alpha \operatorname{Cov}\left(R_{j}, R_{\text {max }}\right)+\alpha^{2} \operatorname{Var}\left(R_{\text {max }}\right) .
\end{aligned}
$$

It follows: ${ }^{7}$

$$
\begin{aligned}
\omega^{t r} \Sigma_{r a} \omega & =\omega^{t r} \Sigma \omega-2 \alpha \sum_{i} \omega_{i} \sum_{j} \omega_{j} \operatorname{Cov}\left(R_{j}, R_{\max }\right)+\alpha^{2} \operatorname{Var}\left(R_{\max }\right) \\
& =\omega^{t r} \Sigma \omega-2 \alpha \omega^{\operatorname{tr}} \operatorname{Cov}\left(R, R_{\max }\right)+\alpha^{2} \operatorname{Var}\left(R_{\max }\right) .
\end{aligned}
$$

Using the alternative expression for $\omega^{t r} \Sigma_{r a} \omega$ from the right hand side of Equation 3.6 and inserting it into Equation 3.4 yields

$$
\pi\left(Z_{P}\right)=\omega^{t r}\left(\mu+2 \alpha \gamma \operatorname{Cov}\left(R, R_{\max }\right)\right)-\gamma\left(\omega^{\operatorname{tr}} \Sigma \omega\right)-c
$$

with a constant $c=\alpha E\left[R_{\max }\right]+\gamma \alpha^{2} \operatorname{Var}\left(R_{\max }\right)$. This constant, however, is irrelevant for the determination of the optimal portfolio weights.

Property 2 provides a second representation of the optimization problem, which is in a sense the mirror image of the representation from Property 1. Instead of adjusting the covariances and leaving the expected returns unadjusted, we can alternatively use the unadjusted return covariance matrix and adjust the vector of expected returns. The adjustment that is made for each asset is very intuitive. A first observation is that we require both preference parameters $\alpha$ and $\gamma$ to be positive for any adjustment to appear. It is obvious that $\alpha=0$ leads to no adjustment, because we would then be back in the Markowitz case. But even with a positive $\alpha, \gamma=0$ would seemingly leave us without any regret effect. The reason was already discussed in the context of Property 1. If there is no risk aversion and only expected returns count, using either expected returns or expected regret leads to the same result, namely to the choice of the asset with the highest expected return, which has also

\footnotetext{
${ }^{7}$ The term $\operatorname{Cov}\left(R, R_{\max }\right)$ denotes a vector with covariances $\operatorname{Cov}\left(R_{j}, R_{\max }\right)$ for $j=1, \ldots, n$.
} 
the lowest expected regret. What the adjustment does, though, is to account for the effect of regret risk. The regret risk of an asset is expressed via the covariance between the asset's return and the return of the ex-post best asset. If this covariance is high, the returns of the asset and the benchmark move together and regret risk is low. Therefore, the asset receives a high positive return adjustment. However, again, what matters for the optimal portfolio weights are the differences between the assets' expected returns, not the levels. Thus, if the adjustments in expected returns were to be the same for all assets, there would be no effect on portfolio weights and it is crucial to look at the differences between the adjustments for different assets. What is particularly nice about the expected return adjustment is that the effects of regret risk on portfolio composition can be captured by only a single indicator per asset, which has moreover a simple economic interpretation. Deviations from average adjustments tell us what return premium or discount would make an asset in the regret model as attractive as in the Markowitz model. Looking at such numbers might also help investors to quantify their degree of regret-risk aversion. We will also use them in Section 3.3 to better understand the relative attractiveness of assets in the regret world as compared to the Markowitz world.

Property 3 (Weighted Average of Weights): If short-sales constraints are not binding, optimal portfolio weights in the regret model are the weighted average of optimal weights from the Markowitz case and the pure regret case, with relative shares being $(1-\alpha)$ and $\alpha$, respectively.

To show Property 3, note that according to Property 2 we can use a vector of adjusted expected returns and the unadjusted return covariance matrix to find optimal portfolio weights. Denote the vector of adjusted expected returns by

$$
\mu_{a d j}:=\mu+2 \alpha \gamma \operatorname{Cov}\left(R, R_{\max }\right)
$$


When short-sales constraints are not binding, the following closed-form solution for the optimal weights is available: ${ }^{8}$

$$
\omega_{r a}^{*}=\frac{1}{2 \gamma} \Sigma^{-1}\left(\mu_{a d j}-\frac{\underline{1}^{t r} \Sigma^{-1} \mu_{a d j}-2 \gamma}{\underline{1}^{t r} \Sigma^{-1} \underline{1}} \underline{1}\right),
$$

where 1 denotes a column vector of ones. We can now verify directly that a weighted average of the solutions for the Markowitz case (with weight $1-\alpha$ ) and the pure regret case (with weight $\alpha$ ) delivers the optimal solution in Equation 3.9. To see this note that we can concentrate on $\mu_{a d j}$, because no other terms depend on whether we are in the Markowitz case or in the pure regret case. However, it holds that

$$
\mu_{a d j}=(1-\alpha) \mu+\alpha\left[\mu+2 \gamma \operatorname{Cov}\left(R, R_{\max }\right)\right],
$$

which delivers the required result.

Property 3 provides a considerable simplification for the understanding of regret effects on portfolio composition. If short-sales constraints are not binding, an analysis of such effects can concentrate on just the differences between the Markowitz case and the pure regret case, because all other cases are simple combinations of these two. Even with binding short-sales constraints, where the result does not necessarily hold exactly, these two cases are important reference points.

In summary, Properties 1 to 3 provide important information for implementing the regret model and understanding the effects of regret. First, the specification of the expected return component is as simple (or better to say as difficult) as in the Markowitz approach, because there is no need to estimate expected regret. Second, regret-risk effects can be incorporated in two different ways, either by using the full covariance matrix of regret-adjusted returns or by using the covariances between the individual assets' returns and the ex-post best performing asset. All required quantities can be estimated from historical data as in the Markowitz case. The only additional requirement is the specification of the parameter $\alpha$.

\footnotetext{
${ }^{8} \mathrm{~A}$ derivation is provided in the Appendix 3.A.1.
} 
Third, the analysis of the pure regret case is often sufficient for an understanding of how the regret model changes the portfolio composition compared to the Markowitz model.

\subsection{Optimal Portfolios}

In this section we investigate in some detail how regret affects portfolio composition. In particular, we ask which characteristics of an asset make it more or less attractive than in the Markowitz world. ${ }^{9}$ The previous section already presented two properties that are very useful in this respect. First, it is regret risk, not expected regret, that leads to changes in portfolio composition as opposed to the Markowitz model. Second, an asset's covariance with the ex-post best asset is crucial for its attractiveness. These two properties can guide us in our understanding of optimal portfolios. Moreover, the second property stresses a fundamental characteristic of the regret model: Changes in the distribution of any individual asset usually change the distribution of the ex-post best asset - the common benchmark - and thereby change the regret risk of all other assets too.

\subsubsection{Two-asset Case}

A natural starting point for an analysis of the effects of asset characteristics on portfolio composition is the case of an investor choosing between two assets (asset classes, funds). As our base scenario, we consider two homogeneous assets, that is, assets with the same distribution. With homogeneous assets the optimal policy is to invest half of the budget in each asset, both in the Markowitz case and the pure regret case, which defines our reference point with respect to portfolio composition. The concrete specification of the base scenario uses a bivariate normal distribution with expected returns of $10 \%$, standard deviations of $25 \%$, and a correlation of $0.5 .{ }^{10}$ The risk-aversion parameter is $\gamma=3$. Since normally distributed returns provide an ideal setting for the Markowitz model, this assumption

\footnotetext{
${ }^{9}$ For the Markowitz model the effects of different input parameters on portfolio characteristics are well documented in the literature (Best and Grauer 1991; Chopra and Ziemba 1993). We can therefore concentrate on the differences between the Markowitz model and the regret model.

${ }^{10}$ The level of expected returns is actually irrelevant for the resulting portfolio weights. What matters is the difference between the expected returns of different assets.
} 
allows us to identify regret effects without being hampered by other limitations of the model. In the comparative static analyses to follow, we calculate the vector of mean returns and the covariance matrix of (regret-adjusted) returns for each parameter combination. With these inputs, we obtain optimal portfolio weights.

\subsubsection{Expected Return}

The effects of changing the expected return of one asset (Asset 1) are presented in Figure 3.1. The upper part of the figure (Part A) shows the optimal portfolio weights of Asset 1 for two cases. The first one is the Markowitz case without any regret effects $(\alpha=0)$. The second one is the pure regret case $(\alpha=1)$. We can restrict our attention to these two extreme cases because for any $\alpha$ between zero and one the resulting portfolio weights are just the weighted average weights according to Property 3. In both cases, the portfolio weight of Asset 1 increases with higher expected returns. However, the effect is stronger in the pure regret case, which implies that considering regret as an additional criterion in the Markowitz model will lead to an even higher sensitivity of portfolio weights with respect to expected returns. The lower part of the figure (Part B) provides an intuition for this result. It shows the return covariance of both assets with the ex-post best asset. According to Property 2, this quantity is crucial for the optimal portfolio weight. The covariance of Asset 1 increases with its expected return, reflecting the fact that a higher expected return makes it more likely to become the ex-post best asset. Hence the regret risk of Asset 1 shrinks. Importantly, changes in the expected return of Asset 1 also affect Asset 2, which can be seen from its decreasing return covariance with the ex-post best asset. The covariance decreases because Asset 2 is less likely to be the ex-post best asset when the expected return of Asset 1 grows. In summary, the reason why expected returns are more important in the regret model than in the Markowitz model is their impact on regret risk. A higher expected return of an asset makes it less risky with respect to regret and at the same time raises the regret risk of the other asset. 
Figure 3.1: Effects of a Shift in Expected Return

\section{Part A: Portfolio Weight of Asset 1}

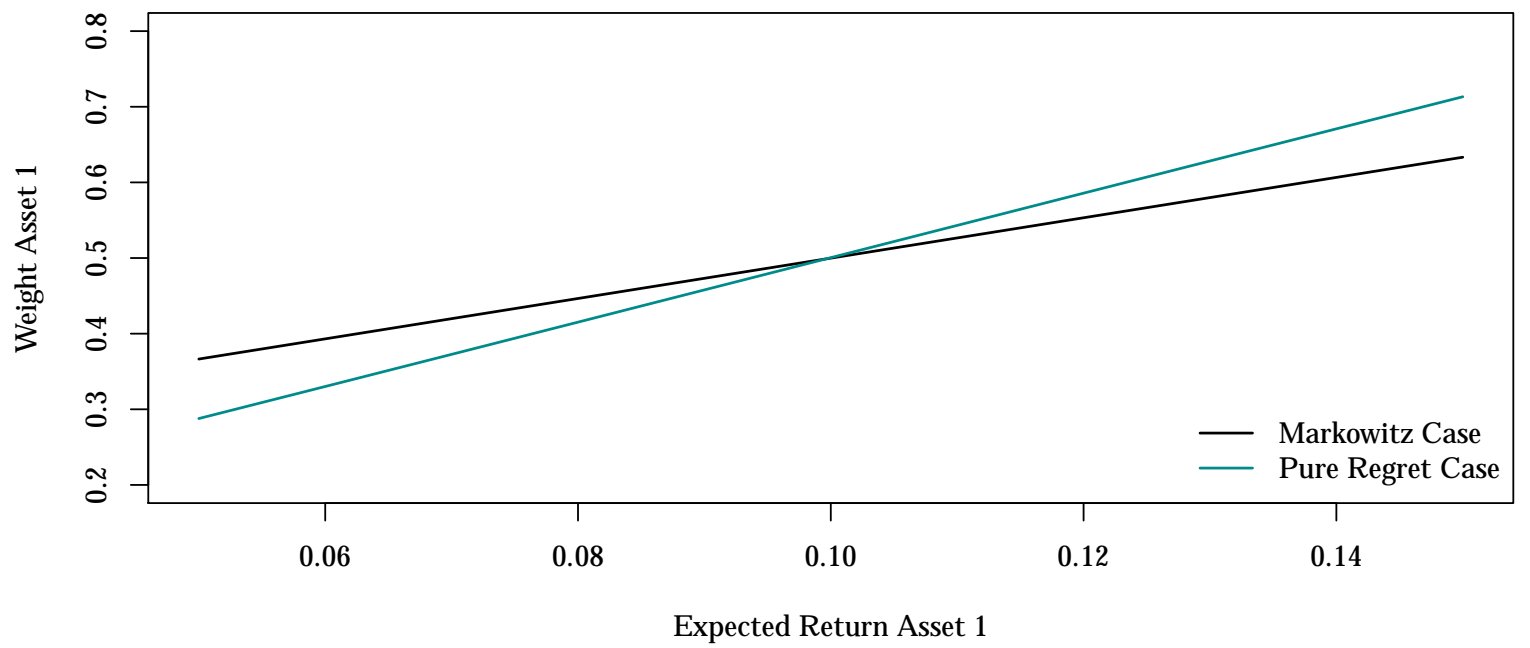

Part B: Covariances With Ex-post Best Asset

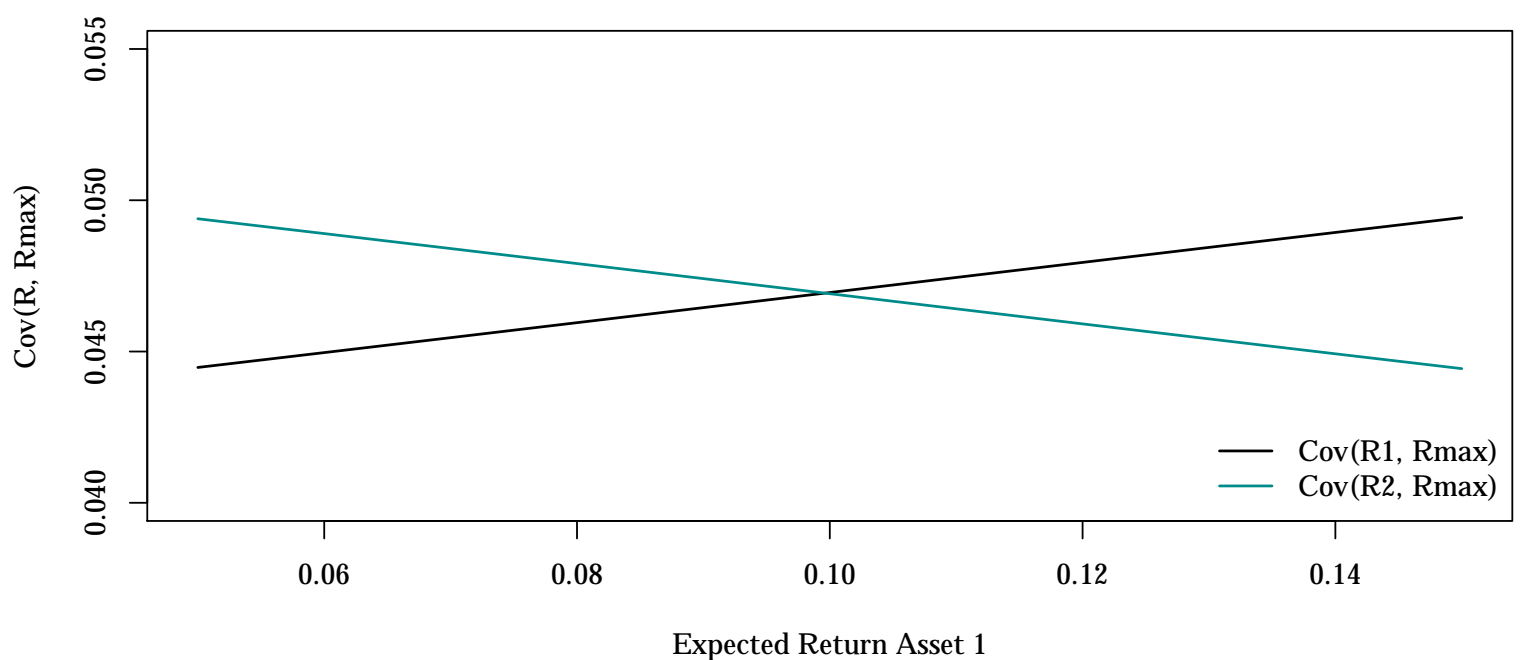

Note: Figure 3.1 shows the effects of a shift in the expected return of an asset (Asset 1) on its optimal portfolio weight (Part A) and on the covariance with the ex-post best asset (Part B). In Part A the black line refers to the Markowitz case $(\alpha=0)$ and the cyan line to the pure regret case $(\alpha=1)$. In Part B the black line refers to the return covariance of Asset 1 with the ex-post best asset $\left(\operatorname{Cov}\left(R_{1}, R_{\max }\right)\right)$ and the cyan line to the covariance of Asset 2 with the ex-post best asset $\left(\operatorname{Cov}\left(R_{2}, R_{\max }\right)\right)$. The base scenario uses two assets with a bivariate normal return distribution, identical expected returns of $10 \%$, identical standard deviations of $25 \%$, and a correlation of 0.5 . 


\subsubsection{Standard Deviation}

Figure 3.2 presents the effects of a change in the standard deviation of Asset 1. Again, Part A of the figure depicts the asset's portfolio weight. The Markowitz case and the pure regret case show very different reactions. Whereas the weight of Asset 1 strongly decreases with growing standard deviation in the Markowitz case, it does not change at all in the pure regret case. This finding implies that differences in standard deviation between the two assets do not translate into similar differences in regret risk. Actually, irrespective of the standard deviation of Asset 1, the relation between the two assets is completely symmetric with respect to regret. The distribution of regret-adjusted returns is always identical for both assets, given that the normal distribution is a symmetric distribution and mean returns are the same. Therefore, the two assets always have an identical standard deviation of regret-adjusted returns, regardless of any differences in (unadjusted) return standard deviations. Interestingly, the result that each asset obtains a portfolio weight of 0.5 in the pure regret case still holds if the standard deviation of Asset 1 drops to zero, making it a risk-free asset in the sense that returns are certain. However, in terms of regret risk this "risk-free asset" is still as risky as Asset 2. Part B of the figure shows the return covariances with the ex-post best asset. As we can see, these covariances increase for both assets. The reason is that the variance of the ex-post best asset grows if the standard deviation of one of the assets becomes larger. However, the covariance increases much more for Asset 1, finally leaving portfolio weights unaffected. 
Figure 3.2: Effects of a Shift in Standard Deviation

Part A: Portfolio Weight of Asset 1

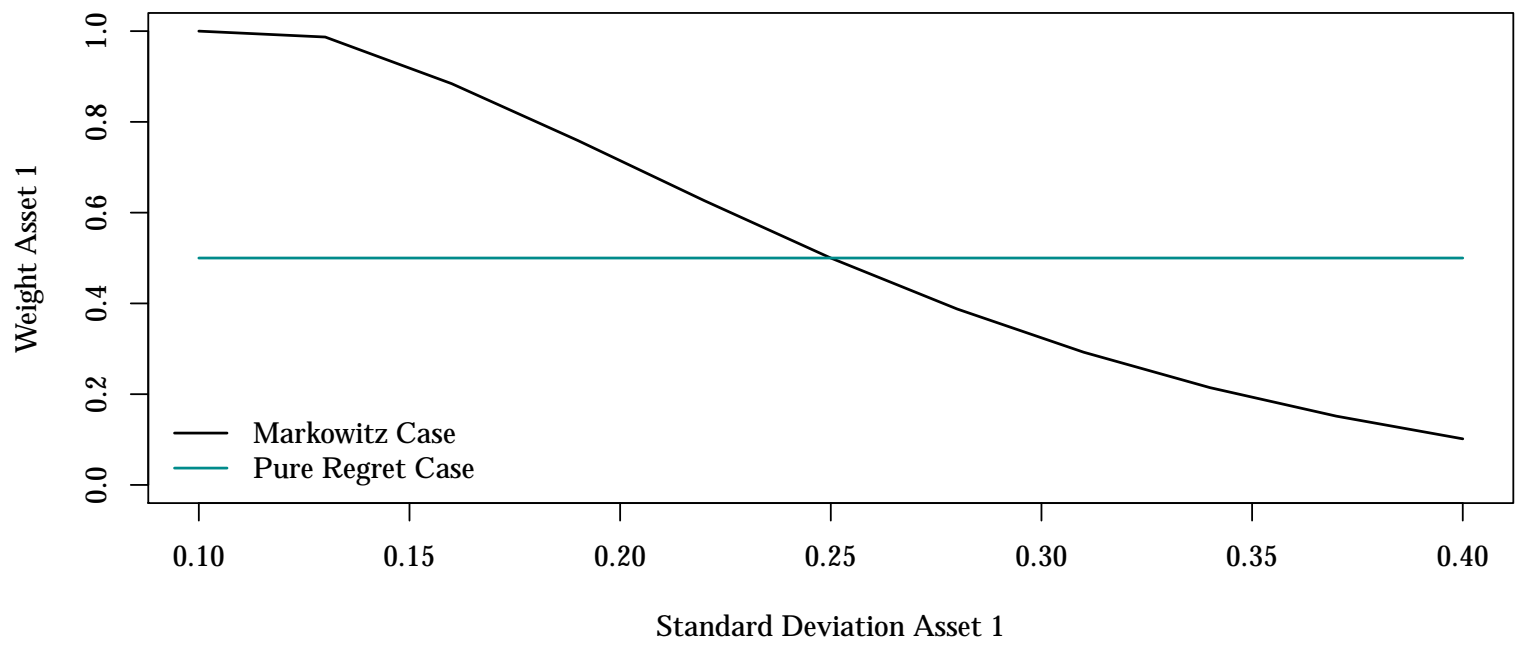

Part B: Covariances with Ex-post Best Asset

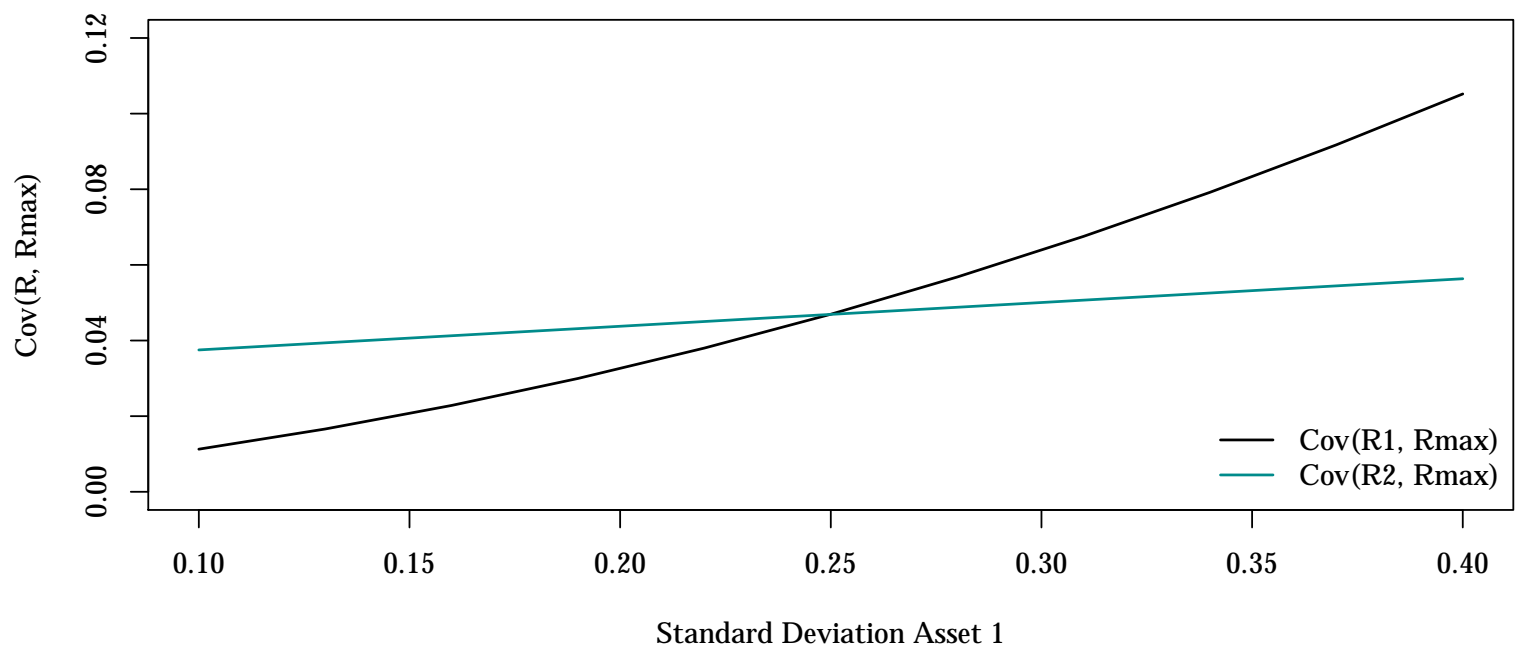

Note: Figure 3.2 shows the effects of a shift in the standard deviation of an asset (Asset 1) on its optimal portfolio weight (Part A) and on the covariance with the ex-post best asset (Part B). In Part A the black line refers to the Markowitz case $(\alpha=0)$ and the cyan line to the pure regret case $(\alpha=1)$. In Part B the black line refers to the return covariance of Asset 1 with the ex-post best asset $\left(\operatorname{Cov}\left(R_{1}, R_{\max }\right)\right)$ and the cyan line to the covariance of Asset 2 with the ex-post best asset $\left(\operatorname{Cov}\left(R_{1}, R_{\max }\right)\right)$. The base scenario uses two assets with a bivariate normal return distribution, identical expected returns of $10 \%$, identical standard deviations of $25 \%$, and a correlation of 0.5 . 
It is not generally the case, however, that changes in standard deviations do not influence the relative regret risk of the two assets, because the standard deviation effect interacts with expected returns. Figure 3.3 shows how the portfolio weight of Asset 1 change with its standard deviation when the expected returns of the two assets are different. Part A of the figure refers to a setting where the expected return of Asset 1 is lower than the one of Asset $2\left(\mu_{1}=0.05, \mu_{2}=0.1\right)$. In the setting of Part B it is the other way around $\left(\mu_{1}=0.15, \mu_{2}=0.1\right)$. For the pure regret case the effects of a change in standard deviation are substantially different in the two settings. When the expected return of Asset 1 is lower, its portfolio weight increases with higher return standard deviation. When Asset 1 is the asset with the higher expected return, however, a higher standard deviation reduces its portfolio weight. The reason for the different reactions is that the standard deviation affects the probability of becoming the ex-post best asset if expected returns are different. If an asset has a disadvantage in terms of expected returns, a higher standard deviation raises this probability and reduces its relative regret risk. In contrast, if an asset has an advantage in terms of higher expected returns, the probability of it becoming the ex-post best asset shrinks with growing standard deviation and leads to a higher relative regret risk. Therefore, Part A of the figure shows increasing portfolio weights, whereas Part B shows decreasing portfolio weights in the pure regret case. Irrespective of the relative magnitude of expected returns, the effects of a change in standard deviation in the pure regret case are still very different from the ones in the Markowitz case. Two aspects are most important. First, the sensitivities of portfolio weights with respect to changes in standard deviation are much smaller in absolute terms for the pure regret case. Second, if the standard deviation grows larger and larger in the pure regret case, the portfolio moves towards equal weights. In the Markowitz case, however, the portfolio will consist of a single asset. 


\section{Figure 3.3: Effects of a Shift in Standard Deviation with Different Expected Returns}

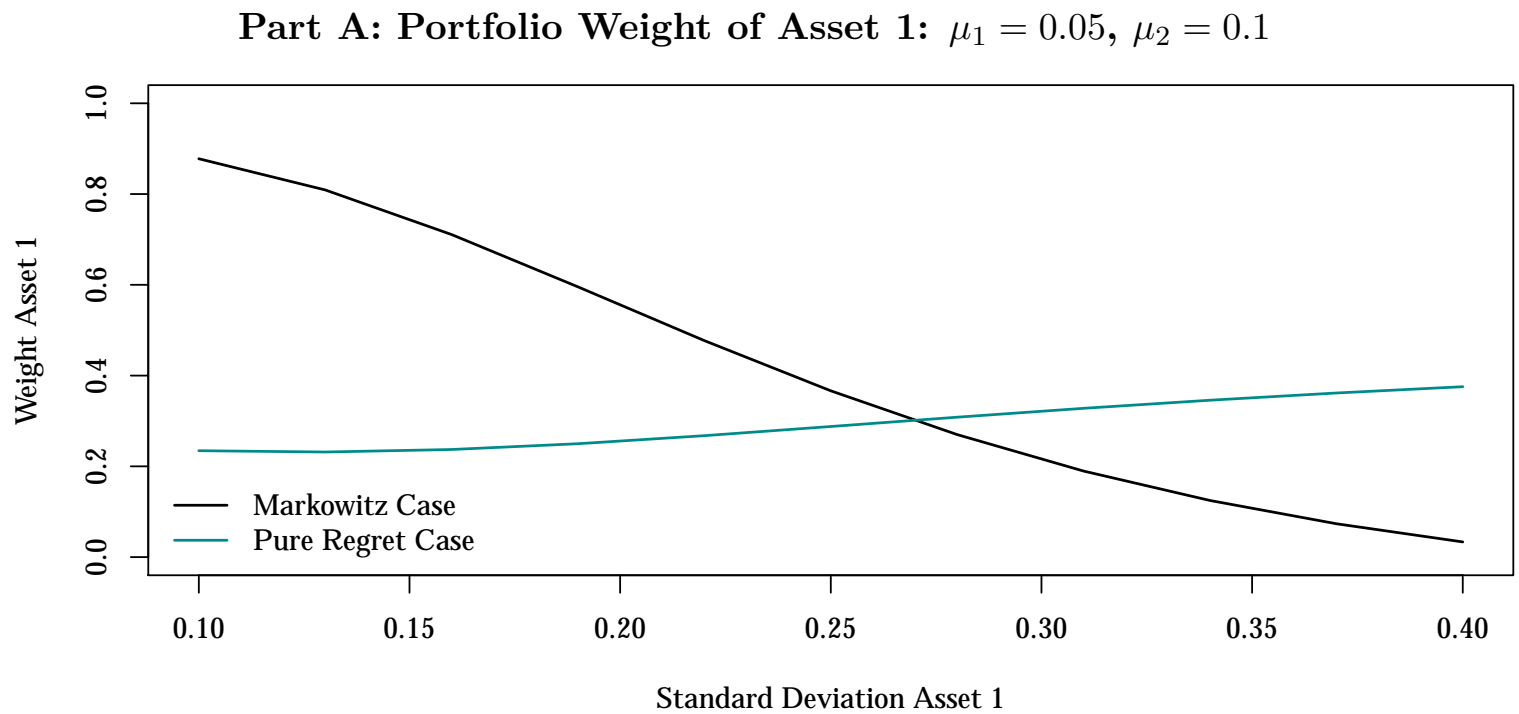

Part B: Portfolio Weight of Asset 1: $\mu_{1}=0.15, \mu_{2}=0.1$

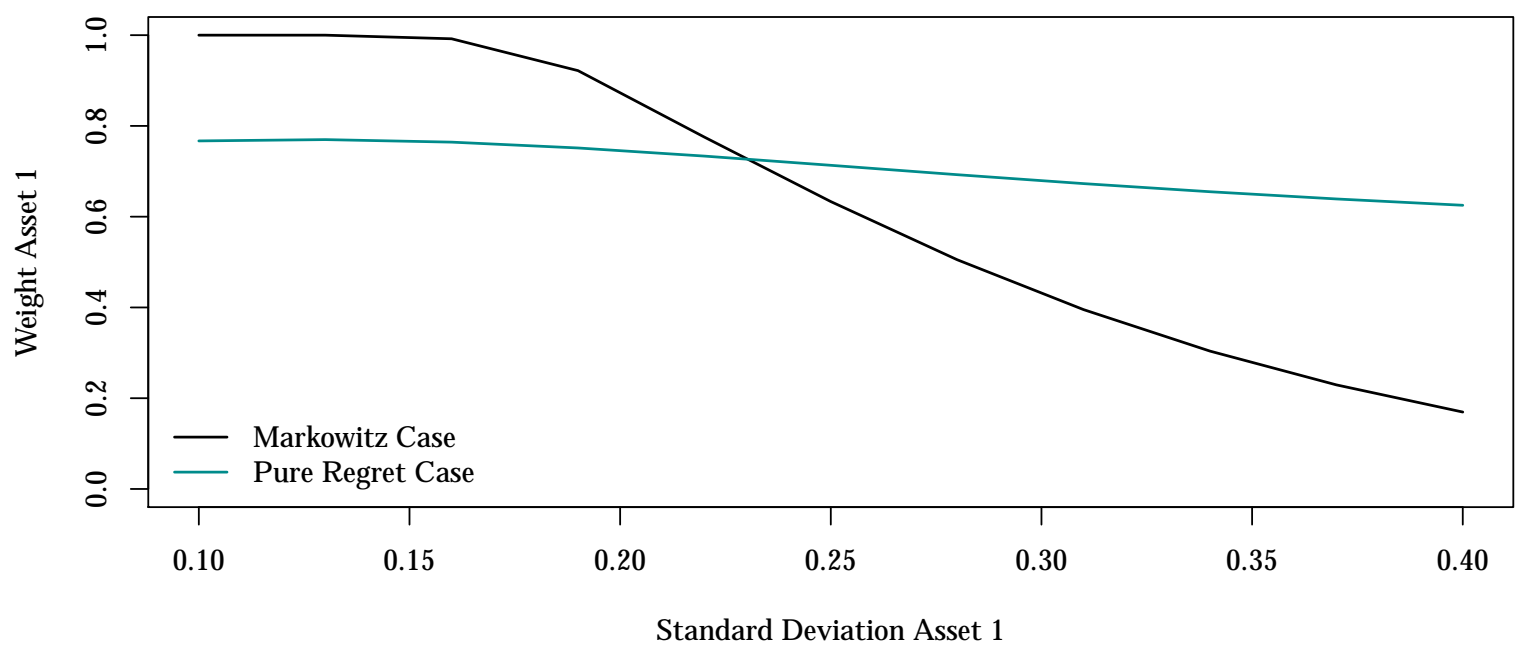

Note: Figure 3.3 shows the effects of a shift in the standard deviation of an asset (Asset 1) on its optimal portfolio weight in two different base scenarios with different expected returns of the assets. Both base scenario use two assets with a bivariate normal return distribution, identical standard deviations of $25 \%$, and a correlation of 0.5. The expected return of Asset 1 equals $5 \%$ and the expected return of Asset 2 equals $10 \%$ in the first base scenario. The effects of a standard deviation shift of Asset 1 on its optimal portfolio weight under this base scenario are depicted in Part A of the figure. In the second base scenario the expected return of Asset 1 equals $15 \%$ and the one of Asset 2 equals $10 \%$. The effects of a standard deviation shift of Asset 1 under this second base scenario are shown in Part B. The black lines refer to the Markowitz case $(\alpha=0)$ and the cyan lines to the pure regret case $(\alpha=1)$. 


\subsubsection{Skewness}

We have already seen that the expected return of an asset changes its risk in the regret world. Given the intuition behind this result, that is, the impact of expected returns on the probability of it becoming the ex-post best asset and on the risk of it deviating from this benchmark, it is plausible that the degree of asymmetry of the return distribution has also an effect on regret risk. We now examine this issue and ask to what extent optimal portfolio weights can be affected by the asymmetry of the return distribution in our model of portfolio choice. For such an analysis we have to drop the assumption of normally distributed returns. Instead, we use the Gram-Charlier density, which allows for a direct modeling of skewness. ${ }^{11}$ The Gram-Charlier density can be seen as an extension of the normal distribution with different higher moments. If skewness and excess kurtosis are zero, the density coincides with the normal density.

Our comparative static analysis leaves the setting of the base case untouched, but varies the skewness of Asset 1 between -1 and +1 . Part A of Figure 3.4 shows the corresponding effects. As expected, the portfolio weight increases with skewness in the pure regret case. For left-skewed distributions the portfolio weights are below 0.5 and for right-skewed distributions they are above 0.5. For highly asymmetric distributions, the quantitative effects are quite substantial, leading to weights of only about 0.35 for negative skewness and about 0.65 for positive skewness. Part B of the figure stresses the intuition behind the skewness effects. The higher the skewness of Asset 1 is, the higher the covariance with the ex-post best asset and the higher the relative advantage will be as compared to Asset 2 . In conclusion, a positive skewness is beneficial in the regret model because of its impact on the covariance matrix of regret-adjusted returns, making an asset with a positively skewed return distribution less risky than an asset with a negatively skewed distribution. This property is in sharp contrast to the Markowitz case, where skewness has no effect on portfolio weights.

\footnotetext{
${ }^{11}$ See, for example, Jondeau and Rockinger (2001).
} 
Figure 3.4: Effects of a Shift in Skewness

Part A: Portfolio Weight of Asset 1

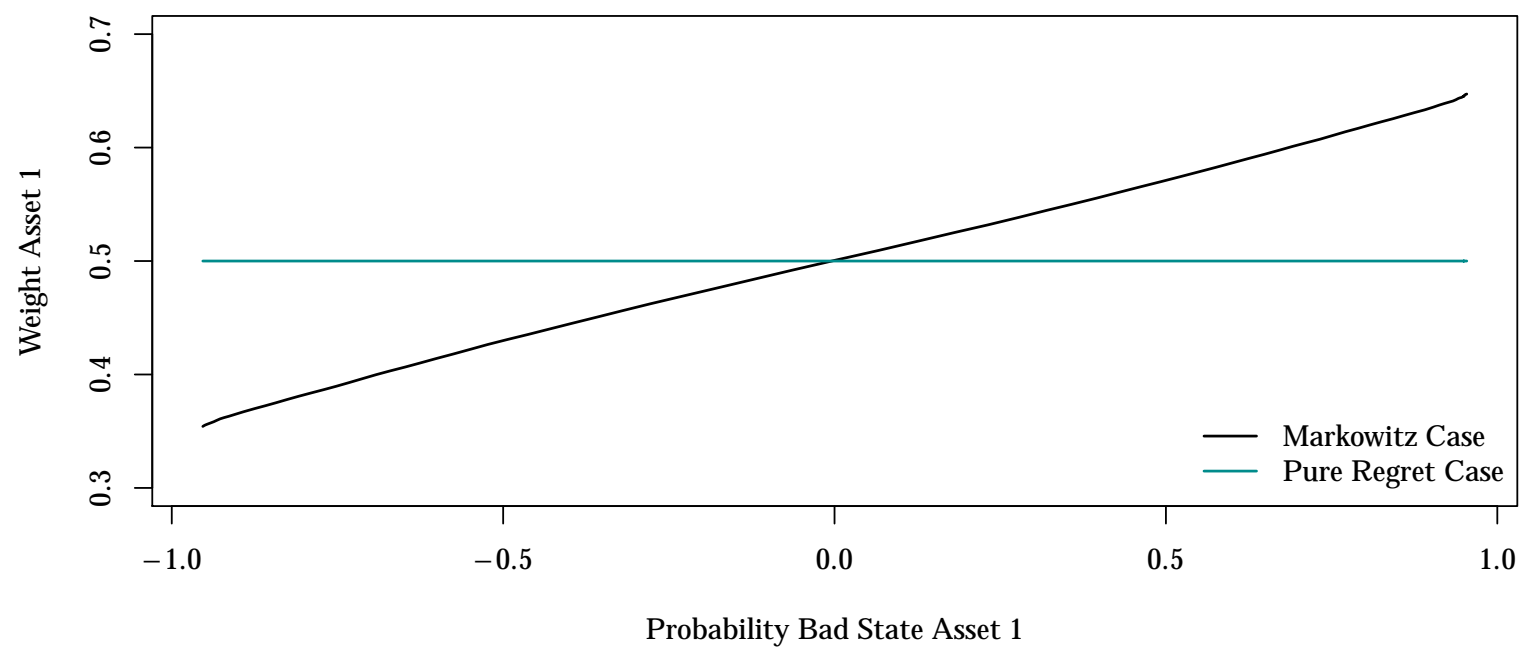

Part B: Covariances with Ex-post Best Asset

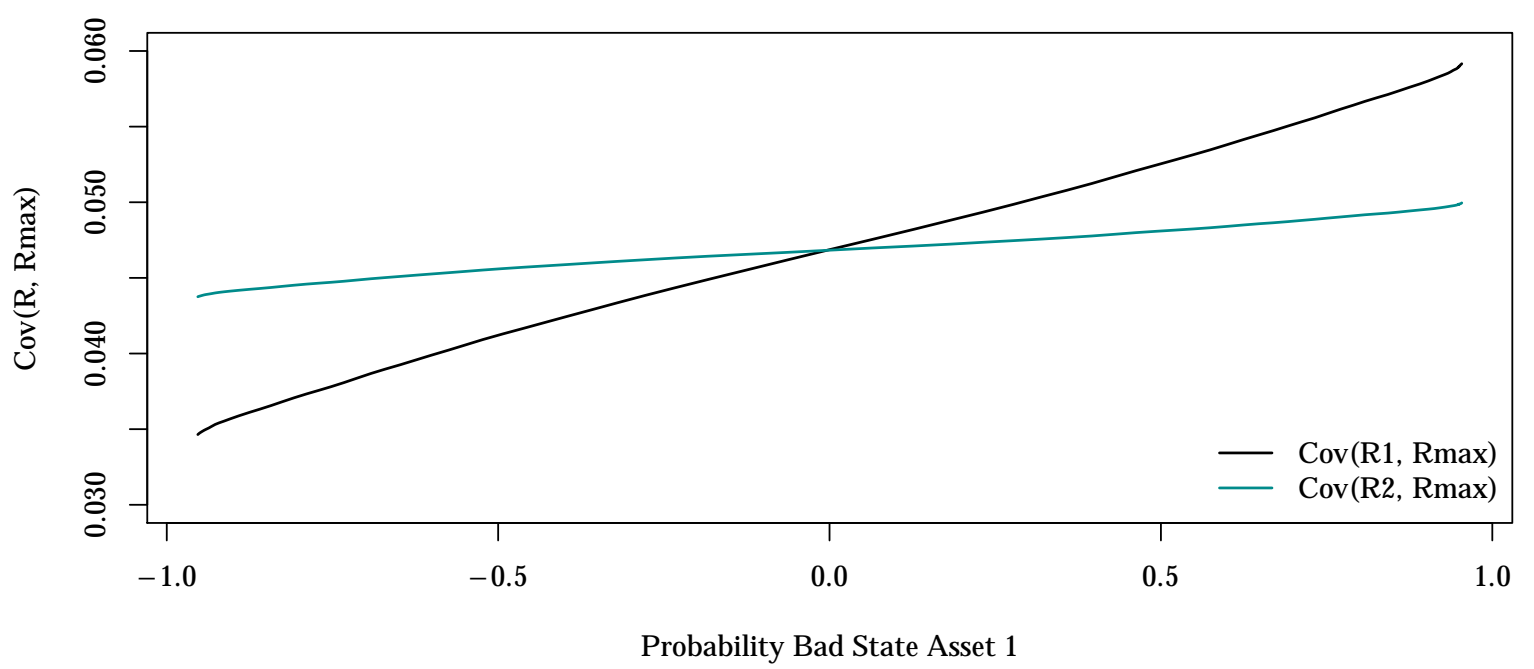

Note: Figure 3.4 shows the effects of a a changing return skewness of an asset (Asset 1) on its optimal portfolio weight (Part A) and on the covariance with the ex-post best asset (Part B). In Part A the black line refers to the Markowitz case $(\alpha=0)$ and the cyan line to the pure regret case $(\alpha=1)$. In Part B the black line refers to the return covariance of Asset 1 with the ex-post best asset $\left(\operatorname{Cov}\left(R_{1}, R_{\max }\right)\right)$ and the cyan line the covariance of Asset 2 with the ex-post best asset $\left(\operatorname{Cov}\left(R_{2}, R_{\max }\right)\right)$. The base scenario uses two assets with a bivariate normal return distribution, identical expected returns of $10 \%$, identical standard deviations of $25 \%$, and a correlation of 0.5 . For Asset 1 a Gram-Charlier density with varying skewness is assumed, while the return distribution of Asset 2 stays normal with zero skewness. 


\subsubsection{Summary}

Our analysis of the effects of different return moments on optimal portfolio weights has highlighted some important differences between the regret model and the classical Markowitz model. Both the expected return and the return skewness of an asset have an impact on the standard deviation of the asset's regret-adjusted return and the corresponding regretadjusted return standard deviation of the other asset. The higher these odd moments are, the lower an asset's relative regret risk will be. In contrast, these moments have no effect on the (unadjusted) return standard deviation of both assets. As a consequence, first and third moments influence portfolio weights more strongly in the regret model than in the Markowitz model. A higher return standard deviation of an asset, however, does not translate directly into a higher relative regret risk. It may even reduce relative regret risk, as we have seen from the case where the asset has a lower expected return than the other asset. What do these findings mean for the concentration or diversification of portfolios? Does the regret model lead to more or less diversified portfolios than the Markowitz approach? The answer to this question depends on the kind of heterogeneity of the investible universe. If the assets are very heterogeneous with respect to either first or third moments but quite homogenous with respect to second moments, the resulting optimal portfolio will tend to be more concentrated under the regret model than under the Markowitz model. If the assets are quite homogeneous with respect to first and third moments but quite different with respect to standard deviation, it will be the other way around, that is, the regret model will lead to less concentrated portfolios.

\subsubsection{Multi-asset Case}

The two-asset case provides important insights regarding the impact of regret on optimal portfolios. However, it does not capture the full richness of potential effects. We therefore look at the multi-asset case now and ask two questions: Do the general effects hold true regardless of the number of assets? What kind of structure is tied to two assets without carrying over to the case of more than two assets? 


\subsubsection{Expected Return}

A first answer to these questions can be given for the impact of expected returns on portfolio weights. As is apparent from Figure 3.1, taking regret into consideration leads to a higher sensitivity of optimal portfolio weights with respect to expected returns than the classical Markowitz model. This result also holds generally for $N$ assets. As long as short-sales constraints are not binding, the expected return sensitivity will always be positive in the regret model and larger than in the Markowitz model. A proof is given in the Appendix 3.A.2. We show that the result is not restricted to the case of a multivariate normal distribution but holds for any elliptical distribution. Intuitively, a higher expected return of an asset unambiguously reduces its regret risk and therefore makes it relatively more attractive than in the Markowitz model, regardless of the number of assets in the investible universe.

\subsubsection{Standard Deviation}

With respect to a comparative-static change in the standard deviation of an asset, the two-asset case is somehow special. As we have seen in Figure 3.2, under a bivariate normal distribution and identical expected returns there is no effect of the standard deviation at all in the pure regret case. Figure 3.5 shows the analogous situation for three assets (Part A) and ten assets (Part B), respectively. The plots are again based on multivariate normal distributions with identical means of $10 \%$, identical standard deviations of $25 \%$, and identical correlations of 0.5 between all assets as the base scenario. In this base scenario of homogenous assets the optimal portfolios weights equal $1 / \mathrm{N}$ for all assets, both in the Markowitz case and in the pure regret case. As Figure 3.5 shows, increasing the standard deviation of one asset (Asset 1) actually increases the optimal portfolio weight of this asset in the pure regret case, that is, a higher standard deviation lowers the regret risk of the asset relative to the regret risk of the other assets. The reason is that in the multi-asset case, the standard deviation has an impact on the asset's probability of being the ex-post best one even if the expected returns of all assets are identical. To point out the idea, consider an asset with no return risk, that is, a certain return of $10 \%$. If there is 
only one other risky asset with an expected return of $10 \%$, the probability of it being the ex-post best asset will be 0.5 , regardless of the standard deviation of this other asset. If there are several risky assets, however, the probability of it being the ex-post best asset can deviate from 1/N. For example, with two uncorrelated risky assets and one risk-free asset, the probability that the risk-free asset will be the ex-post best asset is only $1 / 4$ (the probability that both risky assets have a return below 10\%) and not $1 / 3$, which would be the case with three homogeneous assets. This argument, however, suggests that a changing standard deviation can also work in the opposite direction, because the standard deviation effect interacts with the correlation in the multi-asset setting. If the return correlation between the two risky assets approaches one, the probability that the risk-free asset is the ex-post best asset approaches $1 / 2$, which is above the value of $1 / 3$ that is obtained in the homogeneous asset case. For example, if we repeat the simulation from Part A of Figure 3.5 using a correlation of 0.95 instead of a correlation of 0.5 , the optimal portfolio weight of Asset 1 indeed drops from a value of about 0.41 for a standard deviation of $10 \%$ to a value of $1 / 3$ for the base scenario standard deviation of $25 \%$. All these results show that a shift in the standard deviation can produce different effects on optimal portfolio weights in the regret model if multiple assets are considered and the simple message from Figure 3.2 does not carry over to the multi-asset case. What does carry over, however, is the economic intuition behind the results. If a comparative-static shift of a parameter changes the probability of an asset to become the ex-post best asset, it is most likely that this shift will also affect optimal portfolio weights. 
Figure 3.5: Effects of a Shift in Standard Deviation with Multiple Assets

Part A: Portfolio Weight of Asset 1: Three Assets

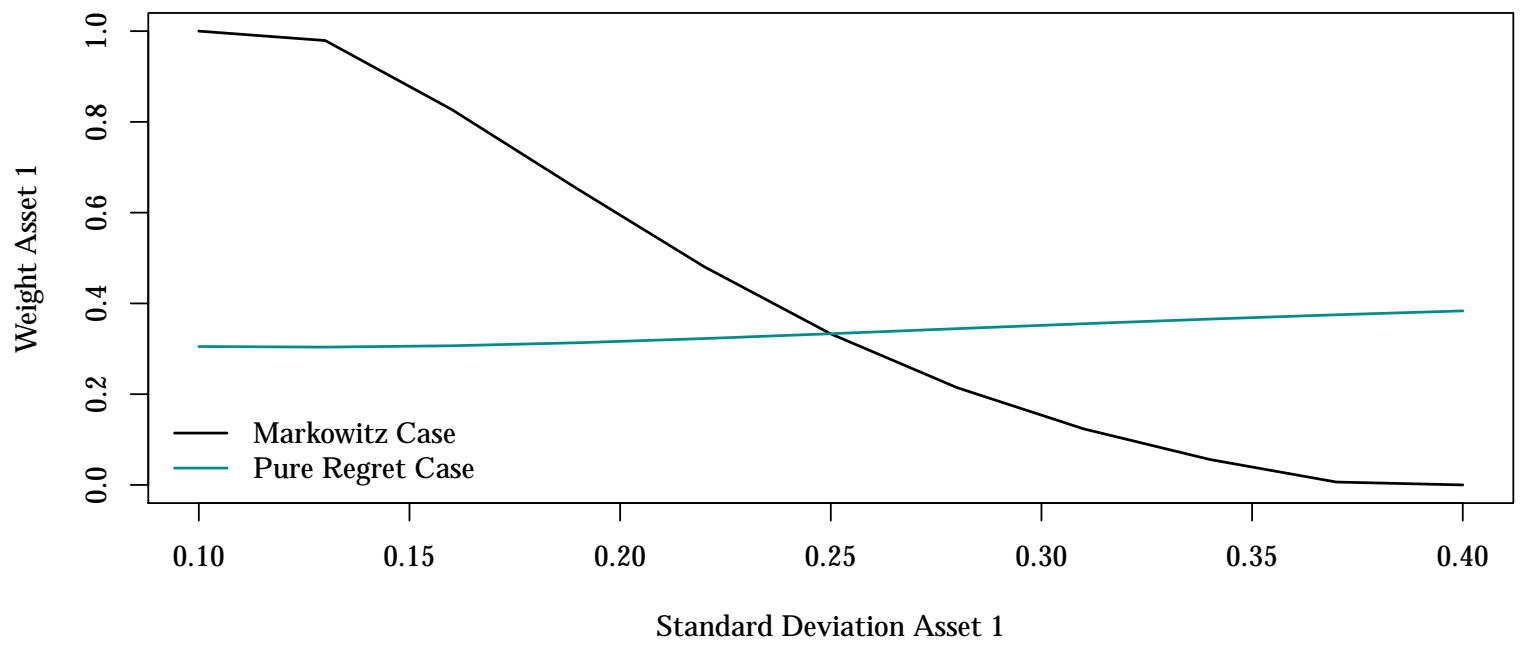

Part B: Portfolio Weight of Asset 1: Ten Assets

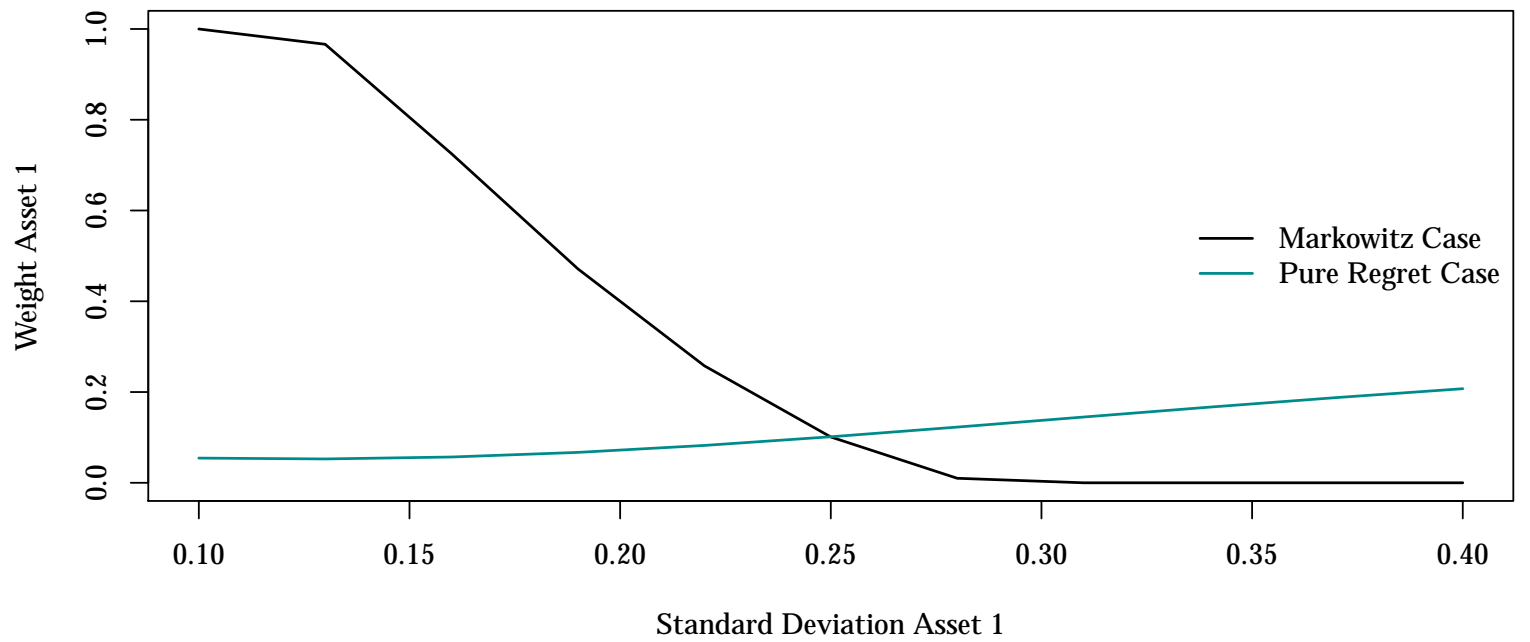

Note: Figure 3.5 shows the effects of a shift in the standard deviation of an asset (Asset 1) on its optimal portfolio weight in an investible universe with three assets (Part A) and ten assets (Part B). The base scenario uses three (ten) assets with a multivariate normal return distribution, identical expected returns of $10 \%$, identical standard deviations of $25 \%$, and identical correlations of 0.5 . The black lines refer to the Markowitz case $(\alpha=0)$ and the cyan lines to the pure regret case $(\alpha=1)$. 


\subsubsection{Correlation}

Another issue of the multi-asset case is to look at changes in correlations. In the two-asset case a comparative-static change in the correlation has no effect on portfolios weights if the assets have identical expected returns and standard deviations, leaving them fully homogeneous irrespective of their correlation. Only the multi-asset case allows us to introduce some heterogeneity in correlations that potentially has an impact on portfolio weights. To investigate correlation effects, we use the same base scenario as in Figure 3.5 and vary the correlation between Asset 1 and all other assets. Figure 3.6 provides the corresponding results. Again, Part A of the figure refers to the three-asset case and Part B to the ten-asset case. Both parts of the figure show the same tendency: The higher the correlation, the lower the portfolio weight. This result holds both for the Markowitz case and the pure regret case. The well-known intuition for the Markowitz case states that an asset that is highly correlated with other assets offers fewer diversification benefits. The same intuition works with respect to regret risk. Think of an asset with a low (even negative) correlation. This asset imposes a high regret risk on the other assets if it happens to be the ex-post best asset. To reduce the regret risk of the portfolio, one should therefore overweight this asset in comparison to a weight of $1 / \mathrm{N}$. The correlation effects in the pure regret case, however, are less pronounced than in the Markowitz case, suggesting that the introduction of regret into portfolio selection still mitigates correlation effects to some degree. 
Figure 3.6: Effects of a Shift in Correlation

Part A: Portfolio Weight of Asset 1: Three Assets

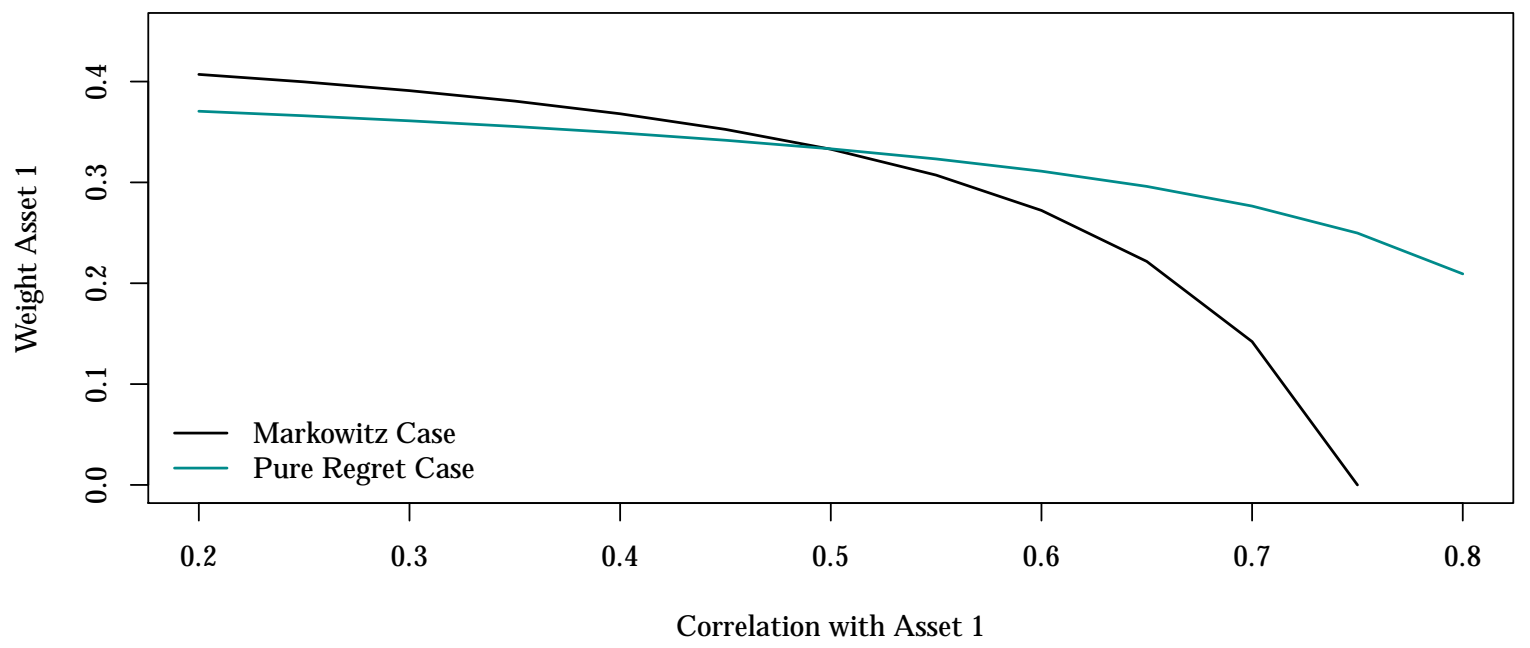

Part B: Portfolio Weight of Asset 1: Ten Assets

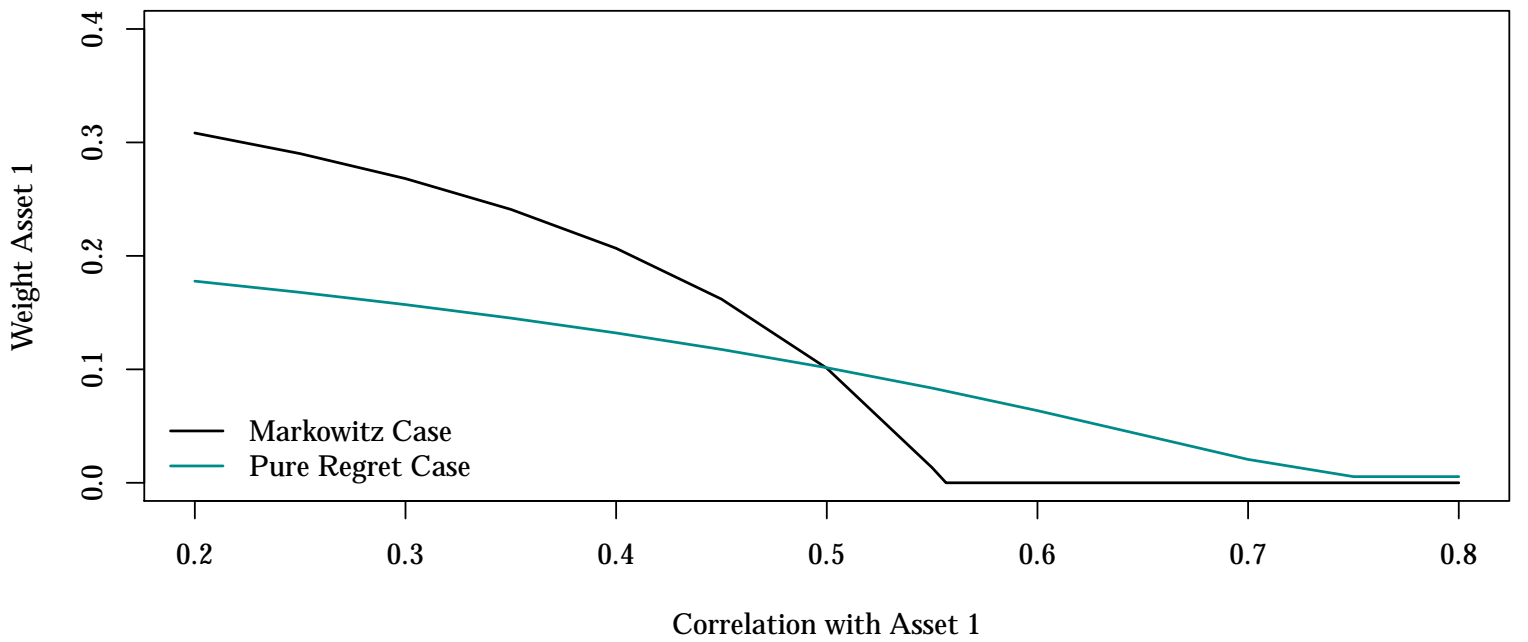

Note: Figure 3.6 shows the effects of a shift in the return correlation of an asset (Asset 1) with all other assets on its optimal portfolio weight in an investible universe with three assets (Part A) and ten assets (Part B). The base scenario uses three (ten) assets with a multivariate normal return distribution, identical expected returns of $10 \%$, identical standard deviations of $25 \%$, and identical correlations of 0.5 . The black lines refer to the Markowitz case $(\alpha=0)$ and the cyan lines to the pure regret case $(\alpha=1)$. 


\subsubsection{Summary}

As we have seen, the case of multiple assets adds complexity to the question of portfolio composition under the regret model. While the direction of an expected return effect is clear, this is not true for a shift in the standard deviation. Even if expected returns are equal among assets, suggesting a pure risk minimization, a higher standard deviation can lead to increasing or decreasing weights, depending on the correlation. Moreover, it has been shown that heterogeneous correlations also have an impact on regret risk and thereby on the resulting portfolio weights.

\subsection{Regret Effects for Real Portfolios}

\subsubsection{Data and Empirical Setup}

To study the impact of regret aversion on portfolio weights for real portfolios we use four different investible universes with a varying number of assets (funds). The universes represent different problems of asset allocation. These are concerned with the choice between stocks and bonds (two constituents), different levels of country development (three constituents), different investment styles (four constituents) and different continents (five constituents). More detailed information on the universes and the data that we use to obtain the necessary input parameters is given in Table 3.1. 
Table 3.1: Description of the Investible Universes

\begin{tabular}{|c|c|c|c|c|}
\hline Universe & Constituents & Period & Source & Specification \\
\hline $\begin{array}{l}\text { Stock- } \\
\text { Bond }\end{array}$ & $\begin{array}{l}\text { Stock Index } \\
\text { Bond Index }\end{array}$ & $\begin{array}{l}\text { Jan } 1988- \\
\text { Feb } 2017\end{array}$ & Datastream & $\begin{array}{l}\text { Russell } 1000 \text { as broad US } \\
\text { stock market index and } \\
\text { Bloomberg Barclays US } \\
\text { aggregated bond index }\end{array}$ \\
\hline Country & $\begin{array}{l}\text { Developed } \\
\text { BRIC } \\
\text { Emerging }\end{array}$ & $\begin{array}{l}\text { Jan } 1995- \\
\text { Feb } 2017\end{array}$ & Datastream & $\begin{array}{l}\text { Specification based on } \\
\text { Datastream's regional } \\
\text { indexes }\end{array}$ \\
\hline $\begin{array}{l}\text { Investment } \\
\text { Style }\end{array}$ & $\begin{array}{l}\text { Market } \\
\text { Size } \\
\text { Value } \\
\text { Momentum }\end{array}$ & $\begin{array}{l}\text { Nov } 1926- \\
\text { Dec } 2016\end{array}$ & $\begin{array}{l}\text { French } \\
\text { Library }\end{array}$ & $\begin{array}{l}\text { Calculation based on } \\
\text { CRSP universe }\end{array}$ \\
\hline Continent & $\begin{array}{l}\text { Latin America } \\
\text { North America } \\
\text { Asia } \\
\text { Europe } \\
\text { Pacific Basin }\end{array}$ & $\begin{array}{l}\text { Jul } 1994- \\
\text { Feb } 2017\end{array}$ & Datastream & $\begin{array}{l}\text { Specification based on } \\
\text { Datastream's regional } \\
\text { indexes }\end{array}$ \\
\hline
\end{tabular}

Note: Table 3.1 provides information about the investible universes and data sets used to obtain optimal portfolios. It shows the constituents of the universes, the data periods used to estimate input parameters, the data sources, and how the individual constituents (funds) are specified.

We determine optimal portfolios both for the Markowitz case $(\alpha=0)$ and the pure regret case $(\alpha=1)$. By concentrating on these two extreme cases we cover the full range of possible regret effects on portfolio composition. As for the simulated portfolios, we use a risk-aversion parameter of $\gamma=3$ for $\mu-\sigma$ portfolio optimization. ${ }^{12}$ In addition, the portfolio weights of minimum-variance portfolios based on the (unadjusted) return variance and the regret-adjusted variance, respectively, are determined. The reason is that the global minimum variance portfolio is the only efficient portfolio that can be formed without knowledge of expected returns. Because expected returns are difficult to estimate (Merton 1980), there is an ongoing interest in risk-minimizing strategies. All necessary input parameters are obtained from the corresponding sample moments using a daily data frequency. ${ }^{13}$ For each universe we report the mean return, the standard

\footnotetext{
${ }^{12}$ Other levels of risk aversion have been studied, but lead to the same conclusions.

${ }^{13}$ Estimation with weekly and monthly data has also been performed, but changes the results only marginally.
} 
deviation, the skewness, the regret adjustment of the expected return (covariance between the fund's return and the return of the ex-post best fund) according to Property 2, and the regret-adjusted standard deviation of each fund. Means and standard deviations are presented as annualized values. Moreover, we show the optimal portfolio weights resulting from the $\mu-\sigma$ optimization and the variance minimization both for the Markowitz case and the pure regret case. Because the relative attractiveness of an asset within a specific universe depends on how its return characteristics deviate from those of the other assets, we also present the deviations of individual funds' return characteristics from the average values of all funds.

\subsubsection{Results}

\subsubsection{Stock-Bond Universe}

Table 3.2 presents the asset characteristics and optimal portfolio weights for the stock-bond universe. Because the universe contains only two assets, it is a convenient starting point. Moreover, it directly relates to Harry Markowitz's statement in the introduction. If the investor's wealth is split up equally between stocks and bonds, does this really minimize regret? As Table 3.2 shows, the mean returns, standard deviations, and skewnesses of the two funds clearly differ. The mean return and the standard deviation are higher for the stock index, as one would expect. In the Markowitz case the stock index is more attractive and has a clearly higher weight than the bond index. In contrast, if variance minimization is considered, the larger part of the budget is invested in the bond index. The pure regret case shows a very different picture. The higher expected return and only slightly lower skewness make the stock index even more attractive. Moreover, the role of the (unadjusted) standard deviation is diminished. These effects show up in the relatively high regret adjustment for $\mu$. Actually, in terms of regret risk the two funds are almost equally risky, leading to an almost fifty-fifty allocation between indexes based on the minimization of the regret standard deviation. For the $\mu-\sigma$ optimization in the pure regret case the higher mean return of the stock index leads to a clear overweighting. In a nutshell, the main effects of asset characteristics on portfolio weights as described in Section 3.3 are clearly visible 
for this investible universe. Finally, the answer to the question relating to Markowitz's investment policy clearly depends on the relative importance of expected regret and regret risk: Splitting the wealth equally between stocks and bonds is not even close to the optimal solution for the pure regret case if expected regret plays a significant role, that is, if $\gamma$ is not very large. Stocks should then be strongly overweighted as compared to bonds. If the goal is to minimize regret risk, however, a fifty-fifty rule is very close to the optimal allocation. 
Table 3.2: Results for the Stock-Bond Universe

Panel A: Return Characteristics

\begin{tabular}{lcc}
\hline & Stock Index & Bond Index \\
\hline Mean Return & 0.1120 & 0.0606 \\
& $(0.0257)$ & $(-0.0257)$ \\
Std. Deviation & 0.1696 & 0.0702 \\
& $(0.0497)$ & $(-0.0497)$ \\
Skewness & -0.1411 & -0.0838 \\
& $(-0.0287)$ & $(0.0287)$ \\
Regret Adjustment $\mu$ & 0.0381 & 0.0049 \\
& $(0.0166)$ & $(-0.0166)$ \\
Regret-adj. Std. Dev. & 0.1252 & 0.1182 \\
& $(0.0035)$ & $(-0.0035)$ \\
\hline
\end{tabular}

Panel B: Portfolio Weights

\begin{tabular}{lccc}
\hline & & Stock Index & Bond Index \\
\hline \multirow{4}{*}{ Markowitz } & $\mu-\sigma(\gamma=3)$ & 0.6374 & 0.3626 \\
& & $(0.1374)$ & $(-0.1374)$ \\
& Min-Var & 0.1839 & 0.8161 \\
& & $(-0.3161)$ & $(0.3161)$ \\
Pure Regret & & & \\
& & & \\
& & 0.9312 & 0.0688 \\
& & $(0.4312)$ & $(-0.4312)$ \\
& & & 0.5224 \\
& & 0.4776 & $(0.0224)$ \\
\hline
\end{tabular}

Note: Table 3.2 shows different return characteristics for the stock-bond universe (Panel A) and the corresponding optimal portfolio weights in the Markowitz case $(\alpha=0)$ and the pure regret case $(\alpha=1)$ (Panel B). The investible universe consists of two assets: one fund with stocks (stock index) and one fund with bonds (bond index). All presented values are absolute figures with the deviations from their mean in parentheses below each value. Mean return, standard deviation, and skewness are calculated for the unadjusted returns of each asset. The regret-adjustment for $\mu$ is the covariance between the asset's return and the return of the ex-post best asset. It can be transferred into any investor-specific adjustment by multiplying it with $2 \alpha \gamma$. The regret-adjusted standard deviation is the standard deviation of regret-adjusted returns in the pure regret case. All results are based on daily data and (except for the skewness) all return characteristics are provided as annualized values. 


\subsubsection{Country Universe}

The country universe provides an example with three assets and the corresponding results are presented in Table 3.3. The BRIC fund has the highest expected return and the highest return standard deviation, whereas the EME fund has the lowest expected returns and the DEV fund has the lowest standard deviation. The low standard deviation makes the DEV fund very attractive in the Markowitz case, leading to a portfolio weight of more than two thirds. Depending on the importance of a high mean ( $\mu-\sigma$ optimization) or a low standard deviation (variance minimization), the remaining budget is either invested in the BRIC fund or the EME fund, respectively. When moving to the pure regret case, we see a clear reduction in the position held in the DEV fund, reflecting the diminishing importance of the standard deviation. We also observe higher weights for the BRIC fund, the fund with the highest mean returns. Again, we see that regret-averse investors care less about risk in terms of return volatility and are therefore willing to invest more in the quite volatile BRIC fund. 
Table 3.3: Results for the Country Universe

Panel A: Return Characteristics

\begin{tabular}{lccc}
\hline & DEV & EME & BRIC \\
\hline Mean Return & 0.0827 & 0.0762 & 0.1053 \\
& $(-0.0054)$ & $(-0.0119)$ & $(0.0172)$ \\
Std. Deviation & 0.1473 & 0.1683 & 0.2202 \\
& $(-0.0313)$ & $(-0.0103)$ & $(0.0416)$ \\
Skewness & & & \\
& -0.2767 & -0.5831 & -0.3639 \\
Regret Adjustment $\mu$ & 0.0588 & 0.0723 & 0.0975 \\
& $(-0.0174)$ & $(-0.0039)$ & $(0.0213)$ \\
Regret-adj. Std. Dev. & 0.1043 & 0.0916 & 0.1082 \\
& $(0.0029)$ & $(-0.0098)$ & $(0.0068)$ \\
\hline
\end{tabular}

Panel B: Portfolio Weights

\begin{tabular}{|c|c|c|c|c|}
\hline & & DEV & EME & BRIC \\
\hline \multirow{2}{*}{ Markowitz } & $\mu-\sigma(\gamma=3)$ & $\begin{array}{c}0.6999 \\
(0.3666)\end{array}$ & $\begin{array}{c}0.0000 \\
(-0.3333)\end{array}$ & $\begin{array}{c}0.3001 \\
(-0.0332)\end{array}$ \\
\hline & Min-Var & $\begin{array}{c}0.7110 \\
(0.3777)\end{array}$ & $\begin{array}{c}0.2890 \\
(-0.0443)\end{array}$ & $\begin{array}{c}0.0000 \\
(-0.3333)\end{array}$ \\
\hline \multirow{2}{*}{ Pure Regret } & $\mu-\sigma(\gamma=3)$ & $\begin{array}{c}0.2578 \\
(-0.0755)\end{array}$ & $\begin{array}{c}0.0000 \\
(-0.3333)\end{array}$ & $\begin{array}{c}0.7422 \\
(0.4089)\end{array}$ \\
\hline & Min-Var & $\begin{array}{c}0.4278 \\
(0.0945)\end{array}$ & $\begin{array}{c}0.1903 \\
(-0.1430)\end{array}$ & $\begin{array}{c}0.3819 \\
(0.0486)\end{array}$ \\
\hline
\end{tabular}

Note: Table 3.3 shows different return characteristics for the country universe (Panel A) and the corresponding optimal portfolio weights in the Markowitz case $(\alpha=0)$ and the pure regret case $(\alpha=1)$ (Panel B). The investible universe consists of three assets: one fund with stocks from developed markets (DEV), one fund with stocks from emerging markets (EME), and one fund with stocks from the BRIC countries (BRIC). All presented values are absolute figures with the deviations from their mean in parentheses below each value. Mean return, standard deviation, and skewness are calculated for the unadjusted returns of each asset. The regret-adjustment for $\mu$ is the covariance between the asset's return and the return of the ex-post best asset. It can be transferred into any investor-specific adjustment by multiplying it with $2 \alpha \gamma$. The regret-adjusted standard deviation is the standard deviation of regret-adjusted returns in the pure regret case. All results are based on daily data and (except for the skewness) all return characteristics are provided as annualized values. 


\subsubsection{Investment Style Universe}

The results for the investment style universe are presented in Table 3.4. Here we observe that the portfolio weights of two of the funds (SMB and HML) hardly change when moving from the Markowitz case to the pure regret case. Substantial changes, however, occur to the Market fund ( $\mu-\sigma$ optimization and variance minimization) and the MOM fund ( $\mu-\sigma$ optimization). The Market fund is extreme in the sense that it has the highest mean return and the highest standard deviation. As the impact of the expected return on optimal portfolio weights is stronger and the impact of the standard deviation weaker in the pure regret case, we should see the weights of the Market fund rise, which is exactly what we do observe. An explanation for the shrinking weight of the MOM fund with $\mu-\sigma$ optimization lies in its relative advantages and disadvantages as compared to the Market fund. It has a disadvantage in terms of mean returns but an advantage in terms of standard deviation. However, this advantage counts less (if at all) in the pure regret case, leading to the lower weight. 
Table 3.4: Results for the Investment Style Universe

Panel A: Return Characteristics

\begin{tabular}{lcccc}
\hline & Market & SMB & HML & MOM \\
\hline Mean Return & 0.0726 & 0.014 & 0.0431 & 0.0671 \\
& $(0.0234)$ & $(-0.0352)$ & $(-0.0061)$ & $(0.0179)$ \\
Std. Deviation & 0.1697 & 0.0931 & 0.0930 & 0.1191 \\
& $(0.0511)$ & $(-0.0255)$ & $(-0.0256)$ & $(0.0005)$ \\
Skewness & & & & \\
& -0.1124 & -0.7698 & 0.7451 & -1.6148 \\
& $(0.3256)$ & $(-0.3318)$ & $(1.1831)$ & $(-1.1768)$ \\
Regret Adjustment $\mu$ & 0.0190 & -0.0054 & 0.0012 & -0.0033 \\
& $(0.0161)$ & $(-0.0083)$ & $(-0.0017)$ & $(-0.0062)$ \\
Regret-adj. Std. Dev. & 0.1575 & 0.1450 & 0.1286 & 0.1582 \\
& $(0.0102)$ & $(-0.0023)$ & $(-0.0187)$ & $(0.0109)$ \\
\hline
\end{tabular}

Panel B: Portfolio Weights

\begin{tabular}{|c|c|c|c|c|c|}
\hline & & Market & SMB & HML & MOM \\
\hline \multirow{2}{*}{ Markowitz } & $\mu-\sigma(\gamma=3)$ & $\begin{array}{c}0.3857 \\
(0.1357)\end{array}$ & $\begin{array}{c}0.0000 \\
(-0.2500)\end{array}$ & $\begin{array}{c}0.0000 \\
(-0.2500)\end{array}$ & $\begin{array}{c}0.6143 \\
(0.3643)\end{array}$ \\
\hline & Min-Var & $\begin{array}{c}0.1107 \\
(-0.1393)\end{array}$ & $\begin{array}{c}0.3366 \\
(0.0866)\end{array}$ & $\begin{array}{c}0.3259 \\
(0.0759)\end{array}$ & $\begin{array}{c}0.2268 \\
(-0.0232)\end{array}$ \\
\hline \multirow{2}{*}{ Pure Regret } & $\mu-\sigma(\gamma=3)$ & $\begin{array}{c}0.5402 \\
(0.2902)\end{array}$ & $\begin{array}{c}0.0000 \\
(-0.2500)\end{array}$ & $\begin{array}{c}0.0000 \\
(-0.2500)\end{array}$ & $\begin{array}{c}0.4598 \\
(0.2098)\end{array}$ \\
\hline & Min-Var & $\begin{array}{c}0.3177 \\
(0.0677)\end{array}$ & $\begin{array}{c}0.1975 \\
(-0.0525)\end{array}$ & $\begin{array}{c}0.2943 \\
(0.0443)\end{array}$ & $\begin{array}{c}0.1905 \\
(-0.0595)\end{array}$ \\
\hline
\end{tabular}

Note: Table 3.4 shows different return characteristics for the investment style universe (Panel A) and the corresponding optimal portfolio weights in the Markowitz case $(\alpha=0)$ and the pure regret case $(\alpha=1)$ (Panel B). The investible universe consists of four assets: one fund representing the market portfolio (Market), one fund representing the size factor (SMB), one fund representing the value factor (HML), and one fund representing the momentum factor (MOM). All presented values are absolute figures with the deviations from their mean in parentheses below each value. Mean return, standard deviation, and skewness are calculated for the unadjusted returns of each asset. The regret-adjustment for $\mu$ is the covariance between the asset's return and the return of the ex-post best asset. It can be transferred into any investor-specific adjustment by multiplying it with $2 \alpha \gamma$. The regret-adjusted standard deviation is the standard deviation of regret-adjusted returns in the pure regret case. All results are based on daily data and (except for the skewness) all return characteristics are provided as annualized values. 


\subsubsection{Continents Universe}

Table 3.5 shows the results for our last example, the continents universe consisting of five assets. From this universe we can learn different things. First, we see again that the effects of a move from the Markowitz case to the pure regret case on optimal portfolio weights can be very large. If we consider the LA fund, the Markowitz investor would not invest at all, whereas the regret-averse investor would even overweight this fund. The reason clearly is the very high standard deviation of the fund. Second, it can well be that two assets with apparently similar return characteristics react quite differently. An example are the AS and PB funds, which have very similar (univariate) return characteristics. The weight of the AS fund is reduced substantially in the pure regret case as compared to the Markowitz case (variance minimization). In contrast, the weight of the PB fund increases substantially. This result emphasizes the importance of the co-movement of the assets' returns, which can well be different for the (unadjusted) returns and the regret-adjusted returns. Third, an above average regret adjustment for $\mu$ is not generally an indication of an increasing portfolio weight when moving to the pure regret case (see the EU fund). The reason is that the specific structure of the adjustments of all other assets also plays a role. Finally, the regret effects can be substantially different for $\mu-\sigma$ optimization than for variance minimization. Portfolio weights might well increase in the former case and decrease in the latter, as can be seen for the PB fund. 
Table 3.5: Results for the Continent Universe

Panel A: Return Characteristics

\begin{tabular}{lccccc}
\hline & LA & NA & AS & EU & PB \\
\hline Mean Return & 0.0976 & 0.1078 & 0.0439 & 0.0922 & 0.0468 \\
& $(0.0199)$ & $(0.0301)$ & $(-0.0338)$ & $(0.0145)$ & $(-0.0309)$ \\
Std. Deviation & 0.2296 & 0.1812 & 0.1788 & 0.1894 & 0.1794 \\
& $(0.0379)$ & $(-0.0105)$ & $(-0.0129)$ & $(-0.0023)$ & $(-0.0123)$ \\
Skewness & & & & & \\
& -0.3689 & -0.1733 & -0.0759 & -0.1815 & -0.1043 \\
Regret Adjustment $\mu$ & $(-0.1881)$ & $(0.0075)$ & $(0.1049)$ & $(-0.0007)$ & $(0.0765)$ \\
& 0.0831 & 0.0573 & 0.0486 & 0.0642 & 0.0495 \\
& $(0.0226)$ & $(-0.0032)$ & $(-0.0119)$ & $(0.0037)$ & $(-0.0110)$ \\
Regret-adj. Std. Dev. & 0.1649 & 0.1571 & 0.1721 & 0.1523 & 0.1711 \\
& $(0.0014)$ & $(-0.0064)$ & $(0.0086)$ & $(-0.0112)$ & $(0.0076)$ \\
\hline
\end{tabular}

Panel B: Portfolio Weights

\begin{tabular}{|c|c|c|c|c|c|c|}
\hline & & LA & NA & AS & EU & PB \\
\hline \multirow{2}{*}{ Markowitz } & $\mu-\sigma(\gamma=3)$ & $\begin{array}{c}0.0000 \\
(-0.2000)\end{array}$ & $\begin{array}{c}0.7078 \\
(0.5078)\end{array}$ & $\begin{array}{c}0.0000 \\
(-0.2000)\end{array}$ & $\begin{array}{c}0.2704 \\
(0.0704)\end{array}$ & $\begin{array}{c}0.0218 \\
(-0.1782)\end{array}$ \\
\hline & Min-Var & $\begin{array}{c}0.0000 \\
(-0.2000)\end{array}$ & $\begin{array}{c}0.4186 \\
(0.2186)\end{array}$ & $\begin{array}{c}0.4565 \\
(0.2565)\end{array}$ & $\begin{array}{c}0.1249 \\
(-0.0751)\end{array}$ & $\begin{array}{c}0.0000 \\
(-0.2000)\end{array}$ \\
\hline \multirow{2}{*}{ Pure Regret } & $\mu \sigma(\gamma=3)$ & $\begin{array}{c}0.2696 \\
(0.0696)\end{array}$ & $\begin{array}{c}0.5108 \\
(0.3108)\end{array}$ & $\begin{array}{c}0.0000 \\
(-0.2000)\end{array}$ & $\begin{array}{c}0.2195 \\
(0.0195)\end{array}$ & $\begin{array}{c}0.0000 \\
(-0.2000)\end{array}$ \\
\hline & Min-Var & $\begin{array}{c}0.2533 \\
(0.0533)\end{array}$ & $\begin{array}{c}0.2662 \\
(0.0662)\end{array}$ & $\begin{array}{c}0.1588 \\
(-0.0412)\end{array}$ & $\begin{array}{c}0.1627 \\
(-0.0373)\end{array}$ & $\begin{array}{c}0.1589 \\
(-0.0411)\end{array}$ \\
\hline
\end{tabular}

Note: Table 3.5 shows different return characteristics for the continent universe (Panel A) and the corresponding optimal portfolio weights in the Markowitz case $(\alpha=0)$ and the pure regret case $(\alpha=1)$ (Panel B). The investible universe consists of five assets: one fund with stocks from Latin America (LA), one fund with stocks North America (NA), one fund with stocks from (AS), one fund with stocks from Europe (EU), and one fund with stocks from the Pacific Basin (PB). All presented values are absolute figures with the deviations from their mean in parentheses below each value. Mean return, standard deviation, and skewness are calculated for the unadjusted returns of each asset. The regret-adjustment for $\mu$ is the covariance between the asset's return and the return of the ex-post best asset. It can be transferred into any investor-specific adjustment by multiplying it with $2 \alpha \gamma$. The regret-adjusted standard deviation is the standard deviation of regret-adjusted returns in the pure regret case. All results are based on daily data and (except for the skewness) all return characteristics are provided as annualized values. 


\subsubsection{Summary}

In summary, the examples of problems in real asset allocation have demonstrated that the quantitative effects of regret risk on optimal portfolios can be very large. Moreover, the intuition from Section 3.3 about which return characteristics make an asset more or less attractive in the regret world as compared to the Markowitz world are broadly confirmed. However, our analysis also shows that it is not always easy to anticipate the effects of regret based on some simple univariate indicators, which highlights the importance of a formal yet tractable optimization procedure.

\subsection{Conclusion}

This paper has extended Markowitz's portfolio selection by considering investors' regret as an additional decision criterion beyond final wealth. Even with this additional criterion the approach keeps the simplicity and tractability of the Markowitz model. As has been shown, regret affects optimal portfolios as compared to the Markowitz model only because it leads to a different risk measure. However, this risk measure does not only depend on the portfolio's return variance but also on the expected return and skewness. These results demonstrate that the regret-averse investor, seeking to be close to the ex-post best asset, has a distinctively different view on risk than the traditional mean-variance investor. This different view can have a strong impact on optimal portfolio weights, which has been shown for several examples of real portfolios. Moreover, the notion of a risk-free asset is a different one in the regret model, because no asset exists that is free of regret risk. Therefore, we do not have to distinguish between risk-free and risky assets in our approach.

It was our intention to stay as close as possible to the Markowitz model in our analysis. However, different extension could be considered. A first one would go beyond the mean and variance of regret-adjusted returns in the preference function (Equation 3.2) and consider higher-order moments like skewness and kurtosis. Formally, such an extension is straightforward. However, it would increase the number of input parameters considerably and make the interpretation of the results more difficult. Another natural variation of the 
preference function would be to replace the variance of regret-adjusted returns by some measure of downside risk, like lower-partial moments. Finally, one could consider extending the investible universe to non-linear instruments. As suggested by the results of Muermann et al. (2006) on return guarantees in defined contribution pension plans and Korn and Rieger (2017) on optimal hedging instruments for corporate hedging under regret, such non-linear instruments could be valuable for investors who are concerned about regret. 


\section{A Appendix}

\section{A.1 Optimal Portfolio Weights}

The classical optimal portfolio is obtained by maximizing the preference function

$$
\pi\left(R_{P}\right)=\omega^{t r} \mu-\gamma \omega^{t r} \Sigma \omega
$$

subject to the budget constraint

$$
\omega^{\operatorname{tr}} \underline{1}=1
$$

Differentiating the Lagrange function

$$
L(\omega)=\omega^{t r} \mu-\gamma \omega^{t r} \Sigma \omega-\lambda\left(\omega^{t r} \underline{1}-1\right)
$$

with respect to $\omega$ yields

$$
\frac{\partial L(\omega)}{\partial \omega}=\mu-2 \gamma \Sigma \omega-\lambda \underline{1}
$$

thus

$$
\omega^{*}=\frac{1}{2 \gamma} \Sigma^{-1}(\mu-\lambda \underline{1})
$$

The Lagrange parameter $\lambda$ is obtained by differentiating $L$ with respect to $\lambda$ :

$$
\begin{aligned}
& \frac{\partial L(\omega)}{\partial \lambda}=\omega^{t r} \underline{1}-1 \\
& =\frac{1}{2 \gamma} \mu^{t r} \Sigma^{-1} \underline{1}-\frac{\lambda}{2 \gamma} \underline{1}^{t r} \Sigma^{-1} \underline{1}-1,
\end{aligned}
$$

yielding

$$
\lambda=\frac{\underline{1}^{t r} \Sigma^{-1} \mu-2 \gamma}{\underline{1}^{t r} \Sigma^{-1} \underline{1}} .
$$

Thus

$$
\omega^{*}=\frac{1}{2 \gamma} \Sigma^{-1}\left(\mu-\frac{\underline{1}^{t r} \Sigma^{-1} \mu-2 \gamma}{\underline{1}^{t r} \Sigma^{-1} \underline{1}} \underline{1}\right) .
$$


For obtaining the optimal portfolio under the regret model, we can use the representation of the preference function from Equation 3.7

$$
\pi\left(Z_{P}\right)=\omega^{t r} \mu_{r a}-\gamma \omega^{t r} \Sigma \omega-c
$$

with

$$
\mu_{\text {ra }}=\mu+2 \alpha \gamma \operatorname{Cov}\left(R, R_{\max }\right) .
$$

Analogously to the classical case we get

$$
\omega_{r a}^{*}=\frac{1}{2 \gamma} \Sigma^{-1}\left(\mu_{r a}-\frac{\underline{1}^{t r} \Sigma^{-1} \mu_{r a}-2 \gamma}{\underline{1}^{t r} \Sigma^{-1} \underline{1}} \underline{1}\right) .
$$

\section{A.2 Impact of Expected Return Changes on Optimal Portfolio Weights}

We look at the sensitivity of the optimal portfolio weights with respect to a change in the mean vector $\mu$. In the classical case we can calculate the first derivative as

$$
\begin{aligned}
& \frac{\partial \omega^{*}}{\partial \mu}=\left(\frac{\partial \omega_{i}^{*}}{\partial \mu_{j}}\right)_{i, j} \\
& =\frac{1}{2 \gamma} \Sigma^{-1}-\frac{1}{2 \gamma} \Sigma^{-1} \frac{1}{\underline{1}^{\operatorname{tr}} \Sigma^{-1}} \\
& =\frac{1}{2 \gamma} \Sigma^{-1}\left(I-\frac{\underline{1}^{t r} \Sigma^{-1}}{\underline{1}^{t r} \Sigma^{-1} \underline{1}}\right) \text {, }
\end{aligned}
$$

where $I$ is the identity matrix. This value of the derivative is a constant, independent of $\mu$. Thus, the optimal weight of an asset increases linearly with its mean in the classical model. In the regret model we have

$$
\frac{\partial \omega_{r a, i}^{*}}{\partial \mu_{j}}=\frac{\partial \omega_{r a, i}^{*}}{\partial \mu_{r a, j}} \cdot \frac{\partial \mu_{r a, j}}{\partial \mu_{j}},
$$

with

$$
\frac{\partial \omega_{r a, i}^{*}}{\partial \mu_{r a, j}}=\frac{\partial \omega_{i}^{*}}{\partial \mu_{j}}
$$


as above. Furthermore,

$$
\frac{\partial \mu_{r a, j}}{\partial \mu_{j}}=\frac{\partial}{\mu_{j}}\left(\mu_{j}+2 \alpha \gamma \operatorname{Cov}\left(R_{j}, R_{\max }\right)\right)=1+2 \alpha \gamma \frac{\partial}{\partial \mu_{j}} \operatorname{Cov}\left(R_{j}, R_{\text {max }}\right) .
$$

To analyze the derivative of the covariance term, let us assume that the return vector $R$ has a joint elliptical distribution with density $f(\cdot ; \mu, \Sigma)$. Then,

$$
\begin{aligned}
\operatorname{Cov}\left(R_{j}, R_{\text {max }}\right) & =E\left[R_{j} \cdot R_{\text {max }}\right]-\mu_{j} \cdot E\left[R_{\text {max }}\right] \\
& =\int \cdots \int\left(R_{j} \cdot \max _{k}\left\{R_{k}\right\}-\mu_{j} \cdot \max _{k}\left\{R_{k}\right\}\right) \cdot f(R ; \mu, \Sigma) d R_{n} \cdots d R_{1} \\
& =\int \cdots \int\left(\left(R_{j}+\mu_{j}\right) \cdot \max _{k}\left\{R_{k}+\mu_{k}\right\}-\mu_{j} \cdot \max _{k}\left\{R_{k}+\mu_{k}\right\}\right) . \\
& \quad f(R ; 0, \Sigma) d R_{n} \cdots d R_{1} \\
& =\int \cdots \int R_{j} \cdot \max _{k}\left\{R_{k}+\mu_{k}\right\} \cdot f(R ; 0, \Sigma) d R_{n} \cdots d R_{1}
\end{aligned}
$$

with substitution $\tilde{R}=R-\mu$. Differentiating under the integral, we obtain

$$
\begin{aligned}
\frac{\partial \operatorname{Cov}\left(R_{j}, R_{\text {max }}\right)}{\partial \mu_{j}} & =\int \cdots \int \frac{\partial}{\partial \mu_{j}} R_{j} \cdot \max _{k}\left\{R_{k}+\mu_{k}\right\} \cdot f(R ; 0, \Sigma) d R_{n} \cdots d R_{1} \\
& =\int \cdots \int R_{j} \cdot 1_{R_{j}=\max _{k}\left\{R_{k}+\mu_{k}\right\}} \cdot f(R ; 0, \Sigma) d R_{n} \cdots d R_{1} \\
& =\int \cdots \int\left(R_{j}-\mu_{j}\right) \cdot 1_{R_{j}=\max _{k}\left\{R_{k}\right\}} \cdot f(R ; \mu, \Sigma) d R_{n} \cdots d R_{1} \\
& =E\left[\left(R_{j}-\mu_{j}\right) \cdot 1_{R_{j}=\max _{k}\left\{R_{k}\right\}}\right] \\
& =E\left[R_{j}-\mu_{j} \mid R_{j}=\max _{k}\left\{R_{k}\right\}\right] \cdot P\left(R_{j}=\max _{k}\left\{R_{k}\right\}\right)
\end{aligned}
$$

with re-substitution $R=\tilde{R}+\mu$.

As $E\left[\left(R_{j}-\mu_{j}\right)\right]=0$, the expectation conditional on $R_{j}=\max _{k}\left\{R_{k}\right\}$ is greater than zero. Hence, the sensitivity of the portfolio weight with respect to the expectation $\mu$ in the regret model,

$$
\frac{\partial \omega_{r a, i}^{*}}{\partial \mu_{j}}=\frac{\partial \omega_{i}^{*}}{\partial \mu_{j}} \cdot\left(1+2 \alpha \gamma E\left[R_{j}-\mu_{j} \mid R_{j}=\max _{k}\left\{R_{k}\right\}\right] P\left(R_{j}=\max _{k}\left\{R_{k}\right\}\right)\right)
$$

is always larger than the sensitivity in the classical model. 


\title{
4 Beta Dispersion and Market Timing
}

under Review at the Journal of Financial Markets

\begin{abstract}
The beta dispersion, which is the spread of betas on a stock market, can be interpreted as a measure of market vulnerability. This study examines the economic idea of the beta dispersion and its application as a market return predictor. Based on the empirical beta dispersion observed in the US equity market, the study develops measures to predict future market returns. These dispersion measures have substantial predictive power for future market movements. Moreover, I show that the informational content of beta dispersion can be successfully exploited by market timing strategies with the help of distributional regressions. This is an innovative application of this novel way of modeling the relationship between multiple variables. The market timing strategies considerably enhance the riskreturn characteristics, especially by reducing the return volatility.
\end{abstract}

\footnotetext{
Acknowledgment: Earlier versions of this article have been presented at the 2016 Applied Financial Modeling Conference, Melbourne, the 2016 SGF Conference, Zürich, the 2016 EFMA Meeting, Basel, the 2016 FMA Meetings, Las Vegas, the research colloquium of Georg-August-Universität Göttingen 2016, the 2017 AFFI conference, Valence, the Ph.D. workshop of the 2017 Meeting of the German Finance Association, Ulm, and the 2017 Australasian Finance \& Banking Conference, Sydney. I am grateful to the discussants and participants of each seminar for their helpful comments and suggestions.
} 


\subsection{Introduction}

The prediction of the market risk premium has been receiving considerable attention from both academics and investors. Over the past decades, a growing number of studies have developed macroeconomic and technical indicators that can forecast the market risk premium, at least to a certain extent (e.g., Ang and Bekaert (2007); Campbell and Thompson (2008); Rapach and Zhou (2013); Neely et al. (2014)). The precise prediction of future returns is almost impossible, but even reliable predictions of the sign of the future return can be very valuable to different stakeholders. It helps investors to allocate their assets, it allows for the cost of capital calculation in business valuation, and it can support supervisors in monitoring the financial stability of markets. This study introduces beta dispersion as a novel and successful predictor of the market return and provides a coherent economic explanation on this measure. The study also presents an innovative empirical implementation of a market timing strategy to show that the predictions are useful to investors. Besides this investment perspective, the introduced beta dispersion captures a facet of the systemic risk, which may contribute toward the quantification of financial stability by supervisors.

The beta dispersion, which is defined as the time-varying spread of stock betas, can be interpreted as a measure of risk for market declines, particularly, the magnitude of such a decline. The more the betas are dispersed, that is, the more the number of stocks with extremely high and low betas that are traded in a market, the more inhomogeneously the stocks will be affected by and react to a systematic shock. These market-wide shocks are inherently precarious for companies with high betas because such companies are hit seriously. Consequently, these shocks cause a sharp decline in the fundamentals of companies (e.g., sales and profit, among others), thereby increasing insolvency risks. Moreover, the collapse of these companies might trigger contagion effects for comparable and interconnected companies (e.g., suppliers), and this scenario might lead to an increase in the level of financial distress risk in the whole market. This can be inferred to as an endogenous shock caused by an initial exogenous shock and can be interpreted as the subsequent systematic shock. In comparison, a market with a narrow beta dispersion would be more likely to 
experience only the initial systematic shock. Therefore, beta dispersion should provide a measure for a market's vulnerability to systematic shocks. It must indicate the probability and severity of the expected market decline, and, consequently, should be able to predict future market returns. In this sense, beta dispersion can be linked to financial stability. If beta dispersion is a valuable predictor of the probability of market declines and, especially, their magnitude, then the level of beta dispersion can indicate the ability of a market to cope with systematic shocks. Therefore, beta dispersion can also serve as a measure for systemic risks and the stability of the financial market.

The economic concept of beta dispersion is theoretically embedded in the conditional capital asset pricing model (CAPM). Instead of the assumption in the original CAPM that stocks have a constant sensitivity towards systematic risk, the conditional version allows for a time-varying beta coefficient. A stochastic beta has substantial impacts on the expected returns of assets, as the covariance between beta and the market return has to be considered (Jagannathan and Wang 1996). In this framework, it can be inferred that the time-varying beta may contain information about the future market risk premium. The economic idea of the beta dispersion helps to determine portfolios for which this link between beta and future market return should be existent. Quantifying this link is the initial evidence for the predictive relationship between beta dispersion and market return. The empirical documentation and evaluation of this connection is the starting point for the following analyses of beta dispersion as a measure of market vulnerability.

This study contributes by being the first study to show empirically that the beta coefficient co-varies with the future market return and by being the first study to provide an indepth analysis as well as an economic justification for this phenomenon. Based on this, a reasonable measure for beta dispersion as a measure of the vulnerability of a market, in terms of the probability and the extent of future market declines, is derived. This measure is studied and quantified empirically in a comprehensive way. Predictive regressions confirm the ability of beta dispersion to predict the future market return. The results can be verified by controlling for other well-known market return predictors, such as the cay factor, dividend yield, and short rate. Importantly, beta dispersion is also a valuable predictor of market return out-of-sample. It can clearly compete with and extend the 
already known predictors of the market risk premium. Hence, it can be stated that beta dispersion improves the forecasting accuracy of the future market return.

The study also contributes toward the improvement of timing strategies by introducing distributional regressions to the implementation of portfolio strategies. Beta dispersion is used as conditional information to estimate the distribution of the future market return. The probability that the market return will be positive is used as a decision criterion whether to invest in the market. This approach leads to a strong reduction in the return volatility of the resulting timing strategies and beats the benchmarks, in terms of return per unit of risk. The study shows that investors can profit from the predictive relationship between beta dispersion and market return. It is seen that the concept of distributional regressions is promising in the financial context and can be modified to other models of prediction for enhancing timing strategies.

First, this study is related to the literature on the prediction of market returns and the equity premium (among others Lettau and Ludvigson (2001); Goyal and Welch (2003); Lewellen (2004); Avramov and Chordia (2006); Ang and Bekaert (2007); Spiegel (2008); Cochrane (2008); Campbell and Thompson (2008); Pollet and Wilson (2010); Rapach et al. (2010)). Welch and Goyal (2008) provide a comprehensive overview of the most common macroeconomic predictors of the market return. They conclude that most predictors perform poorly out-of-sample and would not help an investor to profit from forecasts. Their analysis concludes that the historical mean of the market return seems to be the most stringent and successful predictor. Neely et al. (2014) as well as Baetje and Menkhoff (2016) compare macroeconomic predictors to technical indicators typically used by practitioners and reason that the latter perform just as well as macroeconomic predictors in- and out-of-sample. My study adds to the literature by demonstrating that beta dispersion contains unregarded information about future market returns and can successfully predict the market return in- and out-of-sample. Additionally, the study adds beta dispersion to the existing range of predictors of market return increasing the predictive accuracy of the market return.

As the definition of beta dispersion is inspired by the concept of return dispersion, this study is related to the literature on return dispersion. The return dispersion is a measure 
of the cross-sectional variability in stock returns at a certain point in time. A high return dispersion occurs in an economic recession and is positively related to the volatility level of the market and the momentum factor (Bekaert and Harvey 2000; Christie and Huang 1995; Connolly and Stivers 2003; Stivers 2003; Jiang 2010). Maio (2016) explicitly studies return dispersion as the predictor of the future market return and confirms this connection. Contrarily, this study focuses on the cross-sectional standard deviation of betas, which resembles return dispersion, but explicitly focuses on systematic risk. Additionally, the interpretation of beta dispersion as a market return predictor differs from that of return dispersion. The beta dispersion is constructed to be a measure of systemic imbalances in the market, and, therefore, predicts stumbles of the market, while return dispersion is an alternative measure of volatility. My study shows that beta dispersion and return dispersion complement each other and can co-exist in predictive analyses.

Finally, this study is related to market timing strategies and their performance evaluation, which have been extensively discussed in the literature (Jeffrey 1984; Pfeifer 1985; Bauer Jr. and Dahlquist 2001; Kostakis et al. 2011; Neuhierl and Schlusche 2011; Hallerbach 2014; Dichtl et al. 2016). To determine the allocation between stock and money market, technical, macroeconomic, and sentiment indicators have been introduced and tested for their ability to time the market (Brock et al. 1992; Shen 2003; Chen 2009; Feldman et al. 2015). Unlike stock selection strategies, market timing strategies try to achieve an outperformance only by driving investment in the market portfolio during market upturns and prevent investments during market downturns without any selection of single assets (Sharpe 1975). This study introduces a new possibility of differentiating between upturns and downturns with the help of distributional regression and chooses a careful investment approach that also considers the probability of a positive market return. The introduced strategies outperform the benchmark, even when the performance is accounted for general shortcomings of market timing strategies (Zakamulin 2014).

The remainder of this paper is structured as follows. In Section 4.2, the formal relationship between beta and future market risk premium is derived, and the economic idea and concept of why beta dispersion is a measure of market vulnerability are developed. Section 4.3 describes the data and basic methodology. Section 4.4 presents the empirical results, 
consisting of the decomposition of returns, descriptive statistics, statistical analyses, and market timing strategies and their performance. Section 4.5 concludes the paper.

\subsection{Systematic Risk and Market Return}

\subsubsection{Stochastic Beta and Market Timing}

The economic idea of this study is based on and motivated by the conditional CAPM, which suggests a time-varying beta coefficient conditional on the current information set. The use of a variable beta is supported by strong evidence in the empirical literature that beta is in fact time-varying (Faff et al. 2000; Andersen et al. 2005; Jostova and Philipov 2005; Reeves and Wu 2013; Bali et al. 2016; Hollstein and Prokopczuk 2016). Consequently, the conditional CAPM explains expected returns like the traditional CAPM but more effectively by loosening the one period perspective (Hansen and Richard 1987; Ferson and Harvey 1993; Jagannathan and Wang 1996; Wang 2003; Lewellen and Nagel 2006).

To establish the relationship between beta and the market return, I start with a general formulation of the conditional CAPM, where beta is time-dependent, following Jagannathan and Wang (1996). The expected return of an asset $E\left(R_{i, t+1} \mid I_{t}\right)$ at time $t+1$ under the information set $I_{t}$ is given by

$$
E\left(R_{i, t+1} \mid I_{t}\right)=\gamma_{0, t}+\beta_{i, t} \gamma_{m, t}, \quad \text { with } \quad \beta_{i, t}=\frac{\operatorname{Cov}\left(R_{i, t+1}, R_{M, t+1} \mid I_{t}\right)}{\operatorname{Var}\left(R_{M, t+1} \mid I_{t}\right)}
$$

where $\gamma_{0, t}$ is the conditional expected return on a zero-beta portfolio, $\gamma_{m, t}$ is the conditional market risk premium, $\beta_{i, t}$ is the conditional beta of asset $i$ under the information set $I_{t}$, and $R_{M, t+1}$ is the return of the market portfolio in $t+1$. By taking the unconditional expectations on both sides of Equation 4.1, the unconditional expected return of asset $i$ is

$$
E\left(R_{i, t+1}\right)=E\left(\gamma_{0, t}\right)+E\left(\beta_{i, t}\right) E\left(\gamma_{m, t}\right)+\operatorname{Cov}\left(\beta_{i, t}, \gamma_{m, t}\right)
$$

The unconditional return of asset $i$ is represented by the sum of the expected return of the zero-beta portfolio, the product of the expected beta of the asset and the expected return 
of the market, and the covariance between these two. The covariance term in Equation 4.2 can be further decomposed by splitting the $\beta_{i, t}$ in the previous $\beta_{i, t-1}$ and the actual change $\left(\beta_{i, t}-\beta_{i, t-1}\right)$. Hence,

$$
\operatorname{Cov}\left(\beta_{i, t}, \gamma_{m, t}\right)=\underbrace{\operatorname{Cov}\left(\beta_{i, t}-\beta_{i, t-1}, \gamma_{m, t}\right)}_{\text {natural hedge component }}+\underbrace{\operatorname{Cov}\left(\beta_{i, t-1}, \gamma_{m, t}\right)}_{\text {market timing component }} .
$$

The first part of Equation 4.3 is called natural hedge component and can be interpreted as an insurance of the portfolio value. The market timing component is defined by the second term of this split covariance. This part of the covariance shows the linkage between the previous, or ex-ante, beta of an asset in $t-1$ and the ex-post market risk premium in t. If it is positive, then a high ex-ante beta will be associated with a positive market risk premium in the following period. If it is negative, then high betas will be an indicator of a negative market risk premium and a potential market decline. Therefore, if this covariance is significantly different from zero, then ex-ante betas might indicate the future market return.

The assets or portfolios for which the market timing component can be substantially different from zero must be carefully considered, and hence must be studied empirically. The identification of a portfolio whose composition depicts the economic idea of why stock betas can predict the future market return seems to be a reasonable approach. Such a portfolio would be expected to have a measurable market timing component. The economic concept of the beta dispersion, introduced in detail in Section 4.2.2, can provide this economic foundation and suggests such a deliberated portfolio composition. A portfolio composed of stocks capturing the beta dispersion of a market should have an ex-ante beta that can forecast potential market declines. The market timing component of such a portfolio should be significantly negative and, hence, the beta of the portfolio should be able to indicate negative market returns. 


\subsubsection{Beta Dispersion as a Measure of Market Vulnerability}

The spread between high betas and low betas on a market is defined as beta dispersion. In other words, beta dispersion refers to the deviation of the betas' distribution from its mean at a particular point in time. By definition, the market beta equals one, and, accordingly, betas of all stocks in that market have to group around this weighted mean. Until now, not much attention has been paid to how widespread or concentrated the individual betas are and whether this has any impact on the stability of the market and, consequently, on the market return.

To illustrate the economic idea behind the beta dispersion as a measure for market vulnerability, two markets that are completely identical, except for their specific beta dispersion, can be considered. On market A, the beta dispersion is narrow, that is, the betas of all the stocks are clotted closely around one. Contrarily, on market B, the beta dispersion is large, and the betas are widespread, meaning that some stocks have beta values close to zero and some have extremely high betas. Now, both markets experience an identical systematic exogenous shock. All the stocks in market A should react very similarly because the companies are affected rather alike by the shock. Hence, this should be reflected in the stock prices as the shock hits all the stocks nearly equally strongly owing to their more or less equal betas. On market B, stocks with a low beta, close to zero, hardly react to the systematic shock, because the companies are hardly affected in their business operations by the shock. Contrarily, stocks with a high beta react intensely, indicating that for such companies the systematic shock might threaten their businesses in a way which jeopardizes their economic survival. This increases the risk of financial distress in market B. Since most of the firms in a market are interconnected, the collapse of endangered high-beta firms might spill over to those firms that are strongly connected to them. The spillover spreads via all possible transmission channels. This includes physical, legal, and intangible ways, such as loss of confidence. These contagion effects trigger an endogenous second round systematic shock (the risen level of distress risk) on market B and can lead to an overall market downturn if this spillover, or better yet, cascading effect 
proceeds. This makes beta dispersion an indicator of the vulnerability of the market and represents systemic risk characteristics of the market.

In summary, the larger the beta dispersion of a specific market, the higher the likelihood of a subsequent second-round shock following an initial exogenous shock. Therefore, a market with a high beta dispersion is more likely to be financially unstable compared to a market with a relatively narrower beta dispersion. If the beta dispersion is high, the market is especially endangered towards systematic shocks. With a high beta dispersion, the probability rises that a moderate systematic shock evolves and leads to a severe market decline due to the cascading effect. For example, during the subprime crisis, the financial institutions often exhibited extremely high betas. Even a moderate market shock can cause serious problems for such high-beta firms and affect the entire financial system because of the interconnectedness of financial institutions. Large beta dispersion and the concentration of high-beta companies within a specific industrial sector eventually lead to a severe overall market downturn when compared to a market with a more moderate beta dispersion. In this context, the beta dispersion can be considered a measure for the vulnerability of the market stability. Contrarily, such a strong downturn would be less likely if there were no stocks with extremely high betas or the high-beta stocks were less industrially concentrated and interconnected. This would make the cascading effect less pronounced. If this conclusion applies, then the beta dispersion would not only be a measure for the vulnerability of a market and a predictor of the magnitude of future market downturns but also a promising approach of extending systemic risk measures.

Based on this economic explanation, beta dispersion can be considered an explicit measure for the probability and extent of market declines. This is supported by the fundamental connection between stochastic ex-ante beta and ex-post market return, as derived in Section 4.2.1. Therefore, a portfolio mimicking the beta dispersion should be highly appropriate as a portfolio designed for quantifying the market timing component, which is expected to be significantly measurable. Nevertheless, it would be crucial to reflect how the beta dispersion can be measured to depict the economic idea and not waste any information. The first notion to measure the spread between high and low betas on the market is to compare the tails of the beta distribution in terms of extreme quantiles. This facilitates 
easy implementation of portfolio composition for testing the existence of the market timing component. Besides this, a reasonable definition of beta dispersion can be derived based on the return dispersion, which is the cross-sectional deviation of stock returns from their value-weighted mean. It quantifies the heterogeneity of stock returns at a certain point in time. In this sense, a beta dispersion will be represented by the cross-sectional deviation of betas from their mean and capture how heterogeneous the betas are at a certain point in time. Both definitions of beta dispersion combine a cross-sectional and a time dimension of market properties and aggregate them into a single measure of market vulnerability. Each measure emphasizes a specific feature of beta dispersion. While the first approach concentrates on the stocks that are most endangered, following the economic idea of the beta dispersion, the second approach considers all the stocks in a market and, therefore, all possible information. Hence, both measures are studied in the empirical section.

The beta dispersion can be clearly differentiated from the return dispersion in two ways. First, the beta dispersion is suitable as an explicit measure for market vulnerability and it focuses on the systematic component of stocks. The return dispersion can be understood as a model-free measure of market volatility. Angelidis et al. (2015) show that return dispersion can predict the expected market return as it is an alternative specification of market risk. The beta dispersion has a different economic idea and should explicitly predict the extent of market downturns. The heterogeneous behavior of companies and their business operations is accompanied with different sensitivities to systematic shocks, which is highlighted by this definition. The beta dispersion is better suited to measure this heterogeneity in stock returns because the beta standardizes the diverse return movements and makes them comparable in terms of sensitivity toward systematic risk. It must be noted that a large return dispersion can be caused by idiosyncratic risk and, thus, can be much less informative about market vulnerability, which is of special interest in this study. The idea that a cascading effect pushes the market into a crisis seems to be more straightforward when reducing the source of risk to systematic shocks. 


\subsection{Data and Methodology}

The empirical analyses on beta dispersion are based on daily stock prices and market values of the S\&P 500 Index and its constituents from April 1964 to December 2016. The data source for the stock data is the Wharton Research Data Service (WRDS), and the period is constrained by the availability of detailed information on the constituents of the S\&P 500 . In addition, the industry of each stock in the S\&P 500 Index is needed. To classify the stocks, the S\&P standard sector definition is used to ensure that the stocks are allocated to ten different, broad sectors including consumer discretionary, consumer staples, energy, financials, healthcare, industrials, materials, real estate, technology, and utilities. The focus on 500 highly liquid stocks is advantageous as it ensures the availability of the daily price data, which, in turn, ensures an appropriate beta estimation. Moreover, the availability of highly liquid exchange-traded funds (ETFs) on the S\&P 500 Index facilitate easy trading the market, which will be crucial to the market timing strategy at a later stage. This also ensures the implementation of the timing strategies at relatively low transaction costs. As the risk-free interest rate, the 1-month T-bill rate from Kenneth French's website is used. For additional analysis, the cay factor (Lettau and Ludvigson 2001), which is obtained from Martin Lettau's website, dividend yield of the S\&P 500 Index (from WRDS), and volatility index (VIX) (accessed via Datastream) are needed. Additional variables are calculated based on the described data. ${ }^{1}$

Betas are estimated with different rolling windows from daily returns over $3,6,12$, and 36 months. These window lengths ensure that sufficient observations are available to estimate reliable betas. The estimates are calculated at the end of each month starting in April 1964 and ending in December 2016. Based on these betas, the two introduced measures of beta dispersion are calculated. The first approach measures the current difference between the mean beta of the high-beta quantile $\bar{\beta}_{t}^{\text {High }}$ (90\%-quantile) and the mean beta of the low-beta quantile $\bar{\beta}_{t}^{\text {Low }}$ (10\%-quantile) of all constituents of the S\&P 500 Index ranked by their beta (compare Equation 4.4):

\footnotetext{
${ }^{1} \mathrm{~A}$ precise description of the additional variables and their calculation can be found in the Appendix 4.A.1.
} 


$$
Q B D_{t}=\bar{\beta}_{t}^{\text {High }}-\bar{\beta}_{t}^{\text {Low }} .
$$

The second approach measures the value-weighted as well as the equal-weighted square root squared deviation of all betas from their mean, which is formally defined in Equation 4.5:

$$
B D_{t}=\sqrt{\sum_{i=1}^{n}\left(w_{i, t} \cdot \beta_{i, t}-\sum_{i=1}^{n}\left(w_{i, t} \cdot \beta_{i, t}\right)\right)^{2}} .
$$

In this analysis $\beta_{i, t}$ is the estimated beta of stock $i$ and $w_{i, t}$ is the value- or equal-weighted share of stock $i$ in the index. The value-weighted version gives additional impact to the large stocks in the index. However, large firm-size does not automatically indicate that the company is highly interconnected with other firms, which is vital to the idea of beta dispersion. The equal-weighted version of the Equation 4.5 only focuses on the extent to which the betas are widespread, independently of other firm characteristics.

\subsection{Empirical Implementation and Results}

\subsubsection{Empirical Decomposition of Returns}

First, it must be ascertained if the market timing component from Equation 4.3 is statistically significant different from zero and provides any informational content. Portfolios that mimic the beta dispersion are used to quantify the covariance of the ex-ante beta and ex-post market returns empirically. All the stocks are ranked monthly by their beta, estimated with different periods of daily data, and sorted in $10 \%$-quantile portfolios. ${ }^{2} \mathrm{~A}$ portfolio that is composed of a long position in the portfolio with the highest mean beta and a short position in the one with the lowest mean beta is studied as this depicts the first definition of the beta dispersion, compared to Equation 4.4. For a more detailed analysis, the market timing component is also quantified for the portfolio with the highest and the

\footnotetext{
${ }^{2}$ The results also hold if the stocks are sorted in 5\%-quantiles.
} 
lowest mean beta solely. The results for all the three portfolio compositions are presented in Table 4.1.

\section{Table 4.1: Decomposition of Returns: Market Timing Component}

\begin{tabular}{|c|c|c|c|c|}
\hline Ex-ante & Ex-post & $\begin{array}{l}\text { Low-beta } \\
\text { Portfolio }\end{array}$ & $\begin{array}{c}\text { High-beta } \\
\text { Portfolio }\end{array}$ & $\begin{array}{c}\text { Beta-dispersion } \\
\text { Portfolio }\end{array}$ \\
\hline 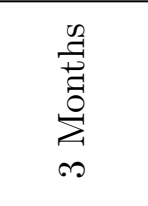 & $\begin{array}{l}1 \text { Month } \\
3 \text { Months } \\
6 \text { Months } \\
12 \text { Months }\end{array}$ & $\begin{array}{l}0.0066^{*} \\
0.0063^{*} \\
0.0060^{*} \\
0.0059\end{array}$ & $\begin{array}{l}-0.0175^{* * *} \\
-0.0144^{* * *} \\
-0.0104^{* * *} \\
-0.0089^{* * *}\end{array}$ & $\begin{array}{l}-0.0241^{* * *} \\
-0.0207^{* * *} \\
-0.0163^{* * *} \\
-0.0148^{* * *}\end{array}$ \\
\hline $\begin{array}{l}\frac{n}{2} \\
\text { 节 } \\
\sum_{0}^{1} \\
0\end{array}$ & $\begin{array}{l}1 \text { Month } \\
3 \text { Months } \\
6 \text { Months } \\
12 \text { Months }\end{array}$ & $\begin{array}{l}0.0049 \\
0.0051 \\
0.0050 \\
0.0052\end{array}$ & $\begin{array}{l}-0.0129^{* * *} \\
-0.0111^{* * *} \\
-0.0084^{* * *} \\
-0.0069^{* * *}\end{array}$ & $\begin{array}{l}-0.0179^{* * *} \\
-0.0162^{* * *} \\
-0.0134^{* * *} \\
-0.0121^{* * *}\end{array}$ \\
\hline 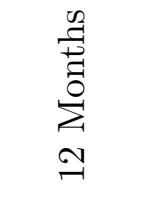 & $\begin{array}{l}1 \text { Month } \\
3 \text { Months } \\
6 \text { Months } \\
12 \text { Months }\end{array}$ & $\begin{array}{l}0.0051 \\
0.0051 \\
0.0050 \\
0.0049\end{array}$ & $\begin{array}{l}-0.0114^{* * *} \\
-0.0093^{* * *} \\
-0.0071^{* * *} \\
-0.0047^{* * *}\end{array}$ & $\begin{array}{l}-0.0164^{* * *} \\
-0.0144^{* * *} \\
-0.0121^{* * *} \\
-0.0096^{* * *}\end{array}$ \\
\hline $\begin{array}{l}\sum_{0}^{n} \\
\sum_{0}^{0} \\
0 \\
0\end{array}$ & $\begin{array}{l}1 \text { Month } \\
3 \text { Months } \\
6 \text { Months } \\
12 \text { Months }\end{array}$ & $\begin{array}{l}0.0047 \\
0.0048 \\
0.0046 \\
0.0042\end{array}$ & $\begin{array}{l}-0.0049^{* *} \\
-0.0040^{* * *} \\
-0.0031^{* * *} \\
-0.0017^{* * *}\end{array}$ & $\begin{array}{l}-0.0096^{* * *} \\
-0.0088^{* * *} \\
-0.0077^{* * *} \\
-0.0059^{* * *}\end{array}$ \\
\hline
\end{tabular}

Note: Table 4.1 shows the market timing component quantified for portfolios composed of the constituents of the S\&P 500 and grouped by low-beta stocks (10\%-quantile), high-beta stocks (90\%-quantile), and the difference between the two to mimic the idea of the beta dispersion. Ex-ante betas are estimated for 3, 6, 12 , and 36 months and the ex-post market return is calculated for 1, 3, 6, and 12 months from the daily price data of the S\&P 500. This decomposition is based on Equation 4.3. Accordingly, the market timing component comprises the covariance between the estimated ex-ante beta and the ex-post market return. The covariance is calculated from daily data for the whole investigation period (April 1964 to December 2016). All the values are annualized. Significance level: *** $0.01,{ }^{* *} 0.05$, and ${ }^{*} 0.1$.

Table 4.1 shows the values of the market timing component for a beta-dispersion portfolio, mimicking the economic idea of beta dispersion, a low-beta portfolio and a high-beta portfolio. The calculation of the market timing component follows Equation 4.3 and all values are annualized to make the covariance terms comparable. Almost all observations are significant at a $1 \%$ level for the beta-dispersion portfolio and high-beta portfolio over the different estimation and holding periods. The shorter the estimation period of the beta and the shorter the period for the market return calculation, the more negative is the covariance for the beta dispersion and high-beta portfolio. The beta-dispersion portfolio 
results in the most pronounced and significant values for the market timing component. The greater the difference between the low and high betas in a market, the more the market tends to go down in the next period. The market timing component for the low-beta portfolio is rather small and close to zero. Nevertheless, in the economic sense, it means that if the beta of a low-beta portfolio in the market is rather low, then the market tends to decline in the next period. Contrarily, concerning a high-beta portfolio, only negative values are discovered, with significant values up to $-1.75 \%$. This indicates that unusually high betas in a market lead to an expected decline in market returns. Taken together, these two findings indicate the origin of the high negative values of the market timing component of the beta-dispersion portfolio. Mainly, the high-beta stocks drive the beta dispersion and its market timing ability. Nevertheless, the low-beta stocks do not contradict this finding, but support it. However, this support is not statistically significant. The results suggest that the market timing ability is more pronounced for the beta-dispersion portfolio than for only one of the two portfolios. This finding provides the first evidence of the applicability of the concept of beta dispersion as the predictor of the magnitude and probability of market declines.

In a nutshell, the existence of timing ability of the beta can be confirmed for certain portfolios. The market timing component has different signs for low-beta and high-beta portfolios which ensures that it matches the idea of beta dispersion. The beta-dispersion portfolio carries the combined information. The empirical findings allow to conclude that the market timing component exists for certain portfolios. This supports the hypothesis that a beta can contain information about the future market return, and high levels of beta dispersion can foreshadow market downturns. Given these promising results in Table 4.1 together with the economic interpretation of beta dispersion, it is natural to study the effects in detail.

\subsubsection{Empirical Description of the Beta Dispersion}

The following subsections conduct in-depth analyses of beta dispersion and the applicability of the economic idea. Section 4.4.1 shows that a portfolio the mimicking beta dispersion 
has a measurable and significantly negative market timing component. Therefore, it can be concluded that a high beta dispersion can indicate a negative future market return. This section uses different descriptive approaches to confirm this initial piece of evidence and to analyze the suitability of the economic concept of beta dispersion. A visual analysis of beta dispersion and market performance can give a basic understanding of the underlying predictive relationship. In order to visualize the economic idea, Figure 4.1 shows the price development of the S\&P 500 Index, representing the market in this study, and the beta dispersion in this market.

\section{Figure 4.1: Time Series of Beta Dispersion and S\&P 500 Index Level}

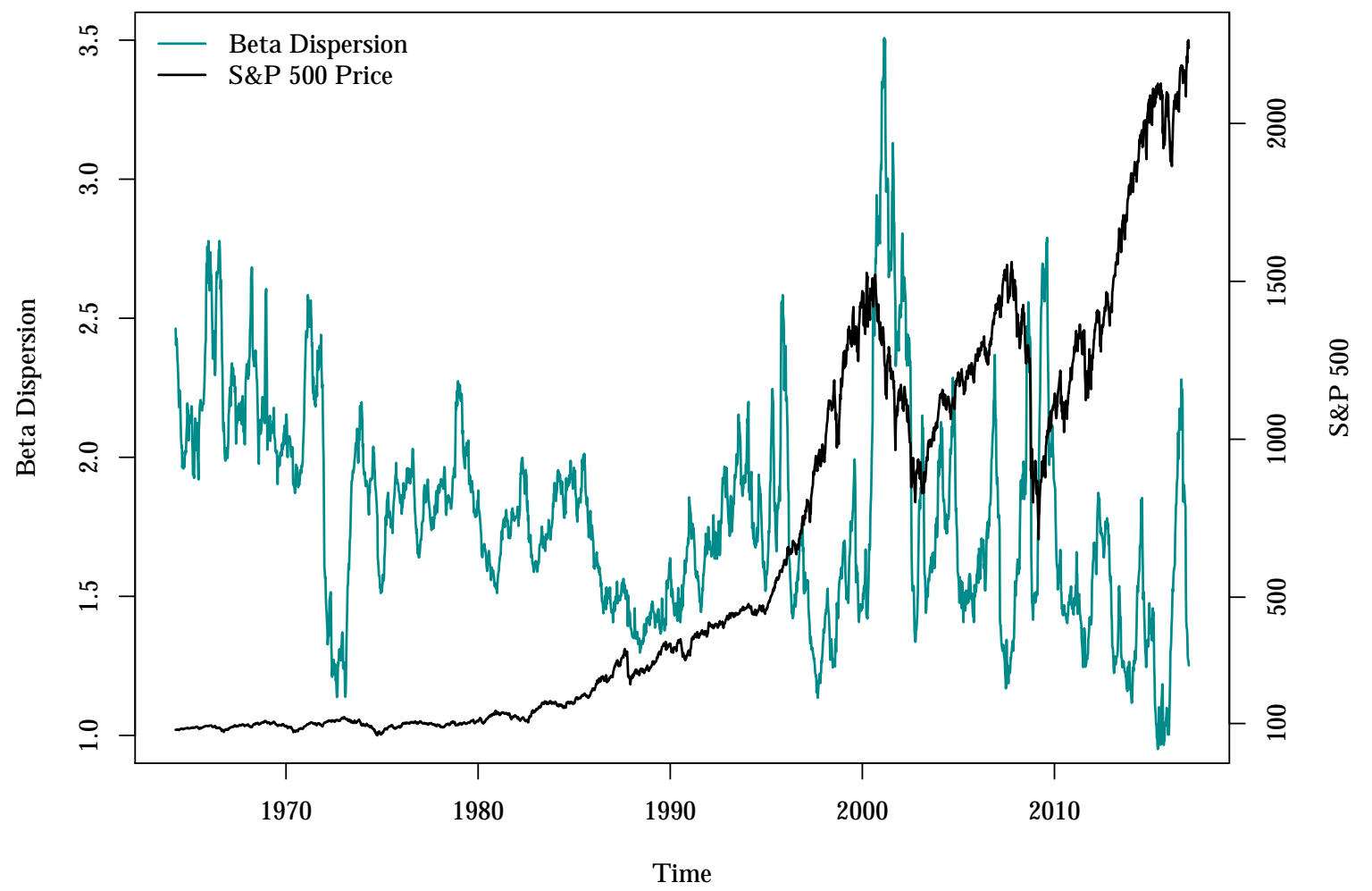

Note: Figure 4.1 shows the time series of beta dispersion and the level of the S\&P 500 Index from the period April 1964 to December 2016. The beta dispersion is calculated as the difference between the 90\%and 10\%-quantile of the beta-sorted constituents of the S\&P 500 Index.

In this figure, the beta dispersion is calculated with the quantile approach, following Equation 4.4. This figure supports the hypothesis that beta dispersion can be interpreted as a vulnerability measure and the economic idea seems to hold. The beta dispersion had been on an exceptionally high level on previous occasions of major market downturns. This 
was particularly noticeable around the dotcom bubble in 2000 and was less pronounced from 2008 onwards. The examination of separate time series of the $90 \%$ quantile and the $10 \%$ quantile of beta distribution leads to the conclusion that the variation of the beta dispersion is mainly caused by variations in the high-beta quantile. The variation of the $10 \%$ quantile (low-beta quantile) is relatively narrow and stable over the sampled period. Contrarily, the variability of the $90 \%$ quantile (high-beta quantile) is remarkable. This is in line with the results in Section 4.4.1, where the high-beta portfolio drives the beta-dispersion portfolio. This also corresponds to the economic concept as the cascading effect is caused by the stumbling of the high-beta companies.

The effect of a second endogenous shock should be more pronounced if most of the stocks with high betas are operating within the same industrial sector. A closer look is now taken at the high-beta stocks. If the industry-specific concentration in the high-beta quantile coincides with a high beta dispersion, then the cascading effect on other companies in different industrial sectors would be intensified as there will be an increase in the bankruptcy risk of not only one company but also of the whole sector. Figure 4.2 shows that the industry-specific concentration of high-beta stocks increases prior to market downturns and also slightly in advance of increases in the beta dispersion. This can be seen in the dotcom bubble of 2000 and the subprime crisis of 2008. The concentration is measured as the maximum absolute number of stocks from the same sector included in the high-beta quantile at a certain point in time. 
Figure 4.2: Beta Dispersion and the Concentration of Stocks in One Sector

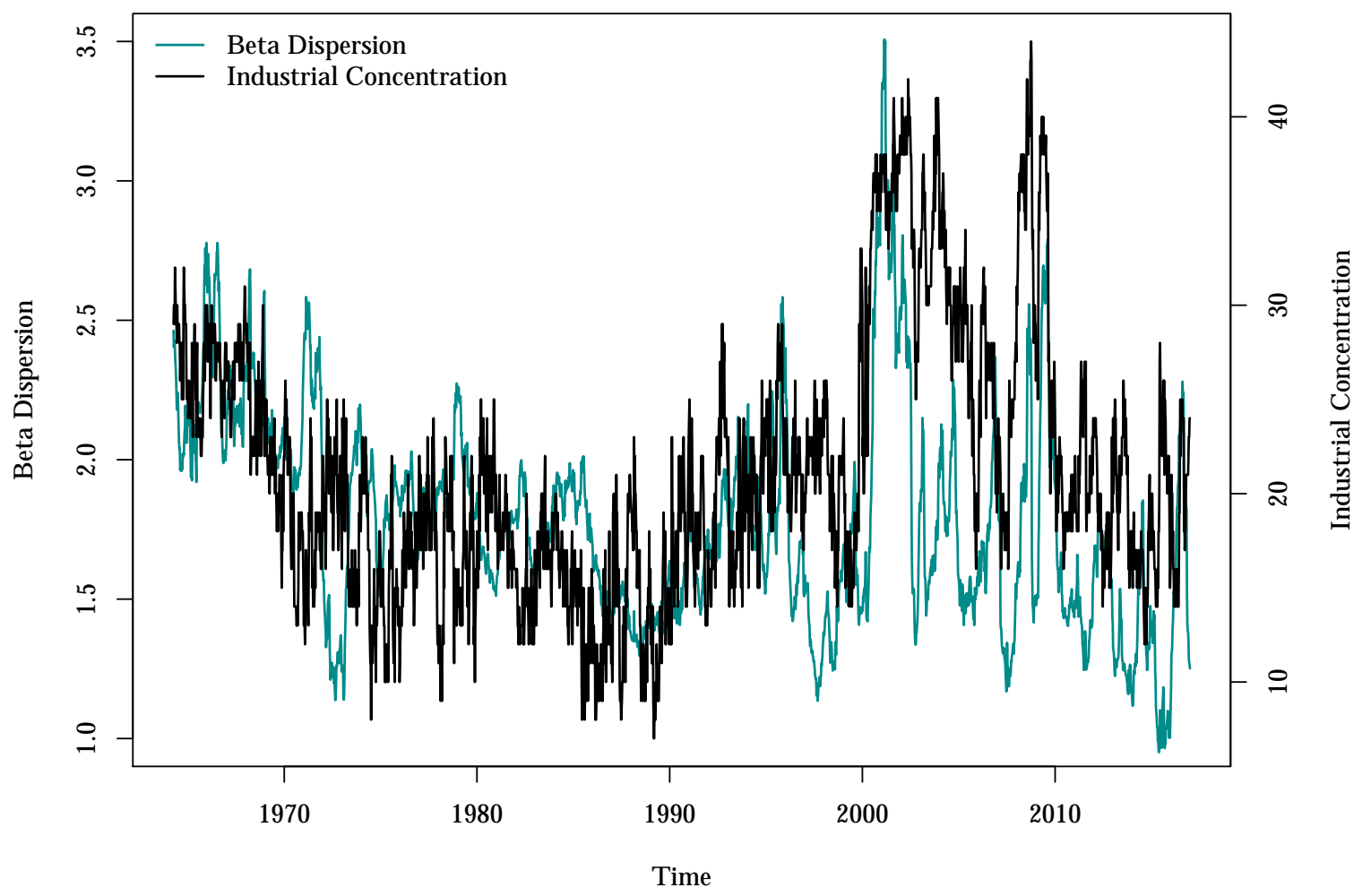

Note: Figure 4.2 shows the time series of beta dispersion and the concentration of the stocks in one specific sector in the high-beta decile from April 1964 to December 2016. The concentration comprises the absolute amount of stocks in the quantile that stem from the same sector. The S\&P standard sector definition is used for this classification to ensure that the stocks are allocated to 10 different sectors (consumer discretionary, consumer staples, energy, financials, healthcare, industrials, materials, real estate, technology, and utilities). The maximum concentration is 50 stocks due to the construction of deciles.

In 2001, a maximum of 45 out of 50 stocks belonged to the same industrial sector. The sectors in which the stocks were concentrated were those that were hit by the crisis first. Contrary to the high-beta quantile, the industrial concentration of stocks in all other quantiles is rather stable and, by far, not as highly concentrated as in the high-beta quantile. The imbalance was due to the technology stocks before the dotcom bubble and financial sector stocks before the subprime crisis. These findings corroborate the hypothesis why the second endogenous shock is extensive if an initial systematic exogenous shock simultaneously and strongly hits a whole industrial sector. A high beta dispersion combined with a high industrial concentration seems to be a strong measure for the vulnerability of a market. This means systematic shocks cannot be absorbed easily by this market. 


\subsubsection{Predicting Market Returns - In-sample Evaluation}

Predictive regressions are typically conducted to investigate and quantify an assumed predictive relationship. The dependent variable, here the log excess return of the market $R_{M, t}$, is regressed on a lagged explanatory or better predictive variable $P_{t-1}$. The general form of this linear regression is shown in Equation 4.6 below:

$$
R_{M, t}=\alpha+\sum \beta_{P_{i}} P_{i, t-1}+\varepsilon_{t}
$$

with $\beta_{P_{i}}$ is the coefficient of predictive variable $i$, and $\varepsilon_{t}$ being the disturbance term of the regression. This form of analysis is also referred to as in-sample prediction because the whole data sample is used to estimate the regression parameters. Predictive regressions face statistical particularities that have to be respected. If the dependent and predictive variables are both calculated from the same data (e.g., asset prices), then the autocorrelation in the $\varepsilon_{t}$ can be induced because the data point at the split between $t-1$ and $t$ is included in the calculation of both the variables (Stambaugh 1999). Another potential source of artificial autocorrelation and, thus, biased estimators, is a dependent variable calculated over more than one period. These problems can be solved by bootstrapping and simulation of critical test statistics (Bollerslev et al. 2009, 2014) and by following the correction proposed by Britten-Jones et al. (2011). To address the first mentioned source of artificial autocorrelation, it is ensured that there is no overlap between the data used to calculate the dependent and the predictive variables. In addition, by a transformation of the explanatory variables matrix, the autocorrelation in the error terms, which is caused by overlapping periods, can be removed. This correction for artificial autocorrelation of Britten-Jones et al. (2011) is used for all the following regressions. In addition,

To test whether the beta dispersion is informative for future market movements, predictive regressions are run to explain the log excess return of the market $\left(R_{M, t}\right)$ at time t for the next $1,3,6$, and 12 months. To begin with, the lagged measure of the beta dispersion $Q B D_{t-1}$, respectively, the value-weighted and equal-weighted $B D_{t-1}$, as defined in Equation 4.4 and 
in Equation 4.5, respectively, is the only explanatory variable. The predictive regression follows the below-mentioned form:

$$
R_{M, t}=\alpha+\beta \cdot(Q) B D_{t-1}+\varepsilon_{t} .
$$

The results of these predictive regressions with the equal-weighted beta dispersion $\left(B D_{E W}\right)$ as explanatory variable for the log excess return of the market are given in Table $4.2 .^{3}$ Panel A presents the results for the 1-month market return, Panel B for the 3-month market return, Panel $\mathrm{C}$ for the 6-month market return, and Panel D for the 12-month market return.

\footnotetext{
${ }^{3}$ The results of the predictive regression with the value-weighted beta dispersion $\left(B D_{V W}\right)$ and the beta dispersion based on quantiles $(Q B D)$ are given in the Appendix 4.A.2, because they hardly give any additional insights.
} 
Table 4.2: Linear Predictive Regressions with $B D_{E W}$

Panel A: 1-Month Market Returns

\begin{tabular}{lcccc}
\hline & $B D_{E W}^{3 M}$ & $B D_{E W}^{6 M}$ & $B D_{E W}^{12 M}$ & \multicolumn{1}{c}{$B D_{E W}^{36 M}$} \\
\hline Constant & $\mathbf{0 . 0 1 3 6}$ & $\mathbf{0 . 0 1 4 7}$ & $\mathbf{0 . 0 1 5 5}$ & $\mathbf{0 . 0 1 5 4}$ \\
& $(0.0004)$ & $(0.0001)$ & $(0.0000)$ & $(0.0007)$ \\
Coefficient & $\mathbf{- 0 . 0 2 1 2}$ & $\mathbf{- 0 . 0 3 0 9}$ & $\mathbf{- 0 . 0 3 9 9}$ & $\mathbf{- 0 . 0 4 7 7}$ \\
& $(0.0222)$ & $(0.0115)$ & $(0.0201)$ & $(0.0157)$ \\
$R_{a d j}^{2}$ & 0.0078 & 0.0092 & 0.0115 & 0.0083 \\
\hline
\end{tabular}

Panel B: 3-Month Market Returns

\begin{tabular}{|c|c|c|c|c|}
\hline & $B D_{E W}^{3 M}$ & $B D_{E W}^{6 M}$ & $B D_{E W}^{12 M}$ & $B D_{E W}^{36 M}$ \\
\hline Constant & $\begin{array}{c}\mathbf{0 . 0 5 3 9} \\
(0.0000)\end{array}$ & $\begin{array}{c}\mathbf{0 . 0 5 9 0} \\
(0.0001)\end{array}$ & $\begin{array}{c}\mathbf{0 . 0 5 8 5} \\
(0.0002)\end{array}$ & $\begin{array}{l}\mathbf{0 . 0 6 0 7} \\
(0.0007)\end{array}$ \\
\hline Coefficient & $\begin{array}{r}-\mathbf{0 . 0 8 3 8} \\
(0.0033)\end{array}$ & $\begin{array}{r}-\mathbf{0 . 1 2 4 8} \\
(0.0049)\end{array}$ & $\begin{array}{r}-\mathbf{0 . 1 4 6 7} \\
(0.0068)\end{array}$ & $\begin{array}{r}-\mathbf{0 . 1 8 6 8} \\
(0.0159)\end{array}$ \\
\hline$R_{a d j}^{2}$ & 0.0228 & 0.0234 & 0.0230 & 0.0210 \\
\hline
\end{tabular}

Panel C: 6-Month Market Returns

\begin{tabular}{lcccc}
\hline & $B D_{E W}^{3 M}$ & $B D_{E W}^{6 M}$ & $B D_{E W}^{12 M}$ & $B D_{E W}^{36 M}$ \\
\hline Constant & $\mathbf{0 . 0 9 4 3}$ & $\mathbf{0 . 1 0 5 5}$ & $\mathbf{0 . 1 0 1 0}$ & $\mathbf{0 . 1 0 1 9}$ \\
& $(0.0000)$ & $(0.0000)$ & $(0.0003)$ & $(0.0016)$ \\
Coefficient & $\begin{array}{r}\mathbf{- 0 . 1 4 6 8} \\
(0.0014)\end{array}$ & $\begin{array}{c}\mathbf{- 0 . 2 2 6 6} \\
(0.0019)\end{array}$ & $\begin{array}{c}\mathbf{- 0 . 2 5 1 8} \\
(0.0120)\end{array}$ & $\begin{array}{c}\mathbf{- 0 . 3 0 6 6} \\
(0.0279)\end{array}$ \\
$R_{a d j}^{2}$ & 0.0237 & 0.0252 & 0.0231 & 0.0198 \\
\hline
\end{tabular}

Panel D: 12-Month Market Returns

\begin{tabular}{lcccc}
\hline & $B D_{E W}^{3 M}$ & $B D_{E W}^{6 M}$ & $B D_{E W}^{12 M}$ & $B D_{E W}^{36 M}$ \\
\hline Constant & $\mathbf{0 . 1 5 4 7}$ & $\mathbf{0 . 1 7 0 6}$ & $\mathbf{0 . 1 6 7 7}$ & $\mathbf{0 . 1 6 2 3}$ \\
& $(0.0000)$ & $(0.0003)$ & $(0.0010)$ & $(0.0068)$ \\
Coefficient & $\mathbf{- 0 . 2 2 1 6}$ & $\mathbf{- 0 . 3 3 9 0}$ & $\mathbf{- 0 . 3 8 9 8}$ & $\mathbf{- 0 . 4 4 2 4}$ \\
& $(0.0001)$ & $(0.0107)$ & $(0.0248)$ & $(0.0841)$ \\
$R_{a d j}^{2}$ & 0.0204 & 0.0214 & 0.0204 & 0.0164 \\
\hline
\end{tabular}

Note: Table 4.2 shows the results of the predictive regressions, with the beta dispersion as independent variable and the 1-, 3-, 6-, and 12-month log excess return of the S\&P 500 Index (Panel A, Panel B, Panel $\mathrm{C}$, and Panel D) as the dependent variable. BD is the cross-sectional equal-weighted standard deviation of the individual stocks' betas (compare Equation 4.5). Beta is estimated from daily returns over a period of $3,6,12$, and 36 months. The adjusted $R_{a d j}^{2}$ of the predictive regressions are given in the last row of the table. Overlapping periods of the dependent variable are addressed by using the correction proposed by Britten-Jones et al. (2011). The calculations also use the Newey-West estimator with corresponding lags to account for heteroscedasticity and autocorrelation in the residuals. The p-value is given in parenthesis for every coefficient. Coefficients that are significant at least at a $10 \%$ level are printed in boldface. 
All specifications of the beta dispersion generate significant results at least at a $10 \%$ level, which is generally much higher. All coefficients have a negative sign, as expected, following the idea of beta dispersion as a vulnerability measure. This is in line with the results of Section 4.4.1. It shows that a very high beta dispersion in the estimation period tends to be associated with a negative market return in the following period. The shorter the estimation period of the betas, the more significant the coefficient in the predictive regression. The in-sample $R_{a d j}^{2}$ are the highest in the estimation of beta, with 6 months of daily data. For this estimation period, the calculated betas are relatively stable as around 125 data points are used for the estimation, and hence short-term disturbances disappear during this period. All conclusions drawn from Table 4.2 are applicable for the other two measures $\left(B D_{V W}\right.$ and $\left.Q B D\right)$. Taken together, the $B D_{E W}$ slightly outperform $B D_{V W}$ and $Q B D$ for the long-horizon prediction. This finding is not surprising as the $B D_{E W}$, on the one hand, neglects firm characteristics, for example size, and, on the other hand, includes information of all the stocks in the market, while the $Q B D$ measure only focuses on the tails of the beta distribution. Owing to this similarity in results, only the results of the equal-weighted BD measure are reported in the following section. ${ }^{4}$

Beside the correction of Britten-Jones et al. (2011) for overlapping periods, the simple predictive regressions are run with non-overlapping periods. Therefore, the observations are deconstructed to observation time series with non-overlapping market returns. For the 3-month market return, the regressions are run only every fourth month to ensure that there is no overlap in the 3 -month returns. The starting point of this deconstruction is shifted over time to ensure that all observations are included, but in different regressions. The same is done for the 6- and 12-month returns.

\footnotetext{
${ }^{4}$ All analyses also apply for the value-weighted BD and the QBD measure. As they do not deliver additional insights, the results are not displayed in this study, but are available upon request.
} 
Table 4.3: Predictive Regressions with Non-overlapping Periods

Panel A: 3-Month Market Returns

\begin{tabular}{|c|c|c|c|c|}
\hline & $B D_{E W}^{3 M}$ & $B D_{E W}^{6 M}$ & $B D_{E W}^{12 M}$ & $B D_{E W}^{36 M}$ \\
\hline Constant & $\begin{array}{l}\mathbf{0 . 0 6 0 4} \\
(0.0000)\end{array}$ & $\begin{array}{l}\mathbf{0 . 0 5 3 4} \\
(0.0000)\end{array}$ & $\begin{array}{l}\mathbf{0 . 0 4 5 9} \\
(0.0005)\end{array}$ & $\begin{array}{l}\mathbf{0 . 0 4 8 5} \\
(0.0003)\end{array}$ \\
\hline Coefficient & $\begin{array}{r}-\mathbf{0 . 1 2 2 9} \\
(0.0003)\end{array}$ & $\begin{array}{r}-\mathbf{0 . 1 3 0 6} \\
(0.0001)\end{array}$ & $\begin{array}{r}-\mathbf{0 . 1 3 0 6} \\
(0.0004)\end{array}$ & $\begin{array}{r}-\mathbf{0 . 1 6 9 8} \\
(0.0056)\end{array}$ \\
\hline$R_{a d j}^{2}$ & 0.0734 & 0.0544 & 0.0357 & 0.0303 \\
\hline
\end{tabular}

Panel B: 6-Month Market Returns

\begin{tabular}{lcccc}
\hline & $B D_{E W}^{3 M}$ & $B D_{E W}^{6 M}$ & $B D_{E W}^{12 M}$ & $B D_{E W}^{36 M}$ \\
\hline Constant & $\mathbf{0 . 0 9 2 8}$ & $\mathbf{0 . 0 9 2 3}$ & $\mathbf{0 . 0 9 6 6}$ & $\mathbf{0 . 0 9 4 7}$ \\
& $(0.0000)$ & $(0.0000)$ & $(0.0000)$ & $(0.0004)$ \\
& & & & \\
Coefficient & $\mathbf{- 0 . 1 3 9 8}$ & $\mathbf{- 0 . 1 8 1 3}$ & $\mathbf{- 0 . 2 3 6 5}$ & $\mathbf{- 0 . 2 7 6 8}$ \\
& $(0.0001)$ & $(0.0000)$ & $(0.0000)$ & $(0.0005)$ \\
$R_{a d j}^{2}$ & 0.0565 & 0.0521 & 0.0657 & 0.0441 \\
\hline
\end{tabular}

Panel C: 12-Month Market Returns

\begin{tabular}{lcccc}
\hline & $B D_{E W}^{3 M}$ & $B D_{E W}^{6 M}$ & $B D_{E W}^{12 M}$ & $B D_{E W}^{36 M}$ \\
\hline Constant & $\mathbf{0 . 1 4 8 6}$ & $\mathbf{0 . 1 9 5 1}$ & $\mathbf{0 . 1 8 7 0}$ & $\mathbf{0 . 1 6 3 8}$ \\
& $(0.0057)$ & $(0.0003)$ & $(0.0004)$ & $(0.0034)$ \\
& & & & \\
Coefficient & $\mathbf{- 0 . 1 9 9 1}$ & $\mathbf{- 0 . 4 1 4 7}$ & $\mathbf{- 0 . 4 6 9 9}$ & $\mathbf{- 0 . 4 4 4 9}$ \\
& $(0.0991)$ & $(0.0071)$ & $(0.0112)$ & $(0.0530)$ \\
$R_{a d j}^{2}$ & 0.0360 & 0.0933 & 0.0773 & 0.0328 \\
\hline
\end{tabular}

Note: Table 4.3 shows the results of the predictive regressions comparable to Table 4.2 , with non-overlapping periods of the dependent variable. The results displayed in the table above used the $1^{\text {st }}$ observation in April 1964 and every $4^{t h}\left(7^{t h}, 13^{t h}\right)$ observation, depending on the length of the overlapping in the market return. The results are robust to different starting points and the results are representative for all variations. The adjusted $R_{a d j}^{2}$ of the predictive regressions are given in the last row of the table. The calculations use the Newey-West estimator with corresponding lags to account for heteroscedasticity and autocorrelation in the residuals. The p-value is given in parenthesis for every coefficient. Coefficients that are significant at least at a $10 \%$ level are printed in boldface. 
Table 4.3 displays results that are exemplary for regressions starting in April 1964. The qualitative results and interpretation of the non-overlapping regression outcome are the same as those of the regression with correction. ${ }^{5}$ This analysis confirms that the predictability of the beta dispersion is not due to statistical artifacts.

The rationale of the beta dispersion is based on the ability of the beta dispersion to predict the magnitude of a market downturn, but not be the trigger. A dummy will be included in the regression capturing whether the market has been already hit by a systematic shock, observable through negative market returns in the month prior to the estimation of the beta dispersion. This can be done by the following regression:

$$
R_{M, t}=\alpha+\beta_{1} \cdot D_{t-2} \cdot B D_{t-1}+\beta_{2} \cdot B D_{t-1}+\varepsilon_{t}
$$

where all variables correspond to Equation 4.7 extended by the dummy $D_{t-2}$ times the beta dispersion. The dummy becomes one if the market return in $t-2$ is negative and becomes zero if it is positive. This explicitly measures the predictability of the beta dispersion after the market experiences a systematic shock and specifically quantifies the described cascading effect. Table 4.4 shows the results of these regressions.

\footnotetext{
${ }^{5}$ For reasons of clarity, only the regressions of the deconstructed time series starting in April 1964 are presented in the study. All other starting points lead to comparable results and are available upon request.
} 
Table 4.4: Predictive Regressions with Dummy for Negative Market Returns

Panel A: 1-Month Market Returns

\begin{tabular}{lrrrr}
\hline & $B D_{E W}^{3 M}$ & $B D_{E W}^{6 M}$ & \multicolumn{1}{c}{$B D_{E W}^{12 M}$} & \multicolumn{1}{c}{$B D_{E W}^{36 M}$} \\
\hline$D_{t-2} \cdot B D_{t-1}$ & $\mathbf{- 0 . 0 1 0 1}$ & $\mathbf{- 0 . 0 1 3 4}$ & $\mathbf{- 0 . 0 1 2 0}$ & -0.0117 \\
& $(0.0494)$ & $(0.0569)$ & $(0.0978)$ & $(0.1353)$ \\
$B D_{t-1}$ & & & & \\
& $\mathbf{- 0 . 0 1 7 4}$ & $\mathbf{- 0 . 0 2 4 6}$ & $\mathbf{- 0 . 0 3 3 7}$ & $\mathbf{- 0 . 0 3 9 7}$ \\
& $(0.0510)$ & $(0.0470)$ & $(0.0241)$ & $(0.0473)$ \\
$R_{a d j}^{2}$ & 0.0187 & 0.0103 & 0.0115 & 0.0075 \\
\hline
\end{tabular}

Panel B: 3-Month Market Returns

\begin{tabular}{lrrrr}
\hline & $B D_{E W}^{3 M}$ & $B D_{E W}^{6 M}$ & \multicolumn{1}{c}{$B D_{E W}^{12 M}$} & \multicolumn{1}{c}{$B D_{E W}^{36 M}$} \\
\hline$D_{t-2} \cdot B D_{t-1}$ & $\mathbf{- 0 . 0 2 7 8}$ & $\mathbf{- 0 . 0 3 2 7}$ & -0.0273 & -0.0257 \\
& $(0.0319)$ & $(0.0524)$ & $(0.1016)$ & $(0.1376)$ \\
$B D_{t-1}$ & & & & \\
& $\mathbf{- 0 . 0 7 3 6}$ & $\mathbf{- 0 . 1 0 9 4}$ & $\mathbf{- 0 . 1 3 2 4}$ & $\mathbf{- 0 . 1 6 7 9}$ \\
$R_{a d j}^{2}$ & $(0.0057)$ & $(0.0180)$ & $(0.0182)$ & $(0.0250)$ \\
\hline
\end{tabular}

Panel C: 6-Month Market Returns

\begin{tabular}{lrrrr}
\hline & $B D_{E W}^{3 M}$ & $B D_{E W}^{6 M}$ & $B D_{E W}^{12 M}$ & \multicolumn{1}{c}{$B D_{E W}^{36 M}$} \\
\hline$D_{t-2} \cdot B D_{t-1}$ & $\mathbf{- 0 . 0 4 7 0}$ & $\mathbf{- 0 . 0 5 1 3}$ & $\mathbf{- 0 . 0 4 5 0}$ & -0.0441 \\
& $(0.0177)$ & $(0.0377)$ & $(0.0748)$ & $(0.1131)$ \\
& & & & \\
$B D_{t-1}$ & $\mathbf{0 . 1 3 0 0}$ & $\mathbf{- 0 . 2 0 2 9}$ & $\mathbf{- 0 . 2 2 8 2}$ & $\mathbf{- 0 . 2 7 4 8}$ \\
& $(0.0014)$ & $(0.0024)$ & $(0.0098)$ & $(0.0380)$ \\
$R_{a d j}^{2}$ & 0.0270 & 0.0272 & 0.0237 & 0.0200 \\
\hline
\end{tabular}

Panel D: 12-Month Market Returns

\begin{tabular}{lrrrr}
\hline & $B D_{E W}^{3 M}$ & $B D_{E W}^{6 M}$ & $B D_{E W}^{12 M}$ & \multicolumn{1}{c}{$B D_{E W}^{36 M}$} \\
\hline$D_{t-2} \cdot B D_{t-1}$ & $\mathbf{- 0 . 0 6 2 6}$ & $\mathbf{- 0 . 0 6 6 6}$ & -0.0594 & -0.0536 \\
& $(0.0415)$ & $(0.0668)$ & $(0.1057)$ & $(0.1494)$ \\
$B D_{t-1}$ & & & & \\
& $\mathbf{- 0 . 1 9 8 9}$ & $\mathbf{- 0 . 3 0 8 1}$ & $\mathbf{- 0 . 3 5 9 0}$ & $\mathbf{- 0 . 4 0 5 0}$ \\
$R_{a d j}^{2}$ & $(0.0074)$ & $(0.0083)$ & $(0.0160)$ & $(0.0847)$ \\
\hline
\end{tabular}

Note: Table 4.4 shows the results of the predictive regressions comparable to Table 4.2, with an additional dummy $D_{t-2}$ controlling for the market return of the period prior to the estimation of the beta dispersion. The dummy variable should indicate a systematic shock in the prior period and focus on the ability of the beta dispersion to predict how pronounced will be the evolution of a market downturn, given a high beta dispersion. The adjusted $R_{a d j}^{2}$ of the predictive regressions are given in the last row of the table and the p-value is given in parenthesis for every variable. The calculations use the Britten-Jones et al. (2011) correction for overlapping periods in the dependent variable and use the Newey-West estimator with corresponding lags to account for heteroscedasticity and autocorrelation in the residuals. Coefficients that are significant at least at a $10 \%$ level are printed in boldface. 
This regression shows that the product of the dummy and the beta dispersion is meaningful. The sign of the regression coefficient of this variable is in all cases negative and mostly significant. This means that, especially, the beta dispersion in combination with a market decline can help to evaluate how hard a market will be hit by a shock. The results show that the shorter the estimation period of the betas, the more informative would be the beta dispersion. Nevertheless, the actual level of the beta dispersion still plays a role and its coefficients are still significantly negative.

As beta dispersion, on a stand-alone basis, is a promising predictor of the market return, it is tested whether the measure maintains its explanatory power if further variables that have been found to be successful predictors in previous studies are added to the Equation 4.7. Particularly, the dividend yield of the S\&P 500 Index and the short rate are used as these variables jointly predict market returns according to Ang and Bekaert (2007). Moreover, the cay factor from Lettau and Ludvigson (2001) and the average variance and average correlation (in the estimation period) of all constituents of the market from Pollet and Wilson (2010) are used. In addition, one of the most successful technical indicators according to Neely et al. (2014), the moving average of the market return as the difference between the 2-month and 12-month moving average, is included. Whaley (2009) shows the predictive characteristics of the VIX in terms of a fear index for market developments; thus, this index is also included. Finally, the return dispersion (Maio 2016) is included to control for redundant information in the two dispersion measures. Table 4.5 shows the results of the multivariate predictive regressions. 
Table 4.5: Predictive Regressions with Additional Explanatory Variables

Panel A: 1-Month Market Returns

\begin{tabular}{|c|c|c|c|c|}
\hline & $B D_{E W}^{3 M}$ & $B D_{E W}^{6 M}$ & $B D_{E W}^{12 M}$ & $B D_{E W}^{36 M}$ \\
\hline Constant & $\begin{array}{c}0.0116 \\
(0.6853)\end{array}$ & $\begin{array}{c}0.0091 \\
(0.7490)\end{array}$ & $\begin{array}{c}0.0200 \\
(0.4642)\end{array}$ & $\begin{array}{c}0.0242 \\
(0.3310)\end{array}$ \\
\hline Beta Dispersion & $\begin{array}{r}-0.0076 \\
(0.6706)\end{array}$ & $\begin{array}{c}-0.0048 \\
(0.8110)\end{array}$ & $\begin{array}{r}-0.0287 \\
(0.1021)\end{array}$ & $\begin{array}{r}-0.0429 \\
(0.2297)\end{array}$ \\
\hline Dividend Yield & $\begin{array}{c}0.0019 \\
(0.7611)\end{array}$ & $\begin{array}{c}0.0020 \\
(0.7589)\end{array}$ & $\begin{array}{c}0.0000 \\
(0.9965)\end{array}$ & $\begin{array}{r}-0.0012 \\
(0.8515)\end{array}$ \\
\hline Short Rate & $\begin{array}{c}0.0221 \\
(0.3333)\end{array}$ & $\begin{array}{c}0.0230 \\
(0.3094)\end{array}$ & $\begin{array}{c}0.0168 \\
(0.4765)\end{array}$ & $\begin{array}{c}0.0106 \\
(0.6765)\end{array}$ \\
\hline Cay Factor & $\begin{array}{r}-\mathbf{0 . 6 3 9 3} \\
(0.0095)\end{array}$ & $\begin{array}{r}-\mathbf{0 . 6 5 0 7} \\
(0.0077)\end{array}$ & $\begin{array}{r}-\mathbf{0 . 6 2 4 0} \\
(0.0069)\end{array}$ & $\begin{array}{r}-\mathbf{0 . 5 9 7 3} \\
(0.0153)\end{array}$ \\
\hline Aver. Variance & $\begin{array}{r}-\mathbf{0 . 2 5 8 3} \\
(0.0225)\end{array}$ & $\begin{array}{r}-\mathbf{0 . 2 7 2 0} \\
(0.0094)\end{array}$ & $\begin{array}{r}-\mathbf{0 . 2 5 2 1} \\
(0.0275)\end{array}$ & $\begin{array}{r}-\mathbf{0 . 2 6 7 9} \\
(0.0081)\end{array}$ \\
\hline Aver. Correlation & $\begin{array}{c}0.0321 \\
(0.5924)\end{array}$ & $\begin{array}{c}0.0385 \\
(0.5012)\end{array}$ & $\begin{array}{c}0.0222 \\
(0.7128)\end{array}$ & $\begin{array}{c}0.0384 \\
(0.5065)\end{array}$ \\
\hline $\operatorname{MA}(2,12)$ & $\begin{array}{r}-\mathbf{0 . 0 0 1 5} \\
(0.0111)\end{array}$ & $\begin{array}{r}-\mathbf{0 . 0 0 1 5} \\
(0.0106)\end{array}$ & $\begin{array}{r}-\mathbf{0 . 0 0 1 5} \\
(0.0078)\end{array}$ & $\begin{array}{r}-\mathbf{0 . 0 0 1 6} \\
(0.0046)\end{array}$ \\
\hline VIX & $\begin{array}{l}\mathbf{0 . 0 0 1 6} \\
(0.0159)\end{array}$ & $\begin{array}{l}\mathbf{0 . 0 0 1 6} \\
(0.0104)\end{array}$ & $\begin{array}{l}\mathbf{0 . 0 0 1 6} \\
(0.0140)\end{array}$ & $\begin{array}{c}\mathbf{0 . 0 0 1 6} \\
(0.0087)\end{array}$ \\
\hline Return Dispersion & $\begin{array}{c}-0.7180 \\
(0.1157)\end{array}$ & $\begin{array}{c}-0.7228 \\
(0.1135)\end{array}$ & $\begin{array}{c}-0.6909 \\
(0.1492)\end{array}$ & $\begin{array}{r}-0.6296 \\
(0.2145)\end{array}$ \\
\hline$R_{a d j}^{2}$ & 0.0568 & 0.0563 & 0.0619 & 0.0611 \\
\hline
\end{tabular}


Panel B: 3-Month Market Returns

\begin{tabular}{|c|c|c|c|c|}
\hline & $B D_{E W}^{3 M}$ & $B D_{E W}^{6 M}$ & $B D_{E W}^{12 M}$ & $B D_{E W}^{36 M}$ \\
\hline Constant & $\begin{array}{c}0.0455 \\
(0.6468)\end{array}$ & $\begin{array}{c}0.0516 \\
(0.5987)\end{array}$ & $\begin{array}{c}0.0810 \\
(0.3898)\end{array}$ & $\begin{array}{c}0.1326 \\
(0.1318)\end{array}$ \\
\hline Beta Dispersion & $\begin{array}{r}-0.0410 \\
(0.3985)\end{array}$ & $\begin{array}{c}-0.0673 \\
(0.2613)\end{array}$ & $\begin{array}{r}-\mathbf{0 . 1 3 1 9} \\
(0.0235)\end{array}$ & $\begin{array}{r}-\mathbf{0 . 2 7 8 7} \\
(0.0120)\end{array}$ \\
\hline Dividend Yield & $\begin{array}{c}0.0070 \\
(0.7728)\end{array}$ & $\begin{array}{c}0.0049 \\
(0.8418)\end{array}$ & $\begin{array}{c}-0.0017 \\
(0.9444)\end{array}$ & $\begin{array}{c}-0.0135 \\
(0.5540)\end{array}$ \\
\hline Short Rate & $\begin{array}{c}0.0587 \\
(0.5590)\end{array}$ & $\begin{array}{c}0.0541 \\
(0.5883)\end{array}$ & $\begin{array}{c}0.0355 \\
(0.7225)\end{array}$ & $\begin{array}{c}-0.0193 \\
(0.8532)\end{array}$ \\
\hline Cay Factor & $\begin{array}{c}-0.8787 \\
(0.3380)\end{array}$ & $\begin{array}{c}-0.8652 \\
(0.3486)\end{array}$ & $\begin{array}{c}-0.8227 \\
(0.3778)\end{array}$ & $\begin{array}{c}-0.5878 \\
(0.5636)\end{array}$ \\
\hline Aver. Variance & $\begin{array}{c}-0.4486 \\
(0.3424)\end{array}$ & $\begin{array}{c}-0.4501 \\
(0.3472)\end{array}$ & $\begin{array}{r}-0.4384 \\
(0.3699)\end{array}$ & $\begin{array}{r}-0.4915 \\
(0.2863)\end{array}$ \\
\hline Aver. Correlation & $\begin{array}{c}0.2233 \\
(0.2087)\end{array}$ & $\begin{array}{c}0.2092 \\
(0.2317)\end{array}$ & $\begin{array}{c}0.1893 \\
(0.2671)\end{array}$ & $\begin{array}{c}0.2469 \\
(0.1402)\end{array}$ \\
\hline $\mathrm{MA}(2,12)$ & $\begin{array}{r}-0.0035 \\
(0.1401)\end{array}$ & $\begin{array}{c}-0.0035 \\
(0.1463)\end{array}$ & $\begin{array}{r}-0.0037 \\
(0.1297)\end{array}$ & $\begin{array}{r}-\mathbf{0 . 0 0 4 3} \\
(0.0810)\end{array}$ \\
\hline VIX & $\begin{array}{l}\mathbf{0 . 0 0 3 1} \\
(0.0121)\end{array}$ & $\begin{array}{l}\mathbf{0 . 0 0 3 2} \\
(0.0110)\end{array}$ & $\begin{array}{l}\mathbf{0 . 0 0 3 4} \\
(0.0079)\end{array}$ & $\begin{array}{l}\mathbf{0 . 0 0 3 3} \\
(0.0093)\end{array}$ \\
\hline Return Dispersion & $\begin{array}{r}-\mathbf{2 . 7 5 2 3} \\
(0.0035)\end{array}$ & $\begin{array}{r}-\mathbf{2 . 6 7 1 0} \\
(0.0044)\end{array}$ & $\begin{array}{r}-\mathbf{2 . 6 2 4 3} \\
(0.0051)\end{array}$ & $\begin{array}{r}\mathbf{- 2 . 1 5 5 2} \\
(0.0333)\end{array}$ \\
\hline$R_{a d j}^{2}$ & 0.0551 & 0.0557 & 0.0610 & 0.0624 \\
\hline
\end{tabular}


Panel C: 6-Month Market Returns

\begin{tabular}{|c|c|c|c|c|}
\hline & $B D_{E W}^{3 M}$ & $B D_{E W}^{6 M}$ & $B D_{E W}^{12 M}$ & $B D_{E W}^{36 M}$ \\
\hline Constant & $\begin{array}{c}0.1392 \\
(0.3856)\end{array}$ & $\begin{array}{c}0.1612 \\
(0.3205)\end{array}$ & $\begin{array}{c}0.1855 \\
(0.2540)\end{array}$ & $\begin{array}{l}\mathbf{0 . 2 6 9 9} \\
(0.0665)\end{array}$ \\
\hline Beta Dispersion & $\begin{array}{r}-\mathbf{0 . 1 5 7 4} \\
(0.0171)\end{array}$ & $\begin{array}{r}-\mathbf{0 . 2 5 1 0} \\
(0.0077)\end{array}$ & $\begin{array}{r}-\mathbf{0 . 3 0 5 7} \\
(0.0031)\end{array}$ & $\begin{array}{r}-\mathbf{0 . 5 6 6 3} \\
(0.0020)\end{array}$ \\
\hline Dividend Yield & $\begin{array}{c}0.0113 \\
(0.7487)\end{array}$ & $\begin{array}{c}0.0031 \\
(0.9321)\end{array}$ & $\begin{array}{c}-0.0067 \\
(0.8603)\end{array}$ & $\begin{array}{c}-0.0267 \\
(0.4522)\end{array}$ \\
\hline Short Rate & $\begin{array}{c}-0.0176 \\
(0.9138)\end{array}$ & $\begin{array}{c}-0.0338 \\
(0.8361)\end{array}$ & $\begin{array}{c}-0.0542 \\
(0.7448)\end{array}$ & $\begin{array}{c}-0.1548 \\
(0.3525)\end{array}$ \\
\hline Cay Factor & $\begin{array}{c}-0.5110 \\
(0.7367)\end{array}$ & $\begin{array}{c}-0.4660 \\
(0.7600)\end{array}$ & $\begin{array}{c}-0.5328 \\
(0.7294)\end{array}$ & $\begin{array}{c}-0.1103 \\
(0.9487)\end{array}$ \\
\hline Aver. Variance & $\begin{array}{c}-0.1219 \\
(0.8118)\end{array}$ & $\begin{array}{c}-0.1387 \\
(0.7858)\end{array}$ & $\begin{array}{r}-0.2685 \\
(0.6329)\end{array}$ & $\begin{array}{r}-0.4138 \\
(0.4578)\end{array}$ \\
\hline Aver. Correlation & $\begin{array}{c}0.1199 \\
(0.6535)\end{array}$ & $\begin{array}{c}0.0804 \\
(0.7591)\end{array}$ & $\begin{array}{c}0.1501 \\
(0.5369)\end{array}$ & $\begin{array}{c}0.2901 \\
(0.2189)\end{array}$ \\
\hline $\mathrm{MA}(2,12)$ & $\begin{array}{c}-0.0050 \\
(0.2218)\end{array}$ & $\begin{array}{c}-0.0049 \\
(0.2293)\end{array}$ & $\begin{array}{r}-0.0057 \\
(0.1749)\end{array}$ & $\begin{array}{r}-0.0066 \\
(0.1158)\end{array}$ \\
\hline VIX & $\begin{array}{l}\mathbf{0 . 0 0 3 4} \\
(0.0487)\end{array}$ & $\begin{array}{l}\mathbf{0 . 0 0 4 0} \\
(0.0263)\end{array}$ & $\begin{array}{l}\mathbf{0 . 0 0 4 5} \\
(0.0147)\end{array}$ & $\begin{array}{l}\mathbf{0 . 0 0 4 3} \\
(0.0187)\end{array}$ \\
\hline Return Dispersion & $\begin{array}{r}-\mathbf{3 . 4 9 3 6} \\
(0.0067)\end{array}$ & $\begin{array}{r}-\mathbf{3 . 2 1 7 3} \\
(0.0099)\end{array}$ & $\begin{array}{r}-\mathbf{3 . 3 7 6 7} \\
(0.0083)\end{array}$ & $\begin{array}{r}-\mathbf{2 . 4 7 1 1} \\
(0.0955)\end{array}$ \\
\hline$R_{a d j}^{2}$ & 0.0477 & 0.0505 & 0.0518 & 0.0502 \\
\hline
\end{tabular}


Panel D: 12-Month Market Returns

\begin{tabular}{|c|c|c|c|c|}
\hline & $B D_{E W}^{3 M}$ & $B D_{E W}^{6 M}$ & $B D_{E W}^{12 M}$ & $B D_{E W}^{3 M}$ \\
\hline Constant & $\begin{array}{c}0.2691 \\
(0.2089)\end{array}$ & $\begin{array}{c}0.2912 \\
(0.1852)\end{array}$ & $\begin{array}{c}0.3186 \\
(0.1263)\end{array}$ & $\begin{array}{c}0.3980 \\
(0.0442)\end{array}$ \\
\hline Beta Dispersion & $\begin{array}{r}-\mathbf{0 . 2 7 9 8} \\
(0.0012)\end{array}$ & $\begin{array}{r}-\mathbf{0 . 4 0 9 6} \\
(0.0013)\end{array}$ & $\begin{array}{r}-\mathbf{0 . 4 8 4 0} \\
(0.0021)\end{array}$ & $\begin{array}{r}-\mathbf{0 . 7 7 1 4} \\
(0.0358)\end{array}$ \\
\hline Dividend Yield & $\begin{array}{c}0.0396 \\
(0.3118)\end{array}$ & $\begin{array}{c}0.0270 \\
(0.5153)\end{array}$ & $\begin{array}{c}0.0139 \\
(0.7383)\end{array}$ & $\begin{array}{r}-0.0068 \\
(0.8637)\end{array}$ \\
\hline Short Rate & $\begin{array}{c}-0.2268 \\
(0.3612)\end{array}$ & $\begin{array}{c}-0.2461 \\
(0.3277)\end{array}$ & $\begin{array}{r}-0.2747 \\
(0.2672)\end{array}$ & $\begin{array}{r}-\mathbf{0 . 3 9 3 1} \\
(0.0987)\end{array}$ \\
\hline Cay Factor & $\begin{array}{c}2.0012 \\
(0.3216)\end{array}$ & $\begin{array}{c}2.0103 \\
(0.3343)\end{array}$ & $\begin{array}{c}1.8607 \\
(0.3905)\end{array}$ & $\begin{array}{c}2.3436 \\
(0.3189)\end{array}$ \\
\hline Aver. Variance & $\begin{array}{c}0.1560 \\
(0.7781)\end{array}$ & $\begin{array}{c}0.0663 \\
(0.9031)\end{array}$ & $\begin{array}{r}-0.1606 \\
(0.7759)\end{array}$ & $\begin{array}{r}-0.4275 \\
(0.4872)\end{array}$ \\
\hline Aver. Correlation & $\begin{array}{r}-0.1643 \\
(0.7416)\end{array}$ & $\begin{array}{c}-0.1836 \\
(0.7049)\end{array}$ & $\begin{array}{r}-0.0657 \\
(0.8867)\end{array}$ & $\begin{array}{c}0.1746 \\
(0.7013)\end{array}$ \\
\hline $\mathrm{MA}(2,12)$ & $\begin{array}{r}-0.0052 \\
(0.4018)\end{array}$ & $\begin{array}{c}-0.0052 \\
(0.4117)\end{array}$ & $\begin{array}{r}-0.0062 \\
(0.3336)\end{array}$ & $\begin{array}{r}-0.0074 \\
(0.2424)\end{array}$ \\
\hline VIX & $\begin{array}{c}0.0030 \\
(0.2043)\end{array}$ & $\begin{array}{c}0.0040 \\
(0.1085)\end{array}$ & $\begin{array}{l}\mathbf{0 . 0 0 4 8} \\
(0.0588)\end{array}$ & $\begin{array}{l}\mathbf{0 . 0 0 4 4} \\
(0.0925)\end{array}$ \\
\hline Return Dispersion & $\begin{array}{r}-\mathbf{5 . 4 2 1 1} \\
(0.0070)\end{array}$ & $\begin{array}{r}-4.9928 \\
(0.0155)\end{array}$ & $\begin{array}{r}-\mathbf{5 . 1 8 0 9} \\
\quad(0.0182)\end{array}$ & $\begin{array}{r}-3.9440 \\
(0.1126)\end{array}$ \\
\hline$R_{a d j}^{2}$ & 0.0526 & 0.0527 & 0.0501 & 0.0445 \\
\hline
\end{tabular}

Note: Table 4.5 shows the results of the predictive regressions with nine independent variables and 1-, 3-, 6-, and 12-month log excess return of the S\&P 500 Index as dependent variables (Panel A, Panel B, Panel C, and Panel D). The regression equation has the following independent variables: beta dispersion, dividend yield, short rate, cay factor, average variance, average correlation, moving average, VIX, and return dispersion. Dividend yield, average variance, average correlation, moving average, and return dispersion refer to the S\&P 500 Index, and short rate is the 1-month T-bill rate. Additionally, the cay factor is provided by Lettau's database. The beta dispersion is calculated based on 3, 6, 12, and 36 months. The in-sample, adjusted $R_{a d j}^{2}$, of the predictive regressions is given in the last row of the table. Overlapping periods of the dependent variable are addressed by using the correction proposed by Britten-Jones et al. (2011). The calculations of the significance levels use the Newey-West estimator with corresponding lags to account for heteroscedasticity and autocorrelation in the residuals and the p-value is given in parenthesis for every coefficient. Coefficients that are significant at least at a $10 \%$ level are printed in boldface. 
All coefficients of the beta dispersion measure retain their signs. For the 1-month prediction (Panel A), the beta dispersion does not contribute toward prediction accuracy. The major contributors toward this prediction are the cay factor, average variance, moving average, and VIX. For the 3-month market return (Panel B), the beta dispersion adds value, especially when it is estimated over a longer horizon. Concerning prediction for the 6-month and 12-month market returns (Panel C and D), beta dispersion is considered one of the most valuable contributors to the prediction accuracy, besides the VIX and the return dispersion. The multivariate prediction has a much higher predictive accuracy than the simple regression, and the $R_{a d j}^{2}$ are comparable to prior research. Nevertheless, the beta dispersion captures specific information that cannot be covered by other predictors.

\subsubsection{Predicting Market Returns - Out-of-sample Evaluation}

The in-sample evaluation of beta dispersion suggests that its level can be used, to some extent, to predict future market returns and predict the extent of the market downturn. The economic concept would gain much more strength if the results also hold in the out-of-sample evaluation. Two commonly used out-of-sample approaches are performed. The out-of-sample $R_{O S}^{2}$, on the one hand, aggregates the evaluation in one figure and the cumulative sum of differences in the squared forecast errors (CSDFE), on the other hand, evaluates the superior prediction out-of-sample graphically. The calculation of the out-of-sample $R_{O S}^{2}$ follows an established approach (Campbell and Thompson 2008; Neely et al. 2014). This measure compares the forecast of a predictor to the forecast of a benchmark, typically the historical market return, which is commonly seen as the most stringent benchmark for equity premium predictors (Goyal and Welch 2003; Welch and Goyal 2008). The out-of-sample $R_{O S}^{2}$ is calculated as shown in Equation 4.9:

$$
R_{O S}^{2}=1-\frac{\sum_{t=1}^{T}\left(R_{M, t}-\hat{R}_{M, t}\right)^{2}}{\sum_{t=1}^{T}\left(R_{M, t}-\bar{R}_{M, t}\right)^{2}},
$$

where $R_{M, t}$ is the actual observed market return in $t, \hat{R}_{M, t}$ is the predicted market return by the BD measure, and $\bar{R}_{M, t}$ is the benchmark prediction. In this study, initially, the 
historical mean market return is estimated prior to the actual observed market return and presents the benchmark prediction. The value of the $R_{O S}^{2}$ ranges between $(-\infty, 1]$. The data sample is divided into two subsamples to estimate predictive regressions in the first and calculate the return predictions and the $R_{O S}^{2}$ in the second. The choice of the split point is based on the argumentation in Neely et al. (2014). The first subsample should be long enough to estimate stable regression coefficients, but at the same time the second subsample (i.e., the evaluation sample) should be large enough to have sufficient data for the evaluation. This is because the power of the forecast tests increases with this size (also see Hansen and Timmermann (2012)). A positive out-of-sample $R_{O S}^{2}$ indicates a superior forecasting performance of the tested measure against the benchmark. I use the first 20 years as the first subsample to determine the initial regression coefficients and extend this window monthly. At the end of every month, a predictive regression with all available information at that point is performed to estimate the regression coefficients. Together with the currently observed beta dispersion, the forecast of the market return is calculated (also see Campbell and Thompson (2008)). This forecast is compared to the prediction of the benchmark $\bar{R}_{t}$, calculated over the same period as the regression coefficients, with Equation 4.9. More recent research argues that the historical mean of the market risk premium is not the most appropriate benchmark (Constantinides 2002; Ibbotson and Chen 2003; Donaldson et al. 2010). Therefore, three further benchmarks suggested in current literature are included. These benchmarks are the market risk premium calculated with the CRSP (Center for Research in Security Prices) value-weighted index, a fixed market risk premium of $5.1 \%$ (both in Avdis and Wachter (2017)), and SVIX measure proposed by Martin (2017). The results of the $R_{O S}^{2}$ calculation are shown in Table 4.6. 
Table 4.6: Results of the Out-of-sample $R_{O S}^{2}$ for $B D_{E W}$

Panel A: 20-year Initial Estimation, Historical Mean of S\&P 500

\begin{tabular}{lllll}
\hline & $B D_{E W}^{3 M}$ & \multicolumn{1}{c}{$B D_{E W}^{6 M}$} & $B D_{E W}^{12 M}$ & $B D_{E W}^{36 M}$ \\
\hline 1 Month & 0.0040 & 0.0041 & 0.0078 & $0.0031^{*}$ \\
3 Months & 0.0003 & $0.0074^{*}$ & $0.0178^{* *}$ & $0.0110^{* *}$ \\
6 Months & $0.0133^{* *}$ & $0.0301^{* * *}$ & $0.0425^{* * *}$ & $0.0244^{* *}$ \\
12 Months & $0.0215^{* * *}$ & $0.0515^{* * *}$ & $0.0545^{* * *}$ & $0.0104^{* * *}$ \\
\hline
\end{tabular}

Panel B: 20-year Initial Estimation, Historical Mean of CRSP index

\begin{tabular}{lllll}
\hline & $B D_{E W}^{3 M}$ & \multicolumn{1}{c}{$B D_{E W}^{6 M}$} & \multicolumn{1}{c}{$B D_{E W}^{12 M}$} & \multicolumn{1}{c}{$B D_{E W}^{36 M}$} \\
\hline 1 Month & 0.0090 & 0.0091 & $0.0128^{*}$ & 0.0081 \\
3 Months & 0.0047 & $0.0118^{*}$ & $0.0222^{* *}$ & $0.0153^{*}$ \\
$\approx$ & $0.0151^{* *}$ & $0.0318^{* * *}$ & $0.0442^{* * *}$ & $0.0261^{* *}$ \\
12 Months & $0.0172^{* *}$ & $0.0473^{* *}$ & $0.0503^{* * *}$ & $0.0060^{* *}$ \\
\hline
\end{tabular}

Panel C: 20-year Initial Estimation, Fix Market Risk Premium of 5.1\%

\begin{tabular}{lllll}
\hline & $B D_{E W}^{3 M}$ & $B D_{E W}^{6 M}$ & $B D_{E W}^{12 M}$ & $B D_{E W}^{36 M}$ \\
\hline 1 Month & $0.0080^{*}$ & 0.0082 & $0.0118^{*}$ & 0.0071 \\
\multirow{2}{*}{ 3 Months } & $0.0099^{* *}$ & $0.0170^{* *}$ & $0.0273^{* * *}$ & $0.0205^{* *}$ \\
6 Months & $0.0270^{* * *}$ & $0.0436^{* * *}$ & $0.0558^{* * *}$ & $0.0380^{* * *}$ \\
12 Months & $0.0387^{* * *}$ & $0.0682^{* * *}$ & $0.0711^{* * *}$ & $0.0278^{* * *}$ \\
\hline
\end{tabular}

Panel D: 20-year Initial Estimation, $S V I X^{2}$ Following (Martin(2016))

\begin{tabular}{|c|c|c|c|c|c|}
\hline & & $B D_{E W}^{3 M}$ & $B D_{E W}^{6 M}$ & $B D_{E W}^{12 M}$ & $B D_{E W}^{36 M}$ \\
\hline$R_{M}$ & 1 Month & $0.0390^{* *}$ & $0.0400 * * *$ & $0.0400^{* * *}$ & $0.0390 * * *$ \\
\hline
\end{tabular}

Note: Table 4.6 shows the out-of-sample $R_{O S}^{2}$ for the prediction of the beta dispersion in comparison to the benchmark prediction for 1-, 3-, 6-, and 12-month log excess market return $\left(R_{M, t}\right)$. The out-of-sample $R_{O S}^{2}$ is calculated via Equation 4.9. The forecasts are estimated by using the predictive regression coefficients from a dynamically enlarged time series of beta dispersion and market return, which includes the whole period prior to the currently observed dispersion. The forecasts, taken from the monthly adjusted regression, are set in relation to different benchmark forecasts of the market risk premium. These benchmarks are historical mean of the S\&P 500 excess returns, the historical mean of the CRSP value-weighted index, a commonly used constant of $5.1 \%$, and a VIX measure proposed by Martin (2017). As the VIX is based on options with a maturity of one month, only the 1-month market return can be predicted. A positive out-of-sample $R_{O S}^{2}$ indicates a superior forecasting performance of the beta dispersion. The significance level of the $R_{O S}^{2}$ is estimated based on the Clark and West (2007) MSFE-adjusted statistic for testing the null hypothesis that the benchmark forecast estimation error is less than or equal to the beta dispersion forecast error. Significance level: *** $0.01, * * 0.05$, and $* 0.1$. 
The $R_{O S}^{2}$ confirms the predictive dominance of the beta dispersion (3-, 6-, and 12-month estimation) for mid- and long-term predictions (6 and 12 months). This is represented by a positive $R_{O S}^{2}$. This out-of-sample predictability applies for all different benchmarks. This is remarkable because other predictors often lack out-of-sample predictability. The significance test of $R_{O S}^{2}$ is estimated based on Clark and West (2007)'s MSFE-adjusted (mean square forecast error) statistic for testing the null hypothesis that the benchmark forecast estimation error is less than or equal to the competing forecast error. Convincingly, the $R_{O S}^{2}$ are not only positive but also significant. This affirms the conclusion that the beta dispersion is a reliable predictor of the market return, even in an out-of-sample framework. A second way of analyzing the out-of-sample predictability is to examine the superior performance over time. The cumulative sum of differences in the squared forecast errors (CSDFE) is a useful tool for this investigation (Goyal and Welch 2003; Welch and Goyal 2008). It reminds of the $R_{O S}^{2}$, but it results in a time series that can be graphically studied and is calculated as presented in the Equation 4.10:

$$
\mathrm{CSDFE}_{T}=\sum_{t=1}^{T}\left(\left(R_{M, t}-\bar{R}_{M, t}\right)^{2}-\left(R_{M, t}-\hat{R}_{M, t}\right)^{2}\right)
$$

It shows the dynamics of the predictive performance over time and helps to distinguish between periods where the beta dispersion is a more accurate predictor compared to the benchmark prediction. If the $\mathrm{CSDFE}_{T}$ is positive, then the beta dispersion would be considered a superior predictor compared to the benchmark and vice versa. In Figure 4.3, the time series of CSDFE for the 6-month market return prediction is displayed. ${ }^{6}$

\footnotetext{
${ }^{6}$ The time series of CSDFE based on other specifications of the beta dispersion do not vary noticeably from Figure 4.3.
} 


\section{Figure 4.3: Cumulative Sum of Differences in the Squared Forecast Errors}

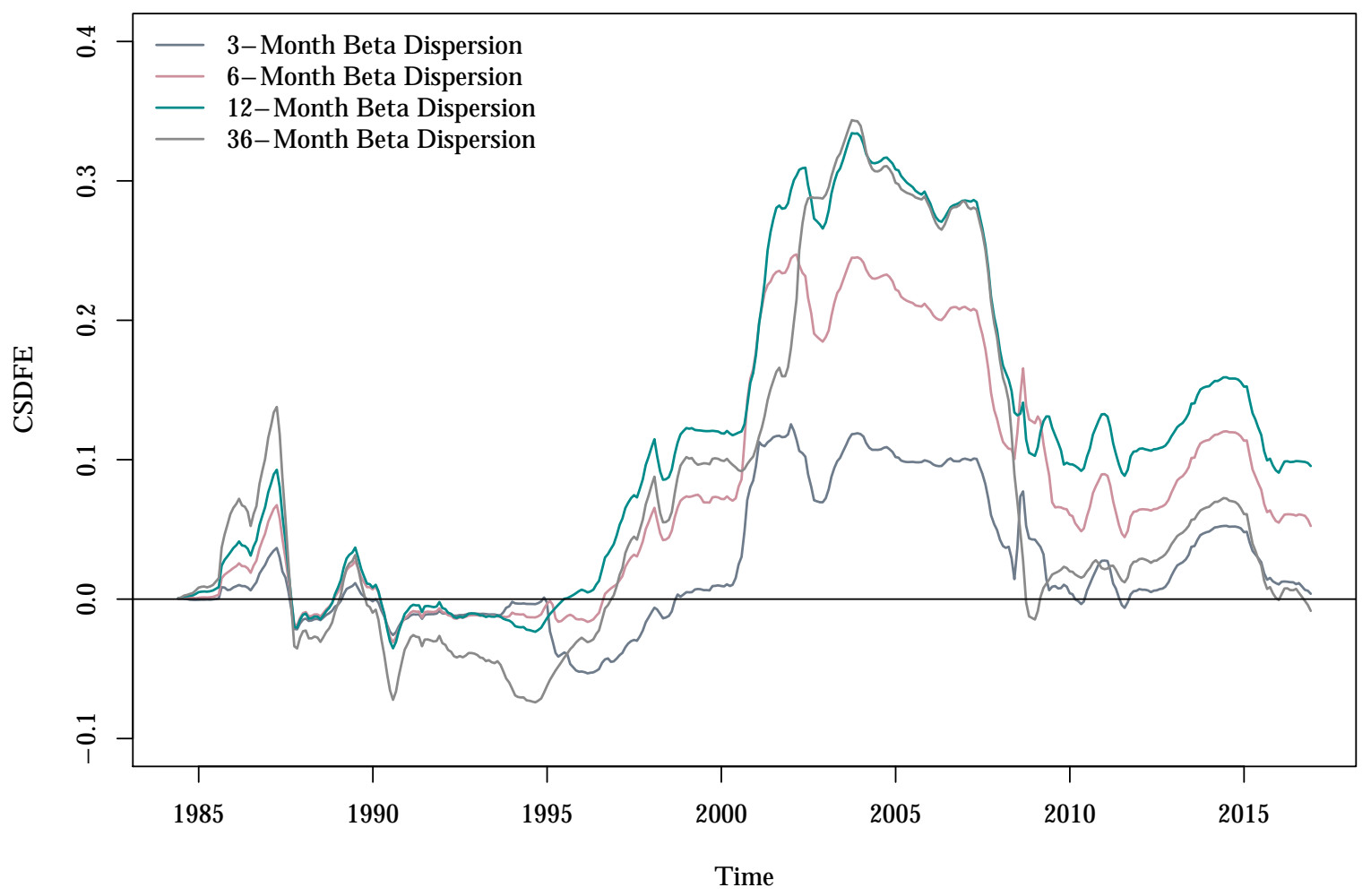

Note: Figure 4.3 shows the time series of the CSDFE, following Goyal and Welch (2003) and Welch and Goyal (2008), over the period April 1984 to December 2016. This period is shorter than the total sampled period because the first 20 years of the sample are used to estimate the initial regression coefficients. It shows the dynamic predictive performance over time and helps to distinguish between periods where the beta dispersion is a superior predictor of future market return when compared to the historical market risk premium and where the beta dispersion is an inferior predictor. If the CSDFE are positive, it would indicate a superior performance of the beta dispersion when compared to the historical market risk premium.

It can be seen that the beta dispersion has been effectively predicting the market return more recently, since the late 1990s. Especially, before market crises in 1987, 2001, and 2008, the beta dispersion seemed valuable. After the shocks in 1987 and 2008, the beta dispersion lost its predictive power. This might be attributed to the fact that these crises occurred at a rapid pace and incurred higher losses and the drop of the market was more extreme than during the dotcom bubble. The economic explanation of the beta dispersion is based on the cascading effect. In a disturbed market environment like this, it is doubtful whether the monthly calculated beta dispersion can capture these short-term effects correctly. 
Putting together the in-sample and out-of-sample evaluation of the beta dispersion measures, the empirical results highlight the appropriateness of the economic idea of beta dispersion. The BD measure, which takes all stocks into account, delivers slightly more reliable and stronger results than the QBD measure, which is explicitly based on the extreme tails of the beta distribution. In the initial predictive regression analyses, it is accounted for the overlapping periods in two different ways that lead to identical results. By including a dummy variable in the regression, it can be seen that the beta dispersion is especially important in helping to determine the magnitude of the downturn during market declines. The multivariate regressions show that the proposed measures contain additional information compared to already studied predictors, especially in the prediction of the medium- and long-term market return. In addition, the computed out-of-sample $R_{O S}^{2}$ shows that the dispersion measure is valuable even when comparing the beta dispersion to stringent benchmarks. The time series analysis of the predictive ability of the dispersion confirms that the beta dispersion has been apparently superior to its benchmark since the late 1990s. This enhances the results of the out-of-sample $R_{O S}^{2}$ because the worse performance seems to be caused by earlier observations from the late 1980s to the late 1990s. The predictor suggested in this study clearly complements and partially outperforms already examined predictors in the literature. This corroborates the results from the decomposition of returns and emphasizes the relevance of the beta dispersion being a measure of market vulnerability.

\subsubsection{Market Timing Strategies Using Beta Dispersion}

To evaluate the usefulness of the beta dispersion for an investor and to show further economic significance of the beta dispersion, market timing strategies based on this measure are implemented to obtain a comprehensive view. Furthermore, this section introduces distributional regressions to finance and shows their beneficial contribution toward market timing strategies. Distributional regressions comprise a new modeling approach for determining the probability distribution function of a variable, conditional on another observable variable (Silbersdorff 2017). ${ }^{7}$ The market timing strategies use the

\footnotetext{
${ }^{7}$ Further information on the estimation of distributional regressions is provided in the Appendix 4.A.3.
} 
probability distribution to trigger the investment decision. If the probability that the market return will be positive, conditional on the observed beta dispersion, is greater than $50 \%$, the strategies would drive investments in the market portfolio. Three different versions of implementing such a strategy are explored. The first version involves the usual approach and shifts wealth between money and stock market; the second version shifts wealth between a short and a long position in the market; and the third version makes this shift with a fraction of the wealth, dependent on the aforementioned probability. The possibility to be long and short in the market at considerably low transaction costs is ensured by having highly liquid ETFs and short ETFs on the S\&P 500.

Distributional regressions use the data sample to model a distribution of the dependent variable, conditional on the explanatory variable. In this way, the empirical distribution of the market return, conditional on the currently observed beta dispersion, can be determined. This approach has two major advantages compared to other approaches for modeling joint distributions (e.g., bivariate distributions or modeling a relation with copulas). First, it is not necessary to assume that the explanatory variable (here: beta dispersion) must have a specific distribution, and the distribution of the dependent variable (here: market return) can be flexibly tailored to the empirical observations. Second, the effective direction of the predictive relationship is studied in detail in the previous sections - the beta dispersion can to some extent predict market returns. This information can be used and empowers the modeling of the market return distribution via distributional regression. Beta is calculated based on backward-looking information, and the market return is estimated with forward-looking information. Therefore, it is reasonable to observe the beta dispersion and indicate the market return, and not the other way around. The alternative ways of bivariate modeling make no assumptions about the effective direction of a relationship between the variables, and this information is lost in these approaches.

The conditional probability $p$ of a positive market return can be determined by $p \mid B D=$ $1-F(0)$, where $F(0)$ is the cumulative distribution function of the conditional distribution of the market return. As mentioned, three different market timing strategies, using this probability as an investment trigger or better timing indicator, are introduced and studied. A probability higher than $50 \%$ for a positive market return in the next period indicates that 
the market will rise. First, a basic approach representing common market timing strategies is adopted from literature. Therefore, this strategy invests $100 \%$ in the market if the timing indicator signals rising markets. Otherwise, an investment in the money market is made. Second, an unweighted long-short market timing strategy, which either holds a $100 \%$ long position or a $100 \%$ short position in the market conditional on the timing indicator, is taken. Third, a weighted long-short strategy is adopted, which invests in a weighted market position proportional to the conditional probability of a positive market return. Formally, the weighted strategy holds a position of $X_{M}=2(p \mid B D-0.5)$ in the market and a position of $X_{R}=1-2(p \mid B D-0.5)$ in the money market. The transformation of the conditional probability for the weighed strategy ensures that the maximum investment that can be done in the market is 1 or $100 \%$ of the wealth and the minimum investment is -1 , which corresponds to a $100 \%$ short position in the market. All strategies start with an initial standardized wealth of 1 . This setting makes it easy to compare the market timing strategies with an appropriate benchmark.

In Chapter 1 benchmarks for market timing strategies depending on different risk conceptions are derived. Regarding risk factors, all risk is best reflected in the market portfolio. Consequently, a buy-and-hold strategy of the market portfolio for the entire sampled period is the appropriate benchmark. In addition, a commonly used 60/40 portfolio split between stocks and bonds ${ }^{8}$ is used as an additional benchmark, because the weighted strategy has an average weight from only around 0.5 in the market portfolio and hence this benchmark reflects the potential source of risk more appropriate. Applying regret as risk conception, the benchmark is the timing strategy that only invested in the market portfolio, when this return was higher than the risk-free return. This no-regret benchmark is only possible to determine ex-post. As performance measure the maximum drawdown is included, to cover this risk conception.

The analyses in the previous subsection showed that BD, based on beta dispersion estimated for 6, 12, and 36 months, is more valuable for long-term prediction (6- and 12-month market return). Every month, these specifications of the BD are used to estimate distributional

\footnotetext{
${ }^{8}$ This benchmark invests $60 \%$ of the wealth in the S\&P 500 Index and $40 \%$ of the wealth in the money market (Asness 1996; Leibowitz and Bova 2009).
} 
regressions over an extending window including all months prior to the month in which the portfolio is set up. It is assured that none of the strategies uses any in-sample information. The first conditional distribution can be determined in April 1984 to include a sufficient number of observations for the estimation. ${ }^{9}$ Depending on the forecasting horizon of the market return, the first market timing strategy is set up in October 1984 (6-month market return) and April 1985 (12-month market return). Irrespective of the prediction horizon, the position held in the market is rebalanced every month to adjust the weight in the market portfolio to the most current information.

\section{Figure 4.4: Weights of Market Timing Strategy}

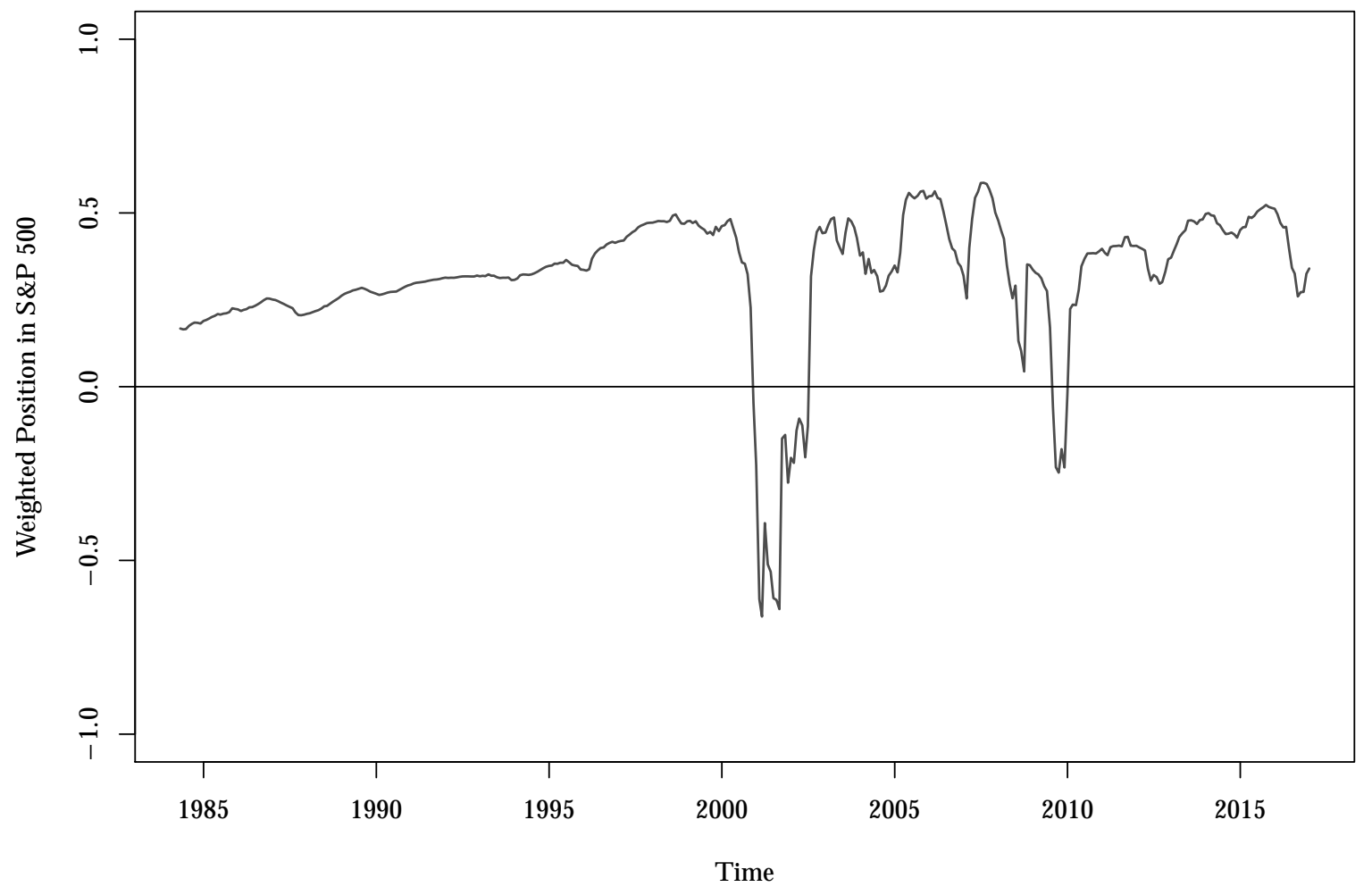

Note: Figure 4.4 shows the time series of the weights of the position held in the market (S\&P 500 Index) over the period April 1984 to December 2016. This period is shorter than the total sampled period because the first 20 years of the sample are used to have sufficient observations even for the first distributional regression. The weights are derived from the probability that the subsequent market return will be positive, calculated with distributional regressions. This probability is standardized between -1 and 1 , with $X_{M}=2(p \mid M-0.5)$. A weight of 1 means a $100 \%$ long position in the market and a weight of -1 implies a $100 \%$ short position in the market. The weights are based on the regression of the 12-month beta dispersion on the yearly market return.

\footnotetext{
${ }^{9}$ Compare the argumentation for the calculated out-of-sample $R_{O S}^{2}$ in Section 4.4.4.
} 
Figure 4.4 shows the development of the weights for the S\&P 500 Index, based on the distribution of the 12-month market return, conditional on the 12-month BD. The level of the weights is important only for the weighted strategy. The other two strategies are fully invested in the market when the weight is positive, and investments are done in the money market or short position in the market is taken, respectively, when the weight is negative. Notably, the conditional weight is only negative ${ }^{10}$ twice, namely, for the two most recent market downturns in the sample. After the results of Sections 4.4.2 and 4.4.3, this new perspective emphasizes the capability of the beta dispersion to serve as a measure of market vulnerability.

Figure 4.5 compares the time series of the increase in the total wealth of the three market timing approaches and corresponds to the weights shown in the Figure 4.4. The increase in total wealth is calculated by adjusting the weight held in the market portfolio every month and saving all earned returns in a money market account until the end of the sampled period in December 2016.

\footnotetext{
${ }^{10} \mathrm{~A}$ negative weight in the market corresponds to holding a short position in the market portfolio for the weighted- and the unweighted timing strategy.
} 


\section{Figure 4.5: Increase in Total Wealth Resulting from Timing Strategies}

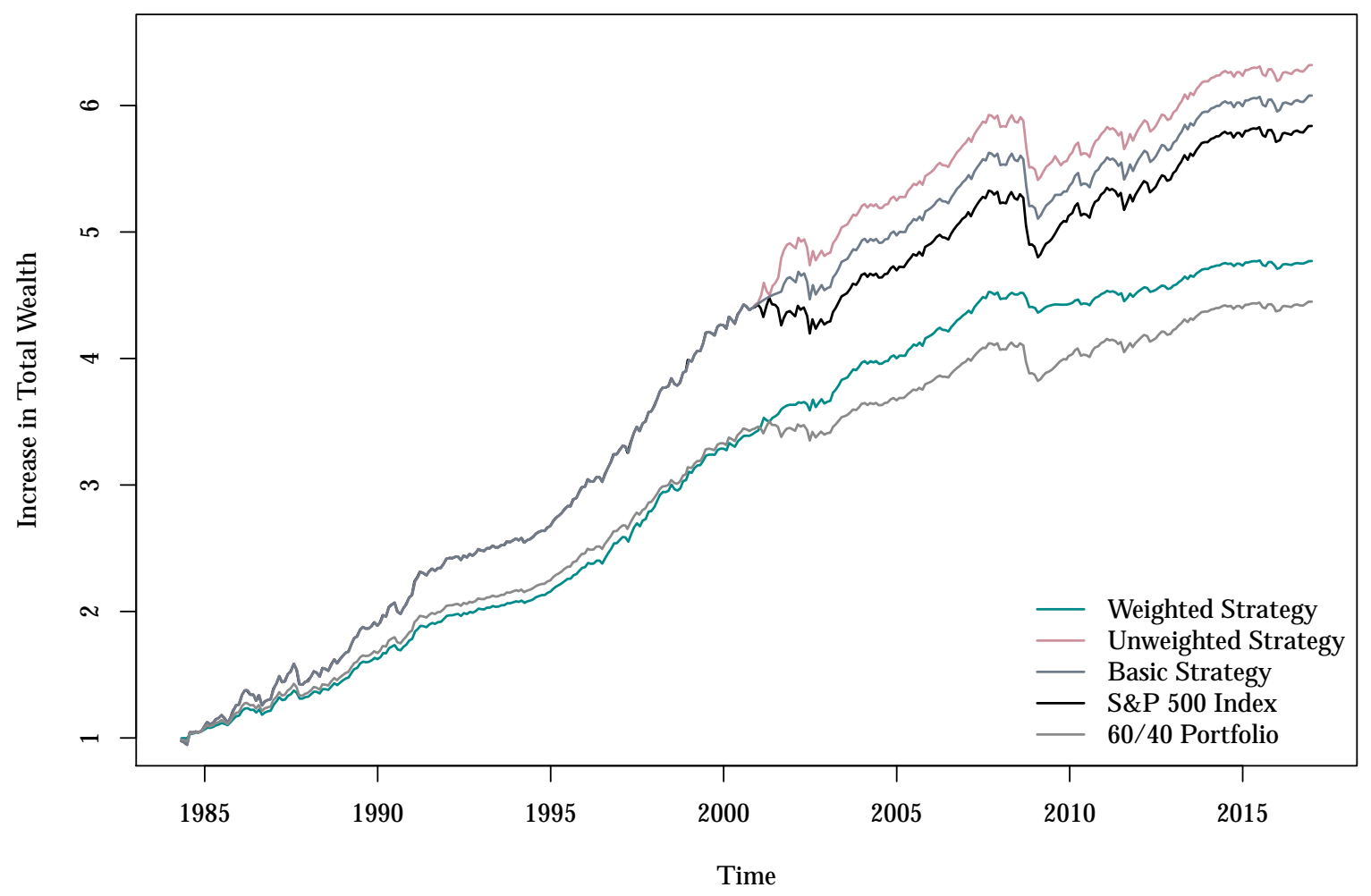

Note: Figure 4.5 shows an increase in the total wealth for the market timing strategies, based on the 12-month BD measure and a passive buy-and-hold strategy of the S\&P 500 and a 60/40 portfolio as benchmarks. The period from April 1984 to December 2016 is displayed. This period is shorter than the total sampled period because the first 20 years of the sample are used to have sufficient observations even for the first distributional regression. An increase in the total wealth comprises the currently earned return from the timing strategy as well as all previously earned returns accrued at the risk-free rate.

The basic and the unweighted strategy imitate the market until around 2001. This is the first time that the timing indicator foreshadows a market downturn. The basic strategy shifts the wealth from the market portfolio to a money market account, which is clearly visible in Figure 4.5. The unweighted strategy takes a short position in the market, and, therefore, can increase the total wealth for the investor more distinctly than the aforementioned strategy. The second market downturn is much less anticipated by these two strategies, and hence there is a decline in the performance. The weighted strategy falls short in terms of an absolute increase in wealth, compared to the buy-and-hold strategy and the other two timing strategies. The strength of this strategy is seen in the lasting reduction of return volatility in combination with only a slight decrease in return performance. Compared 
to the more appropriate $60 / 40$ benchmark $^{11}$, the weighted strategy is preferable. This is because the two large market downturns lead to much less performance loss than for the $60 / 40$ portfolio. Taking all together, the beta dispersion can successfully discriminate between market up- and downturns, and, therefore, it leads to a superior performance of timing strategies compared to the two chosen benchmarks, either in terms of absolute wealth or volatility reduction.

The described results shown in Figure 4.5 hold for all implemented variations of the market timing strategies, which can be seen in Table 4.7 .

\footnotetext{
${ }^{11}$ Compared to the buy-and-hold benchmark, this benchmark is more appropriate because the weighted strategy rarely invests $100 \%$ of the wealth in the market portfolio. The $60 / 40$ benchmark matches more or less with the mean investment of the weighted strategy in the market portfolio and, therefore, reflects the risk characteristics of the weighted strategy.
} 
Table 4.7: Performance of Market Timing Strategies

\begin{tabular}{|c|c|c|c|c|c|c|}
\hline & Distribution & al Regression & Av. Return & SD & SR & MDD \\
\hline & $R_{M}$ & $\mathrm{BD}$ & & & & \\
\hline & 6 Months & 6 Months & 0.1002 & 0.1348 & 0.5511 & -0.1043 \\
\hline 60 & 6 Months & 12 Months & 0.1060 & 0.1318 & 0.6080 & -0.1043 \\
\hline$\underset{\pi}{\overparen{\pi}}$ & 6 Months & 36 Months & 0.1107 & 0.1301 & 0.6512 & -0.1043 \\
\hline.$\circlearrowright$ & 12 Months & 6 Months & 0.1017 & 0.1346 & 0.5629 & -0.1043 \\
\hline صి & 12 Months & 12 Months & 0.1060 & 0.1318 & 0.6080 & -0.1043 \\
\hline & 12 Months & 36 Months & 0.1096 & 0.1303 & 0.6420 & -0.1043 \\
\hline 80 & 6 Months & 6 Months & 0.1005 & 0.1374 & 0.5425 & -0.1043 \\
\hline 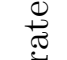 & 6 Months & 12 Months & 0.1121 & 0.1368 & 0.6302 & -0.1043 \\
\hline 泀 & 6 Months & 36 Months & 0.1214 & 0.1361 & 0.7006 & -0.1043 \\
\hline 类 & 12 Months & 6 Months & 0.1035 & 0.1373 & 0.5650 & -0.1043 \\
\hline$\frac{\infty}{0}$ & 12 Months & 12 Months & 0.1121 & 0.1368 & 0.6302 & -0.1043 \\
\hline 点 & 12 Months & 36 Months & 0.1192 & 0.1362 & 0.6844 & -0.1043 \\
\hline$b_{0}$ & 6 Months & 6 Months & 0.0551 & 0.0469 & 0.6216 & -0.0364 \\
\hline$\underset{\infty}{ \pm}$ & 6 Months & 12 Months & 0.0610 & 0.0494 & 0.7125 & -0.0332 \\
\hline$\underset{\pi}{\pi}$ & 6 Months & 36 Months & 0.0674 & 0.0547 & 0.7620 & -0.0376 \\
\hline 芯 & 12 Months & 6 Months & 0.0708 & 0.0670 & 0.6711 & -0.0385 \\
\hline.$\frac{.00}{0}$ & 12 Months & 12 Months & 0.0799 & 0.0721 & 0.7510 & -0.0466 \\
\hline 3 & 12 Months & 36 Months & 0.0853 & 0.0769 & 0.7754 & -0.0723 \\
\hline & Buy-and-ho & d Benchmark & 0.1000 & 0.1373 & 0.5386 & -0.1043 \\
\hline & $60 / 40 \mathrm{~B}$ & enchmark & 0.0705 & 0.0827 & 0.5386 & -0.0737 \\
\hline & No-regret & Benchmark & 0.2432 & 0.0754 & 2.0922 & 0.0000 \\
\hline
\end{tabular}

Note: Table 4.7 shows the average returns, standard deviations (SD), Sharpe ratios (SR), and the maximum drawdown (MDD) of the basic, unweighted, and weighted market timing strategies. In the last three rows, the figures of the three benchmark strategies (buy-and-hold, 60/40 portfolio split and no-regret) are presented. All values are annualized. The SR is calculated on the excess return of every strategy. The drawdown represents the relative difference between the backward-looking highest value of the total value compared to the current value at any point in time. The maximum drawdown presented in the table is the maximum of this quantity.

Table 4.7 reports the average return, standard deviation, Sharpe ratio, and maximum drawdown (MDD) of all strategies. Irrespective of the strategy, all the average returns are positive. The basic strategy does not seem preferable compared to the buy-and-hold 
benchmark, although the average return and standard deviation are slightly better and there is an improvement in the Sharpe ratio. Taking the costs for implementing and monitoring this strategy into account, the additional work does not seem to pay off. This also applies for the unweighted strategy. Contrarily, the weighted strategy has lower average returns, but the return volatility reduces sharply, and hence the Sharpe ratio is the most favorable. Even when comparing all described strategies to the $60 / 40$ benchmark, instead of the buy-and-hold benchmark, the weighted strategy provides the most interesting risk-return characteristics. The no-regret benchmark cannot be beaten by definition, nevertheless, the weighted strategy clearly nears this benchmark in terms of taken risk.

The drawdown is calculated as the relative difference between the backward-looking highest value of the total value to the current value at any point in time. ${ }^{12}$ In Table 4.7 the value MDD, which is the highest value in the time series of drawdowns, is presented for the entire investigation period of every strategy. The MDD can confirm the preferable characteristics of the weighted market timing strategy. The MDD of this strategy does not exceed its yearly average return, and this emphasizes that the weighted market timing strategy is relatively less risky. Of course the no-regret benchmark has a MDD of O. Nevertheless, the weighted strategies com closest to this value.

Overall, the most striking result of the market timing strategies is that, owing to the distributional regression approach, the weighted strategy reduces the standard deviation of the returns. ${ }^{13}$ This leads to outstanding Sharpe ratios, which are considerably higher than the Sharpe ratios of the benchmarks (buy-and-hold and 60/40). With risk reduction as a reasonable target of investment strategies, the results of the weighted market timing strategy should be highlighted as it reduces the standard deviation by up to $65 \%$ compared to the buy-and-hold benchmark and up to $20 \%$ compared to the $60 / 40$ benchmark. The use of the probability from the distributional regression as timing indicator is an innovative and successful way of improving the risk and return characteristics of market timing strategies. There seems to be a clear advantage in performance that arises from calculating the weights

\footnotetext{
${ }^{12}$ The drawdown is based on the risk conception of regret, described in Chapter 1 . The less negative the drawdown, the lower the regret for an investor of not being invested in the market in the right periods of time.

${ }^{13}$ Reduction of the standard deviation refers to the comparison of the standard deviation of the weighted strategy with the standard deviation of the benchmarks (buy-and-hold and 60/40).
} 
from the conditional distribution and adjusting this for the newest information of the beta dispersion by monthly rebalancing ${ }^{14}$.

Nevertheless, market timing strategies face some shortcomings that should be addressed. As per Zakamulin (2014), most market timing strategies lose their superior performance when realistic frictions are employed to the strategies. The most fundamental friction are liquidity, transaction costs, and prediction accuracy. The introduced strategies are all based on investments in the S\&P 500 Index, which represents a very actively traded market segment. Concerning this index, highly liquid ETFs exist that facilitate the easy implementation of the strategies; hence, concerns about liquidity are seemingly inapplicable in this case. Likewise, transaction costs can be expected to be low. As trading frequency also influences the cost of trading, the number of transactions for the basic and the unweighted strategy can be determined easily. The weight (compare Figure 4.4) changes two times in 32 years from positive to negative and the other way around. This means that the investor has to sell and rebuy in the market only twice, which seems justifiable. For the weighted market timing strategy, further analysis is necessary because this strategy has to be rebalanced every month. Therefore, the performance decline in terms of the Sharpe ratio is measured when the weights of the strategy are only rebalanced when the weight change exceeds specific limits, and hence the rebalancing frequency is reduced. Tests are conducted to examine how rarely the weight can be changed without the Sharpe ratio of the weighted strategy dropping below 0.60 , which is still clearly above both benchmarks' Sharpe ratio (buy-and-hold and 60/40). Until the weight is only rebalanced when the absolute change is greater than 25 percentage points, the Sharpe ratio stays above 0.60, but the trading activities drop to 15 to 30 times (compared to 390 times), depending on the exact specification of the distributional regression. This is less than once a year. It illustrates that the transaction costs can be reduced considerably without much decrease in performance and, especially, by not increasing the return volatility. The third shortcoming of timing strategies - predictive accuracy — is captured by the combination of the distributional regression and the weighted strategy. A complete wealth shift between stock and money market is of limited suitability, since the investor has to ensure that his prediction is

\footnotetext{
${ }^{14}$ Other rebalancing frequencies are tested, which lead to comparable results. Nevertheless, the presented findings for the monthly rebalancing are the most striking.
} 
sufficiently accurate (Sharpe 1975; Jeffrey 1984; Bauer Jr. and Dahlquist 2001; Neuhierl and Schlusche 2011; Hallerbach 2014). The weighted strategy can be considered as relying on less predictive accuracy. It is because this strategy decreases the weight in the market portfolio only gradually when the likelihood of a market downturn rises and vice versa. The more uncertain the prediction about the future market return, the lesser the total weight that is invested in the market. This ensures that if the indicator points in the wrong direction, the consequences (negative returns of the timing strategy) would be as small as possible. Hence, predictions that do not turn out to be true are not as harmful as strategies that involve a total wealth shift. The weighted strategy overcomes this shortcoming and benefits from its more careful investment approach.

\subsection{Conclusion}

This study derives the link between ex-ante betas and ex-post market return and shows that this is driven by an underlying economic relationship. The linkage can be explained by an appealing economic idea. The beta dispersion as a measure of market vulnerability, or, in other words, the probability and extent of a severe market decline, is defined and described. A high heterogeneity between betas in a market reduces the ability of that market to cope with systematic shocks. A high beta dispersion makes the market highly vulnerable, and the crash of high-beta companies is more likely to spill over to other firms, thereby increasing the overall financial distress risk, which can be interpreted as a second-round endogenous shock. It can be seen that there is an economic and statistical significance of the suitability of this concept, wherein the beta dispersion complements well-known predictor of the market return and adds to the accuracy of the prediction. By conducting additional empirical analyses, it is confirmed that the conception and argumentation of the beta dispersion seems to be applicable. Furthermore, the study presents an innovative way of setting up market timing strategies by conducting distributional regressions to determine the timing indicator. This way of modeling is newly introduced to finance and seems to be convincing, based on the performance of the market timing strategies. The careful investment approach of the weighted market timing strategy delivers a promising 
risk and return characteristics that coincide with addressing usual shortcoming of market timing strategies.

The comprehensive findings of the study can be valuable for different stakeholders. For investors, the improved accuracy of market risk premium prediction and the introduction of distributional regression to timing strategies can be worthwhile. Both can enhance the implementation and performance of market timing strategies. Particularly, the distributional regression approach can be extended to other predictors (macroeconomic as well as technical) to enhance the performance of such strategies. For supervisors monitoring the financial stability of a market, the beta dispersion might serve as an additional indicator for measuring the market's vulnerability. It can complement and extend the possibilities of measuring and quantifying systemic risk in the stock market.

While it is intuitive that a group of stocks with extremely large betas can indicate a higher likelihood of systemic problems in the following periods, we certainly need a better understanding of why the beta dispersion carries information about future market movements and how this information is processed in the market. Especially, an in-depth analysis of the cascading effect should be conducted along with a specific focus on the spillovers and contagion of high-beta stocks during a systematic shock. A link of the beta dispersion to systemic risk measures and financial stability can give interesting insights about market characteristics that favor crisis-prone developments. 


\section{A Appendix}

\section{A.1 Calculated Variables}

Some variables for the predictive regressions are calculated based on the price information of the S\&P 500 and all its constituents:

- Log return of the market for $1,3,6$, and 12 months:

$R_{M, t}=\ln \left(\frac{P_{t+s}}{P_{t}}\right)$,

where $P$ is the price of the market index at time $t$ and $s$ is $1,3,6$, and 12 months.

- Average variance following Pollet and Wilson (2010):

$A V_{t}=\sum_{j=1}^{N} w_{j, t} \hat{\sigma}_{j, t}^{2}$,

where $N$ is the number of stocks traded at the market, $w$ is the weight of the stock $j$ at time $t$, and $\hat{\sigma}_{j}^{2}$ is the estimated volatility of stock $j$.

- Average correlation following Pollet and Wilson (2010):

$A C_{t}=\sum_{j=1}^{N} \sum_{j \neq k} w_{j, t} w_{k, t} \hat{\rho}_{j k, t}$,

where $N$ is the number of stocks traded at a market, $w$ is the weight of the stock $j$ or $k$, respectively, at time $t$, and $\hat{\rho}_{j k}$ is the correlation coefficient between stock $j$ and stock $k$.

- Moving average following Neely et al. (2014):

$M A(2,12)=M A_{2, t}-M A_{12, t}$ where $M A_{T, t}=\left(\frac{1}{s}\right) \sum_{i=0}^{s-1} P_{t-i}$ for $T=2,12$,

where $s$ is the number of trading days in the following $\mathrm{T}$ months for which the average is calculated and $P$ is the price of the market index at time $t$.

- Return dispersion following Maio (2016):

$R D_{t}=\sqrt{\frac{1}{N} \sum_{i=1}^{N}\left(R_{i, t}-\bar{R}_{M, t}\right)^{2}}$,

where $N$ is the number of stocks traded at a market, $R_{i}$ is the discrete return of stock $i$, and $\bar{R}_{M, t}$ is the weighted mean of the returns of all stock at the market. 


\section{A.2 Linear Predictive Regressions with $B D_{V W}$ and $Q B D_{10 \%}$}

Table 4.A1: Linear Predictive Regressions with $B D_{V W}$

Panel A: 1-Month Market Returns

\begin{tabular}{|c|c|c|c|c|}
\hline & $B D_{V W}^{3 M}$ & $B D_{V W}^{6 M}$ & $B D_{V W}^{12 M}$ & $B D_{V W}^{36 M}$ \\
\hline Constant & $\begin{array}{l}\mathbf{0 . 0 1 3 3} \\
(0.0008)\end{array}$ & $\begin{array}{l}\mathbf{0 . 0 1 4 2} \\
(0.0003)\end{array}$ & $\begin{array}{l}\mathbf{0 . 0 1 5 6} \\
(0.0001)\end{array}$ & $\begin{array}{l}\mathbf{0 . 0 1 5 9} \\
(0.0005)\end{array}$ \\
\hline Coefficient & $\begin{array}{r}-\mathbf{0 . 0 1 7 1} \\
(0.0334)\end{array}$ & $\begin{array}{r}-\mathbf{0 . 0 2 3 6} \\
(0.0206)\end{array}$ & $\begin{array}{r}-\mathbf{0 . 0 3 1 1} \\
(0.0047)\end{array}$ & $\begin{array}{r}-\mathbf{0 . 0 3 6 7} \\
(0.0138)\end{array}$ \\
\hline$R_{a d j}^{2}$ & 0.0147 & 0.0138 & 0.0140 & 0.0118 \\
\hline
\end{tabular}

Panel B: 3-Month Market Returns

\begin{tabular}{lcccc}
\hline & $B D_{V W}^{3 M}$ & $B D_{V W}^{6 M}$ & $B D_{V W}^{12 M}$ & \multicolumn{1}{c}{$B D_{V W}^{36 M}$} \\
\hline Constant & $\mathbf{0 . 0 5 3 3}$ & $\mathbf{0 . 0 5 8 2}$ & $\mathbf{0 . 0 6 0 3}$ & $\mathbf{0 . 0 6 2 3}$ \\
& $(0.0000)$ & $(0.0001)$ & $(0.0002)$ & $(0.0008)$ \\
& & & & \\
Coefficient & $\mathbf{- 0 . 0 6 8 8}$ & $\mathbf{- 0 . 0 9 7 9}$ & $\mathbf{- 0 . 1 1 8 9}$ & $\mathbf{- 0 . 1 4 2 0}$ \\
& $(0.0067)$ & $(0.0067)$ & $(0.0075)$ & $(0.0175)$ \\
$R_{\text {adj }}^{2}$ & 0.0200 & 0.0197 & 0.0199 & 0.0190 \\
\hline
\end{tabular}

Panel C: 6-Month Market Returns

\begin{tabular}{lcccc}
\hline & $B D_{V W}^{3 M}$ & $B D_{V W}^{6 M}$ & $B D_{V W}^{12 M}$ & $B D_{V W}^{36 M}$ \\
\hline Constant & $\mathbf{0 . 0 9 3 2}$ & $\mathbf{0 . 1 0 7 2}$ & $\mathbf{0 . 1 0 8 5}$ & $\mathbf{0 . 1 0 8 7}$ \\
& $(0.0000)$ & $(0.0000)$ & $(0.0001)$ & $(0.0010)$ \\
& & & & \\
Coefficient & $\mathbf{- 0 . 1 2 0 5}$ & $\mathbf{- 0 . 1 8 6 0}$ & $\mathbf{- 0 . 2 1 7 4}$ & $\mathbf{- 0 . 2 4 7 5}$ \\
& $(0.0038)$ & $(0.0027)$ & $(0.0041)$ & $(0.0202)$ \\
$R_{\text {adj }}^{2}$ & 0.0207 & 0.0216 & 0.0209 & 0.0189 \\
\hline
\end{tabular}

Panel D: 12-Month Market Returns

\begin{tabular}{lcccc}
\hline & $B D_{V W}^{3 M}$ & $B D_{V W}^{6 M}$ & $B D_{V W}^{12 M}$ & \multicolumn{1}{c}{$B D_{V W}^{36 M}$} \\
\hline Constant & $\mathbf{0 . 1 5 9 7}$ & $\mathbf{0 . 1 8 3 2}$ & $\mathbf{0 . 1 8 8 0}$ & $\mathbf{0 . 1 8 1 6}$ \\
& $(0.0001)$ & $(0.0001)$ & $(0.0005)$ & $(0.0051)$ \\
& & & & \\
Coefficient & $\mathbf{- 0 . 1 9 6 2}$ & $\mathbf{- 0 . 3 0 4 4}$ & $\mathbf{- 0 . 3 6 2 6}$ & $\mathbf{- 0 . 3 8 9 7}$ \\
& $(0.0049)$ & $(0.0049)$ & $(0.0129)$ & $(0.0601)$ \\
$R_{a d j}^{2}$ & 0.0193 & 0.0201 & 0.0195 & 0.0166 \\
\hline
\end{tabular}

Note: Table 4.A1 shows the results of the predictive regressions, with the beta dispersion as independent variable and the 1-, 3-, 6-, and 12-month log excess return of the S\&P 500 Index (Panel A, Panel B, Panel $\mathrm{C}$, and Panel $\mathrm{D}$ ) as the dependent variable. $\mathrm{BD}$ is the cross-sectional value-weighted standard deviation of the individual stocks' betas (compare Equation 4.5). Beta is estimated from daily returns over a period of $3,6,12$, and 36 months. The adjusted $R_{a d j}^{2}$ of the predictive regressions are given in the last row of the table. Overlapping periods of the dependent variable are addressed by using the correction proposed by Britten-Jones et al. (2011). The calculations also use the Newey-West estimator with corresponding lags to account for heteroscedasticity and autocorrelation in the residuals. The p-value is given in parenthesis for every coefficient. Coefficients that are significant at least at a $10 \%$ level are printed in boldface. 
Table 4.A2: Linear Predictive Regressions with $Q B D_{10 \%}$

\begin{tabular}{|c|c|c|c|c|}
\hline & $Q B D_{10 \%}^{3 M}$ & $Q B D_{10 \%}^{6 M}$ & $Q B D_{10 \%}^{12 M}$ & $Q B D_{10 \%}^{36 M}$ \\
\hline Constant & $\begin{array}{l}\mathbf{0 . 0 2 4 5} \\
(0.0023)\end{array}$ & $\begin{array}{l}\mathbf{0 . 0 2 4 4} \\
(0.0038)\end{array}$ & $\begin{array}{l}\mathbf{0 . 0 2 5 6} \\
(0.0010)\end{array}$ & $\begin{array}{l}\mathbf{0 . 0 2 2 1} \\
(0.0170)\end{array}$ \\
\hline Coefficient & $\begin{array}{r}-\mathbf{0 . 0 0 9 5} \\
(0.0172)\end{array}$ & $\begin{array}{r}-\mathbf{0 . 0 1 0 7} \\
(0.0261)\end{array}$ & $\begin{array}{r}-\mathbf{0 . 0 1 2 3} \\
(0.0092)\end{array}$ & $\begin{array}{r}-\mathbf{0 . 0 1 1 0} \\
(0.0679)\end{array}$ \\
\hline$R_{a d j}^{2}$ & 0.0081 & 0.0076 & 0.0089 & 0.0044 \\
\hline
\end{tabular}

Panel B: 3-Month Market Returns

\begin{tabular}{lcccc}
\hline & $Q B D_{10 \%}^{3 M}$ & $Q B D_{10 \%}^{6 M}$ & $Q B D_{10 \%}^{12 M}$ & \multicolumn{1}{c}{$Q B D_{10 \%}^{36 M}$} \\
\hline Constant & $\mathbf{0 . 0 9 7 7}$ & $\mathbf{0 . 1 0 0 5}$ & $\mathbf{0 . 0 9 8 1}$ & $\mathbf{0 . 0 8 6 2}$ \\
& $(0.0004)$ & $(0.0016)$ & $(0.0018)$ & $(0.0193)$ \\
Coefficient & $\mathbf{- 0 . 0 3 8 0}$ & $\mathbf{- 0 . 0 4 2 5}$ & $\mathbf{- 0 . 0 4 6 7}$ & $\mathbf{- 0 . 0 5 6 9}$ \\
& $(0.0062)$ & $(0.0129)$ & $(0.0135)$ & $(0.0722)$ \\
$R_{a d j}^{2}$ & 0.0234 & 0.0221 & 0.0213 & 0.0171 \\
\hline
\end{tabular}

Panel C: 6-Month Market Returns

\begin{tabular}{lcccc}
\hline & $Q B D_{10 \%}^{3 M}$ & $Q B D_{10 \%}^{6 M}$ & $Q B D_{10 \%}^{12 M}$ & $Q B D_{10 \%}^{36 M}$ \\
\hline Constant & $\mathbf{0 . 1 6 4 9}$ & $\mathbf{0 . 1 7 9 0}$ & $\mathbf{0 . 1 5 7 3}$ & $\mathbf{0 . 1 4 0 1}$ \\
& $(0.0002)$ & $(0.0006)$ & $(0.0048)$ & $(0.0294)$ \\
Coefficient & $\mathbf{- 0 . 0 6 3 5}$ & $\mathbf{- 0 . 0 7 9 5}$ & $\mathbf{- 0 . 0 7 3 1}$ & -0.0673 \\
& $(0.0033)$ & $(0.0061)$ & $(0.0291)$ & $(0.1018)$ \\
$R_{a d j}^{2}$ & 0.0232 & 0.0235 & 0.0197 & 0.0160 \\
\hline
\end{tabular}

Panel D: 12-Month Market Returns

\begin{tabular}{lcccc}
\hline & $Q B D_{10 \%}^{3 M}$ & $Q B D_{10 \%}^{6 M}$ & $Q B D_{10 \%}^{12 M}$ & $Q B D_{10 \%}^{36 M}$ \\
\hline Constant & $\mathbf{0 . 2 3 0 2}$ & $\mathbf{0 . 2 3 6 4}$ & $\mathbf{0 . 2 0 8 0}$ & 0.1949 \\
& $(0.0045)$ & $(0.0124)$ & $(0.0382)$ & $(0.1078)$ \\
Coefficient & $\begin{array}{r}\mathbf{- 0 . 0 9 4 3} \\
(0.0375)\end{array}$ & $\mathbf{- 0 . 1 6 1 8}$ & -0.0850 & -0.0826 \\
& 0.0175 & $0.0695)$ & $(0.1517)$ & $(0.2819)$ \\
$R_{a d j}^{2}$ & & & 0.0149 & 0.0132 \\
\hline
\end{tabular}

Note: Table 4.A2 shows the results of the predictive regressions, with the beta dispersion as independent variable and the 1-, 3-, 6-, and 12-month log excess return of the S\&P 500 Index (Panel A, Panel B, Panel $\mathrm{C}$, and Panel D) as the dependent variable. BD is the difference between the mean beta of the high-beta quantile and the low-beta quantile (compare Equation 4.4). Beta is estimated from daily returns over a period of $3,6,12$, and 36 months. The adjusted $R_{a d j}^{2}$ of the predictive regressions are given in the last row of the table. Overlapping periods of the dependent variable are addressed by using the correction proposed by Britten-Jones et al. (2011). The calculations also use the Newey-West estimator with corresponding lags to account for heteroscedasticity and autocorrelation in the residuals. The p-value is given in parenthesis for every coefficient. Coefficients that are significant at least at a $10 \%$ level are printed in boldface. 


\section{A.3 Distributional Regressions}

The idea of structured additive distributional regressions is to aim explicitly at the complete distribution of the dependent variable and not only on the expected value of the dependent variable (Klein et al. 2015; Silbersdorff 2017). Conditional on the explanatory variable, a conventional linear regression model focuses on the description of the dependent variable by its expectation. Therefore, information about the dependent variable is lost. The structured additive distributional regression estimates all parameters of the conditional distribution of the dependent variable and, therefore, describes the relationship between dependent and explanatory variable in detail. Unlike quantile regressions, which are distribution-free, distributional regressions are a parametric yet flexible way of modeling a relationship. The assumption of the distribution of the dependent variable survives, and all parameters of its distribution are estimated regarding the covariates of the regression. Applied to this study, the assumption of a normal distribution for the log market return $\left(y_{i}\right)$ seems a reasonable starting point, thus $y_{i} \sim N\left(\mu_{i}, \sigma_{i}\right)$. In a conventional regression, only the expected value of the market return, conditional on the observed beta dispersion $\left(x_{i}\right)$, is estimated. Here, in addition, the variance of the market return, conditional on the observed beta dispersion, is estimated as well. Both parameters are estimated simultaneously via a back-fitting algorithm (Rigby and Stasinopoulos 2005; Stasinopoulos and Rigby 2007). This algorithm uses penalized likelihood estimation to obtain unbiased estimates of the expected value and the standard deviation of the normal distribution that characterizes the market return. First, for each instance $i$, the distribution parameters are described with the following equations:

$$
\begin{aligned}
& \mu_{i}=\beta_{0}^{\mu}+\beta_{1}^{\mu} x_{i} \\
& \sigma_{i}=\beta_{0}^{\sigma}+\beta_{1}^{\sigma} x_{i} .
\end{aligned}
$$

Equation 4.11 represents the mean of the conditional normal distribution of $y_{i}$, and Equation 4.12 represents the standard deviation of this distribution. The intention is to identify $\beta_{0}^{\mu}, \beta_{1}^{\mu}, \beta_{0}^{\sigma}$ and $\beta_{1}^{\sigma}$ such that the likelihood of obtaining the $y_{i}$ is maximized. To 
this end, these two equations are used to replace $\mu_{i}$ and $\sigma_{i}$ in the density function of the normal distribution for each instance $i$ :

$$
f_{y_{i}}(y)=\frac{1}{\sqrt{2 \pi\left(\beta_{0}^{\sigma}+\beta_{1}^{\sigma} x_{i}\right)}} e^{-\frac{1}{2}\left(\frac{y-\beta_{0}^{\mu}+\beta_{1}^{\mu} x_{i}}{\beta_{0}^{\sigma}+\beta_{1}^{\sigma} x_{i}}\right)^{2}} .
$$

Subsequently, the algorithm estimates the beta coefficients via penalized maximum likelihood in such a manner that, under the joint distribution, the observed market returns $\left(y_{i}\right)$ are the most likely outcomes. To ensure that the standard deviation is positive, $\sigma_{i}$ is replaced by $\log \left(\sigma_{i}\right)$ in the Equation 4.12. The estimation procedure leads to a response function of $y_{i}$ with the parameters $\mu_{i}$ and $\sigma_{i}$, which depend on the observed beta dispersion $\left(x_{i}\right)$ and the corresponding estimated coefficients $\left(\beta_{0}^{\mu}, \beta_{1}^{\mu}, \beta_{0}^{\sigma}\right.$, and $\left.\beta_{1}^{\sigma}\right)$. Subsequently, the distribution of the market return can be expressed by $y_{i} \sim N\left(\beta_{0}^{\mu}+\beta_{1}^{\mu} x_{i}, \exp \left(\beta_{0}^{\sigma}+\beta_{1}^{\sigma} x_{i}\right)\right)$. This distribution can be used to estimate the probability of the market return being positive, conditional on the observed beta dispersion, which is used as a trigger for the market timing strategies. 


\section{Conclusion}

The overall objective of this dissertation is to study and advance portfolio strategies with addressing classical and alternative benchmarks. The importance of a reasonable benchmark specification and its influences on portfolio strategies is expounded. The chapters of this dissertation highlight different aspects of designing and improving portfolio strategies and explicitly address different benchmark specifications and their impact. The insights are of interest to researchers, portfolio managers and even to supervisors of capital markets.

To start with, Chapter 1 describes the relevance and objective of the dissertation and, to establish a basis, develops a comprehensive systematization of benchmark specifications for the first time. Chapter 2 and Chapter 3 perform portfolio selection strategies and address the three introduced risk conceptions. It can be shown that it is important how a portfolio is set up exactly and that alternative risk conceptions can be integrated into portfolio selection to better reflect investor needs. Chapter 4 focuses on timing strategies and highlights that substantial improvements of timing strategies can be achieved by choosing a careful investment approach that reduces risk considerably. This chapter summarizes the three main chapters briefly, recaps the overall objective and major findings and concludes with an outlook for further research.

\section{Summary of Main Chapters}

Chapter 2 compares different approaches of designing a portfolio strategy that exploits the low-beta anomaly. A systematic analysis of the influence of various design elements on the performance of these strategies is performed. This chapter addresses how investment ideas based on exploiting market anomalies can be made concrete. The anomaly as such is well studied, but it remained an open question whether investors were able to benefit from the systematical mispricing or not. It can be shown that it matters how exactly the anomaly is exploited. Strategies overweighting low-beta stocks differ substantially from 
strategies overweighting high-beta stocks. In addition, the positive performance of the strategy seems to be driven by the positive premium of the low-beta stocks and not the negative premium of the high-beta stocks. This is a quite important finding for investors who can only take long positions because it allows them to also benefit from trading on the anomaly. Depending on the investible universe reflected in the benchmark, the portfolio strategies either can attain positive risk-adjusted excess return (large investible universe) or their return is driven by unwittingly taking on other risks, such as market or size risk (smaller investible universe). Furthermore, it can be shown that design elements such as the estimation method of beta and the weighting of stocks within the portfolio have a large impact on the risk and return characteristics of the resulting strategies. The study reveals the importance of a reflected portfolio design. The explicit design of the strategy and modifications of design elements should be studied comprehensively to prevent investors from being exposed to risk and return characteristics that do not match their preferences. Chapter 3 has the purpose to integrate regret risk into portfolio selection following Markowitz $(1952,1987)$. Avoiding regret - caused by not choosing the ex-post best asset - is understood as an independent investor need in addition to final wealth. In contrast to Chapter 2, the contribution of this study is more conceptional. Nevertheless, implications for practical uses in portfolio management can be derived from the findings. The chapter shows that regret risk can be smoothly integrated into the portfolio selection approach. In this way, optimizing a portfolio for a risk- and regret-averse investor is as easy as for an investor who is risk-averse only. A simulation and the empirical study reveal that depending on the characteristics of the investible universe and its constituents, the portfolio weights can be more or less concentrated than for a solely risk-averse investor. The appropriate benchmark for regret risk is the ex-post best asset and obviously this benchmark is strongly dependent on the investible universe. It is impossible for the investor to outperform this benchmark, but the empirical study shows that the investor is able to minimize his expected regret. Applying more realistic assumptions to the construction of portfolio strategies by considering more than one investor need, namely final wealth and avoidance of regret, contributes to designing more suitable portfolio strategies that match 
the preferences and expectations of the investor more closely than by just relying on risk alone.

Chapter 4 differs from the other two chapters by approaching timing strategies instead of selection strategies. Because all risk is inherent in the factor portfolio that is going to be timed, the identification of an appropriate benchmark is much more straightforward than for selection strategies and the investible universe - a huge impact factor for selection strategies - fades into the background for timing strategies. The different risk conceptions are used to adjust the benchmark and evaluate the timing strategies accordingly. The main challenge is the derivation of a reasonable and applicable timing indicator. Such a comprehensive analysis is demonstrated in Chapter 4, by introducing and studying the beta dispersion as a measure of market vulnerability. This measure contributes to the accuracy of market return prediction by capturing a yet unregarded facet of systemic risk. The innovative approach of translating a predictor of the market return, here the beta dispersion, into a probability distribution of the future market return with the help of distributional regressions, shows how timing strategies can be improved. In combination with the weighted investment approach the portfolio strategy is clearly able to reduce the return volatility and is more favorable than the benchmarks. With risk reduction being a reasonable objective in portfolio management, this chapter demonstrates how this can be established and implemented with timing strategies.

\section{Contribution and Core Findings}

This thesis contributes to the advancement and understanding of portfolio strategies and their performance. The awareness for and the importance of a well-conceived portfolio design combined with a suitable benchmark specification is clearly highlighted and strengthened. Beyond that, for the first time, a systematization for benchmark specifications is offered and it is shown how an alternative benchmark, the ex-post best asset, can be applied to portfolio selection to enable more suitable investment decisions. The thesis directs the attention to the impact of the benchmark specification on portfolio strategies and consequences for investors. It is emphasized that implications for portfolio strategies, their 
design and their performance should be carefully drawn as they are sensitive to the exact design as well as the benchmark specification.

The findings of the thesis can be summarized into five core insights: (1) The performance of a portfolio strategy is sensitive to the exact specification and various design elements. (2) The investible universe as an element of the benchmark specification has a major impact on the design and performance of the implemented portfolio strategy. (3) Regret can be integrated into the portfolio selection as an alternative and additional risk conception that results in clearly deviating portfolio compositions compared to a portfolio optimized for a risk-averse investor solely. (4) The beta dispersion is introduced as a novel measure of market vulnerability. (5) The performance of timing strategies can be enhanced by choosing a careful investment approach based on distributional regressions and partially invested wealth.

These findings are of interest in research as well as in portfolio management. For both, these findings emphasize the importance of comparing the desired characteristics and objectives of a portfolio strategy with the actual outcome in terms of risk-return characteristics. Likewise, the findings accentuate that the suitability and the impact of the benchmark specification and inherent risk conception should be considered. Furthermore, integrating alternative risk conceptions into portfolio selection and considering two independent additional investor needs is enlightening. The integration of regret into portfolio optimization can help to construct portfolios that reflect investors' preferences even better and in doing so add value to them. Moreover, deriving the beta dispersion as a market vulnerability measure can be of great use for research and portfolio management but also for supervisors concerned about market stability. Introducing distributional regressions to finance is a clear gain of knowledge, which can be very useful not only for timing strategies, but also for modeling dependencies of variables in other contexts. Overall, the thesis emphasizes that the exact design of a portfolio strategy and its implications as well as the choice of an appropriate and corresponding benchmark should be well though out and not disregarded by researchers or practitioners. 


\section{Outlook}

Despite this thesis' multiple contributions, further research is still needed in several areas. A systematic analysis of the influences of different design elements on portfolio strategies exploiting anomalies, as performed in Chapter 2, could be extended to other anomalies. This could shed further light on whether and how market anomalies can be actually exploited by investors. Demonstrating how regret can be integrated into portfolio selection (Chapter 3) leads to further open questions: Can higher moments of the return distribution be integrated as well? Can other independent investor needs (e.g. avoidance of downside risk) likewise be integrated into portfolio selection? What are the consequences of extending the investible universe to non-linear instruments? Regarding the beta dispersion as measure of market vulnerability, Chapter 4 shows the applicability of the economic idea. To be a valuable tool for supervisors, further analysis on the precise evolvement of the cascading effect is needed. Furthermore, the timing approach can be translated to other indicators as well as different factor portfolios. In addition, the multiple distributional regressions have plenty possible applications in finance - further improvement of timing strategies is only one of them.

Taken together, research as conducted in this dissertation can help to shed light on the myriads of possibilities to set up portfolio strategies. By systematizing, studying, improving and advancing portfolio strategies and influences on it, investors should be better able to assess their preferences and objectives, achieve their desired outcome and make better, more suitable decision. 


\section{Bibliography}

Amenc, N., F. Ducoulombier, F. Goltz, A. Lodh, and S. Sivasubramanian (2016). Diversified or concentrated factor tilts. Journal of Portfolio Management 42(2), 64-76.

Amenc, N., F. Goltz, and A. Lodh (2012). Choose your betas: Benchmarking alternative equity index strategies. Journal of Portfolio Management 39(1), 88-111.

Amenc, N., F. Goltz, and A. Lodh (2016). Smart beta is not monkey business. Journal of Index Investing 6(4), 12-29.

Andersen, T. G., T. Bollerslev, F. X. Diebold, and J. G. Wu (2005). A framework for exploring the macroeconomic determinants of systematic risk. American Economic Review 95, 398-404.

Ang, A. and G. Bekaert (2007). Stock return predictability: Is it there? Review of Financial Studies 20(3), 651-707.

Ang, A., G. Bekaert, and J. Liu (2005). Why stocks may disappoint. Journal of Financial Economics 76(3), 471-508.

Ang, A., R. J. Hodrick, Y. Xing, and X. Zhang (2006). The cross section of volatility and expected returns. Journal of Finance 61(1), 259-299.

Ang, A., R. J. Hodrick, Y. Xing, and X. Zhang (2009). High idiosyncratic volatility and low returns: International and further U.S. evidence. Journal of Financial Economics $91(1)$, $1-23$.

Angelidis, T., A. Sakkas, and N. Tessaromatis (2015). Stock market dispersion, the business cycle and expected factor returns. Journal of Banking ES Finance 59, 265-279.

Asness, C. S. (1996). Why not 100\% equities. Journal of Portfolio Management 22(2), $29-34$. 
Auer, B. R. and F. Schuhmacher (2015). Liquid betting against beta in Dow Jones Industrial Average stocks. Financial Analysts Journal 76(6), 30-43.

Avdis, E. and J. A. Wachter (2017). Maximum likelihood estimation of the equity premium. Journal of Financial Economics 125(3), 589-609.

Avramov, D. and T. Chordia (2006). Predicting stock returns. Journal of Financial Economics 82(2), 387-415.

Baetje, F. and L. Menkhoff (2016). Equity premium prediction: Are economic and technical indicators unstable? International Journal of Forecasting 32(4), 1193-1207.

Baker, M., B. Bradley, and J. Wurgler (2011). Benchmarks as limits to arbitrage: Understanding the low-volatility anomaly. Financial Analysts Journal 67(1), 40-54.

Baker, N. L. and R. A. Haugen (2012). Low risk stocks outperform within all observable markets of the world. Working Paper, Guggenheim Investments.

Bali, T. G., S. J. Brown, S. Murray, and Y. Tang (2017). A lottery-demand-based explanation of the beta anomaly. Journal of Financial and Quantitative Analysis 52(6), 2369-2397.

Bali, T. G., R. F. Engle, and Y. Tang (2016). Dynamic conditional beta is alive and well in the cross section of daily stock returns. Management Science 63(11), 3760-3779.

Bauer Jr., R. J. and J. R. Dahlquist (2001). Market timing and roulette wheels. Financial Analysts Journal 57(1), 28-40.

Baule, R., O. Korn, and L.-C. Kuntz (2018). Markowitz with regret. Working Paper, University of Göttingen and University of Hagen.

Bekaert, G. and C. R. Harvey (2000). Foreign speculators and emerging equity markets. Journal of Finance 55(2), 565-613.

Bell, D. E. (1982). Regret in decision making under uncertainty. Operations Research 30(5), 961-981. 
Bell, D. E. (1983). Risk premiums for decision regret. Management Science 29(10), 1156-1166.

Berrada, T. and J. Hugonnier (2013). Incomplete information, idiosyncratic volatility and stock returns. Journal of Banking \& Finance 37, 448-462.

Best, M. J. and R. R. Grauer (1991). On the sensitivity of mean-variance-efficient portfolios to changes in asset means: Some analytical and computational results. Review of Financial Studies 4 (2), 315-342.

Bigelow, J. P. (1993). Consistency of mean-variance analysis and expected utility analysis. Economics Letters 43, 187-192.

Black, F. (1993). Beta and return. Journal of Portfolio Management 20(1), 74-84.

Black, F., M. C. Jensen, and M. S. Scholes (1972). The capital asset pricing model: Some empirical tests. In M. C. Jensen (Ed.), Studies in the Theory of Capital Markets, pp. 79-121. Praeger: New York.

Bleichrodt, H., A. Cillo, and E. Diecidue (2010). A quantitative measurement of regret theory. Management Science 56(1), 161-175.

Blitz, D. (2014). Agency-based asset pricing and the beta anomaly. European Financial Management 20(4), 770-801.

Blitz, D. (2016). The value of low volatility. Journal of Portfolio Management 42(3), 94-100.

Blitz, D., E. G. Falkenstein, and P. van Vliet (2014). Explanations for the volatility effect: An overview based on the CAPM assumptions. Journal of Portfolio Management 40(3), 61-76.

Blitz, D., J. Huij, S. D. Lansdorp, and P. van Vliet (2014). Factor investing: Long-only versus long-short. Working Paper, Erasmus University Rotterdam.

Blitz, D., J. Pang, and P. van Vliet (2013). The volatility effect in emerging markets. Emerging Markets Review 16, 31-45. 
Bollerslev, T., J. Marrone, L. Xu, and H. Zhou (2014). Stock return predictability and variance risk premia: Statistical inference and international evidence. Journal of Financial and Quantitative Analysis 49(3), 633-661.

Bollerslev, T., G. Tauchen, and H. Zhou. (2009). Expected stock returns and variance risk premia. Review of Financial Studies 22(11), 4463-4492.

Britten-Jones, M., A. Neuberger, and I. Nolte (2011). Improved inference in regression with overlapping observations. Journal of Business Finance \& Accounting 38 (5-6), 657-683.

Brock, W., J. Lakonishok, and B. LeBaron (1992). Simple technical trading rules and the stochastic properties of stock returns. Journal of Finance 47(5), 1731-1764.

Campbell, J. Y. and S. B. Thompson (2008). Predicting excess stock returns out of sample: Can anything beat the historical average? Review of Financial Studies 21(4), 1509-1531.

Carhart, M. M. (1997). On persistence in mutual fund performance. Journal of Finance 52(1), 57-82.

Cederburg, S. and M. S. O'Doherty (2016). Does it pay to bet against beta? On the conditional performance of the beta anomaly. Journal of Finance 71(2), 737-774.

Chen, S.-S. (2009). Predicting the bear stock market: Macroeconomic variables as leading indicators. Journal of Banking 8 Finance 33(2), 211-223.

Chopra, V. K. and W. T. Ziemba (1993). The effect of errors in means, variances, and covariances on optimal portfolio choice. Journal of Portfolio Management 19(2), 6-11.

Chow, T.-M., J. C. Hsu, L.-L. Kuo, and F. Li (2014). A study of low-volatility portfolio construction methods. Journal of Portfolio Management 40(4), 89-105.

Christie, W. G. and R. D. Huang (1995). Following the pied piper: Do individual returns herd around the market? Financial Analysts Journal 51(4), 31-37.

Christoffersen, S. E. and M. Simutin (2017). On the demand for high-beta stocks: Evidence from mutual funds. Review of Financial Studies 30(8), 2596-2620. 
Clark, T. E. and K. D. West (2007). Approximately normal tests for equal predictive accuracy in nested models. Journal of Econometrics 138(1), 291-311.

Cochrane, J. H. (2008). The dog that did not bark: A defense of return predictability. Review of Financial Studies 21(4), 1533-1575.

Connolly, R. and C. Stivers (2003). Momentum and reversals in equity-index returns during periods of abnormal turnover and return dispersion. Journal of Finance 58(4), $1521-1556$.

Conrad, J., R. F. Dittmar, and E. Ghysels (2013). Ex ante skewness and expected stock returns. Journal of Finance 68(1), 85-124.

Constantinides, G. M. (2002). Rational asset prices. Journal of Finance 57(4), 1567-1591.

Das, S. R., H. M. Markowitz, J. Scheid, and M. Statman (2010). Portfolio optimization and mental accounts. Journal of Financial and Quantitative Analysis 45(2), 311-334.

Das, S. R. and M. Statman (2013). Options and structured products in behavioral portfolios. Journal of Economic Dynamics \& Control 37, 137-153.

DeMiguel, V., L. Garlappi, F. J. Nogales, and R. Uppal (2009). A generalized approach to portfolio optimization: Improving performance by constraining portfolio norms. Management Science 55(5), 798-812.

Dichtl, H., W. Drobetz, and L. Kryzanowski (2016). Timing the stock market: Does it really make no sense? Journal of Behavioral and Experimental Finance 10, 88-104.

Diecidue, E. and J. Somasundaram (2017). Regret theory: A new foundation. Journal of Economic Theory 172, 88-119.

Dimson, E. (1979). Risk measurement when shares are subject to infrequent trading. Journal of Financial Economics 7(2), 197-226.

Donaldson, R. G., M. J. Kamstra, and L. A. Kramer (2010). Estimating the equity premium. Journal of Financial and Quantitative Analysis 45(4), 813-846. 
Dor, A. B., V. Budinger, L. Dynkin, and K. Leech (2008). Constructing peer benchmarks for mutual funds: A style analysis-based approach. Journal of Portfolio Management $34(2)$, 65.

Driessen, J. and P. Maenhout (2007). An empirical portfolio perspective on option pricing anomalies. Review of Finance 11(4), 561-603.

Dutt, T. and M. Humphery-Jenner (2013). Stock return volatility, operating performance and stock returns: International evidence on drivers of the low volatility anomaly. Journal of Banking \& Finance 37, 999-1017.

Faff, R. W., D. Hillier, and J. Hillier (2000). Time varying beta risk: An analysis of alternative modelling techniques. Journal of Business Finance 8 Accounting 27(5-6), $523-554$.

Fama, E. F. (1970). Efficient capital markets: A review of theory and empirical work. Journal of Finance 25(2), 383-417.

Fama, E. F. (1991). Efficient capital markets: II. Journal of Finance 46 (5), 1575-1617.

Fama, E. F. and K. R. French (1992). The cross-section of expected stock returns. Journal of Finance $47(2), 427-465$.

Fama, E. F. and K. R. French (1993). Common risk factors in the returns on stocks and bonds. Journal of Financial Economics 33(1), 3-56.

Feldman, T., A. Jung, and J. Klein (2015). Buy and hold versus timing strategies: The winner is... Journal of Portfolio Management 42(1), 110-118.

Ferson, W. E. and C. R. Harvey (1993). The risk and predictability of international equity returns. Review of Financial Studies 6(3), 527-566.

Frazzini, A. and L. H. Pedersen (2014). Betting against beta. Journal of Financial Economics 111(1), 1-25.

Frost, P. A. and J. E. Savarino (1988). For better performance: Constrain portfolio weights. Journal of Portfolio Management 15(1), 29-34. 
Frydman, C. and C. Camerer (2016). Neural evidence of regret and its implications for investor behavior. Review of Financial Studies 29(11), 3108-3139.

Gollier, C. and B. Salanié (2006). Individual decisions under risk, risk sharing and asset prices with regret. Working Paper, University of Toulouse.

Goyal, A. and I. Welch (2003). Predicting the equity premium with dividend ratios. Management Science 49(5), 639-654.

Grundy, B. D. and J. S. M. Martin (2001). Understanding the nature of the risks and the source of the rewards to momentum investing. Review of Financial Studies 14(1), 29-78.

Hallerbach, W. G. (2014). On the expected performance of market timing strategies. Journal of Portfolio Management 40(4), 42-51.

Hansen, L. P. and S. F. Richard (1987). The role of conditioning information in deducing testable restrictions implied by dynamic asset pricing models. Econometrica: Journal of the Econometric Society, 587-613.

Hansen, P. R. and A. Timmermann (2012). Choice of sample split in out-of-sample forecast evaluation. Working Paper, European University Institute Florence.

Harlow, W. (1991). Asset allocation in a downside-risk framework. Financial Analysts Journal 47(5), 28-40.

Haugen, R. A. and A. J. Heins (1975). Risk and the rate of return on financial assets: Some old wine in new bottles. Journal of Financial and Quantitative Analysis 10(5), 775-784.

Hollstein, F. and M. Prokopczuk (2016). Estimating beta. Journal of Financial and Quantitative Analysis 51(4), 1437-1466.

Hong, H. and D. A. Sraer (2016). Speculative betas. Journal of Finance 71(5), 2095-2144.

Hsu, J. (2014). Value investing: Smart beta vs. style indices. Journal of Indexes 5(1), 121-126. 
Hunter, D., E. Kandel, S. Kandel, and R. Wermers (2014). Mutual fund performance evaluation with active peer benchmarks. Journal of Financial Economics 112(1), 1-29.

Ibbotson, R. G. and P. Chen (2003). Long-run stock returns: Participating in the real economy. Financial Analysts Journal 59(1), 88-98.

Jacobs, B. I. and K. N. Levy (2014). Smart beta versus smart alpha. Journal of Portfolio Management 40(4), 4-7.

Jacobs, H. (2015). What explains the dynamics of 100 anomalies? Journal of Banking \& Finance 57, 65-85.

Jacobs, H. (2016). Beta and biased beliefs. Working Paper, University of Mannheim.

Jagannathan, R. and T. Ma (2003). Risk reduction in large portfolios: Why imposing the wrong constraints helps. Journal of Finance 58(4), 1651-1684.

Jagannathan, R. and Z. Wang (1996). The conditional CAPM and the cross-section of expected returns. Journal of Finance 51(1), 3-53.

Jank, S. and E. Smajlbegovic (2016). Dissecting short-sale performance: Evidence from large position disclosures. Working Paper, University of Mannheim.

Jeffrey, R. H. (1984). The folly of stock-market timing. Harvard Business Review 62(4), $102-110$.

Jensen, M. C. (1969). Risk, the pricing of capital assets, and the evaluation of investment portfolios. Journal of Business 42(2), 167-247.

Jiang, X. (2010). Return dispersion and expected returns. Financial Markets and Portfolio Management 24(2), 107-135.

Jondeau, E. and M. Rockinger (2001). Gram-charlier densities. Journal of Economic Dynamics \& Control 25, 1457-1483.

Jostova, G. and A. Philipov (2005). Bayesian analysis of stochastic betas. Journal of Financial and Quantitative Analysis 40(04), 747-778. 
Jylhä, P., M. Suominen, and T. Tomunen (2017). Beta bubbles. Review of Asset Pricing Studies, $\operatorname{rax} 014$.

Klebaner, F., Z. Landsman, U. Makov, and J. Yao (2017). Optimal portfolios with downside risk. Quantitative Finance 17(3), 315-325.

Klein, N., T. Kneib, S. Lang, and A. Sohn (2015). Bayesian structured additive distributional regression with an application to regional income inequality in Germany. Annals of Applied Statistics 9(2), 1024-1052.

Korn, O. and L.-C. Kuntz (2018). Low-beta strategies. Working Paper, University of Göttingen.

Korn, O. and M. O. Rieger (2017). Hedging with regret. Working Paper, University of Göttingen and University of Trier.

Kostakis, A., N. Panigirtzoglou, and G. Skiadopoulos (2011). Market timing with optionimplied distributions: A forward-looking approach. Management Science 57(7), 12311249.

Kroll, Y., H. Levy, and H. M. Markowitz (1984). Mean-variance versus direct utility maximization. Journal of Finance 39(1), 47-61.

Kuntz, L.-C. (2018). Beta dispersion and market timing. Working Paper, University of Göttingen.

Ledoit, O. and M. Wolf (2004). Honey, I shrunk the sample covariance matrix. Journal of Portfolio Management 30(4), 110-119.

Leibowitz, M. L. and A. Bova (2009). Diversification performance and stress-betas. Journal of Portfolio Management 35(3), 41.

Lettau, M. and S. Ludvigson (2001). Consumption, aggregate wealth and expected stock returns. Journal of Finance 56(3), 815-849.

Levy, H. and H. M. Markowitz (1979). Approximating expected utility by a function of mean and variance. American Economic Review 69(3), 308-317. 
Lewellen, J. (2004). Predicting returns with financial ratios. Journal of Financial Economics 74 (2), 209-235.

Lewellen, J. and S. Nagel (2006). The conditional CAPM does not explain asset-pricing anomalies. Journal of Financial Economics 82(2), 289-314.

Li, X., R. N. Sullivan, and L. Garcia-Feijoo (2014). The limits to arbitrage and the low-volatility anomaly. Financial Analysts Journal 70(1), 52-63.

Lintner, J. (1965). The valuation of risk assets and the selection of risky investments in stock portfolios and capital budgets. Review of Economics and Statistics, 13-37.

Loomes, G. and R. Sugden (1982). Regret theory: An alternative theory of rational choice under uncertainty. Economic Journal 92, 805-824.

Maio, P. (2016). Cross-sectional return dispersion and the equity premium. Journal of Financial Markets 29, 87-109.

Malkiel, B. G. (2014). Is smart beta really smart? Journal of Portfolio Management 40(5), $127-134$.

Markowitz, H. M. (1952). Portfolio selection. Journal of Finance 7(1), 77-91.

Markowitz, H. M. (1987). Mean-Variance Analysis in Portfolio Choice and Capital Markets. John Wiley \& Sons.

Martin, I. (2017). What is the expected return on the market? Quarterly Journal of Economics 132(1), 367-433.

Merton, R. C. (1980). On estimating the expected return on the market: An exploratory investigation. Journal of Financial Economics 8(4), 323-361.

Meyer, J. (1987). Two-moment decision models and expected utility maximization. American Economic Review $77(3), 421-430$.

Michenaud, S. and B. Solnik (2008). Applying regret theory to investment choices: Currency hedging decisions. Journal of International Money and Finance 27(5), 677-694. 
Mossin, J. (1966). Equilibrium in a capital asset market. Econometrica: Journal of the Econometric Society, $768-783$.

Muermann, A., O. S. Mitchell, and J. M. Volkman (2006). Regret, portfolio choice, and guarantees in defined contribution schemes. Insurance: Mathematics and Economics 32(2), 219-229.

Neely, C. J., D. E. Rapach, J. Tu, and G. Zhou (2014). Forecasting the equity risk premium: The role of technical indicators. Management Science 60(7), 1772-1791.

Neuhierl, A. and B. Schlusche (2011). Data snooping and market-timing rule performance. Journal of Financial Econometrics 9(3), 550-587.

Pfeifer, P. E. (1985). Market timing and risk reduction. Journal of Financial and Quantitative Analysis 20(04), 451-459.

Pollet, J. M. and M. Wilson (2010). Average correlation and stock market returns. Journal of Financial Economics 96(3), 364-380.

Quaranta, A. G. and A. Zaffaroni (2008). Robust optimization of conditional value at risk and portfolio selection. Journal of Banking \& Finance 32(10), 2046-2056.

Rapach, D. E., J. K. Strauss, and G. Zhou (2010). Out-of-sample equity premium prediction: Combination forecasts and links to the real economy. Review of Financial Studies 23(2), $821-862$.

Rapach, D. E. and G. Zhou (2013). Forecasting stock returns. In G. Elliott and A. Timmermann (Eds.), Handbook of Economic Forecasting, Volume 2, pp. 328-383. Elsevier: Amsterdam.

Reeves, J. J. and H. Wu (2013). Constant versus time-varying beta models: Further forecast evaluation. Journal of Forecasting 32(3), 256-266.

Rigby, R. A. and D. M. Stasinopoulos (2005). Generalized additive models for location, scale and shape. Journal of the Royal Statistical Society: Series C (Applied Statistics) 54(3), $507-554$. 
Rouwenhorst, K. G. (1999). Local return factors and turnover in emerging stock markets. Journal of Finance 54(4), 1439-1464.

Schneider, P., C. Wagner, and J. Zechner (2016). Low risk anomalies? Working Paper, Swiss Finance Institute.

Sharpe, W. F. (1964). Capital asset prices: A theory of market equilibrium under conditions of risk. Journal of Finance 19(3), 425-442.

Sharpe, W. F. (1966). Mutual fund performance. Journal of Business 39(1), 119-138.

Sharpe, W. F. (1975). Likely gains from market timing. Financial Analysts Journal 31(2), 60-69.

Sharpe, W. F. (1994). The Sharpe ratio. Journal of Portfolio Management 21(1), 49-58.

Shefrin, H. and M. Statman (2000). Behavioral portfolio theory. Journal of Financial and Quantitative Analysis 35(2), 127-151.

Shen, P. (2003). Market timing strategies that worked. Journal of Portfolio Management 29(2), 57-68.

Shi, Y., X. Cui, and D. Li (2015). Discrete-time behavioral portfolio selection under cumulative prospect theory. Journal of Economic Dynamics \& Control 61, 283-302.

Silbersdorff, A. (2017). Estimating and Assessing Distributional Regression. Springer.

Spiegel, M. (2008). Forecasting the equity premium: Where we stand today. Review of Financial Studies 21(4), 1453-1454.

Stambaugh, R. F. (1999). Predictive regressions. Journal of Financial Economics 54(3), $375-421$.

Stasinopoulos, D. M. and R. A. Rigby (2007). Generalized additive models for location scale and shape (GAMLSS) in R. Journal of Statistical Software 23(7), 1-46.

Stivers, C. T. (2003). Firm-level return dispersion and the future volatility of aggregate stock market returns. Journal of Financial Markets 6(3), 389-411. 
Sugden, R. (1993). An axiomatic foundation for regret theory. Journal of Economic Theory 60, 159-180.

Wang, K. Q. (2003). Asset pricing with conditioning information: A new test. Journal of Finance 58(1), 161-196.

Welch, I. and A. Goyal (2008). A comprehensive look at the empirical performance of equity premium prediction. Review of Financial Studies 21(4), 1455-1508.

Whaley, R. E. (2009). Understanding the VIX. Journal of Portfolio Management 35(3), 98-105.

Zakamulin, V. (2014). The real-life performance of market timing with moving average and time-series momentum rules. Journal of Asset Management 15(4), 261-278.

Zhu, S., D. Li, and S. Wang (2009). Robust portfolio selection under downside risk measures. Quantitative Finance 9(7), 869-885.

Zweig, J. (1998). Five investing lessons from America's top pension fund. Money, January, $115-118$. 


\section{Ph.D. program in Economics \\ Declaration for admission to the doctoral examination}

I confirm

1. that the dissertation "Portfolio Strategies with Classical and Alternative Benchmarks" that I submitted was produced independently without assistance from external parties, and not contrary to high scientific standards and integrity,

2. that I have adhered to the examination regulations, including upholding a high degree of scientific integrity, which includes the strict and proper use of citations so that the inclusion of other ideas in the dissertation are clearly distinguished,

3. that in the process of completing this doctoral thesis, no intermediaries were compensated to assist me neither with the admissions or preparation processes, and in this process,

- no remuneration or equivalent compensation were provided

- no services were engaged that may contradict the purpose of producing a doctoral thesis

4. that I have not submitted this dissertation or parts of this dissertation elsewhere.

I am aware that false claims and the discovery of those false claims now, and in the future with regards to the declaration for admission to the doctoral examination can lead to the invalidation or revoking of the doctoral degree.

Place, Date

Laura-Chloé Kuntz 